\title{
GERAÇÃO DE AÇÃO DINÂMICA DE ESTRUTURAS BASEADA EM TRANSFORMADA DE WAVELET HARMÔNICA
}

\author{
Dissertação apresentada à Escola \\ Politécnica da Universidade de São Paulo \\ para a obtenção do Titulo de Mestre em \\ Engenharia
}

São Paulo 
PAULO SALVADOR BRITTO NIGRO

\section{GERAÇÃO DE AÇÃO DINÂMICA DE ESTRUTURAS BASEADA EM TRANSFORMADA DE WAVELET HARMÔNICA}

Dissertação apresentada à Escola

Politécnica da Universidade de São Paulo para a obtenção do Titulo de Mestre em Engenharia

Área de Concentração: Engenharia de Estruturas

Orientador: Prof. Dr. Reyolando Manoel Lopes Rebello da Fonseca Brasil

São Paulo 
Dedico este trabalho a todas as gerações de seres humanos que me possibilitaram herdar este conhecimento, espero tê-las honrado, e acrescentado algo a esta herança, para as próximas gerações que a herdarão. 


\section{AGRADECIMENTOS}

Ao Professor Doutor Reyolando M. L. R. F. Brasil pela escolha do tema, pela orientação e pela liberdade decisória que me foi concedida.

Aos meus pais pelo enorme apoio, ao meu tio avô Carlos Lyra por todo o suporte que me foi dado, ao afeto do meu avô Paulo Britto, e a dedicação e carinho da minha avó Maria Lúcia, que não chegou a ver este trabalho concluído.

Aos amigos que fiz no LMC pelo companherismo e ajuda acadêmica, aos Professores do PEF pelo conhecimento transmitido, e aos fucionários do PEF que sempre foram prestativos e atenciosos comigo.

A Escola Politécnica e a USP por toda infra-estrutura disponibilizada e por me fazerem ser parte de algo maior, e a cidade de São Paulo que me completou como cidadão.

A CAPES pelo repasse da bolsa de mestrado, e ao contribuinte o qual seus impostos permitiram que esta bolsa fosse possível, assim como toda a minha formação acadêmica, muito obrigado pela confiança em mim investida. 


\section{RESUMO}

Neste trabalho, é apresentado um modelo aperfeiçoado para gerar carregamentos dinâmicos pseudo-aleatórios para modelos estruturais sob excitação sísmica e de vento. Este é baseado no modelo de vento sintético proposto por Franco, diferindo pelo fato que usa a transformada de wavelet harmônica ao invés da série de Fourier, pois tem como objetivo descrever um comportamento não estacionário com a ajuda de uma função temporal. Para testar a qualidade do sinal desenvolvido neste trabalho, este foi comparado com sinais verdadeiro, das acelerações de sismos ocorrido na cidade de Hachinohe, no Japão e em El-centro, na Califórnia, e com um sinal gerado pelo modelo do sismo sintético de Corbani, este também baseado no modelo de vento sintético, com o uso de séries de Fourier. Em todas as análises feitas, foi mostrando que embora a geração de carregamentos com transformadas de wavelet harmônica seja mais complexa, esta possui um bom potencial para gerar carregamentos mais próximos da realidade do que métodos usuais baseados em carregamentos estacionários.

Palavras-chave: Wavelet harmônica, Carregamento dinâmico de estruturas, Vibrações aleatórias, Excitação sísmica e de vento, Comportamento não estacionário. 


\begin{abstract}
In this work is intruduced a improve model to create random loads to use in structural models under sismic and wind disturbance. The model is based on synthetic wind model intends by Franco, differing by the fact that applies harmonic wavelet transform instead of Fourier series, because it has the goal to describe a non stationary behavior with temporal function support. To test the quality from the signal developed in this work, it has been analyzed against true seismic acceleration signal that occurs from Hachinohe city in Japan and El-centro city in California, and with the synthetic seismic model developed by Corbani, that one descending on synthetic wind model, with Fourier series application. In all analysis, although loading creation with harmonic wavelet transform have been more sophisticated, that one has a great potencial to creat loading closer to the fact than usual methods based in stationary loading.
\end{abstract}

Keywords : Harmonic wavelet, Dynamic loading on structure, Random vibration, Seismic and wind excitation, Non stationary behavior. 


\section{LISTA DE FIGURAS}

Figura 2.1. Série temporal de comportamento aleatório (LAZANHA, 2003).....

Figura 2.2. Função de distribuição de probabilidade Normal (LAZANHA, 2003)

Figura 3.1. Aplicação da transformada janelada: (a) Sinal não estacionário; (b) Espectros de potência do sinal em determinados intervalos de tempo (Imagem modificada a partir de Barbosa; Blitzkow (2008)).

Figura 3.2. Espectro de amplitude: (a) com freqüências não-negativas; (b) com freqüências no domínio dos reais 32

Figura 3.3. Espectros de potência: (a) com freqüências não-negativas; (b) com frequiências positivas e negativas 33

Figura 3.4. Série discreta obtida através de uma série temporal contínua............................................ 36

Figura 4.1. Wavelet: (a) wavelet mãe; (b) wavelet filha com mudança de escala................................. 42

Figura 4.2. Wavelet: (a) wavelet mãe; (b) wavelet filha com mudança de translação............................. 42

Figura 4.3. Wavelet de Haar....................................................................................................... 48

Figura 4.4. Wavelet de Morlet: (a) parcela real; (b) parcela imaginária.............................................. 49

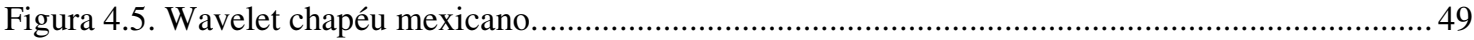

Figura 4.6. Parte da família de wavelets de Daubechies......................................................................52

Figura 4.7. Comparação entre as DFT: (a) Wavelet db20; (b) Wavelet harmônica; (c) DFT das duas, onde a db20 esta localizada a esquerda (Imagem modificada a partir de Newland (1993))..................54

Figura 4.8. Wavelet harmônica: (a) parcela real; (b) parcela imaginária.............................................. 55

Figura 4.9. Função de escala: (a) parcela real; (b) parcela imaginária. ................................................. 57

Figura 4.10. Espectro de freqüência obtido através de Transformadas de Fourier de wavelets harmônicas de diferentes níveis. .58

Figura 4.11. Espectro de frequiência obtido através de Transformadas de Fourier de wavelets harmônicas de diferentes níveis.

Figura 4.12. Janelas tempo-frequência: (a) Fourier janelada; (b) Wavelet (Imagem modificada a partir de Barbosa; Blitzkow (2008) )

Figura 4.13. Arrumação no escalograma dos coeficientes da wavelet harmônica: (a) com janela tempofreqüência de variação geométrica para q=2; (b) com janela tempo-frequiência de variação aritmética para $\mathrm{p}=2$.

Figura 4.14. Gráfico gerado com os dados da tabela 4.1 o qual relaciona a quantidade de coeficientes $\mathrm{N}$ (escala logarítmica) da DHWT em relação à quantidade de níveis n. ......................................... 80

Figura 5.1. Limites de integração da densidade espectral de potência para compor os harmônicos. .........84

Figura 5.2. Construção do espectograma: (a) Função de tendência ao quadrado e espectro de potência com comportamento estacionário; (b) A junção das duas funções gera o espectro de potência não estacionário.

90

Figura 5.3. Limites de integração da densidade espectral de potência para compor os coeficientes da transformada da wavelet harmônica. .92

Figura 5.4. Rajada equivalente determinada por Franco (1993). 100 
Figura 6.1. Numeração adotada para o modelo de shear building................................................... 110

Figura 6.2. Modelo de material elasto-plástico perfeito. ................................................................. 111

Figura 6.3. Função em relação ao tempo................................................................................. 116

Figura 6.4. Espectro de potência da função temporal......................................................................... 116

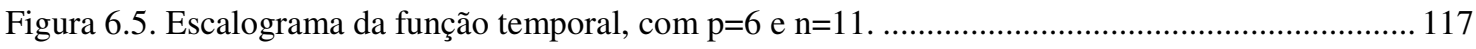

Figura 6.6. Forças devido ao vento na estrutura............................................................................ 119

Figura 6.7. São as curvas: (a) Espectro de freqüência; (b) Função de tendência.................................... 121

Figura 6.8. Pressão normalizada do vento atuante no centro de rajada, com comportamento localmente estacionário, gerado a partir de DHWT.

Figura 6.9. Pressão normalizada do vento atuante no centro de rajada, com comportamento próximo do estacionário, gerado a partir de Série de Fourier.

Figura 6.10. Escalograma da pressão normalizada do vento atuante no centro de rajada, com comportamento localmente estacionário, gerado a partir de DHWT.

Figura 6.11. Escalograma da pressão normalizada do vento atuante no centro de rajada, com comportamento próximo do estacionário, gerado a partir de Série de Fourier.

122

Figura 6.12. Espectro de potência da pressão normalizada do vento atuante no centro de rajada, com comportamento localmente estacionário, gerado a partir de DHWT.

Figura 6.13. Espectro de potência da pressão normalizada do vento atuante no centro de rajada, com comportamento próximo do estacionário, gerado a partir de Série de Fourier.

Figura 6.14. Deslocamentos do último pavimento devido a ações geradas por DHWT. 124

Figura 6.15. Deslocamentos do último pavimento devido a ações geradas por Série de Fourier. 124

Figura 6.16. São as curvas: (a) Espectro de freqüência; (b) Função de tendência................................. 128

Figura 6.17. Acelerograma do terremoto ocorrido na cidade de Hachinohe, em 1968 no Japão. ........... 129

Figura 6.18. Acelerograma com comportamento não estacionário, gerado pelo a partir de DHWT....... 129

Figura 6.19. Acelerograma com comportamento estacionário, gerado a partir de Série de Fourier........ 129

Figura 6.20. Escalograma do terremoto ocorrido na cidade de Hachinohe, em 1968 no Japão, baseado em seu acelerograma

Figura 6.21. Escalograma do acelerograma com comportamento não estacionário, gerado a partir de DHWT 130

Figura 6.22. Escalograma do acelerograma com comportamento aproximadamente estacionário, gerado a partir de Série de Fourier.

Figura 6.23. Espectro de potência do terremoto ocorrido na cidade de Hachinohe, em 1968 no Japão, baseado em seu acelerograma.

Figura 6.24. Espectro de potência do acelerograma com comportamento não estacionário, gerado a partir de DHWT.

Figura 6.25. Espectro de potência do acelerograma com comportamento aproximadamente estacionário, gerado a partir de Série de Fourier.

Figura 6.26. Deslocamentos do último pavimento devido a ações geradas por DHWT........................ 133

Figura 6.27. Deslocamentos do último pavimento devido a ações geradas por Série de Fourier............ 133

Figura 6.28. Resposta da estrutura devido ao acelerograma de Hachinohe........................................ 135

Figura 6.29. Resposta da estrutura devido à série 19, geradas a partir de DHWT. ............................... 135 
Figura 6.30. Resposta da estrutura devido à série 20, geradas a partir de SF. 135

Figura 6.31. São as curvas: (a) Espectro de freqüência; (b) Função de tendência 138

Figura 6.32. Acelerograma do terremoto ocorrido na cidade de El-centro, em 1940 na Califórnia. 139

Figura 6.33. Acelerograma com comportamento não estacionário, gerado pelo a partir de DHWT

Figura 6.34. Acelerograma com comportamento estacionário, gerado a partir de Série de Fourier.

Figura 6.35. Escalograma do terremoto ocorrido na cidade de El-centro, em 1940 na Califórnia, baseado em seu acelerograma. 140

Figura 6.36. Escalograma do acelerograma com comportamento não estacionário, gerado a partir de DHWT. 140

Figura 6.37. Escalograma do acelerograma com comportamento aproximadamente estacionário, gerado a partir de Série de Fourier.

Figura 6.38. Espectro de potência do terremoto ocorrido na cidade de El-centro, em 1940 na Califórnia, baseado em seu acelerograma 141

Figura 6.39. Espectro de potência do acelerograma com comportamento não estacionário, gerado a partir de DHWT. 141

Figura 6.40. Espectro de potência do acelerograma com comportamento estacionário, gerado a partir de Série de Fourier. 141

Figura 6.41. Deslocamentos do último pavimento devido a ações geradas por DHWT 143

Figura 6.42. Deslocamentos do último pavimento devido a ações geradas por Série de Fourier. 143

Figura 6.43. Resposta da estrutura devido ao acelerograma de El-Centro. 145

Figura 6.44. Resposta da estrutura devido à série 1, gerada a partir de DHWT 145

Figura 6.45. Resposta da estrutura devido à série 20, gerada a partir de SF. 


\section{LISTA DE TABELAS}

Tabela 4.1. Quantidade de coeficientes da DHWT em relação à quantidade de níveis para diferentes janelas tempo-freqüência.

Tabela 6.1. Dados estatísticos dos deslocamentos sofridos pela estrutura devido às séries dos dois modelos empregados.

Tabela 6.2. Comparação do Escalograma e do Espectro de potência dos dois métodos, com relação ao Escalograma e Espectro de Potência do sismo em Hachinohe.

Tabela 6.3. Dados estatísticos dos deslocamentos sofridos pela estrutura devido às séries dos dois modelos empregados, comparados ao deslocamento devido ao sismo real. 134

Tabela 6.4. Comparação do Escalograma e do Espectro de potência dos dois métodos, com relação ao Escalograma e Espectro de Potência do sismo em El-centro

Tabela 6.5. Dados estatísticos dos deslocamentos sofridos pela estrutura devido às séries dos dois modelos empregados, comparados ao deslocamento devido ao sismo real. 


\section{SUMÁRIO}

1. INTRODUÇÃ

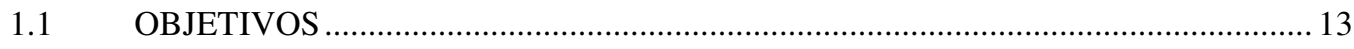

1.2 PLANO DE DISSERTAÇÃO ............................................................................... 15

2. PARÂMETROS ESTATÍSTICOS E PROBABILÍSTICOS .............................17

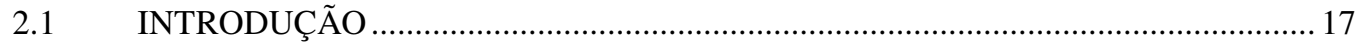

2.2 FUNÇÃO DENSIDADE DE PROBABILIDADE...................................................... 17

2.3 DISTRIBUIÇÃO GAUSSIANA OU NORMAL ........................................................... 18

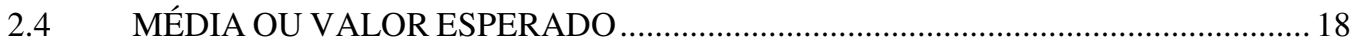

2.5 VALOR QUADRADO MÉDIO OU POTÊNCIA MÉDIA ………................................ 19

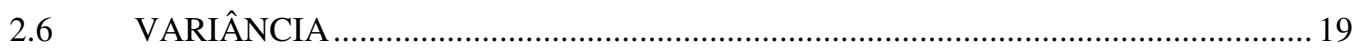

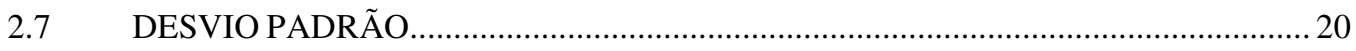

2.8 FUNÇÃO DISTRIBUIÇÃO DE PROBABILIDADE .................................................. 20

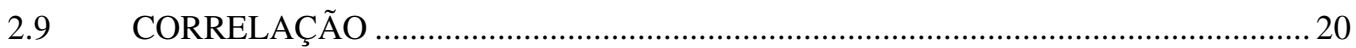

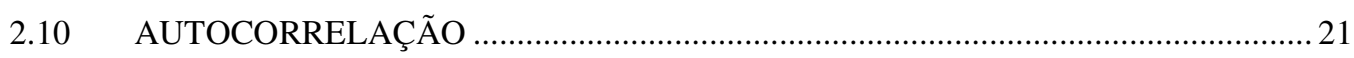

2.11 COVARIÂNCIA OU COEFICIENTE DE CORRELAÇÃO........................................... 22

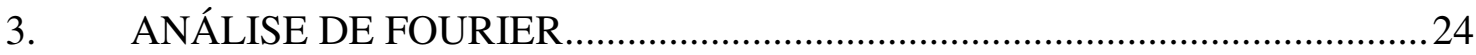

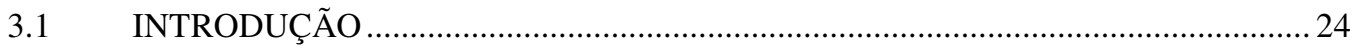

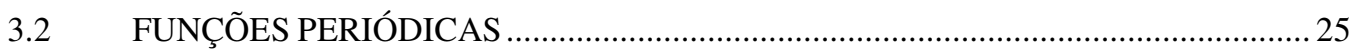

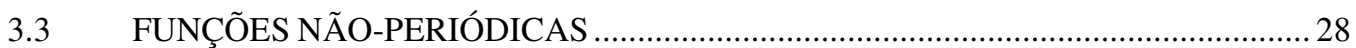

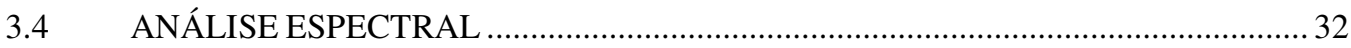

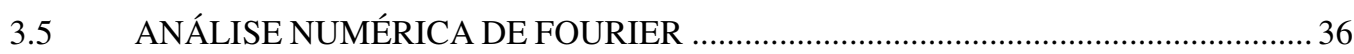

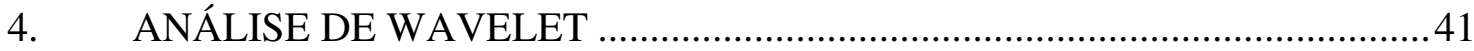

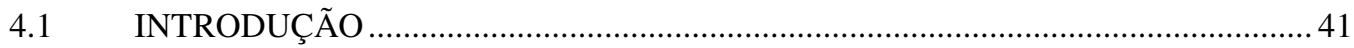

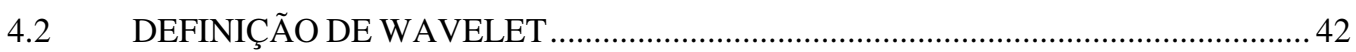

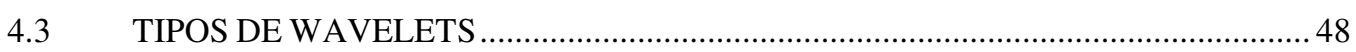

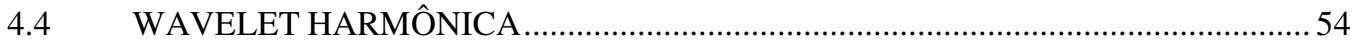

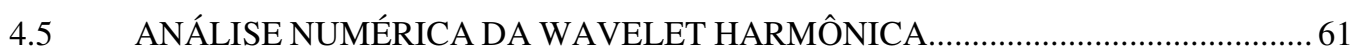

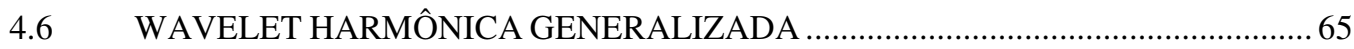

4.7 ANÁLISE NUMÉRICA DA WAVELET HARMÔNICA GENERALIZADA............. 71

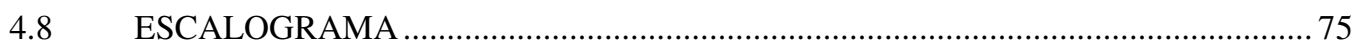


5. $\quad$ ANÁLISE DE AÇÕES ALEATÓRIAS …................................................... 82

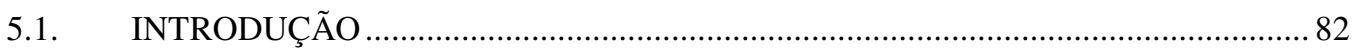

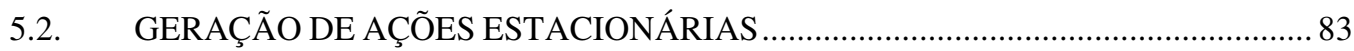

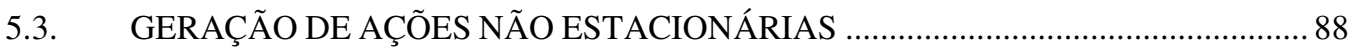

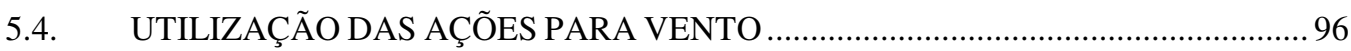

5.5. UTILIZAÇÃO DAS AÇÕES PARA SISMO …………………………………..... 104

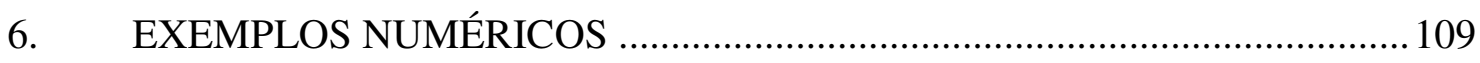

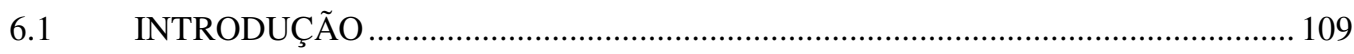

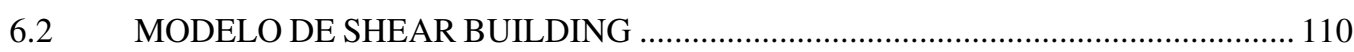

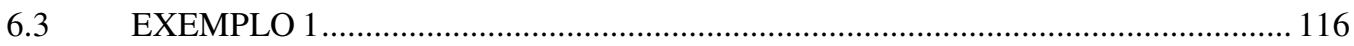

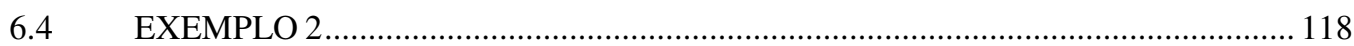

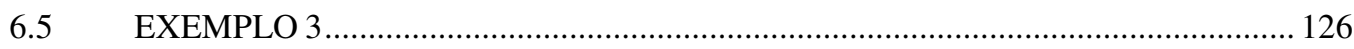

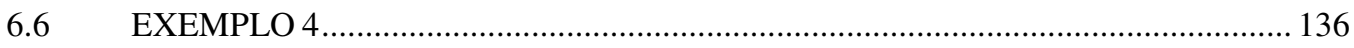

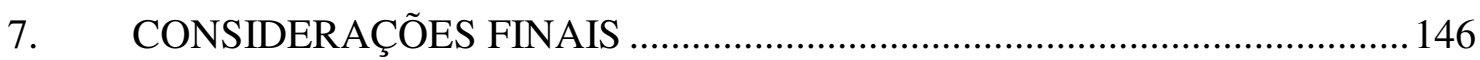

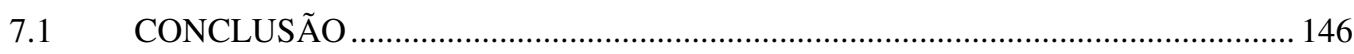

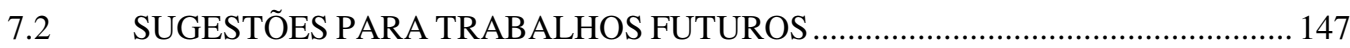

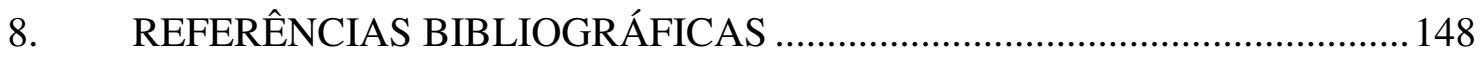




\section{INTRODUÇÃO}

\subsection{OBJETIVOS}

As wavelets são funções de comportamento oscilatório, média nula e energia finita, sendo bastante útil na representação de processos estocásticos com comportamento nãoestacionário, como na geração de ações para estruturas, sejam eles devido ao vento ou a sismos, onde geralmente estes são representados por comportamentos estacionários, que não possuem controle de suas características temporais.

Entende-se por ação tudo aquilo que causa uma exitação na estrutura, seja através de um carregamento ou acelerações ocorridas no solo, devido a sismos. E entende-se por comportamento não estacionário todo aquele que não mantêm suas propriedades como amplitudes sem tendências e permanência do mesmo intervalo de freqüências ao longo do tempo, resultando em parâmetros estatísticos e espectros de frequiência distintos para diferentes intervalos de tempo analisados.

Na realidade, praticamente todo o sinal é não estacionário, pelo simples fato que este é finito, resultando em uma grande variação de valores em seu começo e final. Quando se analisa sismos é bastante notório que em um intervalo geralmente menor que um minuto, as acelerações inicialmente com grandes amplitude decaiam rapidamente para próximo de zero.

Até mesmo o vento, quase sempre considerado estacionário para fins práticos, quando são analisados seus sinais em espectros com domínio do tempo e da freqüência, notamse pequenas variações não-periódicas de amplitude, confirmando assim sua não estacionaridade.

A wavelet ou ondaleta, como sugerida por Morettin (1999), é uma função capaz de descrever e decompor funções no domínio da frequiência de forma a ser possível a análise em diferentes escalas de frequiência e de tempo. Uma das grandes diferenças da análise de wavelet em relação a análise de Fourier é que na primeira há uma concentração de energia em uma região finita, já que a ultima usa as funções de seno e cosseno que são periódicas. 
Outra diferença é que na análise de Fourier pode-se extrair com bastante precisão informações sobre o domínio da freqüência e razoável precisão no tempo com o uso do conceito de janela espectral, onde este trabalha com uma janela tempo-frequiência fixa, o que torna difícil a captura de altas e baixas frequencia de um sinal simultaneamente, sendo este problema resolvido pela análise de wavelet.

Neste trabalho, que usa como idéia inicial o método do vento sintético, desenvolvido por Franco (1993), tem como objetivo o uso de uma wavelet do tipo harmônica onde sua transformada com ajuda de funções pseudo-aleatórias, gera carregamentos não estacionários baseados em tendências e intervalos de frequiência pré-determinados, sendo este comparado com carregamentos reais e modelos de carregamento baseado no método do vento sintético.

O uso da análise de wavelet, principalmente para a geração de carregamentos em estruturas é um campo novo e importante de pesquisa, possibilitando uma análise mais detalhada, pois embora existam métodos numéricos cada vez mais precisos de análise estrutural, esta análise pode ter uma precisão inferior, caso o dado de entrada, ou seja, o carregamento, não seja bem controlado e definido. 


\subsection{PLANO DE DISSERTAÇÃO}

Com o intuito de facilitar a pesquisa neste trabalho, o resumo do conteúdo de cada capítulo é apresentado:

O capítulo 2 tem como objetivo a fundamentação dos parâmetros estatísticos mais usuais apresentando a função de distribuição de probabilidade Gaussiana e seus parâmetros como a média e variância, são também explicitados os conceitos de correlação e sua variação, a autocorrelação, e termina por mostrar o conceito de covariância.

O capítulo 3 faz uma revisão bibliográfica da análise de Fourier, mostrando a representação de funções periódicas através da série de Fourier, e a representação de funções não periódicas através da transformada de Fourier, em seguida é apresentada à análise espectral, contendo o conceito de densidade espectral de potência e transformada de Fourier com janela espectral. Por ultimo é abordada a análise numérica, com a dedução da DFT e uma breve explicação da FFT.

O capítulo 4 faz uma revisão bibliográfica da análise de wavelet, explicando os critérios necessários para uma função ser classificada como wavelet, além da diferença entre transformadas contínuas e discretas de wavelet, também são mostradas algumas wavelets, com uma breve explicação sobre a wavelet de Daubechies. Por ultimo é mostrado o desenvolvimento da wavelet harmônica e sua análise numérica, assim como o conceito e desenvolvimento do escalograma para este tipo de wavelet.

O capitulo 5 contém a parte principal deste trabalho, mostrando a geração de carregamentos com a aplicação de funções pseudo-aleatórias para comportamento estacionário e não estacionário, neste ultimo, utilizando a transformada da wavelet harmônica obtida através de escalogramas, onde estes são gerados pela combinação de densidades espectrais de potência e funções para a representação de tendências, alem de aplicações deste carregamentos para vento e sismo. 
O capítulo 6 mostra exemplos de possíveis aplicações, como estrutura sujeita a pressões devido ao vento e acelerações de sismos, onde são comparadas as respostas elásticas e elasto-plásticas do modelo de comportamento não estacionário descrito neste trabalho com sismos reais e o modelo do sismo sintético, este último de comportamento estacionário, o qual utiliza valores pseudo-aleatórios aplicados em uma série de Fourier.

No capitulo 7 são mostradas as conclusões do que foi pesquisado, e propostas para trabalhos futuros, seguidas do capítulo 8, este, de referências bibliográficas. 


\section{PARÂMETROS ESTATÍSTICOS E PROBABILÍSTICOS}

\subsection{INTRODUÇÃO}

O estudo de probabilidade fornece a base para o estudo da teoria de vibrações aleatórias, e de acordo com Blessmann (1998), dados para os quais não é possível prever um valor exato em certo instante, com variação aleatória, e indefinição de relações matemáticas explicita, são analisados pela teoria da probabilidade e médias estatísticas. A seguir, serão expostos alguns conceitos fundamentais da teoria da probabilidade e algumas médias estatísticas.

\subsection{FUNÇÃO DENSIDADE DE PROBABILIDADE}

Considere $\mathrm{x}(\mathrm{t})$, que representa uma série temporal, de comportamento aleatório (Figura 2.1), onde se deseja saber a probabilidade na qual $\mathrm{x} \leq \mathrm{x}(\mathrm{t}) \leq \mathrm{x}+\mathrm{dx}$ ocorra em um intervalo de tempo T longo o suficiente.

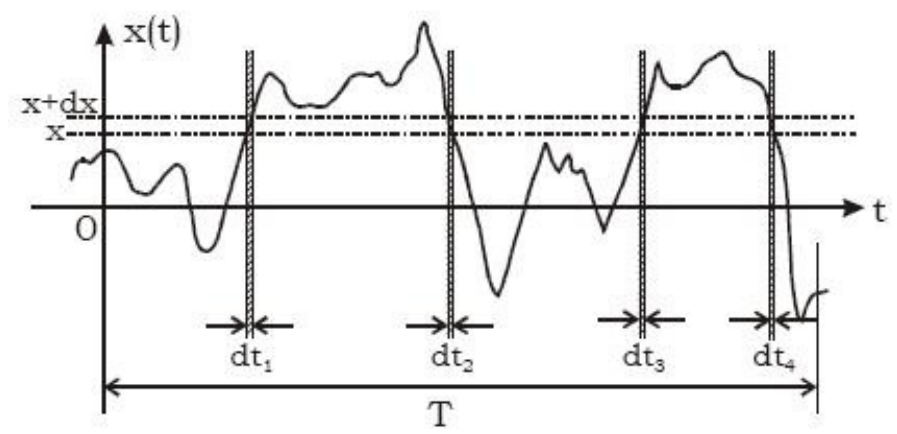

Figura 2.1. Série temporal de comportamento aleatório (LAZANHA, 2003)

Para isso, intuitivamente, pode-se fazer:

$\frac{\sum \mathrm{dt}}{\mathrm{T}}=\frac{\mathrm{dt}_{1}+\mathrm{dt}_{2}+\mathrm{dt}_{3}+\mathrm{dt}_{4}}{\mathrm{~T}}$

Onde a equação (2.1) representa a fração ou probabilidade de ocorrência no tempo T do intervalo entre $\mathrm{x}$ e $\mathrm{x}+\mathrm{dx}$. Pode-se obter a mesma fração de uma forma menos trabalhosa, através da equação (2.2): 
$\frac{\sum \mathrm{dt}}{\mathrm{T}}=p(\mathrm{x})(\mathrm{x}+\mathrm{dx}-\mathrm{x})=p(\mathrm{x}) \mathrm{dx}$

Dentro da equação (2.2), a função $p(\mathrm{x})$ representa a relação ou densidade para a distribuição dos valores de $\mathrm{x}$ de forma que se possa obter a mesma fração ou probabilidade utilizando diretamente o intervalo entre $\mathrm{x}$ e $\mathrm{x}+\mathrm{dx}$. Para a função $p(\mathrm{x})$ usase o nome de função densidade de probabilidade.

\subsection{DISTRIBUIÇÃO GAUSSIANA OU NORMAL}

Segundo Newland (1993), muitas vibrações aleatórias possuem uma função de distribuição de probabilidade em formato de sino (Figura 2.2), chamada de Gaussiana ou Normal.

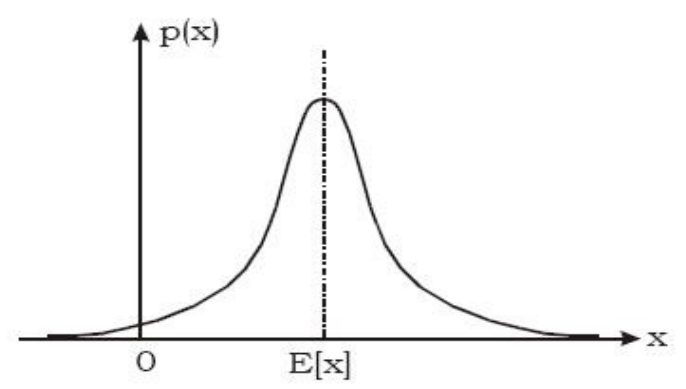

Figura 2.2. Função de distribuição de probabilidade Normal (LAZANHA, 2003)

O formato de sino é gerado pela equação (2.3):

$p(\mathrm{x})=\frac{1}{\sqrt{2 \pi} \sigma} \mathrm{e}^{\frac{-(\mathrm{x}-\mathrm{E}[\mathrm{x}])^{2}}{2 \sigma^{2}}}$

Aonde são utilizados a média e a variância como parâmetros estatísticos.

\subsection{MÉDIA OU VALOR ESPERADO}

$\mathrm{O}$ valor gerado pela área sob a curva $\mathrm{x}(\mathrm{t})$ em um intervalo de tempo $\mathrm{T}$, dividida pelo intervalo de tempo $\mathrm{T}$.

$$
E[x(t)]=\int_{-T / 2}^{+T / 2} x(t) \frac{d t}{T}
$$


Convertendo a integral em somatório:

$E\left[x\left(t_{i}\right)\right]=\sum_{i} x\left(t_{i}\right) \frac{d t_{i}}{T}$

Substituindo a (2.2) em (2.5), e convertendo somatório em integral:

$\mathrm{E}[\mathrm{x}]=\int_{-\infty}^{+\infty} \mathrm{x} p(\mathrm{x}) \mathrm{dx}$

Onde a equação (2.6) representa a definição principal da média.

\subsection{VALOR QUADRADO MÉDIO OU POTÊNCIA MÉDIA}

A mesma definição da média, diferindo apenas no uso do quadrado da curva x(t).

$E\left[x(t)^{2}\right]=\int_{-T / 2}^{+T / 2} x(t)^{2} \frac{d t}{T}$

Utilizando a mesma resolução para a equação (2.6), chega-se:

$\mathrm{E}\left[\mathrm{x}^{2}\right]=\int_{-\infty}^{+\infty} \mathrm{x}^{2} p(\mathrm{x}) \mathrm{dx}$

Onde (2.8) representa a definição principal do valor quadrado médio.

\subsection{VARIÂNCIA}

$\mathrm{O}$ valor médio do quadrado da variação dos valores da curva $\mathrm{x}(\mathrm{t})$ em relação ao valor médio de $\mathrm{x}(\mathrm{t})$.

$$
\sigma_{\mathrm{x}}^{2}=\mathrm{E}\left[(\mathrm{x}-\mathrm{E}[\mathrm{x}])^{2}\right]
$$

Desenvolvendo o quadrado da soma e considerando o fato que a média do todo é igual a média de cada parcela, pela equação (2.9) chega-se:

$$
\sigma_{\mathrm{x}}^{2}=\mathrm{E}\left[\mathrm{x}^{2}\right]-(\mathrm{E}[\mathrm{x}])^{2}
$$

Onde a (2.10) representa a definição principal da variância. 


\subsection{DESVIO PADRÃO}

É definido como a raiz quadrada da variância.

$\sigma_{\mathrm{x}}=\sqrt{\mathrm{E}\left[\mathrm{x}^{2}\right]-(\mathrm{E}[\mathrm{x}])^{2}}$

\subsection{FUNÇÃO DISTRIBUIÇÃO DE PROBABILIDADE}

Definida como a área sob a função densidade de probabilidade, segundo Newland (1993), descreve a distribuição de valores de uma variável aleatória.

$$
\mathrm{P}(\mathrm{x})=\int_{-\infty}^{\mathrm{x}} p(x) \mathrm{d} x \quad 0 \leq \mathrm{P}(\mathrm{x}) \leq 1
$$

$\mathrm{O}$ valor da equação (2.12) varia entre $0 \leq \mathrm{P}(\mathrm{x}) \leq 1$, para um valor de $\mathrm{x}=\mathrm{a}$, tem-se:

$$
\begin{aligned}
& \operatorname{Prob}(\mathrm{x} \leq \mathrm{a})=\int_{-\infty}^{\mathrm{a}} p(\mathrm{x}) \mathrm{dx}=\mathrm{P}(\mathrm{a}) \\
& \operatorname{Prob}(\mathrm{x} \geq \mathrm{a})=\int_{\mathrm{a}}^{\infty} p(\mathrm{x}) \mathrm{dx}=1-\mathrm{P}(\mathrm{a})
\end{aligned}
$$

Para o intervalo que cubra todo o domínio de x, tem-se:

$$
\operatorname{Prob}(-\infty \leq x \leq \infty)=\int_{-\infty}^{\infty} p(\mathrm{x}) \mathrm{dx}=1
$$

Que equivale à certeza da ocorrência.

\subsection{CORRELAÇÃO}

Fornecidas duas séries temporais $\mathrm{x}(\mathrm{t})$ e $\mathrm{y}(\mathrm{t})$, uma maneira de expressar a relação entre estas séries é através do conceito de correlação, esse importante conceito é utilizado para determinar os coeficientes de séries de funções trigonométricas ou de ondaletas, com comportamento estacionário ou não-estacionário, sendo um método extremamente importante para a análise de sinais. Através da definição de quadrado médio, mas utilizando o produto de duas curvas distintas ao invés do produto da mesma e partindo da equação (2.7), tem-se: 
$E[x(t) y(t)]=\int_{-T / 2}^{+T / 2} x(t) y(t) \frac{d t}{T}$

Ou, deslocando o intervalo de integração:

$E[x(t) y(t)]=\int_{0}^{T} x(t) y(t) \frac{d t}{T}$

\subsection{AUTOCORRELAÇÃO}

Considerando agora uma única série temporal, o objetivo é analisar sua variação ao longo do tempo, para isto é imposta uma defasagem de tempo $\tau$, e uma maneira de expressar a relação desta defasagem de tempo é chamada de autocorrelação. Através da definição de quadrado médio, mas utilizando o produto de duas curvas iguais defasadas no tempo em $\tau$ e partindo da equação (2.7), tem-se

$$
E[x(t) x(t+\tau)]=\int_{-T / 2}^{+T / 2} x(t) x(t+\tau) \frac{d t}{T}
$$

Ou, deslocando o domínio da integral:

$$
E[x(t) x(t+\tau)]=\int_{0}^{T} x(t) x(t+\tau) \frac{d t}{T}
$$

Quando $\tau=0$, a autocorrelação adquire o valor do quadrado médio:

$$
E[x(t) x(t+0)]=E\left[x^{2}(t)\right]
$$

Para um comportamento estacionário, o valor da autocorrelação é independente do valor absoluto do tempo, então a equação (2.18) é chamada de função de autocorrelação, esta pode ser expressa como:

$\mathrm{R}_{\mathrm{x}}(\tau)=\mathrm{E}[\mathrm{x}(\mathrm{t}) \mathrm{x}(\mathrm{t}+\tau)]$

Novamente devido ao comportamento estacionário, pode-se observar o comportamento par da função de autocorrelação: 
$\mathrm{R}_{\mathrm{x}}(\tau)=\mathrm{E}[\mathrm{x}(\mathrm{t}) \mathrm{x}(\mathrm{t}+\tau)]=\mathrm{E}[\mathrm{x}(\mathrm{t}) \mathrm{x}(\mathrm{t}-\tau)]=\mathrm{R}_{\mathrm{x}}(-\tau)$

\subsection{COVARIÂNCIA OU COEFICIENTE DE CORRELAÇÃO}

Fornecidas duas séries temporais $\mathrm{x}(\mathrm{t})$ e $\mathrm{y}(\mathrm{t})$, e supondo que cada par de valores é representado por um ponto em um gráfico onde cada série temporal representa um eixo, quando os valores destas séries forem bastante distintos, formarão uma nuvem de pontos amorfa, por outro lado, quando os valores destas séries forem similares, esta nuvem de pontos irá gerar uma reta de regressão com coeficiente angular próximo da unidade. O coeficiente angular desta reta de regressão é chamado de covariância ou coeficiente de correlação e corresponde a equação (2.23):

$$
\rho_{\mathrm{xy}}=\frac{\mathrm{E}[(\mathrm{x}-\mathrm{E}[\mathrm{x}])] \mathrm{E}[(\mathrm{y}-\mathrm{E}[\mathrm{y}])]}{\sigma_{\mathrm{x}} \sigma_{\mathrm{y}}} \quad-1 \leq \rho_{\mathrm{xy}} \leq 1
$$

Para $\rho_{\mathrm{xy}}=1$, é equivalente a $\mathrm{y}=\mathrm{x}$, para $\rho_{\mathrm{xy}}=0$, não há correlação, e para o valor de $\rho_{\mathrm{xy}}=-1$ quando for equivalente a $\mathrm{y}=-\mathrm{x}$. A partir de (2.23), desenvolvendo o quadrado da soma e considerando o fato que a média do todo é igual a média de cada parcela, chega-se:

$$
\rho_{\mathrm{xy}}=\frac{\mathrm{E}[\mathrm{x}(\mathrm{t}) \mathrm{y}(\mathrm{t})]-\mathrm{E}[\mathrm{x}(\mathrm{t})] \mathrm{E}[\mathrm{y}(\mathrm{t})]}{\sigma_{\mathrm{x}} \sigma_{\mathrm{y}}} \quad-1 \leq \rho_{\mathrm{xy}} \leq 1
$$

Quando se utiliza uma única série temporal, com o intuito de analisar sua variação ao longo do tempo, definimos o coeficiente de autocorrelação, que corresponde a equação (2.25):

$$
\rho=\frac{\mathrm{E}[\mathrm{x}(\mathrm{t}) \mathrm{x}(\mathrm{t}+\tau)]-\mathrm{E}[\mathrm{x}(\mathrm{t})] \mathrm{E}[\mathrm{x}(\mathrm{t}+\tau)]}{\sigma_{\mathrm{x}(\mathrm{t})} \sigma_{\mathrm{x}(\mathrm{t}+\tau)}} \quad-1 \leq \rho \leq 1
$$

Aplicando a equação (2.21) a equação (2.25), chega-se:

$$
\rho=\frac{\mathrm{R}_{\mathrm{x}}(\tau)-(\mathrm{E}[\mathrm{x}(\mathrm{t})])^{2}}{\sigma_{\mathrm{x}(\mathrm{t})}^{2}} \quad-1 \leq \rho \leq 1
$$


Com a equação (2.26), a função de autocorrelação pode ser expressa por:

$\mathrm{R}_{\mathrm{x}}(\tau)=\rho \sigma_{\mathrm{x}(\mathrm{t})}^{2}+(\mathrm{E}[\mathrm{x}(\mathrm{t})])^{2} \quad-1 \leq \rho \leq 1$

E devido ao coeficiente de autocorrelação, a função de autocorrelação tem os valores limitados por:

$-\sigma_{\mathrm{x}(\mathrm{t})}^{2}+(\mathrm{E}[\mathrm{x}(\mathrm{t})])^{2} \leq \mathrm{R}_{\mathrm{x}}(\tau) \leq \sigma_{\mathrm{x}(\mathrm{t})}^{2}+(\mathrm{E}[\mathrm{x}(\mathrm{t})])^{2}$

Quando $\tau=0$, resulta em $\rho=1$, então:

$\mathrm{R}_{\mathrm{x}}(0)=\sigma_{\mathrm{x}(\mathrm{t})}^{2}+(\mathrm{E}[\mathrm{x}(\mathrm{t})])^{2}=\mathrm{E}\left[\mathrm{x}(\mathrm{t})^{2}\right]$

Quando $\tau \rightarrow \infty$, resulta em $\rho=0$, então:

$\mathrm{R}_{\mathrm{x}}(\tau \rightarrow \infty)=(\mathrm{E}[\mathrm{x}(\mathrm{t})])^{2}$ 


\section{ANÁLISE DE FOURIER}

\subsection{INTRODUÇÃO}

Este é um dos mais antigos assuntos de análise matemática, que começou a ser desenvolvido pelo físico-matemático Jean-Baptiste Joseph Fourier, sendo estudado por grandes físico-matemáticos como Euler, d'Alembert e Lagrange, pois possui uma importância enorme para a matemática, física e engenharia. Analisando a prática, quando se pensa em análise de Fourier, geralmente se refere à série de Fourier e a transformada de Fourier.

A relevância da série de Fourier e da Transformada de Fourier não vem apenas da interpretação de seu significado físico, como análise tempo-freqüência de sinais, mas pelo fato que as técnicas de análise de Fourier serem extremamente poderosas, como no estudo de análise de wavelets, em especial para as wavelets harmônicas, que são a ferramenta matemática principal deste trabalho. 


\subsection{FUNÇÕES PERIÓDICAS}

Parte do princípio que uma função periódica $\mathrm{x}(\mathrm{t})$, com período $\mathrm{T}$, a qual pode ser correlacionada com funções periódicas e harmônicas, sendo a função periódica sintetizada por uma série destas funções. Segundo Chierice (2007), esta série pode ser também entendida como uma combinação linear de senos e cossenos, cujas freqüências são múltiplos inteiros de uma freqüência básica. Essa combinação linear é então denominada série de Fourier. É possível mostrar essa série em uma notação compactada:

$\mathrm{x}(\mathrm{t})=a_{0}+2 \sum_{\mathrm{k}=1}^{+\infty}\left[a_{\mathrm{k}} \cos \left(\omega_{\mathrm{k}} \mathrm{t}\right)+b_{\mathrm{k}} \operatorname{sen}\left(\omega_{\mathrm{k}} \mathrm{t}\right)\right]$

Onde:

$a_{0}=\frac{1}{\mathrm{~T}} \int_{-\mathrm{T} / 2}^{+\mathrm{T} / 2} \mathrm{x}(\mathrm{t}) \mathrm{dt}$

$a_{\mathrm{k}}=\frac{1}{\mathrm{~T}} \int_{-\mathrm{T} / 2}^{+\mathrm{T} / 2} \mathrm{x}(\mathrm{t}) \cos \left(\omega_{\mathrm{k}} \mathrm{t}\right) \mathrm{dt}$

$b_{\mathrm{k}}=\frac{1}{\mathrm{~T}} \int_{-\mathrm{T} / 2}^{+\mathrm{T} / 2} \mathrm{x}(\mathrm{t}) \operatorname{sen}\left(\omega_{\mathrm{k}} \mathrm{t}\right) \mathrm{dt}$

Ou como é usualmente apresentada, com o 2 incluso em seus coeficientes:

$\mathrm{x}(\mathrm{t})=a_{0}+\sum_{\mathrm{k}=1}^{+\infty}\left[a_{\mathrm{k}} \cos \left(\omega_{\mathrm{k}} \mathrm{t}\right)+b_{\mathrm{k}} \operatorname{sen}\left(\omega_{\mathrm{k}} \mathrm{t}\right)\right]$

Onde:

$$
\begin{aligned}
& a_{\mathrm{k}}=\frac{2}{\mathrm{~T}} \int_{-\mathrm{T} / 2}^{+\mathrm{T} / 2} \mathrm{x}(\mathrm{t}) \cos \left(\omega_{\mathrm{k}} \mathrm{t}\right) \mathrm{dt} \\
& b_{\mathrm{k}}=\frac{2}{\mathrm{~T}} \int_{-\mathrm{T} / 2}^{+\mathrm{T} / 2} \mathrm{x}(\mathrm{t}) \operatorname{sen}\left(\omega_{\mathrm{k}} \mathrm{t}\right) \mathrm{dt}
\end{aligned}
$$

Sendo $\omega_{\mathrm{k}}$ os valores das freqüências angulares, definidos por: 
$\omega_{\mathrm{k}}=\frac{2 \pi \mathrm{k}}{\mathrm{T}}$

Com um espaçamento entre freqüências angulares adjacentes de:

$\Delta \omega=\omega_{\mathrm{k}+1}-\omega_{\mathrm{k}}=\frac{2 \pi(\mathrm{k}+1)}{\mathrm{T}}-\frac{2 \pi \mathrm{k}}{\mathrm{T}}=\frac{2 \pi}{\mathrm{T}}$

Como citado anteriormente, os coeficientes dessa série representam a correlação entre a função periódica $\mathrm{x}(\mathrm{t})$ e as funções cosseno para $a_{\mathrm{k}}$ (3.6) e seno para $b_{\mathrm{k}}$ (3.7), a multiplicação por 2 é utilizada para evitar o uso de freqüências angulares negativas. Quando $\mathrm{k}=0, \cos (0)=1$, gerando $a_{0}(3.2)$, como $\operatorname{sen}(0)=0$ resulta em $b_{0}=0$.

Newland (1993) explica que uma importante restrição da série de Fourier acontece quando $\mathrm{x}(\mathrm{t})$ possui descontinuidades, onde a série irá gerar um valor médio no local. Há outra maneira de representar a série de Fourier, sendo esta representação amplamente utilizada para processos estocásticos, onde a série pode ser obtida considerando os pares de coeficientes $a_{\mathrm{k}}$ e $b_{\mathrm{k}}$ como coordenadas de um vetor em um ângulo $\phi_{\mathrm{k}}$ com a horizontal, e as funções seno e cosseno como coordenadas de outro vetor com um ângulo $\omega_{\mathrm{k}} \mathrm{t}$ com a horizontal, utilizando a definição de produto escalar entre estes vetores, chega-se:

$\mathrm{x}(\mathrm{t})=C_{0}+\sum_{\mathrm{k}=1}^{\infty} C_{\mathrm{k}} \cos \left(\omega_{\mathrm{k}} \mathrm{t}-\phi_{\mathrm{k}}\right)$

Onde:

$C_{0}=a_{0}$

$C_{\mathrm{k}}=\sqrt{a_{\mathrm{k}}^{2}+b_{\mathrm{k}}^{2}}$

$\phi_{\mathrm{k}}=\frac{b_{\mathrm{k}}}{a_{\mathrm{k}}}$

A série de Fourier também pode ser representada na forma complexa, permitindo um modo mais compacto de representação. Para que isto ocorra, é necessário fazer uso das relações: 
$\operatorname{sen}\left(\omega_{\mathrm{k}} \mathrm{t}\right)=\frac{i\left(\mathrm{e}^{-i \omega_{\mathrm{k}} \mathrm{t}}-\mathrm{e}^{i \omega_{\mathrm{k}} \mathrm{t}}\right)}{2}$

$\cos \left(\omega_{\mathrm{k}} \mathrm{t}\right)=\frac{\left(\mathrm{e}^{-i \omega_{\mathrm{k}} \mathrm{t}}+\mathrm{e}^{i \omega_{\mathrm{k}} \mathrm{t}}\right)}{2}$

Substituindo as relações (3.14) e (3.15) na equação (3.5), tem-se:

$\mathrm{x}(\mathrm{t})=D_{0}+\sum_{\mathrm{k}=1}^{+\infty}\left[D_{\mathrm{k}} \mathrm{e}^{\mathrm{i} \omega_{\mathrm{k}} \mathrm{t}}+\bar{D}_{\mathrm{k}} \mathrm{e}^{-\mathrm{i} \omega_{\mathrm{k}} \mathrm{t}}\right]$

Onde:

$D_{0}=a_{0}$

$D_{\mathrm{k}}=\frac{a_{\mathrm{k}}-\mathrm{i} b_{\mathrm{k}}}{2}$

$\bar{D}_{\mathrm{k}}=\frac{a_{\mathrm{k}}+\mathrm{i} b_{\mathrm{k}}}{2}$

Sendo (3.19) o conjugado complexo de (3.18). Para simplificar a equação (3.16), podese introduzir, apenas por uma questão matemática, o conceito de freqüência negativa:

$\mathrm{x}(\mathrm{t})=\sum_{\mathrm{k}=-\infty}^{+\infty}\left[D_{\mathrm{k}} \mathrm{e}^{\mathrm{i} \omega_{\mathrm{k}} \mathrm{t}}\right]$

Devido a todas estas representações, podem-se obter as seguintes relações:

$a_{0}=C_{0}=D_{0}$

$C_{\mathrm{k}}=2\left|D_{\mathrm{k}}\right|=2\left|\bar{D}_{\mathrm{k}}\right| \quad$ para $\quad \mathrm{k} \geq 1$

Os coeficientes da série de Fourier podem ter uma representação gráfica, onde o eixo das abscissas representa os valores das freqüências angulares, e os eixos das ordenadas representam os coeficientes de cada tipo de função da série de Fourier. 


\subsection{FUNÇÕES NÃO-PERIÓDICAS}

De acordo com Newland (1993), quando o período T, da função x(t) vai se tornando extenso, o espaçamento entre as freqüências angulares adjacentes vai se tornando pequeno, assim como a diferença entre os valores dos coeficientes de Fourier adjacentes. Quando o período T tende ao infinito, o comportamento não pode mais ser analisado como componentes de freqüência angular e coeficientes de Fourier discretos, a série de Fourier se converte em uma integral de Fourier e os coeficientes de Fourier se convertem em funções contínuas da freqüência chamada de Transformadas de Fourier. Então, estas mudanças começam com a manipulação da equação (3.9), tal que:

$\frac{\Delta \omega}{\pi}=\frac{2}{\mathrm{~T}}$

Substituindo a relação dada por (3.23), em (3.6) e (3.7), em seguida levando para (3.5), tem-se:

$x(t)=\sum_{k=1}^{+\infty}\left[\begin{array}{l}\left(\frac{\Delta \omega}{\pi} \int_{-T / 2}^{+T / 2} x(t) \cos \left(\omega_{k} t\right) d t\right) \cos \left(\omega_{k} t\right)+ \\ +\left(\frac{\Delta \omega}{\pi} \int_{-T / 2}^{+T / 2} x(t) \operatorname{sen}\left(\omega_{k} t\right) d t\right) \operatorname{sen}\left(\omega_{k} t\right)\end{array}\right]$

Quando $\mathrm{T} \rightarrow \infty, \Delta \omega \rightarrow \mathrm{d} \omega$ e substituindo o somatório pela integral com os limites entre $0 \leq \omega \leq \infty$, o que inclui o coeficiente $a_{0}$, chega-se:

$x(t)=\int_{0}^{+\infty}\left[\begin{array}{l}\left(\frac{1}{\pi} \int_{-\infty}^{+\infty} x(t) \cos (\omega t) d t\right) \cos (\omega t)+ \\ +\left(\frac{1}{\pi} \int_{-\infty}^{+\infty} x(t) \operatorname{sen}(\omega t) d t\right) \operatorname{sen}(\omega t)\end{array}\right] d \omega$

Onde, por definição:

$$
\begin{aligned}
& A(\omega)=\frac{1}{2 \pi} \int_{-\infty}^{+\infty} x(t) \cos (\omega t) d t \\
& B(\omega)=\frac{1}{2 \pi} \int_{-\infty}^{+\infty} x(t) \operatorname{sen}(\omega t) d t
\end{aligned}
$$

Resultando em: 
$x(t)=2 \int_{0}^{+\infty} A(\omega) \cos (\omega t) d \omega+2 \int_{0}^{+\infty} B(\omega) \operatorname{sen}(\omega t) d \omega$

Que pode ser escrita de forma equivalente:

$\mathrm{x}(\mathrm{t})=\int_{-\infty}^{+\infty} \mathrm{A}(\omega) \cos (\omega \mathrm{t}) \mathrm{d} \omega+\int_{-\infty}^{+\infty} \mathrm{B}(\omega) \operatorname{sen}(\omega \mathrm{t}) \mathrm{d} \omega$

As equações (3.26) e (3.27) são os componentes da Transformadas de Fourier de x(t), analisando estas equações, percebe-se que (3.26) é uma função par, onde $\mathrm{A}(-\omega)=\mathrm{A}(\omega)$ e (3.27) é uma função impar, pois $\mathrm{B}(-\omega)=-\mathrm{B}(\omega)$, alem disso, suas dimensões físicas, que são $(\mathrm{x} / \omega)$, diferem daquelas dos coeficientes da série de Fourier, que são simplesmente (x). Quanto a sua restrição, uma importante condição é geralmente expressa na forma da inequação (3.30):

$\int_{-\infty}^{+\infty}|x(t)| d t<+\infty$

Significa que pelo menos na teoria clássica, se aplica apenas as funções que decaem para zero quando $|t| \rightarrow \infty$. As equações (3.28) e (3.29), são formas de representar a Transformada inversa de Fourier, segundo Newland (1993), o uso de frequiências negativas é uma forma puramente matemática de simplificar as equações. A Transformada e sua inversa também podem ser feitas na forma complexa, permitindo um modo mais compacto de representação. Para que isto ocorra, é necessário fazer uso das relações:

$\mathrm{e}^{i \omega t}=\cos (\omega \mathrm{t})+i \operatorname{sen}(\omega \mathrm{t})$

$\mathrm{e}^{-i \omega \mathrm{t}}=\cos (\omega \mathrm{t})-i \operatorname{sen}(\omega \mathrm{t})$

E definindo uma relação para os componentes da Transformada de Fourier, tem-se:

$\mathrm{X}(\omega)=\mathrm{A}(\omega)-i \mathrm{~B}(\omega)$

Substituindo as equações (3.26) e (3.27) em (3.33), em seguida usando a relação de (3.32), chega-se:

$X(\omega)=\frac{1}{2 \pi} \int_{-\infty}^{+\infty} x(t) e^{-i \omega t} d t$ 
A equação (3.34) é chamada Transformada de Fourier de x(t). Para a obtenção da forma complexa da Transformada inversa de Fourier, é necessária a integral em um intervalo simétrico do produto entre funções pares e impares, devido a isto se chega as seguintes equações:

$$
\begin{aligned}
& \int_{-\infty}^{+\infty} \mathrm{A}(\omega) \operatorname{sen}(\omega \mathrm{t}) \mathrm{d} \omega=0 \\
& \int_{-\infty}^{+\infty} \mathrm{B}(\omega) \cos (\omega \mathrm{t}) \mathrm{d} \omega=0
\end{aligned}
$$

E para as equações (3.35) e (3.36) define-se a relação:

$$
i \int_{-\infty}^{+\infty} \mathrm{A}(\omega) \operatorname{sen}(\omega \mathrm{t}) \mathrm{d} \omega-i \int_{-\infty}^{+\infty} \mathrm{B}(\omega) \cos (\omega \mathrm{t}) \mathrm{d} \omega=0
$$

Somando (3.37) a equação (3.29), e utilizando a relação de (3.31), chega-se:

$$
\mathrm{x}(\mathrm{t})=\int_{-\infty}^{+\infty} \mathrm{X}(\omega) \mathrm{e}^{i \omega \mathrm{t}} \mathrm{d} \omega
$$

A equação (3.38) é a Transformada inversa de Fourier em sua forma complexa. Como pode ser observado, o sinal em função do tempo fornece a amplitude em função da freqüência e vice-versa. Em momento algum foi possível obter uma relação entre tempo-freqüência, quando o sinal é estacionário esta relação não é importante, pois cada intervalo de tempo possui todas as freqüências do sinal.

No entanto, quando o sinal é não estacionário, pode ocorrer que um ou mais intervalos de tempo não possuam todas as freqüências do sinal, este fato torna a Transformada de Fourier pouco eficiente para este tipo de carregamento. Para minimizar este problema, é utilizada uma técnica de janelamento do sinal no tempo, conhecida como janela tempofreqüência, que significa simplesmente a análise de um intervalo de tempo do sinal, sendo possível dizer quais as freqüências existentes para este intervalo de tempo, ou seja, se estabelece uma relação tempo-freqüência embora que não muito precisa (Figura 3.2(a) e 3.2(b)). 
(a)

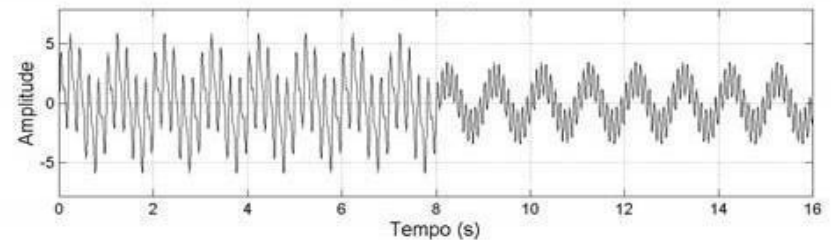

(b)

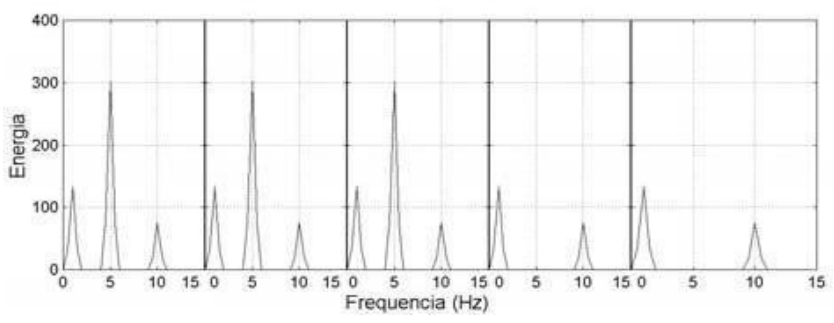

Figura 3.1. Aplicação da transformada janelada: (a) Sinal não estacionário; (b) Espectros de potência do sinal em determinados intervalos de tempo (Imagem modificada a partir de Barbosa; Blitzkow (2008)).

Devido a isto, a partir de (3.34) pode-se realizar a seguinte mudança na transformada de Fourier:

$X G(\omega)=\frac{1}{2 \pi} \int_{-\infty}^{+\infty} x(t) g(t) e^{-i \omega t} d t$

Onde (3.39) é a Transformada janelada de Fourier ou STFT (Short Time Fourier Transform) e $\mathrm{g}(\mathrm{t})$ representa a função da janela, esta pode até ser de forma retangular, no entanto, variações bruscas dos valores da função da janela podem causar o "fenômeno de Gibbs" ou "efeito de borda" que pode ser minimizado com o uso de funções de janelas com decaimento suave. O primeiro pesquisador a utilizar este conceito foi Garbor, o qual definiu para a função da janela:

$\mathrm{g}(\mathrm{t})=\frac{\sqrt{2} \pi}{\alpha} \mathrm{e}^{-\frac{(\mathrm{t}-\beta)^{2}}{4 \alpha}}$

Pode-se notar que (3.40) é proporcional a função distribuição de probabilidade da Gaussiana em (2.3), sendo $\alpha$ referente à largura da janela e $\beta$ o valor médio do intervalo. De acordo com Morettin (1999), em geral, uma transformada janelada de Fourier não conta com a flexibilidade de uma janela tempo-freqüência que aumente os intervalos de tempo para intervalos de baixa freqüência e diminua os intervalos de tempo para intervalos de alta freqüência. Esta flexibilidade será obtida adiante com o uso das wavelets. 


\subsection{ANÁLISE ESPECTRAL}

Para uma representação mais compacta dos coeficientes da série de Fourier, coloca-se os dois tipos de coeficientes em um único gráfico, este gráfico chama-se espectro de amplitude, o espectro de amplitude pode ser representado por freqüências nãonegativas, ou positivas e negativas (Figuras 3.3(a) e 3.3(b)):

a)

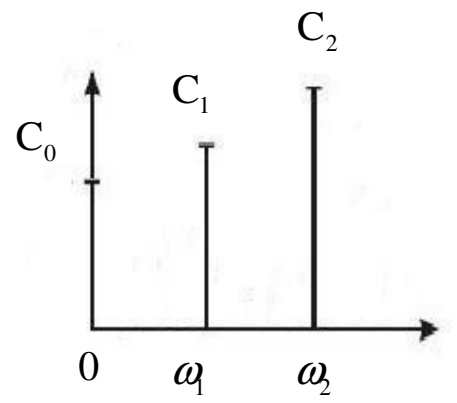

b)

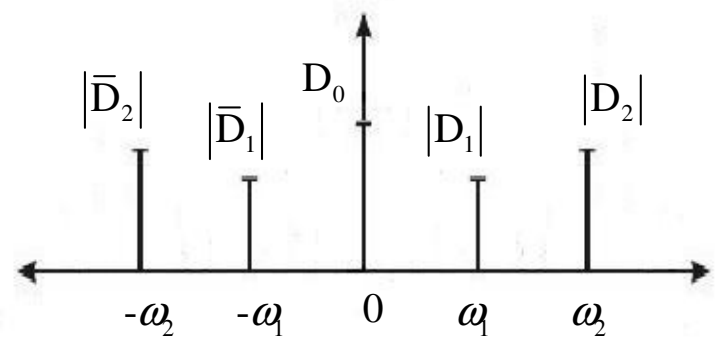

Figura 3.2. Espectro de amplitude: (a) com freqüências não-negativas; (b) com frequiências no domínio dos reais.

É possível obter um espectro que represente a distribuição de energia da série de Fourier em função de sua frequiência, isto é possível devido ao Teorema de Parseval, que usa o princípio da conservação de energia:

$\frac{1}{\mathrm{~T}} \int_{-\mathrm{T} / 2}^{+\mathrm{T} / 2} \mathrm{x}(\mathrm{t})^{2} \mathrm{dt}=\int_{-\infty}^{+\infty}|\mathrm{X}(\omega)|^{2} \mathrm{~d} \omega$

Substituindo (2.7) em (3.41):

$\mathrm{E}\left[\mathrm{x}^{2}\right]=\int_{-\infty}^{+\infty}|\mathrm{X}(\omega)|^{2} \mathrm{~d} \omega$

Segundo Blessmann (1998), a distribuição, nas diversas freqüências, da energia contida em um processo aleatório pode ser representada em um gráfico, chamado de Espectro de potência, este pode ser representado por freqüências não-negativas, ou positivas e negativas (Figura 3.3(a) e 3.3(b)). 
(a)

$$
\mathrm{C}_{2}^{2} / 2
$$

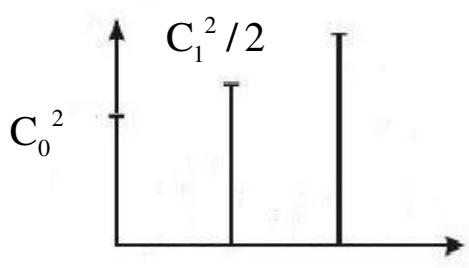

$0 \quad \omega_{1} \quad \omega_{2}$ (b)

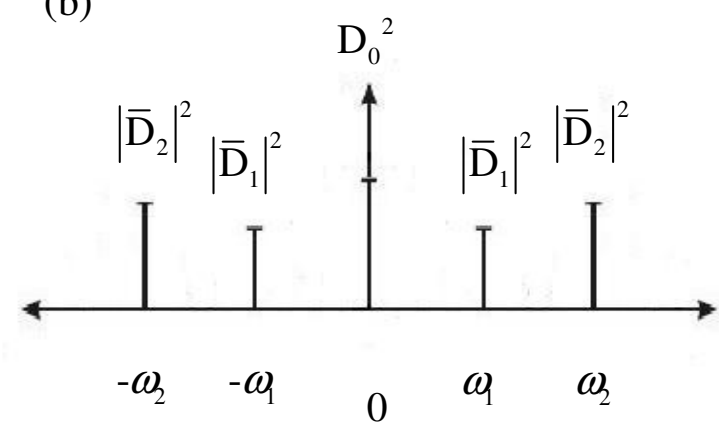

Figura 3.3. Espectros de potência: (a) com freqüências não-negativas; (b) com frequiências positivas e negativas.

Quando as funções são não-periódicas, é equivalente a dizer que a função tem um período T com valor infinito, o que não satisfaz a condição (3.30). Para contornar este problema, utiliza-se a função de autocorrelação, que de acordo com Newland (1993), as freqüências presentes no gráfico de $\mathrm{R}_{\mathrm{x}}(\tau)$ contra $\tau$ reflete as freqüências contidas na função $\mathrm{x}(\mathrm{t})$, com a propriedade de quando $\tau \rightarrow \infty, \mathrm{R}_{\mathrm{x}}(\tau) \rightarrow 0$, resultando em:

$\int_{-\infty}^{+\infty}\left|\mathrm{R}_{\mathrm{x}}(\tau)\right| \mathrm{dt}<+\infty$

Satisfazendo a inequação (3.30). Então, pode-se aplicar a transformada de Fourier na função de autocorrelação, obtendo-se:

$\mathrm{S}_{\mathrm{x}}(\omega)=\frac{1}{2 \pi} \int_{-\infty}^{+\infty} \mathrm{R}_{\mathrm{x}}(\tau) \mathrm{e}^{-i \omega \tau} \mathrm{d} \tau$

Sendo sua transformada inversa de Fourier apresentada como:

$$
\mathrm{R}_{\mathrm{x}}(\tau)=\int_{-\infty}^{+\infty} \mathrm{S}_{\mathrm{x}}(\omega) \mathrm{e}^{i \omega \tau} \mathrm{d} \omega
$$

Onde (3.44) é chamada de Densidade espectral de x(t) e de acordo com Newland (1993), sua denominação mais completa é Densidade espectral do quadrado médio (mean square spectral density), mais conhecida como Função de densidade espectral de Potência ou PSDF (Power Spectral Density Function). Quanto a (3.45), segundo Newland (1993), a propriedade mais importante se torna aparente quando $\tau=0$ :

$$
\mathrm{R}_{\mathrm{x}}(0)=\int_{-\infty}^{+\infty} \mathrm{S}_{\mathrm{x}}(\omega) \mathrm{d} \omega
$$


Aonde, de acordo com (2.29), tem-se:

$$
\mathrm{E}\left[\mathrm{x}^{2}\right]=\int_{-\infty}^{+\infty} \mathrm{S}_{\mathrm{x}}(\omega) \mathrm{d} \omega
$$

No processo estacionário, o valor quadrado médio coincide com a área sob a função de Densidade Espectral, então, a dimensão física de (3.44) é $\left(\mathrm{x}^{2} / \omega\right)$. Utilizando a mesma relação que (3.33) para a densidade espectral, tem-se:

$\mathrm{S}_{\mathrm{x}}(\omega)=\mathrm{A}(\omega)-i \mathrm{~B}(\omega)$

Sendo os coeficientes da Densidade Espectral gerados a partir de (3.26) e (3.27), apenas substituindo a função $\mathrm{x}(\mathrm{t})$ por $\mathrm{R}_{\mathrm{x}}(\tau)$ :

$$
\begin{aligned}
& \mathrm{A}(\omega)=\frac{1}{2 \pi} \int_{-\infty}^{+\infty} \mathrm{R}_{\mathrm{x}}(\tau) \cos (\omega \mathrm{t}) \mathrm{dt} \\
& \mathrm{B}(\omega)=\frac{1}{2 \pi} \int_{-\infty}^{+\infty} \mathrm{R}_{\mathrm{x}}(\tau) \operatorname{sen}(\omega \mathrm{t}) \mathrm{dt}
\end{aligned}
$$

Percebe-se em (3.50) que a função de autocorrelação, uma função par, está sendo multiplicada pelo seno, que é uma função impar em uma integral com um intervalo simétrico, o que ocasiona um valor nulo para (3.50), assim sendo, a relação (3.48), torna-se:

$\mathrm{S}_{\mathrm{x}}(\omega)=\mathrm{A}(\omega)$

Além de (3.51) mostrar que a densidade espectral para um processo estacionário é uma função par, real e não-negativa, esta afirmação pode ser reforçada pela equação (3.47), onde a área sob a curva de Densidade Espectral é equivalente ao seu valor quadrado médio. É comum a utilização de formas alternativas para a equação (3.47), uma forma bastante utilizada é o uso de freqüências lineares não-negativas. Para encontrar uma relação entre estas duas representações da Densidade Espectral, usa-se o princípio de cálculo da área:

$\mathrm{E}\left[\mathrm{x}^{2}\right]=\int_{0}^{+\infty} \mathrm{W}_{\mathrm{x}}(f) \mathrm{d} f$ 
Onde $\mathrm{W}_{\mathrm{x}}(f)$ representa a Densidade Espectral, igualando a equação (3.47) com (3.52) e utilizando a relação:

$f=\frac{\omega}{2 \pi}$

Fazendo um elemento diferencial de (3.53) e igualando os domínios das integrais, temse:

$2 \int_{0}^{+\infty} \mathrm{S}_{\mathrm{x}}(\omega) \mathrm{d} \omega=\int_{0}^{+\infty} \mathrm{W}_{\mathrm{x}}\left(\frac{\omega}{2 \pi}\right) \frac{\mathrm{d} \omega}{2 \pi}$

O que é equivalente a:

$\mathrm{W}_{\mathrm{x}}(f)=4 \pi \mathrm{S}_{\mathrm{x}}(\omega)$

Uma relação que terá importância neste trabalho é como obter um valor de amplitude em um intervalo $\Delta \omega$ através da função de Densidade Espectral, isto pode ser feito igualando (3.42) a (3.47), e utilizando um intervalo $\Delta \omega$ para a integração:

$\int_{\omega}^{\omega+\Delta \omega}|\mathrm{X}(\omega)|^{2} \mathrm{~d} \omega=\int_{\omega}^{\omega+\Delta \omega} \mathrm{S}_{\mathrm{x}}(\omega) \mathrm{d} \omega$

Utilizando (3.12) para (3.26) e (3.27), tem-se :

$\mathrm{C}^{2}=\int_{\omega}^{\omega+\Delta \omega}|\mathrm{X}(\omega)|^{2} \mathrm{~d} \omega=\int_{\omega}^{\omega+\Delta \omega} \mathrm{A}(\omega)^{2}+\mathrm{B}(\omega)^{2} \mathrm{~d} \omega$

Igualando a (3.56) e novamente com alguma manipulação algébrica:

$\mathrm{C}_{\Delta \omega}=\sqrt{\int_{\omega}^{\omega+\Delta \omega} \mathrm{S}_{\mathrm{x}}(\omega) \mathrm{d} \omega}$

Para funções de densidade espectrais com freqüências não-negativas, como $\mathrm{W}_{\mathrm{x}}(f)$, a amplitude obtida tem o dobro do valor, como a relação é quadrática, o valor se torna quatro vezes maior, o que resulta em:

$\mathrm{C}_{\Delta f}=\sqrt{2 \int_{f}^{f+\Delta f} \mathrm{~W}_{\mathrm{x}}(f) \mathrm{d} f}$

A equação (3.59) é bastante utilizada para processos estocásticos de geração de carregamento. 


\subsection{ANÁLISE NUMÉRICA DE FOURIER}

Para uma análise computacional é necessária uma partição do intervalo e a limitação do tempo de ocorrência do sinal. Em teoria, para sinais não-periódicos, a geração da transformada necessita da consideração de um período $|\mathrm{T}| \rightarrow+\infty$ e um intervalo de freqüência angular $\Delta \omega \rightarrow \mathrm{d} \omega$.

No entanto, Newland (1993) diz que a palavra "série temporal" é também usada para se referir a seqüência de números discretos $\mathrm{x}_{\mathrm{r}}$ ordenados no tempo, ou a uma amostra temporal $\mathrm{x}(\mathrm{t})$, originalmente contínua, a qual uma série discreta pode ser obtida (Figura $3.4)$.

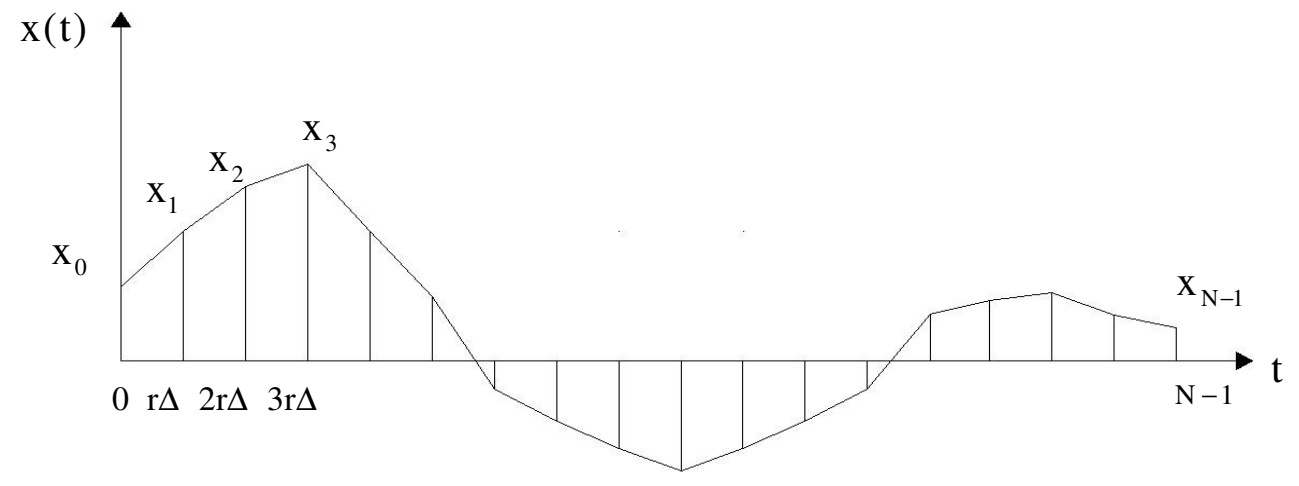

Figura 3.4. Série discreta obtida através de uma série temporal contínua.

Uma boa definição é feita por Chierice (2007), onde explica que uma série temporal não corresponde a uma função contínua e infinita, e sim a um conjunto discreto de valores que muitas vezes se obtêm por meio da simulação computacional de um modelo matemático. A partição pode ser obtida a partir da relação (3.33), pois ao invés do uso das componentes da Transformada de Fourier, são usados os coeficientes da série de Fourier devido ao fato destes serem elementos discretos.

$\mathrm{X}_{\mathrm{k}}=a_{\mathrm{k}}-i b_{\mathrm{k}}$

Substituindo as equações (3.3) e (3.4) em (3.60), em seguida usando a relação (3.32) de forma discreta em relação a frequiência angular, e deslocando o domínio da integral em $+\mathrm{T} / 2$, chega-se:

$$
\mathrm{X}_{\mathrm{k}}=\frac{1}{\mathrm{~T}} \int_{0}^{\mathrm{T}} \mathrm{x}(\mathrm{t}) \mathrm{e}^{-i \omega_{k} \mathrm{t}} \mathrm{dt} \quad \text { para } \quad \mathrm{k} \geq 0
$$


O domínio é deslocado por questão de simplificação de cálculo, o deslocamento em T/2 mantendo o mesmo resultado é possível devido ao fato da função ser periódica. Agora considerando que a série temporal $\mathrm{x}(\mathrm{t})$, sendo contínua não é conhecida, apenas pontos espaçados igualmente, estes pontos representam uma série discreta $\mathrm{x}_{\mathrm{r}}$, onde $\mathrm{r}$ pode variar de 0 a $(\mathrm{N}-1)$, considerando $\Delta$ o espaçamento no tempo dos pontos e $\mathrm{N}$ o número de pontos, tem-se:

$$
\begin{array}{ll}
\mathrm{t}=\mathrm{r} \Delta & \text { para } \quad \mathrm{r}=0,1, \ldots,(\mathrm{N}-1) \\
\mathrm{T}=\mathrm{N} \Delta &
\end{array}
$$

Alterando a integral pelo somatório, $\mathrm{x}(\mathrm{t})$ por $\mathrm{x}_{\mathrm{r}}$, e substituindo (3.8), (3.62) e (3.63) em (3.61), tem-se:

$$
\mathrm{X}_{\mathrm{k}}=\frac{1}{\mathrm{~N}} \sum_{\mathrm{r}=0}^{\mathrm{N}-1} \mathrm{x}_{\mathrm{r}} \mathrm{e}^{-i(2 \pi \mathrm{kr} / \mathrm{N})} \quad \text { para } \quad \mathrm{k}=0,1, \ldots,(\mathrm{N}-1)
$$

Onde (3.64) é chamada de Transformada Discreta de Fourier ou DFT (Discrete Fourier Transform), Para a Transformada Discreta inversa de Fourier, o processo é similar a obtenção da Transformada inversa de Fourier, pois ao invés do uso das componentes da Transformada de Fourier e integrais, são usados os coeficientes da série de Fourier e somatórios. Devido a isto, é definida a relação:

$$
i \sum_{\mathrm{k}=0}^{\mathrm{N}-1}\left[a_{\mathrm{k}} \operatorname{sen}\left(\omega_{\mathrm{k}} \mathrm{t}\right)-b_{\mathrm{k}} \cos \left(\omega_{\mathrm{k}} \mathrm{t}\right)\right]=0
$$

E a série de Fourier pode ser escrita na forma:

$$
\mathrm{x}_{\mathrm{r}}=\sum_{\mathrm{k}=0}^{\mathrm{N}-1}\left[a_{\mathrm{k}} \cos \left(\omega_{\mathrm{k}} \mathrm{t}\right)+b_{\mathrm{k}} \operatorname{sen}\left(\omega_{\mathrm{k}} \mathrm{t}\right)\right] \quad \text { para } \quad \mathrm{r}=0,1, \ldots,(\mathrm{N}-1)
$$

Sendo a função do somatório substituir uma integral com domínio entre 0 e $\omega=2 \pi / \mathrm{T}$ com isto se pode notar que o domínio da transformada inversa depende do domínio da transformada, permitindo a mesma quantidades de pontos analisados. Somando (3.65) a (3.66) e utilizando (3.31) com a freqüência em valores discretos, juntamente com (3.8), (3.62) e (3.63), chega-se: 
$\mathrm{X}_{\mathrm{r}}=\sum_{\mathrm{k}=0}^{\mathrm{N}-1} \mathrm{X}_{\mathrm{k}} \mathrm{e}^{i(2 \pi \mathrm{kr} / \mathrm{N})} \quad \mathrm{para} \quad \mathrm{r}=0,1, \ldots,(\mathrm{N}-1)$

Onde (3.67) é chamada de Transformada Discreta inversa de Fourier ou IDFT (Inverse Discrete Fourier Transform). Quando o sinal tem comportamento não estacionário, pode ser utilizada uma técnica de janelamento do sinal no tempo de uma forma particionada. Então, a partir de (3.39) chega-se:

$\mathrm{XG}_{\mathrm{k}}=\frac{1}{\mathrm{~N}} \sum_{\mathrm{r}=0}^{\mathrm{N}-1} \mathrm{x}_{\mathrm{r}} \mathrm{g}_{\mathrm{r}} \mathrm{e}^{-i(2 \pi \mathrm{kr} / \mathrm{N})} \quad$ para $\quad \mathrm{k}=0,1, \ldots,(\mathrm{N}-1)$

Onde (3.68) é a Transformada Discreta Janelada de Fourier ou DSTFT (Discrete Short Time Fourier Transform) e $\mathrm{g}_{\mathrm{r}}$ representa a função da janela em sua forma discreta. Agora, considerando a partição da autocorrelação, a defasagem de tempo $\tau$ é dada por: $\tau=\mathrm{r} \Delta \quad \mathrm{para} \quad \mathrm{r}=0,1, \ldots,(\mathrm{N}-1)$

Como pode ser observado, para a obtenção de cada coeficiente da DFT, são necessários o somatório de $\mathrm{N}$ produtos e para a obtenção da DFT são necessário $\mathrm{N}$ coeficientes, no total, ocorrem $\mathrm{N}^{2}$ operações, resultando em varias truncagems de produtos devido ao numero limitado de casas decimais do computador, além de uma demanda de tempo considerável para séries temporais com muitos pontos, mesmo com a capacidade de processamento atual.

Com a intenção de minimização destes problemas, foi criado um algoritmo, chamado de Fast Fourier Transform ou FFT (Transformada rápida de Fourier), esta trabalha com a partição de uma série inteira em um número de séries cada vez menores, ocasionando o cálculo da DFT em paralelo destas séries particionadas, resultando em uma redução do tempo e um aumento de precisão.

Agora, retornando a DFT, segundo a dedução apresentada por Newland (1993), a primeira etapa é a separação em duas partes, uma onde os índices de $\mathrm{x}_{\mathrm{r}}$ são pares e outra onde são impares:

$\mathrm{X}_{\mathrm{k}}=\frac{1}{\mathrm{~N}}\left[\sum_{\mathrm{r}=0}^{\mathrm{N} / 2-1} \mathrm{x}_{2 \mathrm{r}} \mathrm{e}^{-i(2 \pi \mathrm{k}(2 \mathrm{r}) / \mathrm{N})}+\sum_{\mathrm{r}=0}^{\mathrm{N} / 2-1} \mathrm{x}_{2 \mathrm{r}+1} \mathrm{e}^{-i(2 \pi \mathrm{k}(2 \mathrm{r}+1) / \mathrm{N})}\right]$ para $\mathrm{k}=0,1, \ldots,(\mathrm{N}-1)$ 
Onde, por definição:

$$
\begin{array}{lll}
\mathrm{x}_{\mathrm{r}}^{\mathrm{e}}=\mathrm{X}_{2 \mathrm{r}} & \text { para } & \mathrm{r}=0,1, \ldots,(\mathrm{N} / 2-1) \\
\mathrm{X}_{\mathrm{r}}^{\mathrm{o}}=\mathrm{x}_{2 \mathrm{r}+1} & \text { para } & \mathrm{r}=0,1, \ldots,(\mathrm{N} / 2-1) \\
\mathrm{X}_{\mathrm{k}}^{\mathrm{e}}=\frac{1}{(\mathrm{~N} / 2)} \sum_{\mathrm{r}=0}^{\mathrm{N} / 2-1} \mathrm{x}^{\mathrm{e}}{ }_{\mathrm{r}} \mathrm{e}^{-i(2 \pi \mathrm{kr} /(\mathrm{N} / 2))} & \text { para } & \mathrm{k}=0,1, \ldots,(\mathrm{N} / 2-1) \\
\mathrm{X}_{\mathrm{k}}^{\mathrm{o}}=\frac{1}{(\mathrm{~N} / 2)} \sum_{\mathrm{r}=0}^{\mathrm{N} / 2-1} \mathrm{x}^{\mathrm{o}}{ }_{\mathrm{r}} \mathrm{e}^{-i(2 \pi \mathrm{kr} /(\mathrm{N} / 2))} & \text { para } & \mathrm{k}=0,1, \ldots,(\mathrm{N} / 2-1)
\end{array}
$$

Como $\mathrm{X}_{\mathrm{k}}^{\mathrm{e}}$ e $\mathrm{X}_{\mathrm{k}}^{\mathrm{o}}$ tem o período de $\mathrm{N} / 2$, os valores se repetem a cada $\mathrm{N} / 2$ vezes, resultando em:

$$
\begin{array}{lll}
\mathrm{X}_{\mathrm{k}+\mathrm{N} / 2}^{\mathrm{e}}=\mathrm{X}_{\mathrm{k}}^{\mathrm{e}} & \text { para } & \mathrm{k}=0,1, \ldots,(\mathrm{N} / 2-1) \\
\mathrm{X}^{\mathrm{o}}{ }_{\mathrm{k}+\mathrm{N} / 2}=\mathrm{X}_{\mathrm{k}}^{\mathrm{o}} & \text { para } & \mathrm{k}=0,1, \ldots,(\mathrm{N} / 2-1)
\end{array}
$$

Substituindo (3.73) e (3.74) em (3.70), chega-se:

$$
\begin{array}{lll}
\mathrm{X}_{\mathrm{k}}=\frac{1}{2}\left[\mathrm{X}_{\mathrm{k}}^{\mathrm{e}}+\mathrm{W}_{\mathrm{k}} \mathrm{X}_{\mathrm{k}}^{\mathrm{o}}\right] & \text { para } & \mathrm{k}=0,1, \ldots,(\mathrm{N} / 2-1) \\
\mathrm{X}_{\mathrm{k}+\mathrm{N} / 2}=\frac{1}{2}\left[\mathrm{X}_{\mathrm{k}}^{\mathrm{e}}-\mathrm{W}_{\mathrm{k}} \mathrm{X}_{\mathrm{k}}^{\mathrm{o}}\right] & \text { para } & \mathrm{k}=0,1, \ldots,(\mathrm{N} / 2-1)
\end{array}
$$

Onde:

$$
\mathrm{W}_{\mathrm{k}}=\mathrm{e}^{-\mathrm{i}(2 \pi \mathrm{k} / \mathrm{N})}
$$

Para (3.77) e (3.78) o processo se repete, separam-se os índices pares e impares de $\mathrm{X}_{\mathrm{k}}^{\mathrm{e}}$ e $\mathrm{X}_{\mathrm{k}}^{\mathrm{o}}$, e cada uma destes resultados são divididos na mesma forma, até chegar ao ponto onde cada $\mathrm{X}_{\mathrm{k}}^{\mathrm{e}}$ e $\mathrm{X}_{\mathrm{k}}^{\mathrm{o}}$ seja apenas gerado por um ponto do sinal, a partir disto começa a aplicação da FFT, que é justamente o processo inverso da sua dedução. 
A FFT reduz o número de produtos para $\mathrm{N} \log _{2} \mathrm{~N}$, aumentando a precisão dos coeficientes da DFT e reduzindo o tempo de processamento. Uma desvantagem do método é que a série apenas pode utilizar uma quantidade de pontos iguais a uma potência de 2 , pois a série é dividida pela metade, depois em um quarto e assim por diante, até chegar em um único número. No entanto, esta imposição de quantidade de valores pode ser contornada com o uso de truncamento dos pontos ou inclusão de pontos de valor igual à zero no sinal analisado. 


\section{ANÁLISE DE WAVELET}

\subsection{INTRODUÇÃO}

A wavelet ou ondaleta, como sugerida por Morettin (1999), é uma função capaz de descrever e decompor funções no domínio da freqüência de forma a ser possível a análise em diferentes escalas de frequiência e de tempo. Uma das grandes diferenças da análise de wavelet em relação a análise de Fourier é que na primeira há uma concentração de energia em uma região finita, já que a ultima usa as funções de seno e cosseno que são periódicas.

Outra diferença é que na análise de Fourier pode-se extrair com bastante precisão informações sobre o domínio da freqüência e razoável precisão no tempo com o uso do conceito de janela espectral, este de acordo com Morettin (1999), trabalha com uma janela fixa no domínio do tempo e da frequiência, o que torna difícil a captura de altas e baixas frequencia de um sinal simultaneamente, sendo este problema resolvido na análise com wavelets.

Entretanto a maioria das wavelets não são tão bem localizadas no domínio da freqüência como as funções trigonométricas que formam a base da análise de Fourier. Em uma comparação ao princípio da incerteza de Heisenberg, chama-se a relação entre os domínios da freqüência e do tempo de relação de incerteza ou simplesmente de princípio da incerteza.

No geral, pode-se dizer que as wavelets fornecem uma forma de balancear a incerteza entre o domínio do tempo e o domínio da freqüência. Neste trabalho as wavelets constituem um importante método, pois são funções de comportamento oscilatório, média nula e energia finita, sendo bastante útil na representação de processos estocásticos com comportamento não-estacionário. 


\subsection{DEFINIÇÃO DE WAVELET}

Segundo Campanha (2004), uma wavelet ou ondaleta nada mais é do que uma pequena onda, enquanto a análise de Fourier consiste em decompor um sinal em senos e cossenos de várias freqüências, a análise de wavelet é a decomposição de um sinal por mudanças de escalas (dilatações e compressões) e translações de uma wavelet original, sendo a wavelet modificada definida como wavelet-filha enquanto a wavelet original definida como wavelet mãe.

(a)

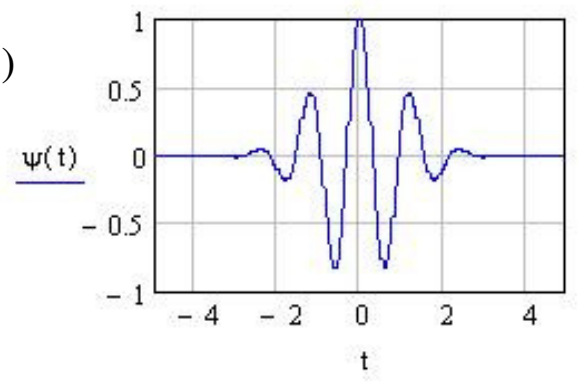

(b)

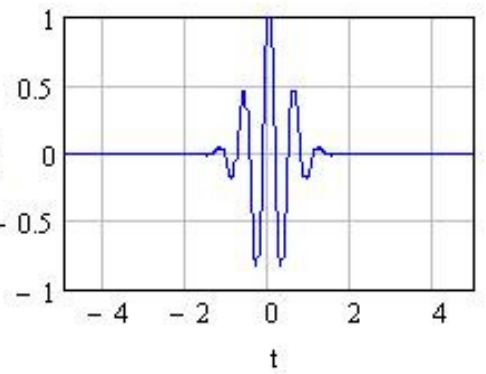

Figura 4.1. Wavelet: (a) wavelet mãe; (b) wavelet filha com mudança de escala.

(a)

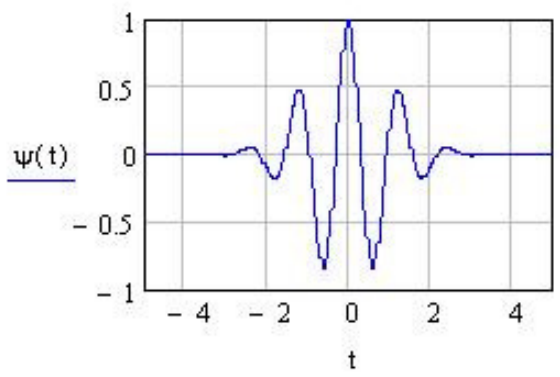

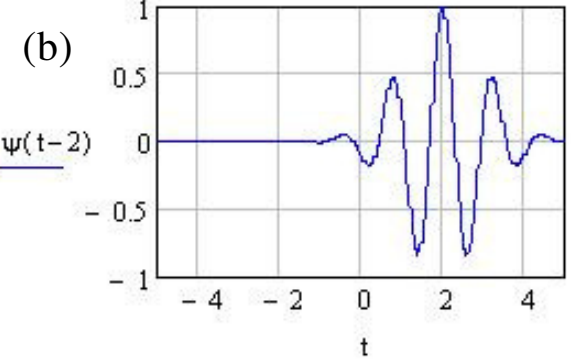

Figura 4.2. Wavelet: (a) wavelet mãe; (b) wavelet filha com mudança de translação.

Geralmente uma wavelet é expressa por uma função $\psi(\mathrm{t})$, esta não precisa ser suave nem simétrica, mas é necessário que esta função pertença ao espaço funcional das funções de quadrado integrável, cobrindo o domínio dos reais $L^{2}(R)$ :

$\int_{-\infty}^{+\infty}|\mathrm{x}(\mathrm{t})|^{2} \mathrm{dt}<+\infty$

O que significa que os valores das funções devem decair para zero quando $|t| \rightarrow+\infty$, fazendo com que a wavelet escolhida decaia rapidamente, pois quanto mais compactada for esta, menor será a influência de uma wavelet filha em sua adjacente para a reconstrução do sinal. A relação (4.1) leva a uma das características mais importantes da análise de wavelet que é sua limitação energética, o que induz a: 
$\int_{-\infty}^{+\infty}|\psi(\mathrm{t})| \mathrm{dt}<+\infty$

Por definição, para a função ser considerada uma onda é necessário que sua média seja igual à zero:

$\int_{-\infty}^{+\infty} \psi(\mathrm{t}) \mathrm{dt}=0$

As condições (4.2) e (4.3) podem também ser satisfeitas através da condição de admissibilidade, para isto é necessário:

$\Psi(\omega)=\int_{-\infty}^{+\infty} \psi(\mathrm{t}) \mathrm{e}^{-i \omega t} \mathrm{dt}$

Como (4.4) é a transformada de Fourier da wavelet, sendo esta válida devido a relação (4.2), chega-se:

$\mathrm{C}_{\psi}=\int_{-\infty}^{+\infty} \frac{|\Psi(\omega)|^{2}}{|\omega|} \mathrm{d} \omega<+\infty$

Sendo (4.5) o coeficiente de admissibilidade, o qual garante a existência da transformada de wavelet inversa, este mostra que a wavelet necessita ter energia finita, pois a transformada tende a zero quando $|\omega| \rightarrow+\infty$, e que a transformada em $\omega=0$ necessita ser zero para que (4.5) tenha um valor finito, como o valor da transformada de Fourier em $\omega=0$ representa o valor médio da função, a condição (4.3) é satisfeita.

$\mathrm{Na}$ análise de Fourier, a geração do sinal é feita através das dilatações de funções senos e cosenos ocasionadas pelas variações das frequiências, no entanto, a análise de Fourier é recomendada para séries temporais com comportamento estacionário. Para comportamentos não-estacionários, uma melhor representação é feita pelas wavelets por meio de mudanças de escala e translações, então a wavelet filha fica em relação à wavelet mãe na forma:

$\psi_{\alpha, \beta}(\mathrm{t})=|\alpha|^{-1 / 2} \psi\left(\frac{\mathrm{x}-\beta}{\alpha}\right) \quad$ para $\alpha, \beta \in \mathrm{R}, \alpha \neq 0$ 
Equivalente a Transformada janelada de Fourier, $\alpha$ é o coeficiente da mudança na escala da função e $\beta$ o coeficiente de sua translação, o inverso da raiz quadrada de $\alpha$ é aplicado nas wavelets filhas para que todas tenham a mesma energia. Para a obtenção da transformada wavelet é necessário que ela possua uma janela no tempo flexível, diferentemente da transformada janelada de Fourier. Então através da correlação entre (4.6) e um sinal $\mathrm{x}(\mathrm{t})$ contínuo que pertence a $L^{2}(R)$, chega-se:

$$
\mathrm{w}(\alpha, \beta)=\int_{-\infty}^{+\infty} \mathrm{x}(\mathrm{t}) \psi_{\alpha, \beta}(\mathrm{t}) \mathrm{dt} \quad \text { para } \alpha, \beta \in \mathrm{R}, \alpha \neq 0
$$

Sendo (4.7) a transformada de wavelet. De acordo com Chiann (1997) a transformada de wavelet fornece uma janela tempo-frequiência flexível a qual automaticamente se estreita quando observa-se o comportamento do sinal em alta freqüência (pequeno valor de $\alpha$ ) e se amplia quando observa-se o comportamento de baixa freqüência (grande valor de $\alpha$ ).

Para a reconstrução do sinal tem-se a inversa da transformada de wavelet, esta pode ser obtida a partir do teorema de Parseval, onde de acordo com Chiann (1997), o teorema também é válido para wavelets, portanto, a energia também se conserva na transformada de wavelet:

$$
\int_{-\infty}^{+\infty} \mathrm{x}(\mathrm{t})^{2} \mathrm{dt}=\frac{1}{\mathrm{C}_{\psi}} \int_{-\infty}^{+\infty} \int_{-\infty}^{+\infty}|\mathrm{w}(\alpha, \beta)|^{2} \mathrm{~d} \alpha \mathrm{d} \beta \quad \text { para } \alpha, \beta \in \mathrm{R}, \alpha \neq 0
$$

Desde que obedeça a condição (4.5), a transformada inversa de wavelet pode ser obtida com a equação que representa o sinal $\mathrm{x}(\mathrm{t})$ pertencente a $L^{2}(R)$, de forma que a aplicação do lado esquerdo da equação (4.8) resulte no lado direito da mesma, devido a isto, chega-se:

$$
\mathrm{x}(\mathrm{t})=\frac{1}{\mathrm{C}_{\psi}} \int_{-\infty}^{+\infty} \int_{-\infty}^{+\infty} \mathrm{w}(\alpha, \beta) \psi_{\alpha, \beta}(\mathrm{t}) \frac{\mathrm{d} \alpha \mathrm{d} \beta}{\alpha^{2}} \quad \text { para } \alpha, \beta \in \mathrm{R}, \alpha \neq 0
$$

Usualmente os valores dos coeficientes $\alpha$ e $\beta$ são dados por valores racionais, sendo seus valores mais comuns expressos por:

$$
\begin{array}{lll}
\alpha=2^{-\mathrm{j}} & \text { para } & \mathrm{j} \in \mathrm{Z} \\
\beta=\mathrm{k} 2^{-\mathrm{j}} & \text { para } & \mathrm{j}, \mathrm{k} \in \mathrm{Z}
\end{array}
$$


Então as wavelets filhas podem ser obtidas através de escalas binárias em (4.10) e translações diádicas em (4.11) como definidas por Morettin (1999). Substituindo estas duas ultimas relações em (4.6), tem-se:

$\psi_{\mathrm{j}, \mathrm{k}}(\mathrm{t})=2^{\mathrm{j} / 2} \psi\left(2^{\mathrm{j}} \mathrm{x}-\mathrm{k}\right)$

As wavelets filhas formam uma base, que segundo Morettin (1999), não precisa ser ortogonal, porem, a vantagem de se trabalhar com bases ortogonais é que elas permitem a geração perfeita do sinal a partir dos coeficientes da transformada, uma transformada ortogonal, como aquela usada por senos e cossenos, é compacta, sendo cada coeficiente calculado como produto interno do sinal com a função da base $\psi(\mathrm{t})$, como indicado nas equações (4.13) e (4.14), para uma facilitação do cálculo foi introduzida a normalidade na função base, gerando assim, uma base ortonormal:

$$
\begin{aligned}
& \left\langle\psi_{\mathrm{j}_{1}, \mathrm{k}_{1}}, \psi_{\mathrm{j}_{2}, \mathrm{k}_{2}}\right\rangle=\int_{-\infty}^{+\infty} \psi_{\mathrm{j}_{1}, \mathrm{k}_{1}}(\mathrm{t}) \psi_{\mathrm{j}_{2}, \mathrm{k}_{2}}(\mathrm{t}) \mathrm{dt}=0 \quad \text { para } \mathrm{j}_{1} \neq \mathrm{j}_{2} \text { ou } \mathrm{k}_{1} \neq \mathrm{k}_{2} \\
& \left\langle\psi_{\mathrm{j}, \mathrm{k}}, \psi_{\mathrm{j}, \mathrm{k}}\right\rangle=\int_{-\infty}^{+\infty} \psi_{\mathrm{j}, \mathrm{k}}(\mathrm{t}) \psi_{\mathrm{j}, \mathrm{k}}(\mathrm{t}) \mathrm{dt}=\int_{-\infty}^{+\infty} \psi_{\mathrm{j}, \mathrm{k}}(\mathrm{t})^{2} \mathrm{dt}=1
\end{aligned}
$$

A partir das relações (4.10) e (4.11) e do uso de wavelets ortogonais a correlação do sinal com a wavelet pode ser modificada para seu caso discreto, então, a partir de (4.7) chega-se:

$$
w(j, k)=\int_{-\infty}^{+\infty} x(t) \psi_{j, k}(t) d t=\left\langle x, \psi_{j, k}\right\rangle=w_{j, k}
$$

Com isto, o sinal $\mathrm{x}(\mathrm{t})$ pode ser reconstruído por:

$$
\mathrm{x}(\mathrm{t})=\sum_{\mathrm{j}=-\infty}^{+\infty} \sum_{\mathrm{k}=-\infty}^{+\infty}\left\langle\mathrm{x}, \psi_{\mathrm{j}, \mathrm{k}}\right\rangle \psi_{\mathrm{j}, \mathrm{k}}(\mathrm{x})=\sum_{\mathrm{j}=-\infty}^{+\infty} \sum_{\mathrm{k}=-\infty}^{+\infty} \mathrm{w}_{\mathrm{j}, \mathrm{k}} \psi_{\mathrm{j}, \mathrm{k}}(\mathrm{x})
$$

Onde (4.16) é a transformada inversa de wavelets ortogonais ou ortonormais. Pode-se observar pela condição de admissibilidade em (4.5) que todas as wavelets tem média nula, não sendo possível a geração de sinais de valores médios diferentes de zero, para corrigir este problema é aplicada uma função que supra esta necessidade, chamada de função de escala ou wavelet pai, embora não seja uma wavelet, pois a condição (4.3) não é satisfeita, esta condição é substituída por: 
$\int_{-\infty}^{+\infty} \phi(\mathrm{t}) \mathrm{dt}=1$

Sendo a função de escala geralmente expressa por $\phi(t)$, com sua função na forma:

$\phi_{\gamma}(\mathrm{t})=\phi(\mathrm{t}-\gamma) \quad$ para $\quad \gamma \in \mathrm{R}$

Com $\gamma$ sendo o coeficiente de sua translação. Não há uma definição geral quanto a utilização de uma mudança de escala neste tipo de função, o consenso que existe é que as wavelets representam melhor as partes de mudanças bruscas e de alta freqüência de um sinal, e as funções de escala representam melhor partes suaves e de baixa freqüência em um sinal, devido a isto possui uma janela tempo-frequência ampliada, sendo muitas vezes representadas por coeficientes de escala de valores negativos, Newland (1993) representa as funções de escala ortogonais com coeficiente de dilatação do valor de -1 por razão representativa.

Então as funções de escala filhas podem ser obtidas através de translações diádicas sem mudança de escala, devido a isto, impondo valores inteiros para $\gamma$ :

$\gamma=\mathrm{k}$

E substituindo esta ultima relação em (4.18), tem-se:

$\phi_{\mathrm{k}}(\mathrm{t})=\phi(\mathrm{t}-\mathrm{k})$

Considerando que estas funções formam uma base ortonormal, chega-se:

$\left\langle\phi_{\mathrm{k}_{1}}, \phi_{\mathrm{k}_{2}}\right\rangle=\int_{-\infty}^{+\infty} \phi_{\mathrm{k}_{1}}(\mathrm{t}) \phi_{\mathrm{k}_{2}}(\mathrm{t}) \mathrm{dt}=0 \quad$ para $\quad \mathrm{k}_{1} \neq \mathrm{k}_{2}$

$\left\langle\phi_{\mathrm{k}}, \phi_{\mathrm{k}}\right\rangle=\int_{-\infty}^{+\infty} \phi_{\mathrm{k}}(\mathrm{t}) \phi_{\mathrm{k}}(\mathrm{t}) \mathrm{dt}=\int_{-\infty}^{+\infty} \phi_{\mathrm{k}}(\mathrm{t})^{2} \mathrm{dt}=1$

A partir da relação (4.20) e do uso de funções de escala ortogonais, a correlação do sinal com a função de escala pode ser modificada para seu caso discreto, então, a partir de (4.7) chega-se:

$\mathrm{u}(\mathrm{k})=\int_{-\infty}^{+\infty} \mathrm{x}(\mathrm{t}) \phi_{\mathrm{k}}(\mathrm{t}) \mathrm{dt}=\left\langle\mathrm{x}, \phi_{\mathrm{k}}\right\rangle=\mathrm{u}_{\mathrm{k}}$ 
Combinando as wavelets com as funções de escala, o sinal $\mathrm{x}(\mathrm{t})$ pode ser reconstruído mesmo se este tiver um valor médio diferente de zero. Adicionando o termo referente à função de escala em (4.16), tem-se:

$\mathrm{x}(\mathrm{t})=\sum_{\mathrm{k}=-\infty}^{+\infty} \mathrm{u}_{\mathrm{k}} \phi_{\mathrm{k}}(\mathrm{t})+\sum_{\mathrm{j}=-\infty}^{+\infty} \sum_{\mathrm{k}=-\infty}^{+\infty} \mathrm{w}_{\mathrm{j}, \mathrm{k}} \psi_{\mathrm{j}, \mathrm{k}}(\mathrm{x})$

Onde (4.24) representa a forma discreta completa da transformada inversa de wavelet. 


\subsection{TIPOS DE WAVELETS}

Diferentemente da análise de Fourier, que trabalha apenas com funções trigonométricas, na análise de wavelets é possível utilizar vários tipos de funções desde que estas respeitem as definições apresentadas anteriormente.

A escolha da wavelet dependerá da necessidade do pesquisador, como por exemplo, se a forma da função refletir as características da série temporal, sintetizar dados e fazer compressões ou obter informações quantitativas, para estes, é interessante o uso de wavelets ortogonais; para redundância de informações como em análise exploratória, as wavelets não ortogonais são úteis; e no caso que seja necessária a análise de mudanças de amplitude e fase, as wavelets complexas são mais interessantes, como será visto adiante. A seguir serão mostradas algumas wavelets de forma breve, sendo a wavelet harmônica mostrada mais detalhadamente em seções posteriores.

A primeira a presentada é a Wavelet de Haar, esta é o tipo mais simples de wavelet, sendo sua wavelet mãe definida por:

$$
\psi(t)=\mid \begin{gathered}
1, \text { se } 0 \leq t \leq 1 / 2 \\
-1, \text { se } 1 / 2 \leq t \leq 1 \\
0, \text { se } t<0 \text { ou } t>1
\end{gathered}
$$

E seu gráfico é mostrado na Figura 4.3:

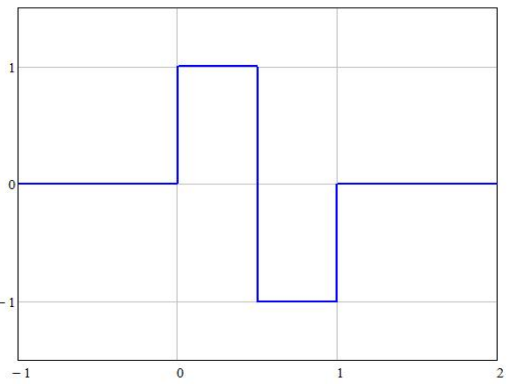

Figura 4.3. Wavelet de Haar. 
Uma wavelet bastante conhecida é a Wavelet de Morlet, esta pode também ser chamada de gaussiana modulada, além disto, esta é uma função complexa definida por:

$\psi(t)=e^{i \omega t} e^{-t^{2} / 2}$

Onde a partir da relação (3.31) pode ser decomposta em uma parte real e outra imaginária:

$\psi(t)=(\cos (\omega t)+i \operatorname{sen}(\omega t)) e^{-t^{2} / 2}$

Com seus gráficos reais e imaginários sendo representados abaixo (Figura 4.4(a) e 4.4(b)):
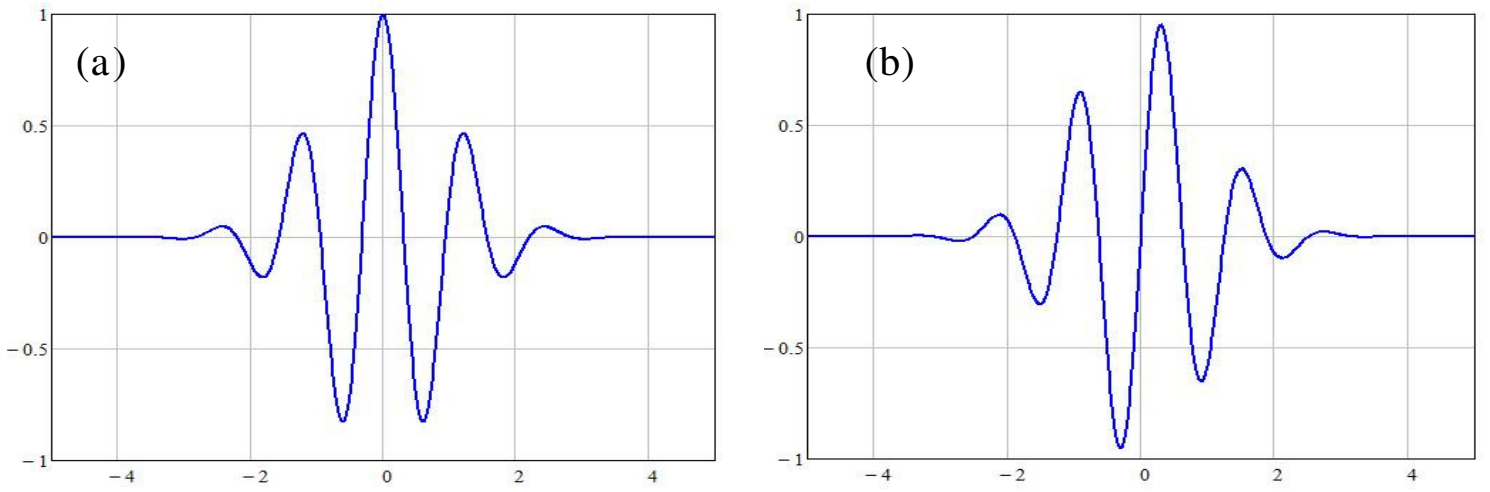

Figura 4.4. Wavelet de Morlet: (a) parcela real; (b) parcela imaginária.

A Wavelet chapéu mexicano é denominada assim devido a sua forma, onde é definida pela função:

$\psi(t)=\left(1-t^{2}\right) e^{-t^{2} / 2}$

Sendo seu gráfico (Figura 4.5) representado por:

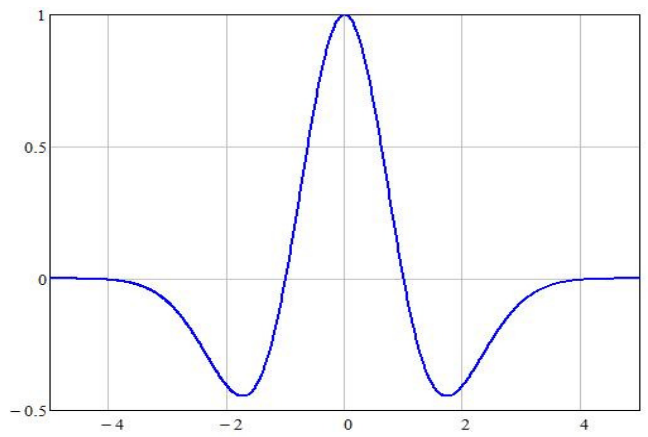

Figura 4.5. Wavelet chapéu mexicano. 
Por ultimo, tem-se a Wavelet de Daubechies, que podem ser também chamadas de wavelets ortonomais de suporte compacto, e de acordo com Campanha (2004), sua grande importância foi tornar a análise discreta das wavelets computacionalmente mais simples. Estas são obtidas através da função de escala, possuindo o formato:

$\phi^{(\mathrm{n})}(\mathrm{t})=\sum_{\mathrm{k}=0}^{\mathrm{N}-1} l_{\mathrm{k}} \phi^{(\mathrm{n}-1)}(2 \mathrm{t}-\mathrm{k})$

Onde (4.29) é obtida através de um processo interativo aplicado $\mathrm{n}$ vezes, até uma aproximação aceitável para o que se deseja. Esta função de escala tem como formato inicial:

$\phi^{(0)}(\mathrm{t})=\mid \begin{array}{ll}1, & \text { se } 0 \leq \mathrm{t} \leq 1 \\ 0, & \text { se } \mathrm{t}<0 \text { ou } \mathrm{t}>1\end{array}$

Segundo Newland (1993), a função (4.30) é chamada de função caixa (box function). A partir da função de escala obtida em (4.29), pode-se através desta, obter a wavelet mãe:

$\psi(\mathrm{t})=\sum_{\mathrm{k}=0}^{\mathrm{N}-1} h_{\mathrm{k}} \phi^{(\mathrm{n})}(2 \mathrm{t}-\mathrm{k})$

Onde:

$h_{\mathrm{k}}=(-1)^{\mathrm{k}} l_{\mathrm{N}-\mathrm{k}}$

De acordo com Morettin (1999), $l_{\mathrm{k}}$ e $h_{\mathrm{k}}$ são coeficientes de filtros passa-baixo (lowpass) e passa-alto (high-pass) respectivamente, onde estes nomes são explicados devido ao fato que o primeiro pertence à função de escala, a qual gera as freqüências menores do sinal, e o segundo, a wavelet mãe, onde são geradas as frequiências maiores.

Para a geração de wavelets com bom funcionamento, os coeficientes devem ser escolhidos com cuidado, segundo Newland (1993), há três condições necessárias.

A primeira é a condição de conservação da área, onde mostra que o somatório de todos os coeficientes $l_{\mathrm{k}}$ devem ter um valor igual a 2 , com isto:

$\sum_{\mathrm{k}=0}^{\mathrm{N}-1} l_{\mathrm{k}}=2$ 
A segunda condição é chamada de condição de precisão, esta condição assegura que a expansão do comprimento do sinal com um número finito de wavelets permita reproduzir o sinal da forma mais precisa possível, esta condição gera N/2-1 equações linearmente independentes na forma:

$\sum_{\mathrm{k}=0}^{\mathrm{N}-1}(-1)^{\mathrm{k}} \mathrm{k}^{\mathrm{r}} l_{\mathrm{k}}=0 \quad$ para $\quad \mathrm{r}=1,2, \ldots, \mathrm{N} / 2-1$

Onde (4.34), segundo Newland, vem da condição de precisão de Strang (Strang's accuracy condition) que mostra que a transformada de Fourier da função de escala deve ser periodicamente nula para garantir uma representação mais verdadeira do sinal. A terceira e ultima é a condição de ortogonalidade, esta condição assegura que a função de escala e a wavelet gerada a partir dela são ortogonais entre si, sem esta, a DWT não funciona, esta condição gera N/2 equações não-lineares independentes na forma:

$$
\begin{aligned}
& \sum_{\mathrm{k}=0}^{\mathrm{N}-1} l_{\mathrm{k}}^{2}=2 \\
& \sum_{\mathrm{k}=0}^{\mathrm{N}-1-2 \mathrm{r}} l_{\mathrm{k}} l_{\mathrm{k}+2 \mathrm{r}}=0 \quad \text { para } \quad \mathrm{r}=1,2, \ldots, \mathrm{N} / 2-1
\end{aligned}
$$

Com a união das três condições chega-se a $\mathrm{N}$ equações e $\mathrm{N}$ incógnitas, que são os coeficientes $l_{\mathrm{k}}$, onde se forma um sistema não-linear possível determinado, sendo que sua solução fornece os coeficientes utilizados na função de escala, e consequentemente os coeficientes $h_{\mathrm{k}}$ da wavelet. Observando o número de equações geradas por cada condição, percebe-se que a quantidade de coeficientes da função de escala precisam ser pares.

A família de wavelet de Daubechies são denotadas por $\mathrm{dbN}$, sendo " $\mathrm{N}$ " a ordem da wavelet e "db" do fato de terem sido criadas por Daubechies, estas wavelets possuem um domínio não-nulo bem definido no tempo de N-1. Os gráficos da função de escala e da wavelet de Daubechies possuem natureza fractal, sendo comuns descontinuidades, para a obtenção de funções mais suaves, é necessário incluir mais termos (e consequentemente mais coeficientes) na função de escala (Figura 4.6). 

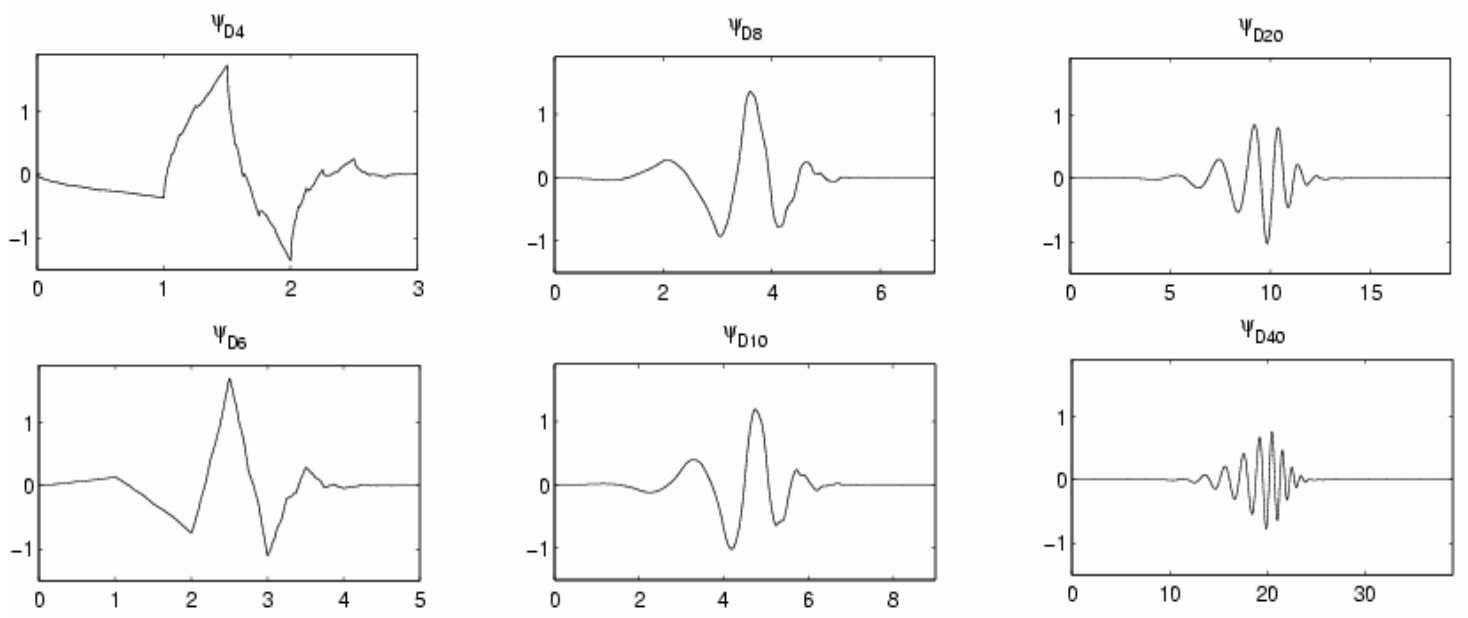

Figura 4.6. Parte da família de wavelets de Daubechies.

O algoritmo da DWT para estas wavelets foi criado por Mallat, sendo chamado de algoritmo piramidal de Mallat (Mallat's pyramid algorithm). Este algoritmo é dividido em dois estágios, sendo um para o cálculo da DWT e outro para o cálculo da Transformada inversa de Wavelet ou IDWT (Inverse Discret Wavelet Transform).

As análises em várias escalas podem ser obtidas utilizando-se um banco de filtros composto por filtros passa-baixa de decomposição L, gerados a partir dos coeficientes $l_{\mathrm{k}}$ e filtros passa-alta de decomposição $\mathrm{H}$, gerados a partir dos coeficientes $h_{\mathrm{k}}$, juntamente com os seus filtros de reconstrução associados L' e H', também gerados a partir de $l_{\mathrm{k}}$ e $h_{\mathrm{k}}$, estes, formam um sistema chamado de filtro de quadratura espelhada ou QMF (Quadrature Mirror Filters).

A partir de um sinal $\mathrm{x}(\mathrm{t})$, dois conjuntos de coeficientes podem ser produzidos a partir da DWT, que são os coeficientes de aproximação $u_{k}$ e coeficientes de detalhe $w_{j, k}$. Estes conjuntos são obtidos através da superposição de $\mathrm{x}(\mathrm{t})$ com o filtro passa-baixa $\mathrm{L}$ para as aproximações, e com o filtro passa-alta $\mathrm{H}$ para os detalhes, seguido pelo desprezo de uma a cada duas saídas do filtro.

Neste estágio, conhecido como decomposição, ou análise, se determina o conjunto de coeficientes $\mathrm{u}_{\mathrm{k}} \mathrm{e} \mathrm{w}_{\mathrm{j}, \mathrm{k}}$. Em uma decomposição de múltiplos níveis, é necessário que o sinal $\mathrm{x}(\mathrm{t})$ possua numero de pontos equivalentes a uma potencia de 2 , para $\mathrm{o}$ funcionamento do algoritmo. 
Fazendo o caminho inverso, parte-se de $\mathrm{u}_{\mathrm{k}}$ e $\mathrm{w}_{\mathrm{j}, \mathrm{k}}$, a IDWT reconstrói o sinal x(t) através da inserção de zeros entre os filtros de reconstrução $\mathrm{H}^{\prime}$, com isto, se aplica à superposição do sinal resultante gerado a partir dos filtros de reconstrução L' e H'.

Este é o estágio de reconstrução, ou síntese. Quando ocorre um processo de decomposição de múltiplos níveis, o sinal $\mathrm{x}(\mathrm{t})$ pode ser conseguido por aproximações sucessivas que são decompostas de modo que o sinal seja dividido em vários componentes de resolução mais baixa. 


\subsection{WAVELET HARMÔNICA}

Para a geração de carregamentos sintéticos, os quais se desejam induzir um comportamento aleatório de fase, uma wavelet complexa pode ser a mais adequada, como a de Morlet, mas esta ultima possui sua desvantagem no campo numérico. Uma wavelet complexa com algoritmos que geram menor esforço computacional foi criada por Newland (1993), sendo chamado por este autor de wavelet harmônica, que nasceu a partir da análise da wavelet de Daubechies.

Foi analisado que quanto maior o número de coeficientes $\mathrm{N}$ a wavelet de Daubechies possui, ocorre o aumento progressivo da extensão de seu domínio e sua transformada de Fourier adquire um espectro cada vez mais compacto, alcançando um formato similar a uma "caixa" ou retângulo, quando o número de coeficientes $\mathrm{N}$ for suficientemente grande (Figura 4.7(a), 4.7(b) e 4.7(c)).
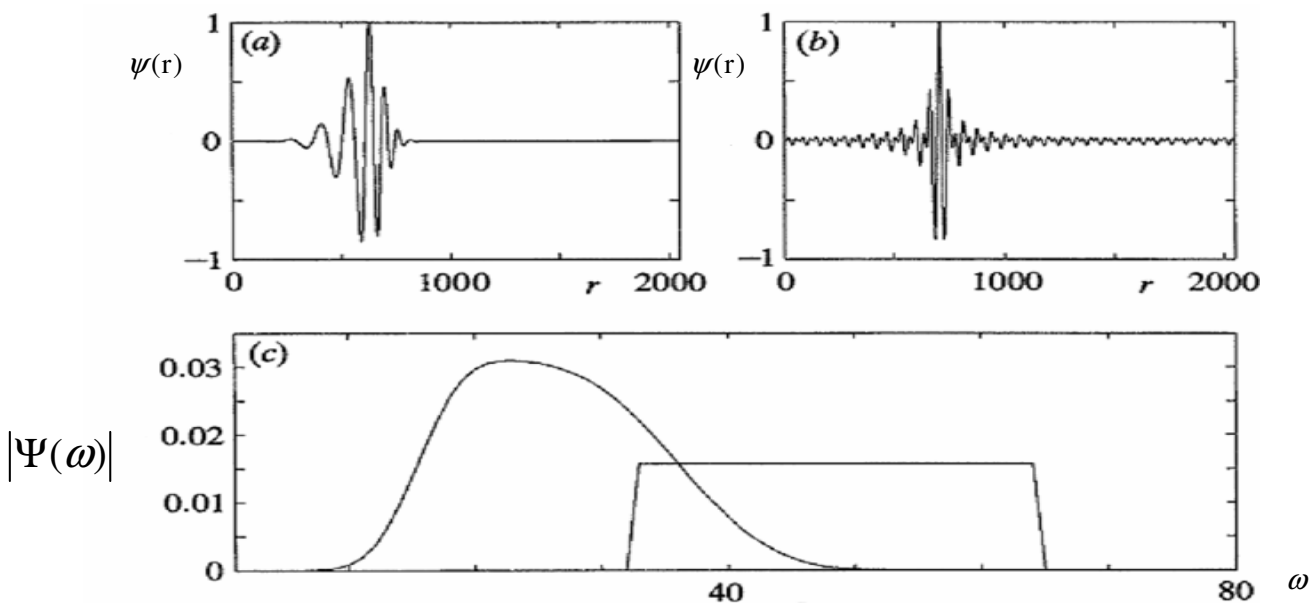

Figura 4.7. Comparação entre as DFT: (a) Wavelet db20; (b) Wavelet harmônica; (c) DFT das duas, onde a db20 esta localizada a esquerda (Imagem modificada a partir de Newland (1993)).

Este fato levou a procura de uma wavelet na qual o espectro devido à transformada de Fourier fosse exatamente como uma caixa, e que sua magnitude fosse diferente de zero apenas em um intervalo especificado de freqüências, devido a isto, Newland (1993) definiu dois espectros:

$$
\Psi^{\mathrm{e}}(\omega)=\mid \begin{array}{lll}
1 / 4 \pi & \text { se } & 2 \pi \leq \omega<4 \pi \\
1 / 4 \pi & \text { se } & -2 \pi \leq \omega<-4 \pi \\
0 & \text { se } & -2 \pi<\omega<2 \pi \text { ou } \omega \geq 4 \pi \text { ou } \omega \leq-4 \pi
\end{array}
$$


$\Psi^{\circ}(\omega)=\mid \begin{array}{lll}i / 4 \pi & \text { se } & 2 \pi \leq \omega<4 \pi \\ -i / 4 \pi & \text { se } & -2 \pi \leq \omega<-4 \pi \\ 0 & \text { se } & -2 \pi<\omega<2 \pi \text { ou } \omega \geq 4 \pi \text { ou } \omega \leq-4 \pi\end{array}$

Onde $\omega$ é a freqüência angular, nota-se de acordo com (3.8) que o valor de T é equivalente a um valor unitário, esta consideração na resolução destas funções não restringirá seu uso para outros valores de T. Então, dados os espectros, foi definida uma relação entre eles:

$\Psi(\omega)=\Psi^{\mathrm{e}}(\omega)+i \Psi^{\mathrm{o}}(\omega)$

O que resulta em:

$\Psi(\omega)=\mid \begin{array}{lll}1 / 2 \pi & \text { se } & 2 \pi \leq \omega<4 \pi \\ 0 & \text { se } & \omega<2 \pi \text { ou } \omega \geq 4 \pi\end{array}$

Aplicando a Transformada inversa de Fourier em (4.40), chega-se:

$\psi(\mathrm{t})=\left(\mathrm{e}^{i 4 \pi \mathrm{t}}-\mathrm{e}^{i 2 \pi \mathrm{t}}\right) / i 2 \pi \mathrm{t}$

Onde (4.41) é a wavelet harmônica mãe, esta tem as representações gráficas de acordo com as Figuras 4.8(a) e 4.8(b):
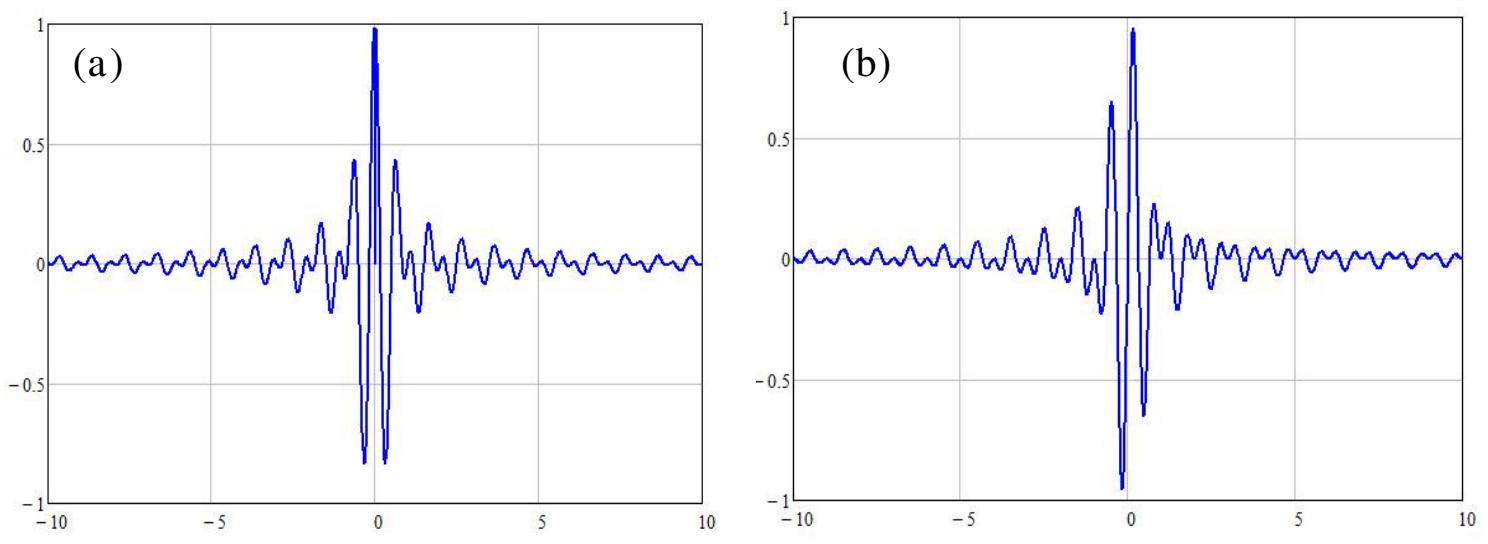

Figura 4.8. Wavelet harmônica: (a) parcela real; (b) parcela imaginária.

A função (4.41) também pode ser obtida pela Transformada inversa de Fourier de (4.37) e (4.38), a partir disto, se aplica a relação:

$\psi(\omega)=\psi^{\mathrm{e}}(\omega)+i \psi^{\mathrm{o}}(\omega)$ 
Para a geração das wavelets harmônicas filhas, com escalas binárias e translações diádicas, é necessário utilizar o seguinte espectro de freqüência:

$$
\Psi_{j, \mathrm{k}}(\omega)=\mid \begin{array}{lll}
(1 / 2 \pi) 2^{-\mathrm{j}} \mathrm{e}^{-i \omega \mathrm{k} / 2^{\mathrm{j}}} & \text { se } & 2 \pi 2^{\mathrm{j}} \leq \omega<4 \pi 2^{\mathrm{j}} \\
0 & \text { se } & \omega<2 \pi 2^{\mathrm{j}} \text { ou } \omega \geq 4 \pi 2^{\mathrm{j}}
\end{array}
$$

Com a aplicação da Transformada inversa de Fourier em (4.43), tem-se:

$\psi\left(2^{\mathrm{j}} \mathrm{t}-\mathrm{k}\right)=\left(\mathrm{e}^{i 4 \pi\left(2^{\mathrm{j}} \mathrm{t}-\mathrm{k}\right)}-\mathrm{e}^{i 2 \pi\left(2^{\mathrm{j}} \mathrm{t}-\mathrm{k}\right)}\right) / i 2 \pi\left(2^{\mathrm{j}} \mathrm{t}-\mathrm{k}\right)$

Utilizando (4.44) na relação (4.12) :

$\psi_{\mathrm{j}, \mathrm{k}}(\mathrm{t})=2^{\mathrm{j} / 2}\left(\mathrm{e}^{i 4 \pi\left(2^{\mathrm{j}} \mathrm{t}-\mathrm{k}\right)}-\mathrm{e}^{i 2 \pi\left(2^{\mathrm{j}} \mathrm{t}-\mathrm{k}\right)}\right) / i 2 \pi\left(2^{\mathrm{j}} \mathrm{t}-\mathrm{k}\right)$

A função (4.45) é a wavelet harmônica filha. Como toda wavelet, estas possuem média nula, por isto, para a geração ou reconstrução de sinais com média diferente de zero, ou de comportamento não-estacionário, é necessário o uso de uma função de escala, esta, é oriunda das funções (4.37) e (4.38), então:

$$
\begin{aligned}
& \Phi^{\mathrm{e}}(\omega)=\mid \begin{array}{llc}
1 / 4 \pi & \text { se } & -2 \pi \leq \omega<2 \pi \\
0 & \text { se } & \omega \geq 4 \pi \text { ou } \omega \leq-4 \pi
\end{array} \\
& \Phi^{\mathrm{o}}(\omega)=\mid \begin{array}{llc}
i / 4 \pi & \text { se } & 0 \leq \omega<2 \pi \\
-i / 4 \pi & \text { se } & -2 \pi \leq \omega<0 \\
0 & \text { se } & \omega \geq 2 \pi \text { ou } \omega \leq-2 \pi
\end{array}
\end{aligned}
$$

Assim como as wavelets, é definida a relação:

$$
\Phi(\omega)=\Phi^{\mathrm{e}}(\omega)+i \Phi^{\mathrm{o}}(\omega)
$$

O que resulta em:

$$
\Phi(\omega)=\mid \begin{array}{lll}
1 / 2 \pi & \text { se } & 0 \leq \omega<2 \pi \\
0 & \text { se } & \omega<0 \text { ou } \omega \geq 2 \pi
\end{array}
$$

Aplicando a Transformada inversa de Fourier em (4.49), chega-se:

$\phi(\mathrm{t})=\left(\mathrm{e}^{i 2 \pi \mathrm{t}}-1\right) / i 2 \pi \mathrm{t}$

Onde (4.50) é a função de escala ou wavelet harmônica pai, esta tem as representações gráficas de acordo com as Figuras 4.9(a) e 4.9(b): 

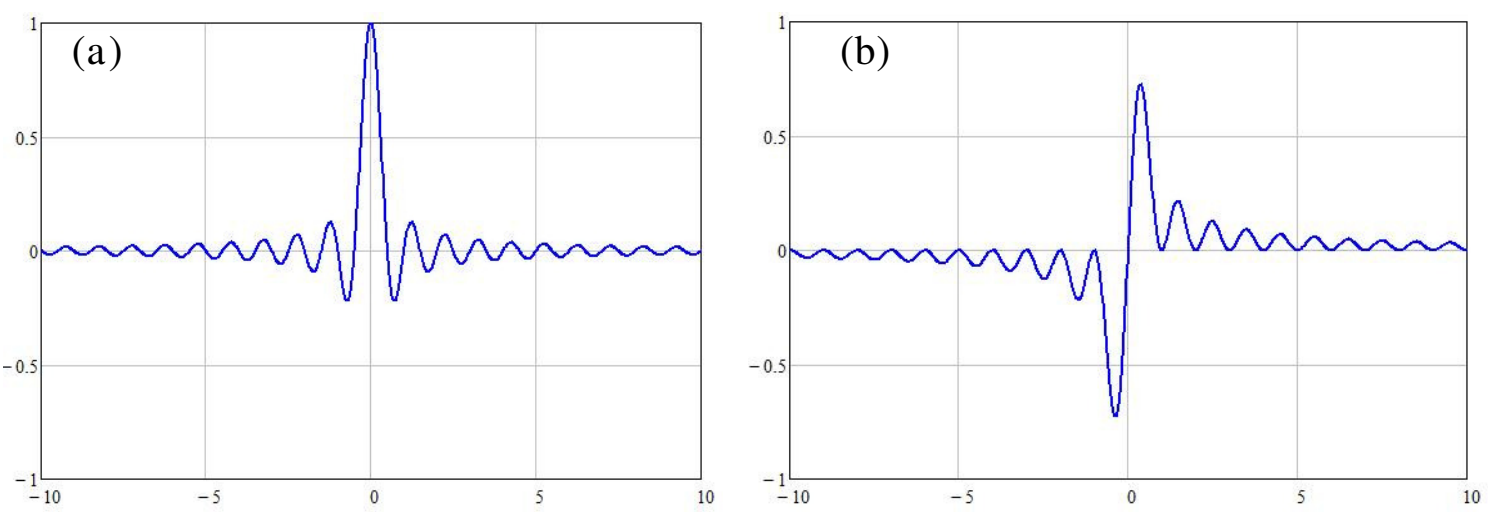

Figura 4.9. Função de escala: (a) parcela real; (b) parcela imaginária.

A função (4.50) também pode ser obtida pela Transformada inversa de Fourier de (4.46) e (4.47), com o uso da mesma relação de (4.42):

$\phi(\omega)=\phi^{\mathrm{e}}(\omega)+i \phi^{\mathrm{o}}(\omega)$

Baseado em (4.43), mas com a não consideração de variações na escala para este tipo de função:

$\Phi_{\mathrm{k}}(\omega)=\begin{array}{lll}(1 / 2 \pi) \mathrm{e}^{-i \omega \mathrm{k}} & \text { se } & 0 \leq \omega<2 \pi \\ 0 & \text { se } & \omega<0 \text { ou } \omega \geq 2 \pi\end{array}$

Com a aplicação da Transformada inversa de Fourier em (4.52), tem-se:

$\phi(\mathrm{t}-\mathrm{k})=\left(\mathrm{e}^{i 2 \pi(\mathrm{t}-\mathrm{k})}-1\right) / i 2 \pi(\mathrm{t}-\mathrm{k})$

Utilizando (4.53) na relação (4.20):

$\phi_{\mathrm{k}}(\mathrm{t})=\left(\mathrm{e}^{i 2 \pi(\mathrm{t}-\mathrm{k})}-1\right) / i 2 \pi(\mathrm{t}-\mathrm{k})$

Para as wavelets harmônicas e suas funções de escala, há duas wavelets e duas funções de escala para cada par de $\mathrm{j}$ e $\mathrm{k}$, isto ocorre devido a existência de uma parcela par obtida a partir de (4.37) e (4.46) e uma impar, obtida a partir de (4.38) e (4.47). Estas parcelas também geram wavelets e funções de escalas se seguirem às relações:

$$
\begin{aligned}
& \bar{\psi}(\omega)=\psi^{\mathrm{e}}(\omega)-i \psi^{\mathrm{o}}(\omega) \\
& \bar{\phi}(\omega)=\phi^{\mathrm{e}}(\omega)-i \phi^{\mathrm{o}}(\omega)
\end{aligned}
$$


Desenvolvendo (4.55) e (4.56), tem-se:

$$
\begin{aligned}
& \bar{\psi}(\mathrm{t})=\left(\mathrm{e}^{-i 2 \pi \mathrm{t}}-\mathrm{e}^{-i 4 \pi \mathrm{t}}\right) / i 2 \pi \mathrm{t} \\
& \bar{\phi}(\mathrm{t})=\left(1-\mathrm{e}^{-i 2 \pi \mathrm{t}}\right) / i 2 \pi \mathrm{t}
\end{aligned}
$$

A wavelet harmônica (4.57) e a função de escala (4.58) são os conjugados complexos da wavelet harmônica (4.41) e da função de escala (4.50) respectivamente. As wavelets e funções de escala filhas delas podem ser obtidas a partir de (4.57) e (4.58) através do uso de (4.12) e (4.20):

$$
\begin{aligned}
& \bar{\psi}_{\mathrm{j}, \mathrm{k}}(\mathrm{t})=2^{\mathrm{j} / 2}\left(\mathrm{e}^{-i 2 \pi\left(2^{\mathrm{j}} \mathrm{t}-\mathrm{k}\right)}-\mathrm{e}^{-i 4 \pi\left(2^{\mathrm{j}} \mathrm{t}-\mathrm{k}\right)}\right) / i 2 \pi\left(2^{\mathrm{j}} \mathrm{t}-\mathrm{k}\right) \\
& \bar{\phi}_{\mathrm{k}}(\mathrm{t})=\left(1-\mathrm{e}^{-i 2 \pi(\mathrm{t}-\mathrm{k})}\right) / i 2 \pi(\mathrm{t}-\mathrm{k})
\end{aligned}
$$

A partir de todos os espectros de freqüência apresentados, pode-se observar a ortogonalidade entre eles na Figura 4.10:

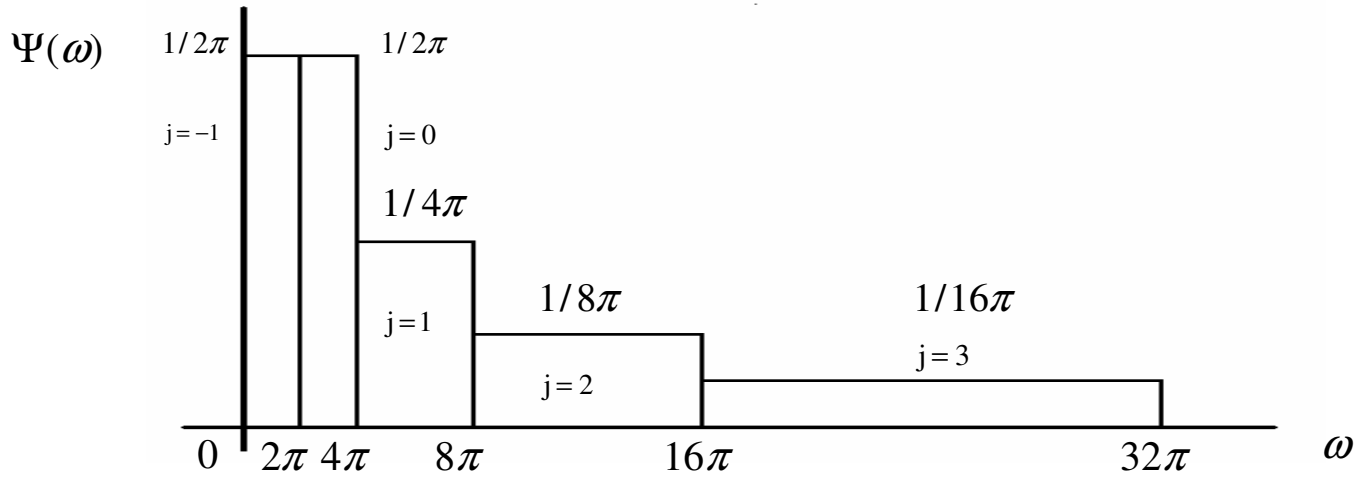

Figura 4.10. Espectro de freqüência obtido através de Transformadas de Fourier de wavelets harmônicas de diferentes níveis.

Assim como as wavelets de Daubechies, as wavelets harmônicas e suas funções de escala constituem uma família de funções ortogonais, onde a ortogonalidade vem de uma relação entre o produto interno de duas wavelets harmônicas ou funções de escala quaisquer, com o produto interno de suas respectivas transformadas (Figura 4.10).

Como o produto interno de suas transformadas, representadas pelos espectros de freqüência, é nulo, este valor se repete para o produto interno das wavelets harmônicas e de suas funções de escala, para maiores detalhes, a relação descrita pode ser provada em Newland (1993). Devido a ortogonalidade, as relações (4.13) e (4.21) são validas, alem destas, as seguintes relações também tem validade: 


$$
\begin{array}{lll}
\left\langle\psi_{\mathrm{j}, \mathrm{k}_{1}}, \phi_{\mathrm{k}_{2}}\right\rangle=\left\langle\bar{\Psi}_{\mathrm{j}, \mathrm{k}_{1}}, \bar{\phi}_{\mathrm{k}_{2}}\right\rangle=0 & \text { para } & \mathrm{j} \geq 0 \\
\left\langle\psi_{\mathrm{j}, \mathrm{k}_{1}}, \bar{\phi}_{\mathrm{k}_{2}}\right\rangle=\left\langle\bar{\Psi}_{\mathrm{j}, \mathrm{k}_{1}}, \phi_{\mathrm{k}_{2}}\right\rangle=0 & \text { para } & \mathrm{j} \geq 0 \\
\left\langle\bar{\Psi}_{\mathrm{j}_{1}, \mathrm{k}_{1}}, \psi_{\mathrm{j}_{2}, \mathrm{k}_{2}}\right\rangle=\left\langle\psi_{\mathrm{j}_{1}, \mathrm{k}_{1}}, \bar{\Psi}_{\mathrm{j}_{2}, \mathrm{k}_{2}}\right\rangle=0 & \text { para } & \mathrm{j}_{1} \neq \mathrm{j}_{2} \text { e } \mathrm{k}_{1} \neq \mathrm{k}_{2} \\
\left\langle\bar{\phi}_{\mathrm{k}_{1}}, \phi_{\mathrm{k}_{2}}\right\rangle=\left\langle\phi_{\mathrm{k}_{1}}, \bar{\phi}_{\mathrm{k}_{2}}\right\rangle=0 & \text { para } & \mathrm{k}_{1} \neq \mathrm{k}_{2} \\
\left\langle\Psi_{\mathrm{j}_{1}, \mathrm{k}_{1}}, \psi_{\mathrm{j}_{2}, \mathrm{k}_{2}}\right\rangle=\left\langle\bar{\Psi}_{\mathrm{j}_{1}, \mathrm{k}_{1}}, \bar{\Psi}_{\mathrm{j}_{2}, \mathrm{k}_{2}}\right\rangle=0 & \text { para } & \mathrm{j}_{1} \neq \mathrm{j}_{2} \mathrm{e}_{1} \neq \mathrm{k}_{2} \\
\left\langle\phi_{\mathrm{k}_{1}}, \phi_{\mathrm{k}_{2}}\right\rangle=\left\langle\bar{\phi}_{\mathrm{k}_{1}}, \bar{\phi}_{\mathrm{k}_{2}}\right\rangle=0 & \text { para } & \mathrm{k}_{1} \neq \mathrm{k}_{2}
\end{array}
$$

E a ortonormalidade das wavelets harmônicas e das funções de escala podem ser verificadas através dos seguintes produtos internos:

$$
\begin{array}{lll}
\left\langle\bar{\Psi}_{\mathrm{j}_{1}, \mathrm{k}_{1}}, \psi_{\mathrm{j}_{2}, \mathrm{k}_{2}}\right\rangle=\left\langle\psi_{\mathrm{j}_{1}, \mathrm{k}_{1}}, \bar{\Psi}_{\mathrm{j}_{2}, \mathrm{k}_{2}}\right\rangle=1 & \text { para } & \mathrm{j}_{1}=\mathrm{j}_{2} \text { e } \mathrm{k}_{1}=\mathrm{k}_{2} \\
\left\langle\bar{\phi}_{\mathrm{k}_{1}}, \phi_{\mathrm{k}_{2}}\right\rangle=\left\langle\phi_{\mathrm{k}_{1}}, \bar{\phi}_{\mathrm{k}_{2}}\right\rangle=1 & \text { para } & \mathrm{k}_{1}=\mathrm{k}_{2}
\end{array}
$$

Onde (4.67) e (4.68) são equivalentes as equações:

$$
\begin{aligned}
& \int_{-\infty}^{+\infty}\left|\psi_{\mathrm{j}, \mathrm{k}}(\mathrm{t})\right|^{2} \mathrm{dt}=1 \\
& \int_{-\infty}^{+\infty}\left|\phi_{\mathrm{k}}(\mathrm{t})\right|^{2} \mathrm{dt}=1
\end{aligned}
$$

Estas últimas relações são importantes para o entendimento da reconstrução ou geração de um sinal $\mathrm{x}(\mathrm{t})$, seja ele real ou complexo, em termos das wavelets harmônicas. Para a reconstrução ou geração de um sinal, tendo as funções base, é necessário saber os valores das ponderações destas funções, para isto, usa-se a Transformada discreta da wavelet harmônica ou DHWT (Discrete Harmonic Wavelet Transform), assim como estas wavelets, existem duas DHWT para cada par de $\mathrm{j}$ e $\mathrm{k}$, devido a isto, Newland (1993) definiu os coeficientes para as wavelets harmônicas como:

$$
\begin{aligned}
& w_{j, k}=2^{j / 2} \int_{-\infty}^{+\infty} x(t) \bar{\psi}_{j, k} d t \\
& \tilde{w}_{j, k}=2^{j / 2} \int_{-\infty}^{+\infty} x(t) \psi_{j, k} d t
\end{aligned}
$$


E para as funções de escala da wavelet harmônica na forma:

$$
\begin{aligned}
& \mathrm{u}_{\mathrm{k}}=\int_{-\infty}^{+\infty} \mathrm{x}(\mathrm{t}) \bar{\phi}_{\mathrm{k}} \mathrm{dt} \\
& \tilde{\mathrm{u}}_{\mathrm{k}}=\int_{-\infty}^{+\infty} \mathrm{x}(\mathrm{t}) \phi_{\mathrm{k}} \mathrm{dt}
\end{aligned}
$$

Os sinais que podem ser representados por esta wavelet obedecem a condição (4.1), a partir disto, a transformada inversa discreta da wavelet harmônica ou IDHWT (Inverse Discret Harmonic Wavelet Transform) é expressa por:

$$
\mathrm{x}(\mathrm{t})=\left[\begin{array}{l}
\sum_{\mathrm{k}=-\infty}^{+\infty}\left(\mathrm{u}_{\mathrm{k}} \phi(\mathrm{t}-\mathrm{k})+\tilde{\mathrm{u}}_{\mathrm{k}} \bar{\phi}(\mathrm{t}-\mathrm{k})\right)+ \\
\sum_{\mathrm{j}=0}^{+\infty} \sum_{\mathrm{k}=-\infty}^{+\infty}\left(\mathrm{w}_{\mathrm{j}, \mathrm{k}} \psi\left(2^{\mathrm{j}} \mathrm{t}-\mathrm{k}\right)+\tilde{\mathrm{w}}_{\mathrm{j}, \mathrm{k}} \bar{\psi}\left(2^{\mathrm{j}} \mathrm{t}-\mathrm{k}\right)\right)
\end{array}\right]
$$

Se $x(t)$ for complexo, considera-se $\tilde{\mathrm{w}}_{\mathrm{j}, \mathrm{k}}$ e $\tilde{\mathrm{u}}_{\mathrm{k}}$ como amplitudes diferentes de $\mathrm{w}_{\mathrm{j}, \mathrm{k}}$ e $\mathrm{u}_{\mathrm{k}}$. Mas quando x(t) for real, $\tilde{\mathrm{w}}_{\mathrm{j}, \mathrm{k}}$ e $\tilde{\mathrm{u}}_{\mathrm{k}}$ são os conjugados complexos de $\mathrm{w}_{\mathrm{j}, \mathrm{k}}$ e $\mathrm{u}_{\mathrm{k}}$, então $\tilde{\mathrm{w}}_{\mathrm{j}, \mathrm{k}}=\overline{\mathrm{w}}_{\mathrm{j}, \mathrm{k}}$ e $\tilde{\mathrm{u}}_{\mathrm{k}}=\overline{\mathrm{u}}_{\mathrm{k}}$, com isto, IDHWT pode ser expressa na forma:

$$
\mathrm{x}(\mathrm{t})=\left[\begin{array}{l}
\sum_{\mathrm{k}=-\infty}^{+\infty}\left(\mathrm{u}_{\mathrm{k}} \phi(\mathrm{t}-\mathrm{k})+\overline{\mathrm{u}}_{\mathrm{k}} \bar{\phi}(\mathrm{t}-\mathrm{k})\right)+ \\
\sum_{\mathrm{j}=0}^{+\infty} \sum_{\mathrm{k}=-\infty}^{+\infty}\left(\mathrm{w}_{\mathrm{j}, \mathrm{k}} \psi\left(2^{\mathrm{j}} \mathrm{t}-\mathrm{k}\right)+\overline{\mathrm{w}}_{\mathrm{j}, \mathrm{k}} \bar{\psi}\left(2^{\mathrm{j}} \mathrm{t}-\mathrm{k}\right)\right)
\end{array}\right]
$$




\subsection{ANÁLISE NUMÉRICA DA WAVELET HARMÔNICA}

Abordada a teoria básica sobre as wavelets harmônicas, pode-se perceber que existem algumas desvantagem e vantagens em relação às wavelets de Daubechies. As desvantagens residem nos fatos que as wavelets harmômicas não são bem definidas no domínio do tempo, decaindo em uma proporção de 1/t e que seus coeficientes são geralmente complexos, havendo dois coeficientes para cada par $\mathrm{j}, \mathrm{k}$, como mostrado anteriormente.

Quanto a suas vantagens, as wavelets harmônicas podem ser descritas por funções analíticas simples, devido ao principio da incerteza, são bem definidas no domínio da freqüência em seu espectro gerado pela transformada de Fourier, em conseqüência desta característica, são computacionalmente mais simples e mais rápidas que as wavelets de Daubechies, pois podem usar em seu algoritmo a FFT, como será visto adiante.

Para gerar um algoritmo que processe os coeficientes da wavelet harmônicas, primeiro, é necessária a consideração da equação (4.43), aplicando a transformada inversa de Fourier nesta, usa-se a relação (4.12):

$$
\begin{aligned}
& \psi_{\mathrm{j}, \mathrm{k}}=2^{\mathrm{j} / 2} \int_{2 \pi 2^{\mathrm{j}}}^{4 \pi 2^{\mathrm{j}}} \Psi_{\mathrm{j}, \mathrm{k}}(\omega) \mathrm{e}^{\mathrm{i} \omega \mathrm{t}} \mathrm{d} \omega \\
& \bar{\psi}_{\mathrm{j}, \mathrm{k}}=2^{\mathrm{j} / 2} \int_{2 \pi 2^{\mathrm{j}}}^{4 \pi 2^{\mathrm{j}}} \Psi_{\mathrm{j}, \mathrm{k}}(-\omega) \mathrm{e}^{-\mathrm{i} \omega \mathrm{t}} \mathrm{d} \omega
\end{aligned}
$$

Aplicando a relação (4.77) em (4.72), assim como (4.78) em (4.71), tem-se:

$$
\begin{aligned}
& \mathrm{w}_{\mathrm{j}, \mathrm{k}}=2^{\mathrm{j}} \int_{-\infty}^{+\infty} \mathrm{x}(\mathrm{t}) \int_{2 \pi 2^{\mathrm{j}}}^{4 \pi 2^{\mathrm{j}}} \Psi_{\mathrm{j}, \mathrm{k}}(-\omega) \mathrm{e}^{-\mathrm{i} \omega \mathrm{t}} \mathrm{d} \omega \mathrm{dt} \\
& \tilde{\mathrm{w}}_{\mathrm{j}, \mathrm{k}}=2^{\mathrm{j}} \int_{-\infty}^{+\infty} \mathrm{x}(\mathrm{t}) \int_{2 \pi 2^{\mathrm{j}}}^{4 \pi 2^{\mathrm{j}}} \Psi_{\mathrm{j}, \mathrm{k}}(\omega) \mathrm{e}^{\mathrm{i} \omega \mathrm{t}} \mathrm{d} \omega \mathrm{dt}
\end{aligned}
$$

Abrindo (4.43) e realizando alguma manipulação algébrica, chega-se:

$$
\begin{aligned}
& \mathrm{w}_{\mathrm{j}, \mathrm{k}}=\int_{2 \pi 2^{\mathrm{j}}}^{4 \pi 2^{\mathrm{j}}}\left(\frac{1}{2 \pi} \int_{-\infty}^{+\infty} \mathrm{x}(\mathrm{t}) \mathrm{e}^{-i \omega \mathrm{t}} \mathrm{dt}\right) \mathrm{e}^{i \omega \mathrm{k} / 2^{\mathrm{j}}} \mathrm{d} \omega \\
& \tilde{\mathrm{w}}_{\mathrm{j}, \mathrm{k}}=\int_{2 \pi 2^{\mathrm{j}}}^{4 \pi 2^{\mathrm{j}}}\left(\frac{1}{2 \pi} \int_{-\infty}^{+\infty} \mathrm{x}(\mathrm{t}) \mathrm{e}^{i \omega \mathrm{t}} \mathrm{dt}\right) \mathrm{e}^{-i \omega \mathrm{k} / 2^{\mathrm{j}}} \mathrm{d} \omega
\end{aligned}
$$


Percebe-se que em (4.81) e (4.82), as partes entre parênteses são as Transformada de Fourier como apresentado em (3.34), com isto:

$$
\begin{aligned}
& \mathrm{w}_{\mathrm{j}, \mathrm{k}}=\int_{2 \pi 2^{\mathrm{j}}}^{4 \pi 2^{\mathrm{j}}} \mathrm{X}(\omega) \mathrm{e}^{i \omega \mathrm{k} / 2^{\mathrm{j}}} \mathrm{d} \omega \\
& \tilde{\mathrm{w}}_{\mathrm{j}, \mathrm{k}}=\int_{2 \pi 2^{\mathrm{j}}}^{4 \pi 2^{\mathrm{j}}} \mathrm{X}(-\omega) \mathrm{e}^{-i \omega \mathrm{k} / 2^{\mathrm{j}}} \mathrm{d} \omega
\end{aligned}
$$

Na mesma forma é possível obter um algoritmo que processe os coeficientes da função de escala da wavelet harmônicas, para isto é necessária a consideração da equação (4.52), aplicando a transformada inversa de Fourier nesta, usa-se a relação (4.20):

$$
\begin{aligned}
& \phi_{\mathrm{k}}=\int_{0}^{2 \pi} \Phi_{\mathrm{k}}(\omega) \mathrm{e}^{\mathrm{i} \omega \mathrm{t}} \mathrm{d} \omega \\
& \bar{\phi}_{\mathrm{k}}=\int_{0}^{2 \pi} \Phi_{\mathrm{k}}(-\omega) \mathrm{e}^{-\mathrm{i} \omega \mathrm{t}} \mathrm{d} \omega
\end{aligned}
$$

Aplicando a relação (4.86) em (4.73), assim como (4.85) em (4.74), e realizando os mesmos procedimentos para chegar em (4.83) e (4.84), tem-se:

$$
\begin{aligned}
& \mathrm{u}_{\mathrm{k}}=\int_{0}^{2 \pi} \mathrm{X}(\omega) \mathrm{e}^{i \omega \mathrm{k}} \mathrm{d} \omega \\
& \tilde{\mathrm{u}}_{\mathrm{k}}=\int_{0}^{2 \pi} \mathrm{X}(-\omega) \mathrm{e}^{-i \omega \mathrm{k}} \mathrm{d} \omega
\end{aligned}
$$

Agora para a obtenção dos coeficientes $\mathrm{w}_{\mathrm{j}, \mathrm{k}}, \mathrm{u}_{\mathrm{k}}, \tilde{\mathrm{w}}_{\mathrm{j}, \mathrm{k}}$ e $\tilde{\mathrm{u}}_{\mathrm{k}}$ numericamente, é necessário à substituição da integração pelo somatório, como a transformada de Fourier do sinal $\mathrm{x}(\mathrm{t})$ está na forma contínua, é necessário estabelecer as relações:

$$
\begin{aligned}
& \omega=2 \pi\left(2^{\mathrm{j}}+\mathrm{r}\right) \quad \text { para } \quad \mathrm{r}=0,1, \ldots,\left(2^{\mathrm{j}}-1\right) \\
& \mathrm{X}_{2^{\mathrm{j}}+\mathrm{r}}=2 \pi \mathrm{X}(\omega) \\
& \mathrm{X}_{0}=4 \pi \mathrm{X}(0)
\end{aligned}
$$

Utilizando a relação (4.90) em (4.83) e (4.84), assim como a relação (4.91) em (4.87) e (4.88), pois esta ultima representa a média, chega-se:

$$
\begin{aligned}
& \mathrm{w}_{\mathrm{j}, \mathrm{k}}=\frac{1}{2 \pi} \sum_{\mathrm{r}=0}^{2^{\mathrm{j}}-1} \mathrm{X}_{2^{\mathrm{j}}+\mathrm{r}} \mathrm{e}^{\mathrm{i} 2 \pi\left(2^{\mathrm{j}}+\mathrm{r}\right) \mathrm{k} / 2^{\mathrm{j}}} \Delta \omega \\
& \tilde{\mathrm{w}}_{\mathrm{j}, \mathrm{k}}=\frac{1}{2 \pi} \sum_{\mathrm{r}=0}^{2^{\mathrm{j}}-1} \mathrm{X}_{-\left(2^{\mathrm{j}+r}\right)} \mathrm{e}^{-\mathrm{i} 2 \pi\left(2^{\mathrm{j}}+\mathrm{r}\right) \mathrm{k} / 2^{\mathrm{j}}} \Delta \omega
\end{aligned}
$$


$\mathrm{u}_{0}=\frac{1}{2 \pi}\left(\mathrm{X}_{0} / 2\right) \Delta \omega$

$\tilde{\mathrm{u}}_{0}=\frac{1}{2 \pi}\left(\mathrm{X}_{0} / 2\right) \Delta \omega$

Como:

$\Delta \omega=2 \pi$

$\mathrm{e}^{\mathrm{i} 2 \pi \mathrm{k}}=1$

$\tilde{X}_{2^{\mathrm{j}}+\mathrm{r}}=\mathrm{X}_{-\left(2^{\mathrm{j}}+\mathrm{r}\right)}$

Resulta em:

$$
\begin{aligned}
& \mathrm{w}_{2^{\mathrm{j}}+\mathrm{k}}=\sum_{\mathrm{r}=0}^{2^{\mathrm{j}}-1} \mathrm{X}_{2^{\mathrm{j}+\mathrm{r}}} \mathrm{e}^{i 2 \pi \mathrm{rk} / 2^{\mathrm{j}}} \quad \text { para } \mathrm{k}=0,1, \ldots,\left(2^{\mathrm{j}}-1\right) \\
& \tilde{\mathrm{w}}_{2^{\mathrm{j}}+\mathrm{k}}=\sum_{\mathrm{r}=0}^{2^{\mathrm{j}}-1} \tilde{\mathrm{X}}_{2^{\mathrm{j}+\mathrm{r}}} \mathrm{e}^{-i 2 \pi \mathrm{rk} / 2^{\mathrm{j}}} \quad \text { para } \mathrm{k}=0,1, \ldots,\left(2^{\mathrm{j}}-1\right) \\
& \mathrm{u}_{0}=\mathrm{X}_{0} / 2 \\
& \tilde{\mathrm{u}}_{0}=\mathrm{X}_{0} / 2
\end{aligned}
$$

As equações (4.101) e (4.102) somadas geram a amplitude média do sinal, quando se analisa as equações (4.99) e (4.100) percebe-se que são equivalentes a IDFT, a qual é representada pela equação (3.67), com a diferença de $\mathrm{N}$, onde este é substituído por $2^{\mathrm{j}}$, isto significa que a DHWT de um sinal x(t) é obtida através de várias IDFT deste sinal, ocorrendo em intervalos de $2^{\mathrm{j}}$ (ou níveis, como são usualmente chamados) para cada coeficiente. Por ultimo é necessário considerar:

$\mathrm{w}_{\mathrm{N} / 2}=\mathrm{X}_{\mathrm{N} / 2}$

Onde (4.103) não é considerada nas equações (4.99) e (4.100), mas esta é necessária para assegurar o teorema de Parseval, como será visto adiante. Quando as equações (4.99) a (4.103) são utilizadas, representam a DHWT. Para a reconstrução ou geração de um sinal $\mathrm{x}(\mathrm{t})$ através da DHWT, primeiramente é necessária a DFT de $\mathrm{x}(\mathrm{t})$, esta pode ser obtida através da DFT de DHWT a cada intervalo $2^{\mathrm{j}}$, o que representa o caminho inverso de (4.99) a (4.103): 


$$
\begin{aligned}
& \mathrm{X}_{2^{\mathrm{j}}+\mathrm{r}}=\frac{1}{2^{\mathrm{j}}} \sum_{\mathrm{k}=0}^{2^{\mathrm{j}}-1} \mathrm{w}_{2^{\mathrm{j}+\mathrm{k}}} \mathrm{e}^{-i 2 \pi \mathrm{rk} / 2^{\mathrm{j}}} \quad \text { para } \mathrm{r}=0,1, \ldots,\left(2^{\mathrm{j}}-1\right) \\
& \tilde{\mathrm{X}}_{2^{\mathrm{j}}+\mathrm{r}}=\frac{1}{2^{\mathrm{j}}} \sum_{\mathrm{k}=0}^{2^{\mathrm{j}}-1} \tilde{\mathrm{w}}_{2^{\mathrm{j}+\mathrm{k}}} \mathrm{e}^{i 2 \pi \mathrm{rk} / 2^{\mathrm{j}}} \quad \text { para } \mathrm{r}=0,1, \ldots,\left(2^{\mathrm{j}}-1\right) \\
& \mathrm{X}_{0}=2 \mathrm{u}_{0} \\
& \mathrm{X}_{0}=2 \tilde{\mathrm{u}}_{0} \\
& \mathrm{X}_{\mathrm{N} / 2}=\mathrm{w}_{\mathrm{N} / 2}
\end{aligned}
$$

A partir de (4.106) e (4.107), pode-se chegar às seguintes equações:

$$
\begin{aligned}
& \mathrm{u}_{0}=\tilde{\mathrm{u}}_{0} \\
& \mathrm{X}_{0}=\mathrm{u}_{0}+\tilde{\mathrm{u}}_{0}=\mathrm{u}
\end{aligned}
$$

Em poder de todo o espectro da DFT, é possível a obtenção do sinal $\mathrm{x}(\mathrm{t})$, através da IDFT usual, como representada em (3.67), todo o processo descrito é equivalente a IDHWT.

Como é necessária a utilização de pontos em quantidade equivalente a uma potência de 2 , a aplicação da FFT é bastante útil, pois alem da maior eficiência, não são necessários truncamentos ou adição de valores nulos no sinal. Segundo Newland (1993), o algoritmo piramidal de Mallat, é um algoritmo seqüencial no sentido que os níveis sucessivos da wavelet de Daubechies são computados em seqüência.

Ao contrário, o algoritmo da FFT para a wavelet harmônica é um algoritmo em paralelo, este é implementado primeiramente para toda seqüência, em seguida, para cada nível, onde pode ser computado simultaneamente. Para aplicações que envolvam grandes sequiências, pode ter uma vantagem significativa em relação à wavelet de Daubechies. 


\subsection{WAVELET HARMÔNICA GENERALIZADA}

Na wavelet harmônica clássica desenvolvida por Newland (1993), possui uma variação do domínio do seu espectro de freqüência que aumenta em um fator 2 , esta wavelet descreve muito bem sinais com parcelas de alta freqüência com curta duração e baixa freqüência com longa duração, no entanto, embora este tipo de sinal tenha maior ocorrência, outros tipos de sinais não serão bem detalhados com o uso desta wavelet, sendo então necessária uma flexibilização da variação do domínio do espectro de freqüência.

Devido a isto, Newland (1994) desenvolveu uma versão generalizada da wavelet harmônica que pode ser aplicada para um melhoramento da resolução de alguns sinais, através da flexibilização de suas funções base. Então, assim como a wavelet harmônica clássica, pode-se obter esta versão generalizada através da definição do espectro de frequiência da wavelet harmônica generalizada:

$$
\Psi_{n, m}(\omega)=\mid \begin{array}{lll}
\frac{1}{2 \pi(n-m)} & \text { se } & 2 \pi m \leq \omega<2 \pi n \\
0 & \text { se } & \omega<2 \pi m \text { ou } \omega \geq 2 \pi n
\end{array}
$$

Onde:

$|n|>|m|>1 \quad$ para $\quad n, m \in \mathrm{R}-[-1,1]$

Com a aplicação da Transformada inversa de Fourier em (4.111), tem-se:

$\psi(\mathrm{t})=\left(\mathrm{e}^{i 2 \pi n \mathrm{t}}-\mathrm{e}^{i 2 \pi m \mathrm{t}}\right) / i 2 \pi(n-m) \mathrm{t}$

Para a geração das wavelets harmônicas generalizadas filhas, é utilizada a definição do seguinte espectro de freqüência:

$$
\Psi_{n, m, \mathrm{k}}(\omega)=\mid \begin{array}{lll}
\frac{1}{2 \pi(n-m)} \mathrm{e}^{\frac{-i \omega \mathrm{k}}{(n-m)}} & \text { se } & 2 \pi m \leq \omega<2 \pi n \\
0 & \text { se } & \omega<2 \pi m \text { ou } \omega \geq 2 \pi n
\end{array}
$$

Com a aplicação da Transformada inversa de Fourier em (4.114), tem-se:

$$
\psi((n-m) \mathrm{t}-\mathrm{k})=\left(\mathrm{e}^{i 2 \pi n((n-m) \mathrm{t}-\mathrm{k}) /(n-m)}-\mathrm{e}^{i 2 \pi m((n-m) \mathrm{t}-\mathrm{k}) /(n-m)}\right) / i 2 \pi((n-m) \mathrm{t}-\mathrm{k})
$$


Embora estas wavelets harmônicas generalizadas filhas sejam ortogonais, para haver a geração da DHWT, os valores de m e n necessitam ser inteiros, e por uma questão de simplificação, este trabalho desenvolveu a seguinte notação:

$$
\Psi_{\mathrm{j}, \mathrm{k}}(\omega)=\mid \begin{array}{lll}
\frac{1}{2 \pi\left(\alpha_{\mathrm{j}+1}-\alpha_{\mathrm{j}}\right)} \mathrm{e}^{\frac{-i \omega \mathrm{k}}{\left(\alpha_{\mathrm{j}+1}-\alpha_{\mathrm{j}}\right)}} & \text { se } & 2 \pi \alpha_{\mathrm{j}} \leq \omega<2 \pi \alpha_{\mathrm{j}+1} \\
0 & \text { se } & \omega<2 \pi \alpha_{\mathrm{j}} \text { ou } \omega \geq 2 \pi \alpha_{\mathrm{j}+1}
\end{array}
$$

Sendo $\alpha$ a representação de um vetor ou uma função, que tem a função de indicar a posição do domínio não nulo do espectro de freqüência da wavelet harmônica generalizada filha, tal que:

$$
\begin{array}{lll}
\alpha_{\mathrm{j}}=1 & \text { para } & \mathrm{j}=0 \\
\alpha_{\mathrm{j}+1}-\alpha_{\mathrm{j}} \geq 1 & \text { para } & \mathrm{j}, \alpha_{\mathrm{j}} \text { e } \alpha_{\mathrm{j}+1} \in \mathrm{N}
\end{array}
$$

Onde (4.117) e (4.118) são necessárias para garantir a ortogonalidade do espectro de freqüência das wavelets filhas, e consequentemente garantir a ortogonalidade entre as wavelets filhas, como relatado anteriormente. Por uma questão de simplificação, (4.118) pode ser representada na forma:

$$
\Delta \alpha_{\mathrm{j}}=\alpha_{\mathrm{j}+1}-\alpha_{\mathrm{j}}
$$

Com a aplicação da Transformada inversa de Fourier em (4.116), e utilizando a relação (4.119), tem-se:

$$
\psi\left(\Delta \alpha_{\mathrm{j}} \mathrm{t}-\mathrm{k}\right)=\left(\mathrm{e}^{i 2 \pi \alpha_{\mathrm{j}+1}\left(\Delta \alpha_{\mathrm{j}} \mathrm{t}-\mathrm{k}\right) / \Delta \alpha_{\mathrm{j}}}-\mathrm{e}^{i 2 \pi \alpha_{\mathrm{j}}\left(\Delta \alpha_{\mathrm{j}} \mathrm{t}-\mathrm{k}\right) / \Delta \alpha_{\mathrm{j}}}\right) / i 2 \pi\left(\Delta \alpha_{\mathrm{j}} \mathrm{t}-\mathrm{k}\right)
$$

Onde (4.120) é a wavelet harmônica filha generalizada, igualmente a wavelet harmônica clássica, possui seu conjugado complexo:

$$
\bar{\psi}\left(\Delta \alpha_{\mathrm{j}} \mathrm{t}-\mathrm{k}\right)=\left(\mathrm{e}^{-i 2 \pi \alpha_{\mathrm{j}}\left(\Delta \alpha_{\mathrm{j}} \mathrm{t}-\mathrm{k}\right) / \Delta \alpha_{\mathrm{j}}}-\mathrm{e}^{-i 2 \pi \alpha_{\mathrm{j}+1}\left(\Delta \alpha_{\mathrm{j}} \mathrm{t}-\mathrm{k}\right) / \Delta \alpha_{\mathrm{j}}}\right) / i 2 \pi\left(\Delta \alpha_{\mathrm{j}} \mathrm{t}-\mathrm{k}\right)
$$

E realizando uma modificação em (4.12), chega-se:

$$
\psi_{\mathrm{j}, \mathrm{k}}(\mathrm{t})=\Delta \alpha_{\mathrm{j}}^{1 / 2} \psi\left(\Delta \alpha_{\mathrm{j}} \mathrm{x}-\mathrm{k}\right)
$$


O que resulta em:

$$
\begin{aligned}
& \psi_{\mathrm{j}, \mathrm{k}}(\mathrm{t})=\Delta \alpha_{\mathrm{j}}^{1 / 2}\left(\mathrm{e}^{i 2 \pi \alpha_{\mathrm{j}+1}\left(\Delta \alpha_{\mathrm{j}} \mathrm{t}-\mathrm{k}\right) / \Delta \alpha_{\mathrm{j}}}-\mathrm{e}^{i 2 \pi \alpha_{\mathrm{j}}\left(\Delta \alpha_{\mathrm{j}} \mathrm{t}-\mathrm{k}\right) / \Delta \alpha_{\mathrm{j}}}\right) / i 2 \pi\left(\Delta \alpha_{\mathrm{j}} \mathrm{t}-\mathrm{k}\right) \\
& \bar{\psi}_{\mathrm{j}, \mathrm{k}}(\mathrm{t})=\Delta \alpha_{\mathrm{j}}^{1 / 2}\left(\mathrm{e}^{-i 2 \pi \alpha_{\mathrm{j}}\left(\Delta \alpha_{\mathrm{j}} \mathrm{t}-\mathrm{k}\right) / \Delta \alpha_{\mathrm{j}}}-\mathrm{e}^{-i 2 \pi \alpha_{\mathrm{j}+1}\left(\Delta \alpha_{\mathrm{j}} \mathrm{t}-\mathrm{k}\right) / \Delta \alpha_{\mathrm{j}}}\right) / i 2 \pi\left(\Delta \alpha_{\mathrm{j}} \mathrm{t}-\mathrm{k}\right)
\end{aligned}
$$

Assim como as wavelets harmônicas clássicas, as wavelets harmônicas generalizadas constituem uma família de funções ortogonais, onde esta também surge de uma relação entre o produto interno de duas wavelets harmônicas generalizadas quaisquer, com o produto interno de suas respectivas transformadas, representadas pelos espectros de freqüência (Figura 4.11).

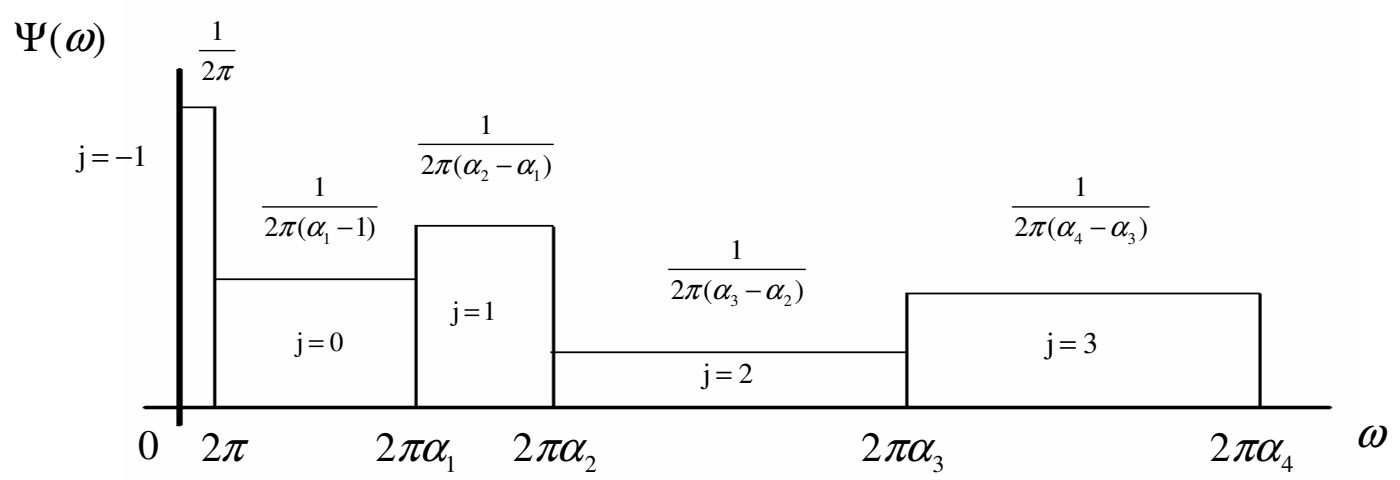

Figura 4.11. Espectro de freqüência obtido através de Transformadas de Fourier de wavelets harmônicas de diferentes níveis.

Como o produto interno de suas transformadas é nulo, este valor se repete para o produto interno das wavelets harmônicas garantindo a sua ortogonalidade, por fim, a relação (4.14) garante a normalidade da wavelet harmônica filha. Então, devido a ortonormalidade, as relações (4.13) e (4.21) são validas, assim como as relações (4.61) a (4.70).

Para a reconstrução ou geração de um sinal, tendo as funções base, é necessário saber os valores das ponderações destas funções, para isto, usa-se a Transformada discreta da wavelet harmônica ou DHWT (Discrete Harmonic Wavelet Transform), assim como estas wavelets, existem duas DHWT para cada par de j e k, devido a isto, são definidos os coeficientes para as wavelets harmônicas generalizadas:

$$
\begin{aligned}
& \mathrm{w}_{\mathrm{j}, \mathrm{k}}=\Delta \alpha_{\mathrm{j}}^{1 / 2} \int_{-\infty}^{+\infty} \mathrm{x}(\mathrm{t}) \bar{\Psi}_{\mathrm{j}, \mathrm{k}} \mathrm{dt} \\
& \tilde{\mathrm{w}}_{\mathrm{j}, \mathrm{k}}=\Delta \alpha_{\mathrm{j}}^{1 / 2} \int_{-\infty}^{+\infty} \mathrm{x}(\mathrm{t}) \psi_{\mathrm{j}, \mathrm{k}} \mathrm{dt}
\end{aligned}
$$


Os sinais que podem ser representados por esta wavelet também obedecem a condição (4.1), com isto, a transformada inversa discreta da wavelet harmônica ou IDHWT (Inverse Discret Harmonic Wavelet Transform) para o caso generalizado pode ser expressa por:

$$
\mathrm{x}(\mathrm{t})=\left[\begin{array}{l}
\sum_{\mathrm{k}=-\infty}^{+\infty}\left(\mathrm{u}_{\mathrm{k}} \phi(\mathrm{t}-\mathrm{k})+\tilde{\mathrm{u}}_{\mathrm{k}} \bar{\phi}(\mathrm{t}-\mathrm{k})\right)+ \\
\sum_{\mathrm{j}=0}^{+\infty} \sum_{\mathrm{k}=-\infty}^{+\infty}\left(\mathrm{w}_{\mathrm{j}, \mathrm{k}} \psi\left(\Delta \alpha_{\mathrm{j}} \mathrm{t}-\mathrm{k}\right)+\tilde{\mathrm{w}}_{\mathrm{j}, \mathrm{k}} \bar{\psi}\left(\Delta \alpha_{\mathrm{j}} \mathrm{t}-\mathrm{k}\right)\right)
\end{array}\right]
$$

Inúmeras variações de espectros de freqüência gerados através da transformada de Fourier que satisfaçam as relações (4.117) e (4.118) podem ser utilizados, no entanto, para este trabalho é sugerido dois tipos possíveis de espectros de freqüência, estes são baseados em variações de domínios dadas por progressões geométricas e progressões aritméticas.

Para as progressões geométricas, com o intuito de um melhor entendimento, é utilizada a seguinte mudança de notação:

$$
\mathrm{Pg}_{\mathrm{j}}=\alpha_{\mathrm{j}}
$$

Então, baseado em (4.119), tem-se:

$$
\Delta \mathrm{Pg}_{\mathrm{j}}=\mathrm{Pg}_{\mathrm{j}+1}-\mathrm{Pg}_{\mathrm{j}}
$$

Onde:

$$
\begin{array}{lll}
\Delta \mathrm{Pg}_{\mathrm{j}}=\mathrm{q}^{\mathrm{j}} & \text { para } & \mathrm{q} \in \mathrm{N}-\{1\} \\
\operatorname{Pg}_{\mathrm{j}+1}=\mathrm{Pg}_{\mathrm{j}}+\mathrm{q}^{\mathrm{j}} & \text { para } & \mathrm{j} \in \mathrm{Z}_{+} \\
\operatorname{Pg}_{\mathrm{j}}=1 & \text { para } & \mathrm{j}=0
\end{array}
$$

A partir disto, é possível definir a transformada de Fourier da wavelet harmônica filha:

$$
\Psi_{\mathrm{j}, \mathrm{k}}(\omega)=\mid \begin{array}{lll}
\frac{1}{2 \pi\left(\Delta \mathrm{Pg}_{\mathrm{j}}\right)} \mathrm{e}^{\frac{-i \omega \mathrm{k}}{\Delta \mathrm{Pg}_{\mathrm{j}}}} & \text { se } & 2 \pi\left(\operatorname{Pg}_{\mathrm{j}}\right) \leq \omega<2 \pi\left(\mathrm{Pg}_{\mathrm{j}+1}\right) \\
0 & \text { se } & \omega<2 \pi\left(\operatorname{Pg}_{\mathrm{j}}\right) \text { ou } \omega \geq 2 \pi\left(\operatorname{Pg}_{\mathrm{j}+1}\right)
\end{array}
$$


Com a aplicação da Transformada inversa de Fourier em (4.116), e utilizando a relação (4.128), tem-se:

$\psi\left(\left(\Delta \mathrm{Pg}_{\mathrm{j}}\right) \mathrm{t}-\mathrm{k}\right)=\left(\mathrm{e}^{i 2 \pi\left(\mathrm{Pg}_{\mathrm{j}+1}\right)\left(\left(\Delta \mathrm{Pg}_{\mathrm{j}}\right) \mathrm{t}-\mathrm{k}\right) / \Delta \mathrm{Pg}_{\mathrm{j}}}-\mathrm{e}^{i 2 \pi\left(\mathrm{Pg}_{\mathrm{j}}\right)\left(\left(\Delta \mathrm{Pg}_{\mathrm{j}}\right) \mathrm{t}-\mathrm{k}\right) / \Delta \mathrm{Pg}_{\mathrm{j}}}\right) / i 2 \pi\left(\left(\Delta \mathrm{Pg}_{\mathrm{j}}\right) \mathrm{t}-\mathrm{k}\right)$

Novamente com o uso da relação (4.128) aplicada em (4.125) e (4.126), chega-se a DHWT com espectro de freqüência variando em escala geométrica:

$$
\begin{aligned}
& \mathrm{w}_{\mathrm{j}, \mathrm{k}}=\left(\Delta \mathrm{Pg}_{\mathrm{j}}\right)^{1 / 2} \int_{-\infty}^{+\infty} \mathrm{x}(\mathrm{t}) \bar{\Psi}_{\mathrm{j}, \mathrm{k}} \mathrm{dt} \\
& \tilde{\mathrm{w}}_{\mathrm{j}, \mathrm{k}}=\left(\Delta \mathrm{Pg}_{\mathrm{j}}\right)^{1 / 2} \int_{-\infty}^{+\infty} \mathrm{x}(\mathrm{t}) \Psi_{\mathrm{j}, \mathrm{k}} \mathrm{dt}
\end{aligned}
$$

A IDHWT para o caso de espectro de freqüência com variação geométrica pode ser expressa por:

$$
\mathrm{x}(\mathrm{t})=\left[\begin{array}{l}
\sum_{\mathrm{k}=-\infty}^{+\infty}\left(\mathrm{u}_{\mathrm{k}} \phi(\mathrm{t}-\mathrm{k})+\tilde{\mathrm{u}}_{\mathrm{k}} \bar{\phi}(\mathrm{t}-\mathrm{k})\right)+ \\
\sum_{\mathrm{j}=0}^{+\infty} \sum_{\mathrm{k}=-\infty}^{+\infty}\left(\mathrm{w}_{\mathrm{j}, \mathrm{k}} \psi\left(\left(\Delta \mathrm{Pg}_{\mathrm{j}}\right) \mathrm{t}-\mathrm{k}\right)+\tilde{\mathrm{w}}_{\mathrm{j}, \mathrm{k}} \bar{\psi}\left(\left(\Delta \mathrm{Pg}_{\mathrm{j}}\right) \mathrm{t}-\mathrm{k}\right)\right)
\end{array}\right]
$$

Percebe-se que a variação do espectro de freqüência da wavelet harmônica clássica é um caso particular deste, pois basta utilizar $q=2$ para obter o espectro de freqüência clássico. Para as progressões aritméticas, também com o intuito de um melhor entendimento, é utilizada a seguinte mudança de notação:

$$
\mathrm{Pa}_{\mathrm{j}}=\alpha_{\mathrm{j}}
$$

Então, baseado em (4.119), tem-se:

$$
\Delta \mathrm{Pa}_{\mathrm{j}}=\mathrm{Pa}_{\mathrm{j}+1}-\mathrm{Pa}_{\mathrm{j}}
$$

Onde:

$$
\begin{array}{lll}
\Delta \mathrm{Pa}_{\mathrm{j}}=\mathrm{p}(\mathrm{j}+1) & \text { para } & \mathrm{p} \in \mathrm{N} \\
\mathrm{Pa}_{\mathrm{j}+1}=\mathrm{Pa}_{\mathrm{j}}+\mathrm{p}(\mathrm{j}+1) & \text { para } & \mathrm{j} \in \mathrm{Z}_{+} \\
\mathrm{Pa}_{\mathrm{j}}=1 & \text { para } & \mathrm{j}=0
\end{array}
$$


A partir disto, é possível definir a transformada de Fourier da wavelet harmônica filha:

$$
\Psi_{j, \mathrm{k}}(\omega)=\mid \begin{array}{lll}
\frac{1}{2 \pi\left(\Delta \mathrm{Pa}_{\mathrm{j}}\right)} \mathrm{e}^{\frac{-i \omega \mathrm{k}}{\Delta \mathrm{Pa}_{\mathrm{j}}}} & \text { se } & 2 \pi\left(\mathrm{Pa}_{\mathrm{j}}\right) \leq \omega<2 \pi\left(\mathrm{Pa}_{\mathrm{j}+1}\right) \\
0 & \text { se } & \omega<2 \pi\left(\mathrm{Pa}_{\mathrm{j}}\right) \text { ou } \omega \geq 2 \pi\left(\mathrm{Pa}_{\mathrm{j}+1}\right)
\end{array}
$$

Com a aplicação da Transformada inversa de Fourier em (4.116), e utilizando a relação (4.138), tem-se:

$\psi\left(\left(\Delta \mathrm{Pa}_{\mathrm{j}}\right) \mathrm{t}-\mathrm{k}\right)=\left(\mathrm{e}^{i 2 \pi\left(\mathrm{Pa}_{\mathrm{j}+1}\right)\left(\left(\Delta \mathrm{Pa}_{\mathrm{j}}\right) \mathrm{t}-\mathrm{k}\right) / \Delta \mathrm{Pa}_{\mathrm{j}}}-\mathrm{e}^{i 2 \pi\left(\mathrm{Pa}_{\mathrm{j}}\right)\left(\left(\Delta \mathrm{Pa}_{\mathrm{j}}\right) \mathrm{t}-\mathrm{k}\right) / \Delta \mathrm{Pa}_{\mathrm{j}}}\right) / i 2 \pi\left(\left(\Delta \mathrm{Pa}_{\mathrm{j}}\right) \mathrm{t}-\mathrm{k}\right)$

Novamente com o uso da relação (4.138) aplicada em (4.125) e (4.126), chega-se a DHWT com espectro de freqüência variando em escala aritmética:

$$
\begin{aligned}
& \mathrm{w}_{\mathrm{j}, \mathrm{k}}=\left(\Delta \mathrm{Pa}_{\mathrm{j}}\right)^{1 / 2} \int_{-\infty}^{+\infty} \mathrm{x}(\mathrm{t}) \bar{\Psi}_{\mathrm{j}, \mathrm{k}} \mathrm{dt} \\
& \tilde{\mathrm{w}}_{\mathrm{j}, \mathrm{k}}=\left(\Delta \mathrm{Pa}_{\mathrm{j}}\right)^{1 / 2} \int_{-\infty}^{+\infty} \mathrm{x}(\mathrm{t}) \Psi_{\mathrm{j}, \mathrm{k}} \mathrm{dt}
\end{aligned}
$$

A IDHWT para o caso de espectro de freqüência com variação aritmética pode ser expressa por:

$$
\mathrm{x}(\mathrm{t})=\left[\begin{array}{l}
\sum_{\mathrm{k}=-\infty}^{+\infty}\left(\mathrm{u}_{\mathrm{k}} \phi(\mathrm{t}-\mathrm{k})+\tilde{\mathrm{u}}_{\mathrm{k}} \bar{\phi}(\mathrm{t}-\mathrm{k})\right)+ \\
\sum_{\mathrm{j}=0}^{+\infty} \sum_{\mathrm{k}=-\infty}^{+\infty}\left(\mathrm{w}_{\mathrm{j}, \mathrm{k}} \psi\left(\left(\Delta \mathrm{Pa}_{\mathrm{j}}\right) \mathrm{t}-\mathrm{k}\right)+\tilde{\mathrm{w}}_{\mathrm{j}, \mathrm{k}} \bar{\psi}\left(\left(\Delta \mathrm{Pa} \mathrm{a}_{\mathrm{j}}\right) \mathrm{t}-\mathrm{k}\right)\right)
\end{array}\right]
$$




\subsection{ANÁLISE NUMÉRICA DA WAVELET HARMÔNICA GENERALIZADA}

As wavelets harmônicas generalizadas possuem muitas das características das wavelets harmônicas clássicas, como a definição precisa no domínio da freqüência em sua transformada de Fourier, no entanto, este domínio pode ser mais bem controlado, permitindo assim, uma análise mais detalhada dos sinais que não se ajustem a uma taxa de variação de freqüência de fator 2 .

Para gerar um algoritmo mais eficiente que processe os coeficientes da wavelet harmônica generalizada, primeiro, é necessária a utilização de valores racionais no domínio da freqüência da transformada de Fourier desta wavelet, com isto, e com a consideração da equação (4.116), aplica-se a transformada inversa de Fourier nesta, usando a relação (4.122):

$$
\begin{aligned}
& \psi_{\mathrm{j}, \mathrm{k}}=\Delta \alpha_{\mathrm{j}}^{1 / 2} \int_{2 \pi \alpha_{\mathrm{j}}}^{2 \pi \alpha_{\mathrm{j}+1}} \Psi_{\mathrm{j}, \mathrm{k}}(\omega) \mathrm{e}^{\mathrm{i} \omega t} \mathrm{~d} \omega \\
& \bar{\psi}_{\mathrm{j}, \mathrm{k}}=\Delta \alpha_{\mathrm{j}}^{1 / 2} \int_{2 \pi \alpha_{\mathrm{j}}}^{2 \pi \alpha_{\mathrm{j}+1}} \Psi_{\mathrm{j}, \mathrm{k}}(-\omega) \mathrm{e}^{-\mathrm{i} \omega \mathrm{t}} \mathrm{d} \omega
\end{aligned}
$$

Aplicando a relação (4.148) em (4.126), assim como (4.149) em (4.125), tem-se:

$$
\begin{aligned}
& \mathrm{w}_{\mathrm{j}, \mathrm{k}}=\Delta \alpha_{\mathrm{j}} \int_{-\infty}^{+\infty} \mathrm{x}(\mathrm{t}) \int_{2 \pi \alpha_{\mathrm{j}}}^{2 \pi \alpha_{\mathrm{j}+1}} \Psi_{\mathrm{j}, \mathrm{k}}(-\omega) \mathrm{e}^{-\mathrm{i} \omega \mathrm{t}} \mathrm{d} \omega \mathrm{dt} \\
& \tilde{\mathrm{w}}_{\mathrm{j}, \mathrm{k}}=\Delta \alpha_{\mathrm{j}} \int_{-\infty}^{+\infty} \mathrm{x}(\mathrm{t}) \int_{2 \pi \alpha_{\mathrm{j}}}^{2 \pi \alpha_{\mathrm{j}+1}} \Psi_{\mathrm{j}, \mathrm{k}}(\omega) \mathrm{e}^{\mathrm{i} \omega \mathrm{t}} \mathrm{d} \omega \mathrm{dt}
\end{aligned}
$$

Abrindo (4.116) e realizando alguma manipulação algébrica, chega-se:

$$
\begin{aligned}
& \mathrm{w}_{\mathrm{j}, \mathrm{k}}=\int_{2 \pi \alpha_{\mathrm{j}}}^{2 \pi \alpha_{\mathrm{j}+1}}\left(\frac{1}{2 \pi} \int_{-\infty}^{+\infty} \mathrm{x}(\mathrm{t}) \mathrm{e}^{-i \omega \mathrm{t}} \mathrm{dt}\right) \mathrm{e}^{i \omega \mathrm{k} / \Delta \alpha_{\mathrm{j}}} \mathrm{d} \omega \\
& \tilde{\mathrm{w}}_{\mathrm{j}, \mathrm{k}}=\int_{2 \pi \alpha_{\mathrm{j}}}^{2 \pi \alpha_{\mathrm{j}+1}}\left(\frac{1}{2 \pi} \int_{-\infty}^{+\infty} \mathrm{x}(\mathrm{t}) \mathrm{e}^{i \omega \mathrm{t}} \mathrm{dt}\right) \mathrm{e}^{-i \omega \mathrm{k} / \Delta \alpha_{\mathrm{j}}} \mathrm{d} \omega
\end{aligned}
$$

Percebe-se que em (4.152) e (4.153), as partes entre parênteses são as Transformada de Fourier como apresentado em (3.34), com isto:

$$
\mathrm{w}_{\mathrm{j}, \mathrm{k}}=\int_{2 \pi \alpha_{\mathrm{j}}}^{2 \pi \alpha_{\mathrm{j}+1}} \mathrm{X}(\omega) \mathrm{e}^{i \omega \mathrm{k} / \Delta \alpha_{\mathrm{j}}} \mathrm{d} \omega
$$


$\tilde{\mathrm{W}}_{\mathrm{j}, \mathrm{k}}=\int_{2 \pi \alpha_{\mathrm{j}}}^{2 \pi \alpha_{\mathrm{j}+1}} \mathrm{X}(-\omega) \mathrm{e}^{-i \omega \mathrm{k} / \Delta \alpha_{\mathrm{j}}} \mathrm{d} \omega$

Agora para a obtenção dos coeficientes $\mathrm{w}_{\mathrm{j}, \mathrm{k}}, \mathrm{u}_{\mathrm{k}}, \tilde{\mathrm{w}}_{\mathrm{j}, \mathrm{k}}$ e $\tilde{\mathrm{u}}_{\mathrm{k}}$ numericamente, é necessário à substituição da integração pelo somatório, como a transformada de Fourier do sinal $\mathrm{x}(\mathrm{t})$ está na forma contínua, é necessário estabelecer as relações:

$$
\begin{aligned}
& \omega=2 \pi\left(\alpha_{\mathrm{j}}+\mathrm{r}\right) \quad \text { para } \quad \mathrm{r}=0,1, \ldots,\left(\Delta \alpha_{\mathrm{j}}-1\right) \\
& \mathrm{X}_{\alpha_{\mathrm{j}}+\mathrm{r}}=2 \pi \mathrm{X}(\omega)
\end{aligned}
$$

Utilizando a relação (4.157) em (4.154) e (4.155), chega-se:

$$
\begin{aligned}
& \mathrm{w}_{\mathrm{j}, \mathrm{k}}=\frac{1}{2 \pi} \sum_{\mathrm{r}=0}^{\Delta \alpha_{\mathrm{j}}-1} \mathrm{X}_{\alpha_{\mathrm{j}}+\mathrm{r}} \mathrm{e}^{\mathrm{i} 2 \pi\left(\alpha_{\mathrm{j}}+\mathrm{r}\right) \mathrm{k} / \Delta \alpha_{\mathrm{j}}} \Delta \omega \\
& \tilde{\mathrm{w}}_{\mathrm{j}, \mathrm{k}}=\frac{1}{2 \pi} \sum_{\mathrm{r}=0}^{\Delta \alpha_{\mathrm{j}}-1} \mathrm{X}_{-\left(\alpha_{\mathrm{j}}+\mathrm{r}\right)} \mathrm{e}^{-\mathrm{i} 2 \pi\left(\alpha_{\mathrm{j}}+\mathrm{r}\right) \mathrm{k} / \Delta \alpha_{\mathrm{j}}} \Delta \omega
\end{aligned}
$$

Como:

$$
\begin{aligned}
& \Delta \omega=2 \pi \\
& \tilde{\mathrm{X}}_{\alpha_{\mathrm{j}}+\mathrm{r}}=\mathrm{X}_{-\left(\alpha_{\mathrm{j}}+\mathrm{r}\right)}
\end{aligned}
$$

Resulta em:

$$
\begin{aligned}
& \mathrm{w}_{\alpha_{\mathrm{j}}+\mathrm{k}}=\sum_{\mathrm{r}=0}^{\Delta \alpha_{\mathrm{j}}-1} \mathrm{X}_{\alpha_{\mathrm{j}}+\mathrm{r}} \mathrm{e}^{\mathrm{i} 2 \pi\left(\alpha_{\mathrm{j}}+\mathrm{r}\right) \mathrm{k} / \Delta \alpha_{\mathrm{j}}} \quad \text { para } \quad \mathrm{k}=0,1, \ldots,\left(\Delta \alpha_{\mathrm{j}}-1\right) \\
& \tilde{\mathrm{w}}_{\alpha_{\mathrm{j}}+\mathrm{k}}=\sum_{\mathrm{r}=0}^{\Delta \alpha_{\mathrm{j}}-1} \tilde{\mathrm{X}}_{\alpha_{\mathrm{j}}+\mathrm{r}} \mathrm{e}^{-\mathrm{i} 2 \pi\left(\alpha_{\mathrm{j}}+\mathrm{r}\right) \mathrm{k} / \Delta \alpha_{\mathrm{j}}} \quad \text { para } \quad \mathrm{k}=0,1, \ldots,\left(\Delta \alpha_{\mathrm{j}}-1\right)
\end{aligned}
$$

Diferentemente da wavelet harmônica clássica, tem-se:

$$
\mathrm{e}^{\mathrm{i} 2 \pi \alpha_{\mathrm{j}} \mathrm{k} / \Delta \alpha_{\mathrm{j}}} \neq 1 \quad \text { para } \quad \alpha_{\mathrm{j}} / \Delta \alpha_{\mathrm{j}} \in \mathrm{Q}_{+}^{*}
$$

No entanto, pode-se isolar (4.164), de forma a possibilitar o uso da IDFT para a obtenção da DHWT:

$$
\mathrm{w}_{\alpha_{\mathrm{j}}+\mathrm{k}}=\mathrm{e}^{\mathrm{i} 2 \pi \alpha_{\mathrm{j}} \mathrm{k} / \Delta \alpha_{\mathrm{j}}}\left(\sum_{\mathrm{r}=0}^{\Delta \alpha_{\mathrm{j}}-1} \mathrm{X}_{\alpha_{\mathrm{j}}+\mathrm{r}} \mathrm{e}^{\mathrm{i} 2 \pi \mathrm{rk} / \Delta \alpha_{\mathrm{j}}}\right) \quad \text { para } \quad \mathrm{k}=0,1, \ldots,\left(\Delta \alpha_{\mathrm{j}}-1\right)
$$


$\tilde{\mathrm{w}}_{\alpha_{\mathrm{j}}+\mathrm{k}}=\mathrm{e}^{-\mathrm{i} 2 \pi \alpha_{\mathrm{j}} \mathrm{k} / \Delta \alpha_{\mathrm{j}}}\left(\sum_{\mathrm{r}=0}^{\Delta \alpha_{\mathrm{j}}-1} \tilde{\mathrm{X}}_{\alpha_{\mathrm{j}}+\mathrm{r}} \mathrm{e}^{-\mathrm{i} 2 \pi \mathrm{r} \mathrm{k} / \Delta \alpha_{\mathrm{j}}}\right) \quad$ para $\quad \mathrm{k}=0,1, \ldots,\left(\Delta \alpha_{\mathrm{j}}-1\right)$

E fazendo as seguintes relações:

$$
\begin{array}{lll}
w_{\alpha_{\mathrm{j}}+\mathrm{k}}=\mathrm{w}_{\alpha_{\mathrm{j}}+\mathrm{k}} \mathrm{e}^{-\mathrm{i} 2 \pi \alpha_{\mathrm{j}} \mathrm{k} / \Delta \alpha_{\mathrm{j}}} & \text { para } & \mathrm{k}=0,1, \ldots,\left(\Delta \alpha_{\mathrm{j}}-1\right) \\
\tilde{w}_{\alpha_{\mathrm{j}}+\mathrm{k}}=\tilde{\mathrm{w}}_{\alpha_{\mathrm{j}}+\mathrm{k}} \mathrm{e}^{\mathrm{i} 2 \pi \alpha_{\mathrm{j}} \mathrm{k} / \Delta \alpha_{\mathrm{j}}} & \text { para } & \mathrm{k}=0,1, \ldots,\left(\Delta \alpha_{\mathrm{j}}-1\right)
\end{array}
$$

Onde (4.167) e (4.168) são usadas para a reconstrução ou geração de um sinal x(t) através da DHWT, que pode ser obtida com a DFT de (4.169) e (4.170) a cada intervalo $\Delta \alpha_{\mathrm{j}}$, obtendo assim, a DFT do sinal $\mathrm{x}(\mathrm{t})$ :

$$
\begin{array}{lll}
\mathrm{X}_{\alpha_{\mathrm{j}}+\mathrm{r}}=\frac{1}{\Delta \alpha_{\mathrm{j}}} \sum_{\mathrm{k}=0}^{\Delta \alpha_{\mathrm{j}}-1} w_{\alpha_{\mathrm{j}} \mathrm{k}} \mathrm{e}^{-i 2 \pi \mathrm{r} / \Delta \alpha_{\mathrm{j}}} & \text { para } & \mathrm{r}=0,1, \ldots,\left(\Delta \alpha_{\mathrm{j}}-1\right) \\
\tilde{\mathrm{X}}_{\alpha_{\mathrm{j}}+\mathrm{r}}=\frac{1}{\Delta \alpha_{\mathrm{j}}} \sum_{\mathrm{k}=0}^{\Delta \alpha_{\mathrm{j}}-1} \tilde{w}_{\alpha_{\mathrm{j}}+\mathrm{k}} \mathrm{e}^{i 2 \pi \mathrm{rk} / \Delta \alpha_{\mathrm{j}}} & \text { para } & \mathrm{r}=0,1, \ldots,\left(\Delta \alpha_{\mathrm{j}}-1\right)
\end{array}
$$

Como a função de escala para a wavelet harmônica possui espectro único para a freqüência, as relações (4.106), (4.107), (4.109) e (4.110) são válidas. Assim como a relação para o coeficiente na posição N/2, mostrado em (4.108) também é válida para wavelet harmônica generalizada, pois o teorema de Parseval também precisa ser atendido. Para os casos particulares de wavelets harmônicas mencionadas neste trabalho, a aplicação para a DHWT, com espectro de freqüência variando em progressão geométrica, resulta:

$$
\begin{aligned}
& \mathrm{W}_{\mathrm{Pg}_{\mathrm{j}}+\mathrm{k}}=\mathrm{e}^{\mathrm{Pg}_{\mathrm{j}} \mathrm{k} / \Delta \mathrm{Pg}_{\mathrm{j}}}\left(\sum_{\mathrm{r}=0}^{\mathrm{Pg}_{\mathrm{j}}-1} \mathrm{X}_{\mathrm{Pg}_{\mathrm{j}}+\mathrm{r}} \mathrm{e}^{\mathrm{i} 2 \pi \mathrm{rk} / \Delta \mathrm{Pg}_{\mathrm{j}}}\right) \quad \text { para } \quad \mathrm{k}=0,1, \ldots,\left(\Delta \mathrm{Pg}_{\mathrm{j}}-1\right) \\
& \tilde{\mathrm{W}}_{\mathrm{Pg}_{\mathrm{j}}+\mathrm{k}}=\mathrm{e}^{-\mathrm{Pg}_{\mathrm{j}} \mathrm{k} / \Delta \mathrm{Pg}_{\mathrm{j}}}\left(\sum_{\mathrm{r}=0}^{\Delta \mathrm{Pg}_{\mathrm{j}}-1} \tilde{\mathrm{X}}_{\mathrm{Pg}_{\mathrm{j}}+\mathrm{r}} \mathrm{e}^{-\mathrm{i} 2 \pi \mathrm{r} / \Delta \mathrm{Pg}_{\mathrm{j}}}\right) \text { para } \mathrm{k}=0,1, \ldots,\left(\Delta \mathrm{Pg}_{\mathrm{j}}-1\right)
\end{aligned}
$$

E a reconstrução do sinal a partir da DFT:

$$
\begin{array}{lll}
w_{\mathrm{Pg}_{\mathrm{j}}+\mathrm{k}}=\mathrm{w}_{\mathrm{Pg}_{\mathrm{j}}+\mathrm{k}} \mathrm{e}^{-\mathrm{i} 2 \pi \mathrm{Pg}_{\mathrm{j}} \mathrm{k} / \Delta \mathrm{Pg}_{\mathrm{j}}} & \text { para } & \mathrm{k}=0,1, \ldots,\left(\Delta \mathrm{Pg}_{\mathrm{j}}-1\right) \\
\tilde{w}_{\mathrm{Pg}_{\mathrm{j}}+\mathrm{k}}=\tilde{\mathrm{w}}_{\mathrm{Pg}_{\mathrm{j}}+\mathrm{k}} \mathrm{e}^{\mathrm{i} 2 \pi \mathrm{Pg}_{\mathrm{j}} \mathrm{k} / \Delta \mathrm{Pg}_{\mathrm{j}}} & \text { para } & \mathrm{k}=0,1, \ldots,\left(\Delta \mathrm{Pg}_{\mathrm{j}}-1\right)
\end{array}
$$




$$
\begin{array}{lll}
\mathrm{X}_{\mathrm{Pg}_{\mathrm{j}}+\mathrm{r}}=\frac{1}{\Delta \mathrm{Pg} g_{\mathrm{j}}} \sum_{\mathrm{k}=0}^{\Delta \alpha_{\mathrm{j}}-1} w_{\mathrm{Pg}_{\mathrm{j}}+\mathrm{k}} \mathrm{e}^{-i 2 \pi \mathrm{rk} / \Delta \mathrm{Pg}_{\mathrm{j}}} & \text { para } & \mathrm{r}=0,1, \ldots,\left(\Delta \mathrm{Pg}_{\mathrm{j}}-1\right) \\
\tilde{\mathrm{X}}_{\mathrm{Pg}_{\mathrm{j}}+\mathrm{r}}=\frac{1}{\Delta \mathrm{Pg}} \sum_{\mathrm{k}=0}^{\Delta \mathrm{Pg}_{\mathrm{j}}-1} \tilde{w}_{\mathrm{Pg}_{\mathrm{j}}+\mathrm{k}} \mathrm{e}^{i 2 \pi \mathrm{rk} / \Delta \mathrm{Pg}_{\mathrm{j}}} & \text { para } & \mathrm{r}=0,1, \ldots,\left(\Delta \mathrm{Pg}_{\mathrm{j}}-1\right)
\end{array}
$$

Para a DHWT, com espectro de freqüência variando em progressão aritmética, resulta:

$$
\begin{aligned}
& \mathrm{W}_{\mathrm{Pa}_{\mathrm{j}}+\mathrm{k}}=\mathrm{e}^{\mathrm{Pa}_{\mathrm{j}} \mathrm{k} / \Delta \mathrm{Pa}_{\mathrm{j}}}\left(\sum_{\mathrm{r}=0}^{\mathrm{Pg}_{\mathrm{j}}-1} \mathrm{X}_{\mathrm{Pa}_{\mathrm{j}}+\mathrm{r}} \mathrm{e}^{\mathrm{i} 2 \pi \mathrm{rk} / \Delta \mathrm{Pa}_{\mathrm{j}}}\right) \quad \text { para } \quad \mathrm{k}=0,1, \ldots,\left(\Delta \mathrm{Pa}_{\mathrm{j}}-1\right) \\
& \tilde{\mathrm{W}}_{\mathrm{Pa}_{\mathrm{j}}+\mathrm{k}}=\mathrm{e}^{-\mathrm{Pa}_{\mathrm{j}} \mathrm{k} / \Delta \mathrm{Pa}_{\mathrm{j}}}\left(\sum_{\mathrm{r}=0}^{\Delta \mathrm{Pa}_{\mathrm{j}}-1} \tilde{\mathrm{X}}_{\mathrm{Pa}_{\mathrm{j}}+\mathrm{r}} \mathrm{e}^{-\mathrm{i} 2 \pi \mathrm{rk} / \Delta \mathrm{Pa}_{\mathrm{j}}}\right) \text { para } \mathrm{k}=0,1, \ldots,\left(\Delta \mathrm{Pa}_{\mathrm{j}}-1\right)
\end{aligned}
$$

E a reconstrução do sinal a partir da DFT:

$$
\begin{array}{lll}
w_{\mathrm{Pa}_{\mathrm{j}}+\mathrm{k}}=\mathrm{w}_{\mathrm{Pa}_{\mathrm{j}}+\mathrm{k}} \mathrm{e}^{-\mathrm{i} 2 \pi \mathrm{Pa}_{\mathrm{j}} \mathrm{k} / \Delta \mathrm{Pa}_{\mathrm{j}}} & \text { para } & \mathrm{k}=0,1, \ldots,\left(\Delta \mathrm{Pa}_{\mathrm{j}}-1\right) \\
\tilde{w}_{\mathrm{Pa}_{\mathrm{j}}+\mathrm{k}}=\tilde{\mathrm{w}}_{\mathrm{Pa}_{\mathrm{j}}+\mathrm{k}} \mathrm{e}^{\mathrm{i} 2 \pi \mathrm{Pa}_{\mathrm{j}} \mathrm{k} / \Delta \mathrm{Pa}_{\mathrm{j}}} & \text { para } & \mathrm{k}=0,1, \ldots,\left(\Delta \mathrm{Pa}_{\mathrm{j}}-1\right) \\
\mathrm{X}_{\mathrm{Pa}_{\mathrm{j}}+\mathrm{r}}=\frac{1}{\Delta \mathrm{Pa}_{\mathrm{j}}} \sum_{\mathrm{k}=0}^{\Delta \mathrm{Pa}_{\mathrm{j}}-1} w_{\mathrm{Pa}_{\mathrm{j}} \mathrm{k}} \mathrm{e}^{-i 2 \pi \mathrm{rk} / \Delta \mathrm{Pa}_{\mathrm{j}}} & \text { para } & \mathrm{r}=0,1, \ldots,\left(\Delta \mathrm{Pa}_{\mathrm{j}}-1\right) \\
\tilde{\mathrm{X}}_{\mathrm{Pa}_{\mathrm{j}}+\mathrm{r}}=\frac{1}{\Delta \mathrm{Pa} \mathrm{a}_{\mathrm{j}}} \sum_{\mathrm{k}=0}^{\Delta \mathrm{Pg}_{\mathrm{j}}-1} \tilde{w}_{\mathrm{Pa}_{\mathrm{j}}+\mathrm{k}} \mathrm{e}^{i 2 \pi \mathrm{rk} / \Delta \mathrm{Pa}_{\mathrm{j}}} & \text { para } & \mathrm{r}=0,1, \ldots,\left(\Delta \mathrm{Pa}_{\mathrm{j}}-1\right)
\end{array}
$$

A desvantagem do uso de wavelet harmônica, com domínios do espectro de Fourier variando de forma diferente da clássica, decorre do fato que a quantidade de valores gerados para os coeficientes da DHWT serem distintos de um fator de 2, o que resulta na relação (4.164), implicando em uma quantidade maior de operações e dificultando o uso da FFT, pois passa a ser necessária a inclusão de zeros para preencher valores até uma quantidade de fator 2 .

No entanto, as vantagens residem na flexibilidade das janelas, permitindo uma regulagem a critério das necessidades do pesquisador, com um algoritmo ainda em paralelo. 


\subsection{ESCALOGRAMA}

Assim como a Transformada de Fourier possui espectros que relacionam as amplitudes e as potências dos sinais com suas respectivas freqüências, as Transformadas janeladas de Fourier e as Transformadas de wavelet possuem espectros que relacionam estes dados não apenas no domínio da frequiência, mas também do tempo, sendo chamados de espectogramas.

Quando se utiliza wavelets ortogonais, o espectograma pode ser chamado de escalograma, ou de acordo com Newland (1993), Mapa de média quadrada (meansquare map). Os espectogramas podem ser utilizados para descreverem transformadas contínuas ou discretas, mas independente do tipo da transformada, o volume do espectograma é equivalente ao valor do quadrado médio do sinal analisado.

Comparando a Transformada janelada de Fourier com a Transformada de wavelet através dos espectogramas discretos, é observado que a primeira possui janelas tempofreqüência sempre com as mesmas dimensões, sendo estas dimensões predefinidas.

As wavelets, pelo contrário, possuem janelas que captam um intervalo grande de tempo para um intervalo pequeno de freqüências mais baixas, onde não é necessário um bom detalhamento no tempo e captam um intervalo pequeno de tempo para um intervalo grande de frequiências mais altas, onde se faz necessário um melhor detalhamento no tempo (Figura 4.12(a) e 4.12(b)).

(a)

STFT

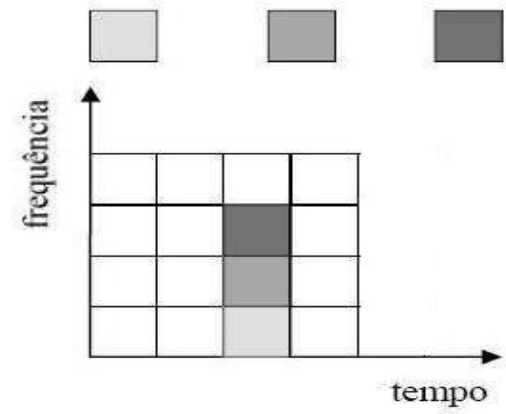

(b)

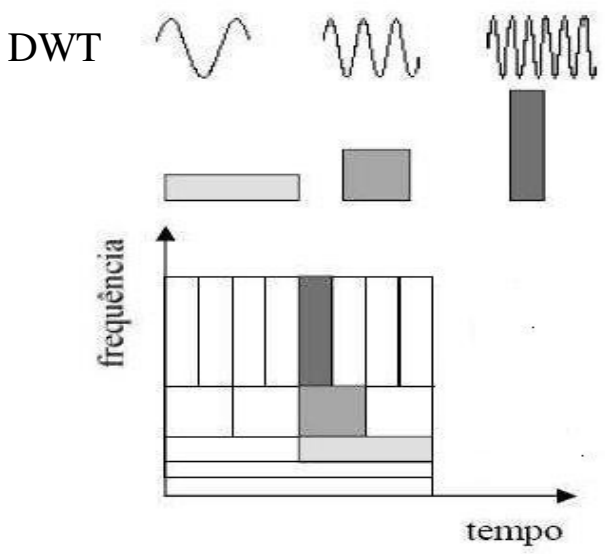

Figura 4.12. Janelas tempo-frequência: (a) Fourier janelada; (b) Wavelet (Imagem modificada a partir de Barbosa; Blitzkow (2008) ). 
Para a obtenção do escalograma a partir das wavelets harmônicas, parte-se da definição que o volume do escalograma é equivalente a média quadrática, então a partir disto, com a aplicação do teorema de Parseval em (3.41) na sua forma discreta, tem-se:

$\frac{1}{\mathrm{~N}} \sum_{\mathrm{r}=0}^{\mathrm{N}-1}\left|\mathrm{X}_{\mathrm{r}}\right|^{2}=\sum_{\mathrm{k}=0}^{\mathrm{N}-1}\left|\mathrm{X}_{\mathrm{k}}\right|^{2}$

Sendo $\mathrm{x}_{\mathrm{r}}$ que aparece a esquerda de (4.183) equivalente a (4.75), representada desta vez na forma mais utilizada para as wavelets harmônicas:

$\mathrm{x}_{\mathrm{r}}=\left[\begin{array}{l}\left(\mathrm{u}_{0} \phi(\Delta \mathrm{r}-\mathrm{k})+\tilde{\mathrm{u}}_{0} \bar{\phi}(\Delta \mathrm{r}-\mathrm{k})\right)+ \\ \sum_{\mathrm{j}=0}^{\mathrm{n}-2} \sum_{\mathrm{k}=0}^{2^{\mathrm{j}}-1}\left(\mathrm{w}_{2^{\mathrm{j}}+\mathrm{k}} \psi\left(2^{\mathrm{j}} \Delta \mathrm{r}-\mathrm{k}\right)+\tilde{\mathrm{w}}_{2^{\mathrm{j}}+\mathrm{k}} \bar{\psi}\left(2^{\mathrm{j}} \Delta \mathrm{r}-\mathrm{k}\right)\right)\end{array}\right]$

Onde $\mathrm{N}=2^{\mathrm{n}}$, como estas wavelets são ortogonais, a integrais dos produtos das wavelets ou funções de escalas serão nulas de acordo com as relações (4.13), (4.21) e (4.61) a (4.66), isto resulta em:

$\frac{1}{\mathrm{~N}} \sum_{\mathrm{r}=0}^{\mathrm{N}-1}\left|\mathrm{x}_{\mathrm{r}}\right|^{2}=|\mathrm{u}|^{2}+\sum_{\mathrm{j}=0}^{\mathrm{n}-2} \frac{1}{2^{\mathrm{j}}} \sum_{\mathrm{k}=0}^{2^{\mathrm{j}}-1}\left(\left|\mathrm{w}_{2^{\mathrm{j}}+\mathrm{k}}\right|^{2}+\left|\tilde{\mathrm{w}}_{2^{\mathrm{j}}+\mathrm{k}}\right|^{2}\right)+\left|\mathrm{w}_{\mathrm{N} / 2}\right|^{2}$

Quando o sinal é representado apenas por números reais, (4.185) pode ser representada por:

$\frac{1}{\mathrm{~N}} \sum_{\mathrm{r}=0}^{\mathrm{N}-1}\left|\mathrm{x}_{\mathrm{r}}\right|^{2}=|\mathrm{u}|^{2}+\sum_{\mathrm{j}=0}^{\mathrm{n}-2} \frac{1}{2^{\mathrm{j}}} \sum_{\mathrm{k}=0}^{2^{\mathrm{j}}-1}\left(\left|\mathrm{w}_{2^{\mathrm{j}}+\mathrm{k}}\right|^{2}+\left|\overline{\mathrm{w}}_{2^{\mathrm{j}}+\mathrm{k}}\right|^{2}\right)+\left|\mathrm{w}_{\mathrm{N} / 2}\right|^{2}$

Para o caso da wavelet generalizada, $\mathrm{x}_{\mathrm{r}}$ pode ser representado na forma:

$\mathrm{x}_{\mathrm{r}}=\left[\begin{array}{l}\left(\mathrm{u}_{0} \phi(\Delta \mathrm{r}-\mathrm{k})+\tilde{\mathrm{u}}_{0} \bar{\phi}(\Delta \mathrm{r}-\mathrm{k})\right)+ \\ \sum_{\mathrm{j}=0}^{\mathrm{n}-2} \sum_{\mathrm{k}=0}^{\Delta \alpha_{\mathrm{j}}-1}\left(\mathrm{w}_{\alpha_{\mathrm{j}}+\mathrm{k}} \psi\left(\alpha_{\mathrm{j}} \Delta \mathrm{r}-\mathrm{k}\right)+\tilde{\mathrm{w}}_{\alpha_{\mathrm{j}}+\mathrm{k}} \bar{\psi}\left(\alpha_{\mathrm{j}} \Delta \mathrm{r}-\mathrm{k}\right)\right)\end{array}\right]$

Equivalente a (4.186), o teorema de Parseval pode ser representado como:

$\frac{1}{\mathrm{~N}} \sum_{\mathrm{r}=0}^{\mathrm{N}-1}\left|\mathrm{x}_{\mathrm{r}}\right|^{2}=|\mathrm{u}|^{2}+\sum_{\mathrm{j}=0}^{\mathrm{n}-2} \frac{1}{\Delta \alpha_{\mathrm{j}}} \sum_{\mathrm{k}=0}^{\Delta \alpha_{\mathrm{j}}-1}\left(\left|\mathrm{w}_{\alpha_{\mathrm{j}}+\mathrm{k}}\right|^{2}+\left|\tilde{\mathrm{w}}_{\alpha_{\mathrm{j}}+\mathrm{k}}\right|^{2}\right)+\left|\mathrm{w}_{\mathrm{N} / 2}\right|^{2}$ 
Para os casos particulares de janelas tempo-frequiência com variação geométrica e aritmética, baseados em (4.188), podem ser representados, respectivamente:

$$
\begin{aligned}
& \frac{1}{\mathrm{~N}_{\mathrm{Pg}}} \sum_{\mathrm{r}=0}^{\mathrm{N}_{\mathrm{Pg}}-1}\left|\mathrm{x}_{\mathrm{r}}\right|^{2}=|\mathrm{u}|^{2}+\sum_{\mathrm{j}=0}^{\mathrm{n}-2} \frac{1}{\Delta \mathrm{Pg}_{\mathrm{j}}} \sum_{\mathrm{k}=0}^{\Delta \mathrm{Pg}_{\mathrm{j}}-1}\left(\left|\mathrm{w}_{\mathrm{Pg}_{\mathrm{j}}+\mathrm{k}}\right|^{2}+\left|\tilde{\mathrm{W}}_{\mathrm{Pg}_{\mathrm{j}}+\mathrm{k}}\right|^{2}\right)+\left|\mathrm{W}_{\mathrm{N}_{\mathrm{Pg}} / 2}\right|^{2} \\
& \frac{1}{\mathrm{~N}_{\mathrm{Pa}}} \sum_{\mathrm{r}=0}^{\mathrm{N}_{\mathrm{Pa}}-1}\left|\mathrm{x}_{\mathrm{r}}\right|^{2}=|\mathrm{u}|^{2}+\sum_{\mathrm{j}=0}^{\mathrm{n}-2} \frac{1}{\Delta \mathrm{Pa}_{\mathrm{j}}} \sum_{\mathrm{k}=0}^{\Delta \mathrm{Pa}_{\mathrm{j}}-1}\left(\left|\mathrm{w}_{\mathrm{Pa}_{\mathrm{j}}+\mathrm{k}}\right|^{2}+\left|\tilde{\mathrm{w}}_{\mathrm{Pa}_{\mathrm{j}}+\mathrm{k}}\right|^{2}\right)+\mid \mathrm{W}_{\left.\mathrm{N}_{\mathrm{Pa}_{\mathrm{a}} / 2}\right|^{2}}
\end{aligned}
$$

Onde as variáveis $\mathrm{N}_{\mathrm{Pg}}$ e $\mathrm{N}_{\mathrm{Pa}}$ representam, respectivamente, a quantidade de pontos do sinal ou coeficientes da DHWT. As equações (4.185), (4.186), (4.188) a (4.190) podem ser ilustradas em gráficos tridimensionais, onde os valores das amplitudes das wavelets harmônicas e das funções de escala são fornecidas ao quadrado, e o domínio do tempo possui um intervalo unitário, sendo este domínio relacionado com os valores de $\mathrm{k}$, e o domínio da freqüência passa a ser representado por ciclos em intervalos unitários, sendo este relacionado com os valores de j. Para um melhor entendimento, são mostrados dois exemplos, com janelas de variação geométrica e aritmética respectivamente, ambas para $\mathrm{n}=4$ (Figura 4.13.(a) e 4.13.(b)).

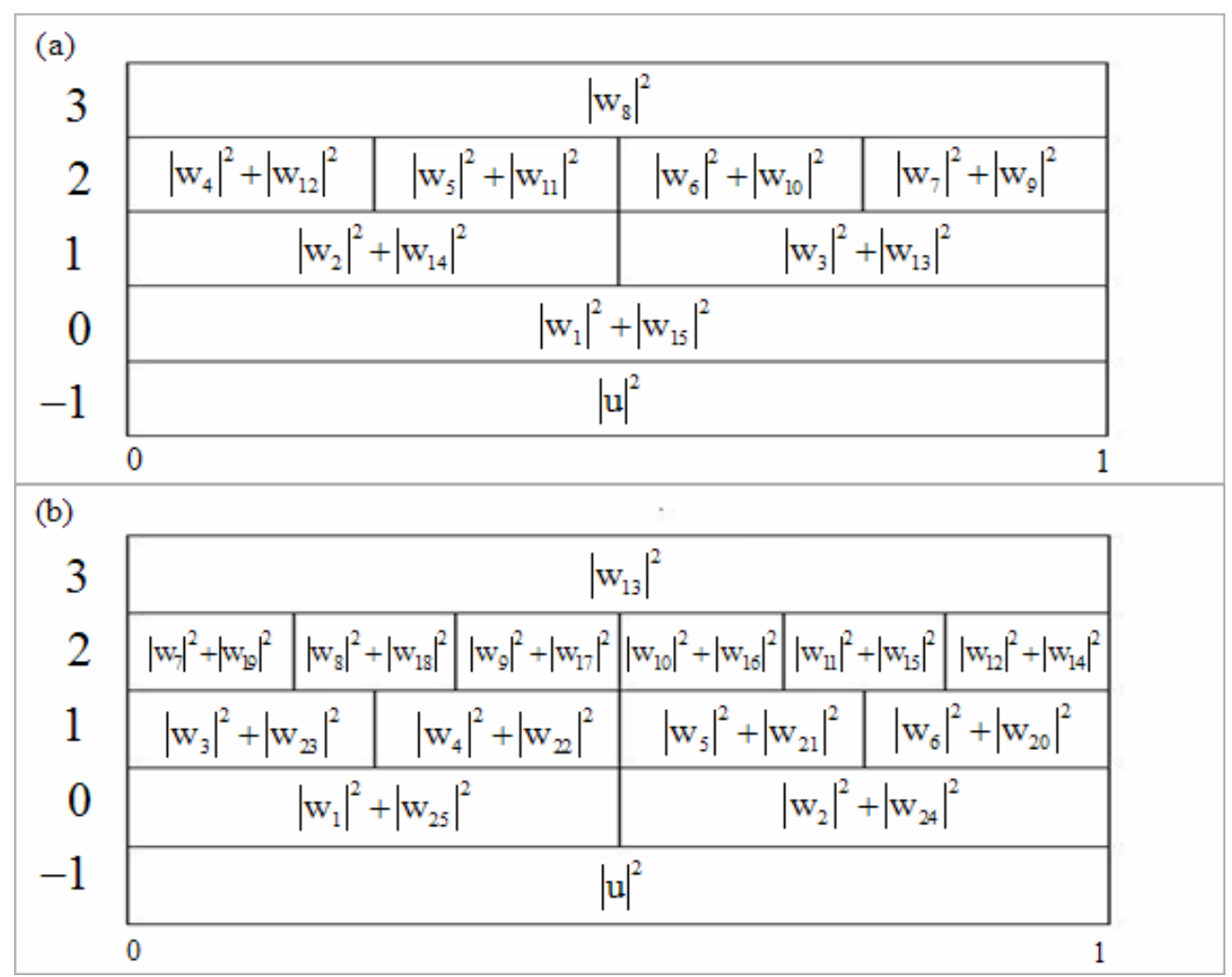

Figura 4.13. Arrumação no escalograma dos coeficientes da wavelet harmônica: (a) com janela tempo-freqüência de variação geométrica para q=2; (b) com janela tempofrequiência de variação aritmética para $\mathrm{p}=2$. 
Como pode ser visto em 4.11.(a), para $\mathrm{q}=2$, onde coincide com a janela tempofreqüência clássica, em cada nível de j, com exceções do primeiro e do último, as frequiências dobram de valores, devido a isto, as wavelets são espaçadas em $1 / 2^{\mathrm{j}}$ ou $1 / \Delta \mathrm{Pg}_{\mathrm{j}}$ ao longo do tempo, possuindo assim $2^{\mathrm{j}}$ ou $\Delta \mathrm{Pg}_{\mathrm{j}}$ wavelets filhas por nível.

Em relação a 4.11.(b), para p=2, também com exceções do primeiro e do último, a quantidade de janelas aumentam em 2 para cada nível de $\mathrm{j}$, assim como o intervalo de freqüência, devido a isto, as wavelets são espaçadas em $1 / \Delta \mathrm{Pa}_{\mathrm{j}}$ ao longo do tempo, possuindo então $\Delta \mathrm{Pa}_{\mathrm{j}}$ wavelets filhas por nível.

Os volumes destes escalogramas são equivalentes aos valores da média quadrática do sinal se estes forem feitos de prismas de bases retangulares representando cada janela, dado pelo produto de cada intervalo unitário no domínio da freqüência, pelo intervalo $1 / \Delta \mathrm{Pg}_{\mathrm{j}}$ ou $1 / \Delta \mathrm{Pa}_{\mathrm{j}}$ no domínio do tempo, com uma altura definida pelo valor de amplitude ao quadrado.

Isto significa que o volume do escalograma representa a energia do sinal, se forem analisadas seções das amplitudes em relação às freqüências para determinados instantes de tempo, a área de cada seção representa a potência do sinal para um determinado instante de tempo, esta é uma informação extremamente importante quando se trata de um comportamento não-estacionário.

Quando a área e o formato da seção no escalograma são idênticos para qualquer instante de tempo, dentro de um intervalo de tempo convertido para unitário, o sinal é dito, segundo Morettin (1999), localmente estacionário. Um comportamento estacionário não poderia ser representado por um escalograma, pois seria necessário que o intervalo de tempo fosse infinito, assim o volume, resultando em energia infinita para o escalograma, o que caracterizaria junto com as seções constantes ao longo do tempo, um comportamento estacionário. Quando se deseja obter a DHWT com variações geométricas e aritméticas de janelas tempo-frequiência, assim como a DHWT clássica, é necessário o uso de certa quantidade de pontos do sinal analisado. 
Para a obtenção dos valores de $\mathrm{N}_{\mathrm{Pg}}$ e $\mathrm{N}_{\mathrm{Pa}}$, a partir da quantidade de níveis, é possível recorrer às equações básicas da soma dos termos das progressões geométricas e aritméticas com algumas considerações, pois cada janela, com exceção da primeira e da ultima possui um par de coeficientes, e devido ao fato do valor inicial de $\mathrm{j}$ ser igual à zero, resulta em valor máximo do nível de (n-1). Com estas considerações, têm-se as equações, respectivamente, para a progressão geométrica e aritmética:

$$
\begin{array}{lll}
\mathrm{N}_{\mathrm{Pg}}=2 \frac{\left(\mathrm{q}^{(\mathrm{n}-1)}-1\right)}{\mathrm{q}-1}+2 & \text { para } & \mathrm{n} \in \mathrm{N} \\
\mathrm{N}_{\mathrm{Pa}}=\mathrm{p}(\mathrm{n}-1) \mathrm{n}+2 & \text { para } & \mathrm{n} \in \mathrm{N}
\end{array}
$$

Porém, quando se deseja saber a quantidade de níveis necessários a partir da quantidade de pontos analisados de um sinal, com o uso de alguma manipulação algébrica de (4.191) e (4.192), chega-se, respectivamente, para a progressão geométrica e aritmética:

$$
\begin{array}{lll}
\mathrm{n}=\log _{\mathrm{q}}\left[\frac{\left(\mathrm{N}_{\mathrm{Pg}}-2\right)(\mathrm{q}-1)}{2}+1\right]+1 & \text { para } & \mathrm{N}_{\mathrm{Pg}} \in \mathrm{N} \\
\mathrm{n}=\frac{\mathrm{p}+\sqrt{\mathrm{p}\left(\mathrm{p}+4 \mathrm{~N}_{\mathrm{Pa}}-8\right)}}{2 \mathrm{p}} & \text { para } & \mathrm{N}_{\mathrm{Pa}} \in \mathrm{N}
\end{array}
$$

Sendo os valores de $\mathrm{N}_{\mathrm{Pg}}$ e $\mathrm{N}_{\mathrm{Pa}}$ válidos apenas quando resultam em valores inteiros para n. Abaixo pode ser vista a relação entre a quantidade de coeficientes da DHWT e a quantidade de níveis da mesma (Tabela 4.1):

Tabela 4.1. Quantidade de coeficientes da DHWT em relação à quantidade de níveis para diferentes janelas tempo-freqüência.

\begin{tabular}{|r|r|r|r|r|}
\hline \multirow{2}{*}{$\mathrm{n}$} & \multicolumn{2}{|c|}{$\mathrm{N}_{\mathrm{Pg}}$} & \multicolumn{2}{|c|}{$\mathrm{N}_{\mathrm{Pa}}$} \\
\cline { 2 - 6 } & $\mathrm{q}=2$ & $\mathrm{q}=3$ & $\mathrm{p}=2$ & $\mathrm{p}=10$ \\
\hline 1 & 2 & 2 & 2 & 2 \\
\hline 2 & 4 & 4 & 6 & 62 \\
\hline 3 & 8 & 10 & 14 & 122 \\
\hline 4 & 16 & 28 & 26 & 202 \\
\hline 5 & 32 & 82 & 42 & 302 \\
\hline 6 & 64 & 244 & 62 & 422 \\
\hline 7 & 128 & 730 & 86 & \\
\hline
\end{tabular}


A escolha de qual tipo funções base utilizadas cabe a necessidade do sinal analisado, pois, nota-se que para as parcelas dos sinais de baixas frequiências com durações não tão longas, a DHWT da janela com variação aritmética é mais adequada, devido ao fato de possuir mais coeficientes para valores baixos de n em relação à de variação geométrica.

No entanto, a taxa de crescimento de uma variação geométrica é consideravelmente maior, o que resulta uma escolha mais adequada para as parcelas dos sinais de altas frequiências com durações bem curtas, pois estes podem possuir mais coeficientes em sua DHWT do que a com variação aritmética para valores altos de n.

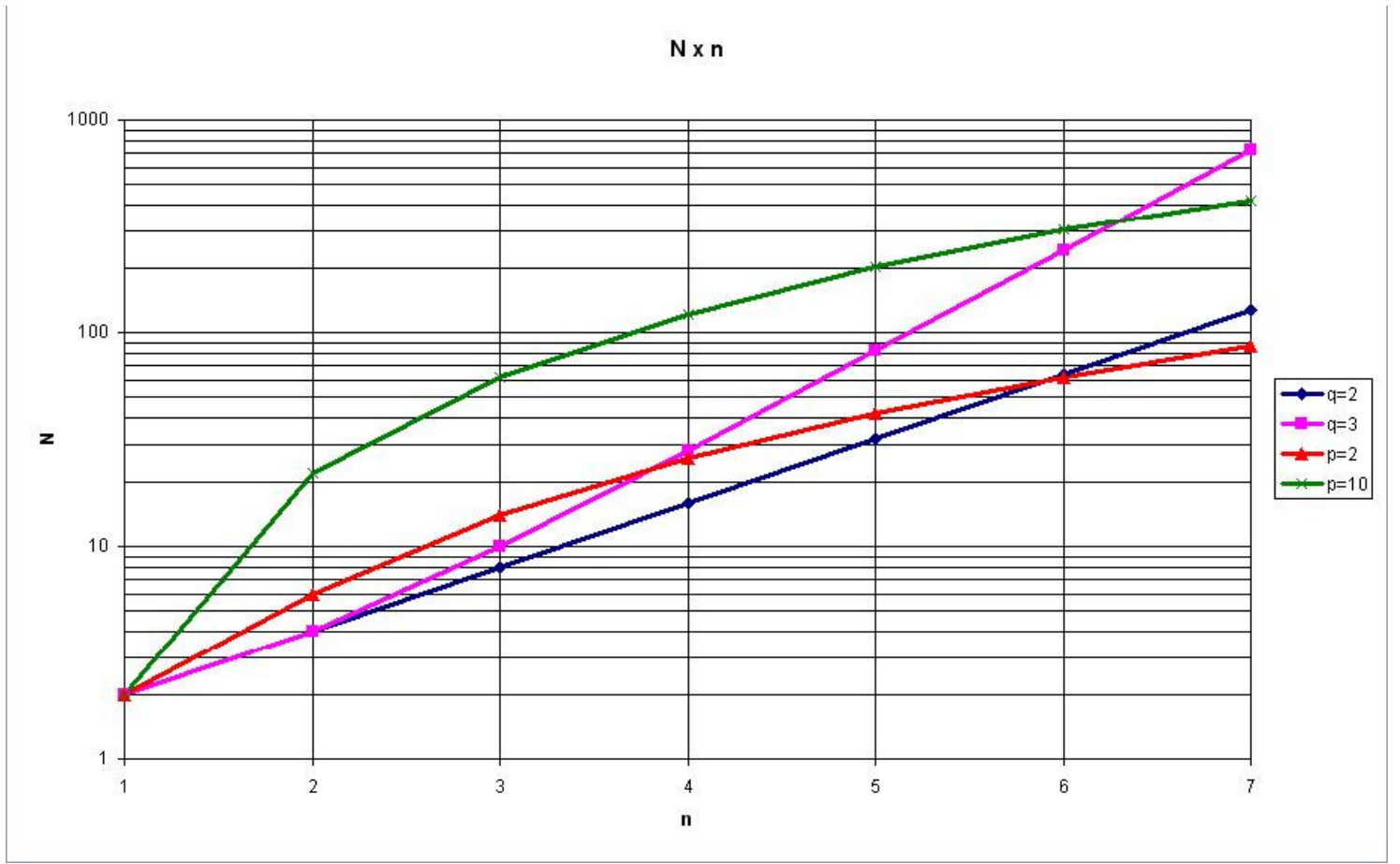

Figura 4.14. Gráfico gerado com os dados da tabela 4.1 o qual relaciona a quantidade de coeficientes $\mathrm{N}$ (escala logarítmica) da DHWT em relação à quantidade de níveis $\mathrm{n}$.

Estas relações ficam mais evidentes pela Figura 4.14, pois para as curvas das DHWTs com $\mathrm{p}=2$ e $\mathrm{p}=10$, há uma maior concentração de coeficientes, e consequentemente, um maior detalhamento para os baixos valores de n, no entanto, com o aumento do valor destes, as curvas das DHWTs com $\mathrm{q}=2$ e $\mathrm{q}=3$ cruzam respectivamente as curvas das DHWTs com $\mathrm{p}=2$ e $\mathrm{p}=10$, mostrando uma maior concentração de coeficientes, e consequentemente, um maior detalhamento para os altos valores de $\mathrm{n}$. 
Finalmente, pode-se chegar à conclusão que, para um mesmo sinal, com a mesma quantidade de pontos analisados, é necessária uma escolha de precisão entre o tempo e a freqüência, e é exatamente isto que os diversos tipos de variações de janelas do escalograma fazem, pois, fazendo uma comparação, se for usada à janela clássica como referência, a janela com variação aritmética atenua suas características e a janela com variação geométrica acentua suas características, cabendo ao pesquisador a escolha da janela que melhor atenda seus objetivos de visualização de dados. 


\section{ANÁLISE DE AÇÕES ALEATÓRIAS}

\subsection{INTRODUÇÃO}

Este capítulo procura desenvolver uma metodologia para geração de ações aleatórias não estacionárias, pois os modelos atuais, procuram manter os valores sempre livres para alcançar seus históricos máximos ao longo do tempo, isto ocorre devido a ao comportamento próximo do estacionário que estes possuem, sendo um comportamento o qual não é verídico em alguns tipos de solicitação aleatórias encontradas na natureza.

Estes carregamentos superdimensionados, em uma análise numérica da estrutura, podem resultar em um dimensionamento mais conservador, e consequentemente em maiores custos de materiais. Um carregamento artificial, melhor dimensionado com as características do carregamento real, poderia balancear melhor a relação entre a segurança e o custo.

Para iniciar a demonstração disto, serão explicados os métodos de geração de carregamentos estacionários e não estacionários, estes ultimos desenvolvido neste trabalho, e no fim, ambos serão comparados a um carregamento real, e através de analise estatística, será estimada a similaridade deles com o real. 


\subsection{GERAÇÃO DE AÇÕES ESTACIONÁRIAS}

Este método tem como princípio, o método de Monte Carlo, o qual é um processo de solução aproximada de problemas físicos e matemáticos pela simulação de valores aleatórios. Franco (1993), a partir deste, desenvolveu um método de geração de ações para ventos, chamando-o de método do vento sintético, onde a aleatoriedade de um carregamento seria gerada com base em um espectro de potência de valor inicial nulo, gerando uma média nula, e consequentemente um espectro de valor de área equivalente ao desvio padrão. No entanto, o método do vento sintético pode ser modificado para outras naturezas de carregamento, como sismos, proposto por Corbani (2006), desde que seja aceita à condição de estacionaridade.

O método de geração de ações inicia-se com a escolha de um espectro de potência de valor inicial nulo, então, faz-se a divisão do espectro de potência em n intervalos, devido ao valor da integração nestes intervalos são obtidos coeficientes chamados de harmônicos. Adota-se então uma quantidade de n harmônicos:

$\mathrm{C}^{\mathrm{F}}{ }_{\mathrm{i}}=\sqrt{2 \int_{\omega^{\mathrm{F}-\mathrm{i}}}^{\omega_{\mathrm{i}}^{\mathrm{F+}} \mathrm{S}_{\mathrm{z}}} \mathrm{S}_{\mathrm{z}}(\omega) \mathrm{d} \omega} \quad$ para $\quad \mathrm{i}=1,2, \ldots, \mathrm{n}$

Onde, no modelo clássico os domínios são definidos por:

$$
\begin{aligned}
& \omega_{\mathrm{i}}^{\mathrm{F}+}=2^{\mathrm{i}+0,5-\mathrm{if}} \omega_{\mathrm{f}} \\
& \omega_{\mathrm{i}}^{\mathrm{F}-}=2^{\mathrm{i}-0,5-\mathrm{if}} \omega_{\mathrm{f}}
\end{aligned}
$$

Sendo as relações (5.2) e (5.3) os limites superior e inferior da integração, diferentemente da ordem dos intervalos definidos por Franco (1993), neste trabalho, é considerado que estes aumentam o intervalo no sentido do crescimento da freqüência (Figura 5.1): 

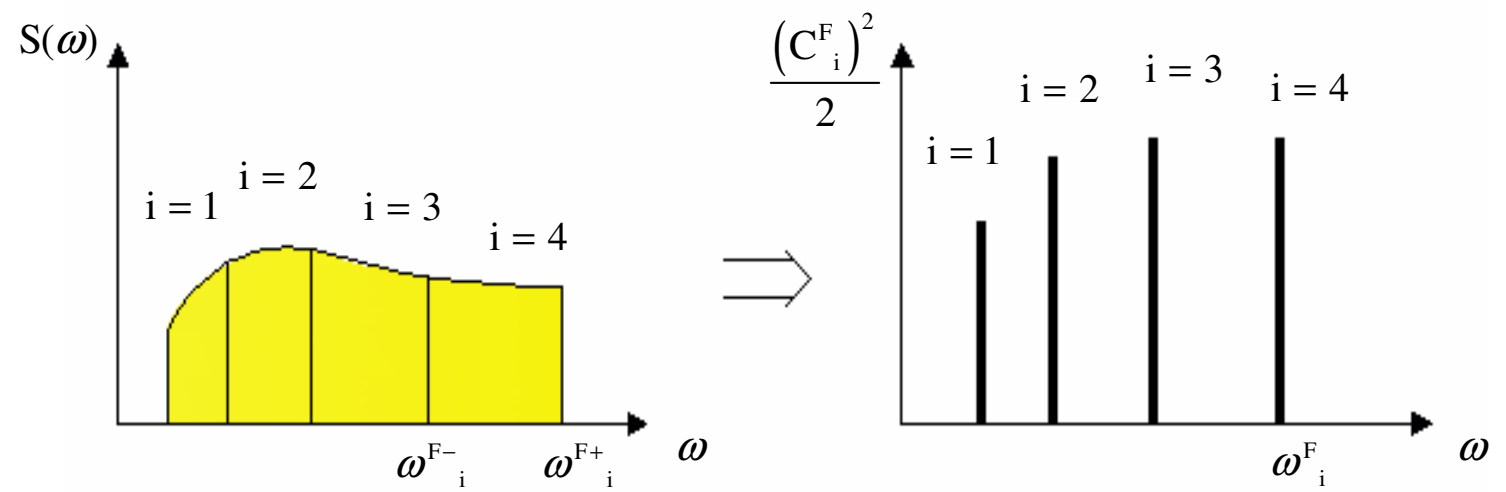

Figura 5.1. Limites de integração da densidade espectral de potência para compor os harmônicos.

Pertencente a estas relações está $\omega_{\mathrm{f}}$, este representa a freqüência angular coincidente com a frequiência fundamental da estrutura, onde o índice if representa o nível que esta ocorre. Quando i = if equivale a dizer que um harmônico contém um valor coincidente com a freqüência fundamental da estrutura, ocasionando um efeito mais desfavorável a estrutura devido à ressonância. Corbani (2006) modificou a taxa de variação do domínio de cada harmônico para:

$$
\begin{aligned}
& \omega_{\mathrm{i}}^{\mathrm{F}+}=\left(\frac{\omega_{\mathrm{f}_{2}}}{\omega_{\mathrm{f}}}\right)^{\mathrm{i}+0,5-\mathrm{if}} \omega_{\mathrm{f}} \\
& \omega_{\mathrm{i}}^{\mathrm{F}-}=\left(\frac{\omega_{\mathrm{f}_{2}}}{\omega_{\mathrm{f}}}\right)^{\mathrm{i}-0,5-\mathrm{if}} \omega_{\mathrm{f}}
\end{aligned}
$$

Onde $\omega_{\mathrm{f}_{2}}$ representa a segunda menor freqüência da estrutura, gerando assim uma ação ainda mais desfavorável, que possua harmônicos coincidentes com as duas menores frequiências da estrutura.

Percebe-se que os harmônicos são valores reais não negativos, não sendo determinadas suas parcelas reais e complexas, devido a isto, são gerados valores pseudo-aleatórios aos ângulos de fase $\phi$, com a intenção de obter os valores complexos para os coeficientes da transformada de Fourier, e por uma questão de simplificação, é utilizada a equação (3.10), devido a isto, chega-se: 
$\mathrm{x}(\mathrm{t})=\sum_{\mathrm{i}=1}^{\mathrm{n}} \mathrm{C}_{\mathrm{i}}^{\mathrm{F}} \cos \left(\omega^{\mathrm{F}} \mathrm{t}-\phi_{\mathrm{i}}\right)$

Onde, no modelo clássico:

$\omega^{\mathrm{F}}=2^{\mathrm{i}-\mathrm{if}} \omega_{\mathrm{f}}$

E no modelo desenvolvido por Corbani (2006):

$\omega_{\mathrm{i}}^{\mathrm{F}}=\left(\frac{\omega_{\mathrm{f}_{2}}}{\omega_{\mathrm{f}}}\right)^{\mathrm{i}-\mathrm{if}} \omega_{\mathrm{f}}$

Percebe-se, quanto ao intervalo de integração para a obtenção dos harmônicos, que estes podem ser determinados de acordo com a necessidade da análise, devido a isto, neste trabalho são determinados dois tipos de intervalos de integração baseados nos intervalos das janelas tempo-freqüência das wavelets harmônicas descritas em (4.131) e (4.141), sendo o intervalo equivalente à janela de variação geométrica definido como:

$$
\begin{array}{lll}
\Delta \operatorname{Ig}_{\mathrm{i}}=\mathrm{q}^{\mathrm{i}-1} & \text { para } & \mathrm{q} \in \mathrm{R}_{+}-[1,0] \\
\operatorname{Ig}_{\mathrm{i}+1}=\operatorname{Ig}_{\mathrm{i}}+\Delta \mathrm{Ig}_{\mathrm{i}} & \text { para } & \mathrm{i} \in \mathrm{N} \\
\operatorname{Ig}_{\mathrm{i}}=1 & \text { para } & \mathrm{i}=1 \\
\omega^{\mathrm{F}}=\frac{2 \omega_{\mathrm{f}}}{\operatorname{Ig}_{\mathrm{i}}+\operatorname{Ig}_{\mathrm{i}+1}} & \text { para } & \mathrm{i}=\mathrm{if} \\
\omega^{\mathrm{F}+}{ }_{\mathrm{i}}=\omega_{0}^{\mathrm{F}}{ }_{0} \operatorname{Ig}_{\mathrm{i}+1} & & \\
\omega^{\mathrm{F}-}{ }_{\mathrm{i}}=\omega_{0}^{\mathrm{F}} \mathrm{Ig}_{\mathrm{i}} & &
\end{array}
$$

E o equivalente a janela de variação aritmética:

$$
\begin{array}{lll}
\Delta \mathrm{Ia}_{\mathrm{i}}=\mathrm{pi} & \text { para } & \mathrm{p} \in \mathrm{R}^{*} \\
\mathrm{Ia}_{\mathrm{i}+1}=\mathrm{Ia}_{\mathrm{i}}+\Delta \mathrm{Ia}_{\mathrm{i}} & \text { para } & \mathrm{i} \in \mathrm{N} \\
\mathrm{Ia}_{\mathrm{i}}=1 & \text { para } & \mathrm{i}=1 \\
\omega^{\mathrm{F}}=\frac{2 \omega_{\mathrm{f}}}{\mathrm{Ia}_{\mathrm{i}}+\mathrm{Ia}_{\mathrm{i}+1}} & \text { para } & \mathrm{i}=\mathrm{if} \\
\omega^{\mathrm{F}+}{ }_{\mathrm{i}}=\omega_{0}^{\mathrm{F}}{ }_{0} \mathrm{Ia}_{\mathrm{i}+1} & & \\
\omega^{\mathrm{F}-}=\omega^{\mathrm{F}}{ }_{0} \mathrm{Ia}_{\mathrm{i}} & &
\end{array}
$$


Onde as equações (5.13), (5.19) representam os limites superiores e (5.14) e (5.20) representam os limites inferiores do intervalo de integração, os valores de (5.12) e (5.18) representam o menor valor de freqüência da ação, para que a freqüência de um determinado harmônico coincida com a freqüência fundamental da estrutura, estes valores também podem ser usados para:

$$
\begin{aligned}
& \omega_{i}^{\mathrm{F}}=\frac{\omega_{0}^{\mathrm{F}}\left(\operatorname{Ig}_{\mathrm{i}}+\operatorname{Ig}_{\mathrm{i}+1}\right)}{2} \\
& \omega_{\mathrm{i}}^{\mathrm{F}}=\frac{\omega_{0}^{\mathrm{F}}\left(\mathrm{Ia}_{\mathrm{i}}+\mathrm{Ia}_{\mathrm{i}+1}\right)}{2}
\end{aligned}
$$

Sendo as equações (5.21) e (5.22) as freqüências dos harmônicos com intervalos de integração de variação geométrica e aritmética, respectivamente. Nota-se que os valores de $\mathrm{p}$ e $\mathrm{q}$ não precisam mais ter valores inteiros, pois como o modelo tem comportamento estacionário, o conceito de janela tempo-freqüência não é utilizado, além disso, os intervalos de integração do modelo clássico e do modelo que leva em conta as duas menores frequiências da estrutura são casos particulares do modelo com variação geométrica, simplesmente considerando:

$\mathrm{q}=2$

$\mathrm{q}=\frac{\omega_{\mathrm{f}_{2}}}{\omega_{\mathrm{f}}}$

Por ultimo, referente às amplitudes da ação, muitas vezes é preferível que o valor máximo do carregamento seja um valor unitário, devido a isto, os valores de (5.1) podem ser normalizados, esta normalização é dada por:

$$
\mathrm{Cn}^{\mathrm{F}}{ }_{\mathrm{i}}=\frac{\mathrm{C}^{\mathrm{F}}{ }_{\mathrm{i}}}{\sum_{\mathrm{i}=1}^{\mathrm{n}} \mathrm{C}^{\mathrm{F}}{ }_{\mathrm{i}}}
$$

O que resulta, a partir de (5.6) em:

$$
\mathrm{xn}(\mathrm{t})=\sum_{\mathrm{i}=1}^{\mathrm{n}} \mathrm{Cn}_{\mathrm{i}}^{\mathrm{F}} \cos \left(\omega_{\mathrm{i}}^{\mathrm{F}} \mathrm{t}-\phi_{\mathrm{i}}\right)
$$


Percebe-se que todas as ações geradas por este método possuem valor médio nulo, o valor médio é então obtido estatisticamente, assim como o valor de pico, este último, quando a ação gerada for normalizada. 


\subsection{GERAÇÃO DE AÇÕES NÃO ESTACIONÁRIAS}

Igualmente as ações estacionárias, são gerados valores aleatórios de ações de média nula, diferentemente destas últimas, para a sua determinação é necessária a utilização de espectros que analizam a variação de energia em relação a sua freqüência e tempo, estes espectros poderiam ser obtidos através do uso de transformadas de wavelets harmônicas para um grupo de sinais, e através dos escalogramas destes sinais, seria possível um estudo estatístico com a intenção de obter uma superfície dependente de determinados parâmetros, assim como os espectros de potência de Davenport para análise de vento e Kanai-Tajimi para sismos (embora este último seja semi-empírico), com a diferença que estes dois são curvas obtidas para sinais estacionários.

No entanto, esta análise seria extremamente extensa. Uma forma de obtenção mais simples de espectrogramas, embora menos precisa, a qual é usada neste trabalho, tem a idéia inicial desenvolvida por Spanos; Tezcan e Tratskas (2005) onde através de espectros de potência que resultam em sinais estacionários como o de Kanai-Tajimi, é possível obter um espectrograma com o uso de uma função de variação ao longo do tempo, e pelo fato do espectro de potência ser em função da freqüência, juntamente com esta função dependente do tempo, um espectrograma é obtido.

Segundo Newland (1993), é possível a simplificação da análise de um sinal não estacionário quando se conhece a natureza física do sinal, ou quando a análise da amostra de sinais permite que o processo não estacionário seja dividido em componentes, onde a primeira se comporta como um processo estacionário e a segunda como uma função determinística de variação ao longo do tempo, neste caso é possível determinar um sinal não estacionário com uma precisão aceitável. Então devido a isto, tem-se:

$x(t)=f(t) z(t)$

Onde $\mathrm{z}(\mathrm{t})$ representa um sinal de comportamento estacionário e $\mathrm{f}(\mathrm{t})$ representa a função de variação ao longo do tempo, podendo ser chamada de função de tendência. 
Segundo Spanos; Tezcan e Tratskas (2005), esta função $f(t)$ em conjunto com um espectro de potência estacionário, o qual pode ser gerado pelo sinal $z(t)$, gera um espectrograma chamado de espectro evolutivo separável (separable evolutionary spectrum), que usualmente pode ser representado pela equação:

$S(\omega, t)=S_{z}(\omega) f(t)^{2}$

Sendo $\mathrm{S}_{\mathrm{z}}(\omega)$ a densidade espectral de potência de comportamento estacionário de $\mathrm{z}(\mathrm{t})$, o qual possui o quadrado da dimensão de $\mathrm{z}(\mathrm{t})$ por unidade de freqüência angular, e $\mathrm{f}(\mathrm{t})^{2}$ decorrente do fato da densidade espectral de potência fornecer o valor quadrado da amplitude do sinal em uma determinada freqüência. Neste trabalho, para se evitar o uso de uma constante dimensional, $\mathrm{f}(\mathrm{t})$ e seu domínio $\mathrm{t}$, que representa o tempo, são considerados adimensionais, ambos com valores que variam de 0 a 1 .

Nota-se, até então, que tanto a densidade espectral de potência $S_{z}(\omega)$, quanto à função $\mathrm{f}(\mathrm{t})$, pertencem ao mesmo sinal, mas para a geração de um sinal não estacionário qualquer, esta condição não é obrigatória, pois, a partir de uma densidade espectral de potência de um carregamento estacionário como o de Davenport ou Kanai-Tajimi podese impor através de uma função qualquer $\mathrm{f}(\mathrm{t})$, um comportamento não estacionário.

Uma forma de conseguir $\mathrm{f}(\mathrm{t})$ é através de um grupo de sinais, onde se podem obter amplitudes máximas em intervalos de tempo, de forma que representem a sua tendência, e através de algum método de ajuste de curva, como o método dos quadrados mínimos, estes pontos se tornam uma curva em função do tempo, sendo esta curva a função de tendência $f(t)$, onde, como explicado anteriormente, ajusta-se para uma extensão e valor máximo unitário.

O espectrograma gerado por (5.28), embora definido a densidade espectral e a função temporal, ainda não pode ser utilizado para encontrar possíveis coeficientes das wavelets harmônicas, pois este espectrograma possui uma superfície contínua (Figura 5.2 (a) e 5.2 (b)): 
(a)

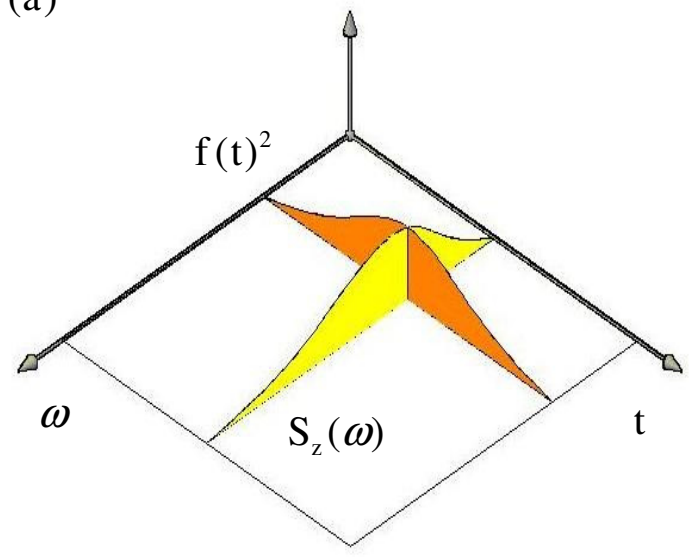

(b)

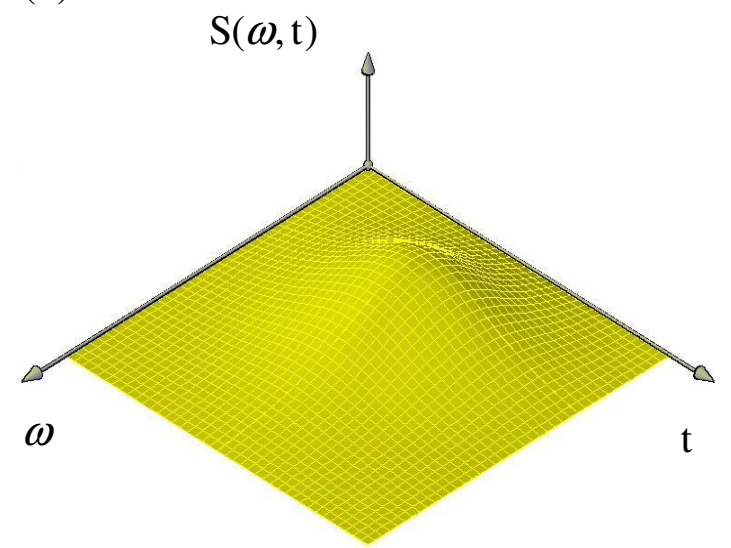

Figura 5.2. Construção do espectograma: (a) Função de tendência ao quadrado e espectro de potência com comportamento estacionário; (b) A junção das duas funções gera o espectro de potência não estacionário.

Então se faz necessária à determinação do domínio da freqüência e do tempo para, através de um processo de integração, representar os coeficientes das wavelets harmônicas. Então partindo do teorema de Parseval e utilizando (5.28), chega-se:

$\frac{1}{\mathrm{~T}} \int_{0}^{\mathrm{T}} \mathrm{x}(\mathrm{t})^{2} \mathrm{dt}=\int_{\omega} \int_{\mathrm{t}} \mathrm{S}(\omega, \mathrm{t}) \mathrm{dtd} \omega=\int_{\omega} \int_{\mathrm{t}} \mathrm{S}_{\mathrm{z}}(\omega) \mathrm{f}(\mathrm{t})^{2} \mathrm{dtd} \omega$

Percebe-se que em (5.29), a freqüência e o tempo ocorrem em funções independentes, devido a este fator, podem ser integrados de uma forma separada. A integração no domínio da freqüência é dividida em n partes, onde cada parte será utilizada para um nível do escalograma, estas partes podem ser obtidas a partir de espectros de freqüência simétricos e não negativos, pois os carregamentos em estruturas possuem valores exclusivamente reais, gerando assim simetria no espectro.

Neste trabalho, serão usadas as variações de janelas tempo-freqüência descritas nas equações (4.131) e (4.141), e com o intuito da redução das equações explicitadas, são definidas as seguintes relações:

$$
\begin{aligned}
& \alpha_{\mathrm{j}}=\operatorname{Pg}_{\mathrm{j}} \quad \text { ou } \quad \alpha_{\mathrm{j}}=\mathrm{Pa}_{\mathrm{j}} \\
& \Delta \alpha_{\mathrm{j}}=\Delta \mathrm{Pg}_{\mathrm{j}} \text { ou } \Delta \alpha_{\mathrm{j}}=\Delta \mathrm{Pa}_{\mathrm{j}}
\end{aligned}
$$


Sendo os valores de $\alpha_{\mathrm{j}}$ e $\Delta \alpha_{\mathrm{j}}$ válidos para as variações em escala geométrica e aritmética. A partir destas considerações, faz-se:

$$
\begin{aligned}
& I_{j}=\int_{\omega^{w-}{ }_{j}{ }_{j}{ }^{w+}{ }_{j}} S_{z}(\omega) d \omega \quad \text { para } j=0,1, \ldots,(n-2) \\
& I S_{n-1}=\int_{\omega^{w-}{ }_{n-1}}^{\omega^{w+}{ }_{n-1}} S_{z}(\omega) d \omega
\end{aligned}
$$

Onde, para (5.32) com intervalos de integração com variação geométrica ou aritmética, tem-se:

$$
\begin{aligned}
& \omega^{\mathrm{w}}{ }_{0}=\frac{2 \omega_{\mathrm{f}}}{\alpha_{\mathrm{j}}+\alpha_{\mathrm{j}+1}} \quad \text { para } \quad \mathrm{j}=\mathrm{jf} \\
& \omega^{\mathrm{w}+}{ }_{\mathrm{j}}=\omega^{\mathrm{w}}{ }_{0} \alpha_{\mathrm{j}+1} \\
& \omega^{\mathrm{w}-}{ }_{\mathrm{j}}=\omega^{\mathrm{w}}{ }_{0} \alpha_{\mathrm{j}}
\end{aligned}
$$

E para (5.33) os intervalos de integração são definidos como:

$$
\begin{aligned}
& \omega^{\mathrm{w}+}{ }_{\mathrm{n}-1}=\omega^{\mathrm{w}+}{ }_{\mathrm{n}-2}+\omega^{\mathrm{w}}{ }_{0} \\
& \omega^{\mathrm{w}-}{ }_{\mathrm{n}-1}=\omega^{\mathrm{w}+}{ }_{\mathrm{n}-2}
\end{aligned}
$$

Equivalente ao processo do vento sintético, descrito por Franco (1993), a relação (5.35), é o limite superior e (5.36) representa o limite inferior das integrações dos primeiros n-1 intervalos, estes aumentam de valor no sentido do crescimento da freqüência, onde os limites equivalem aos níveis do escalogama, sendo cada nível referenciado por j (Figura 5.3). O intervalo do último nível, com os limites representados por (5.37) e (5.38) é igual ao primeiro intervalo, isto ocorre pelo fato da janela tempo-freqüência ser a mesma 

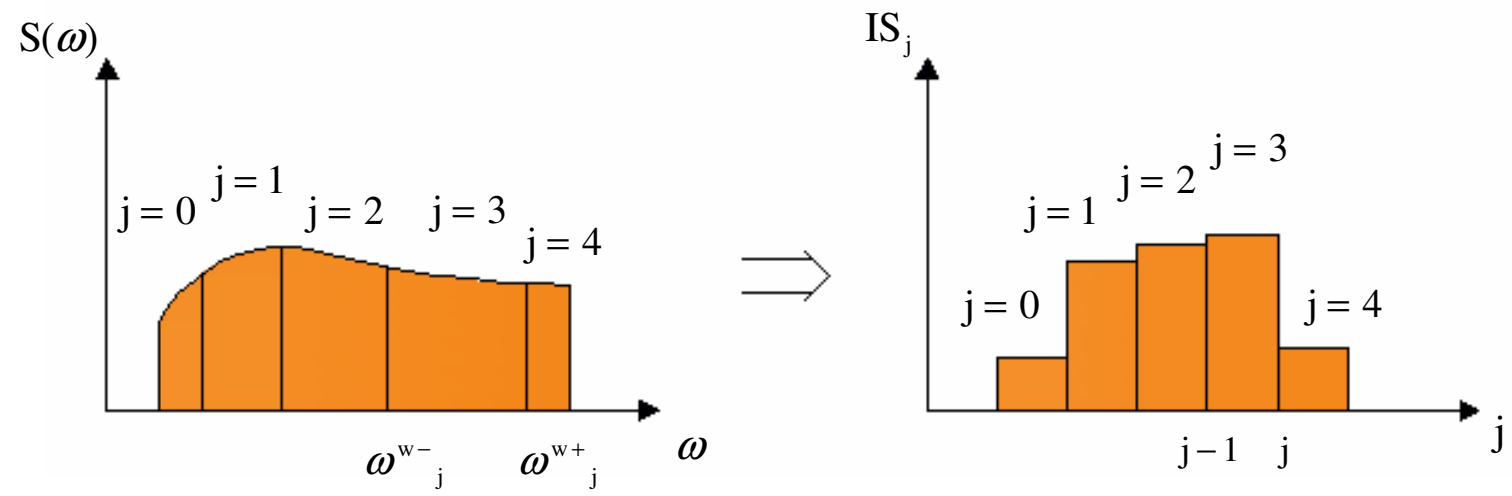

Figura 5.3. Limites de integração da densidade espectral de potência para compor os coeficientes da transformada da wavelet harmônica.

Como pode ser observado, o nível que representa a função de escala (j=-1), não esta presente, pois este possui valor nulo, devido ao fato do carregamento gerado possuir média nula.

Pertencente aos limites apresentados está $\omega_{\mathrm{f}}$, este representa a freqüência angular coincidente com a frequiência fundamental da estrutura, onde o índice jf representa o nível que esta ocorre. Quando $\mathrm{j}=\mathrm{jf}$ equivale a dizer que um nível do escalograma contém um valor coincidente com a freqüência fundamental da estrutura, ocasionando um efeito mais desfavorável a estrutura devido à ressonância.

Para a integração em função do tempo, é obtida a potência média da função de tendência, esta integração pode ser separada em várias partes para cada nível, com a intenção de compor os coeficientes das wavelets harmônicas existente no mesmo. Devido ao fato do período $\mathrm{T}$ ser unitário, cada parcela de um mesmo nível terá um período de $1 / \Delta \alpha_{\mathrm{j}}$, então, faz-se:

$$
\begin{aligned}
& \overline{\mathrm{f}}_{\alpha_{\mathrm{j}}+\mathrm{k}}^{2}=\Delta \alpha_{\mathrm{j}} \int_{\mathrm{t}_{\alpha_{\mathrm{j}}+\mathrm{k}}^{-}}^{\mathrm{t}_{\alpha_{\mathrm{j}}+\mathrm{k}}^{+}} \mathrm{f}(\mathrm{t})^{2} \mathrm{dt} \quad \text { para } \mathrm{j}=0,1, \ldots,(\mathrm{n}-2) \\
& \overline{\mathrm{f}}_{\mathrm{N} / 2}^{2}=\int_{0}^{1} \mathrm{f}(\mathrm{t})^{2} \mathrm{dt}
\end{aligned}
$$


Onde:

$\begin{array}{lll}\mathrm{t}_{\alpha_{\mathrm{j}}+\mathrm{k}}^{+}=\frac{\mathrm{k}+1}{\Delta \alpha_{\mathrm{j}}} & \text { para } & \mathrm{k}=0,1, \ldots,\left(\Delta \alpha_{\mathrm{j}}-1\right) \\ \mathrm{t}_{\alpha_{\mathrm{j}}+\mathrm{k}}^{-}=\frac{\mathrm{k}}{\Delta \alpha_{\mathrm{j}}} & \text { para } & \mathrm{k}=0,1, \ldots,\left(\Delta \alpha_{\mathrm{j}}-1\right)\end{array}$

O valor de (5.39) representa a potência média da função de tendência nos intervalos especificados em (5.41) e (5.42). A partir destes resultados, os coeficientes das wavelets harmônicas podem ser encontrados, pois este é equivalente ao valor da soma dos quadrados dos módulos de dois coeficientes das wavelets harmônicas, sendo isto feito para todos os domínios, o escalograma pode ser utilizado.

$$
\begin{aligned}
& \left|\mathrm{w}_{\alpha_{\mathrm{j}}+\mathrm{k}}\right|^{2}+\left|\overline{\mathrm{w}}_{\alpha_{\mathrm{j}}+\mathrm{k}}\right|^{2}=\mathrm{IS}_{\mathrm{j}} \overline{\mathrm{f}}_{\alpha_{\mathrm{j}}+\mathrm{k}}^{2} \quad \text { para } \quad \mathrm{j}=0,1, \ldots,(\mathrm{n}-2) \\
& \left|\mathrm{w}_{\mathrm{N} / 2}\right|^{2}=\mathrm{IS}_{\mathrm{n}-1} \overline{\mathrm{f}}_{\mathrm{N} / 2}^{2}
\end{aligned}
$$

Como o sinal que se pretende gerar é real, o modulo dos conjugados são iguais, (5.43) então torna-se:

$$
\begin{aligned}
& \left|\mathrm{w}_{\alpha_{\mathrm{j}}+\mathrm{k}}\right|=\left|\overline{\mathrm{w}}_{\alpha_{\mathrm{j}}+\mathrm{k}}\right|=\sqrt{\frac{\mathrm{IS}_{\mathrm{j}} \overline{\mathrm{f}}_{\alpha_{\mathrm{j}}+\mathrm{k}}^{2}}{2}} \quad \text { para } \mathrm{j}=0,1, \ldots,(\mathrm{n}-2) \\
& \left|\mathrm{w}_{\mathrm{N} / 2}\right|=\sqrt{\mathrm{IS} \mathrm{S}_{\mathrm{n}-1} \overline{\mathrm{f}}_{\mathrm{N} / 2}^{2}}
\end{aligned}
$$

Embora a forma do escalograma seja modificada, sua energia permanece a mesma, este procedimento pode ser utilizado com diversos espectros de potência e com diversas funções de tendência. Percebe-se que os coeficientes estão em módulos, não sendo determinadas suas parcelas reais e complexas, devido a isto, são gerados valores pseudo-aleatórios aos ângulos de fase $\phi$, com a intenção de obter os valores complexos para os coeficientes das wavelets harmônicas devido a um sinal de valores reais:

$$
\begin{aligned}
& \mathrm{w}_{\alpha_{\mathrm{j}}+\mathrm{k}}=\left|\mathrm{w}_{\alpha_{\mathrm{j}}+\mathrm{k}}\right| \mathrm{e}^{-i \phi_{\alpha_{\mathrm{j}}+\mathrm{k}}} \quad \text { para } \quad \mathrm{j}=0,1, \ldots,(\mathrm{n}-2) \\
& \overline{\mathrm{w}}_{\alpha_{\mathrm{j}}+\mathrm{k}}=\left|\overline{\mathrm{w}}_{\alpha_{\mathrm{j}}+\mathrm{k}}\right| \mathrm{e}^{i \phi_{\alpha_{\mathrm{j}}+\mathrm{k}}} \quad \text { para } \quad \mathrm{j}=0,1, \ldots,(\mathrm{n}-2) \\
& \mathrm{w}_{\mathrm{N} / 2}= \pm\left|\mathrm{w}_{\mathrm{N} / 2}\right|
\end{aligned}
$$


Com os coeficientes das wavelets harmônicas conseguidas através de (5.47) a (5.49), é possível encontrar os coeficientes da transformada de Fourier, através do uso, respectivamente, das equações (4.167), (4.168), (4.169), (4.170), e (4.108) mostradas a seguir:

$$
\begin{array}{lll}
w_{\alpha_{\mathrm{j}}+\mathrm{k}}=\mathrm{w}_{\alpha_{\mathrm{j}} \mathrm{k}} \mathrm{e}^{-\mathrm{i} 2 \pi \alpha_{\mathrm{j}} \mathrm{k} / \Delta \alpha_{\mathrm{j}}} & \text { para } & \mathrm{k}=0,1, \ldots,\left(\Delta \alpha_{\mathrm{j}}-1\right) \\
\bar{w}_{\alpha_{\mathrm{j}}+\mathrm{k}}=\overline{\mathrm{w}}_{\alpha_{\mathrm{j}}+\mathrm{k}} \mathrm{e}^{\mathrm{i} 2 \pi \alpha_{\mathrm{j}} \mathrm{k} / \Delta \alpha_{\mathrm{j}}} & \text { para } & \mathrm{k}=0,1, \ldots,\left(\Delta \alpha_{\mathrm{j}}-1\right) \\
\mathrm{X}_{\alpha_{\mathrm{j}}+\mathrm{r}}=\frac{1}{\Delta \alpha_{\mathrm{j}}} \sum_{\mathrm{k}=0}^{\Delta \alpha_{\mathrm{j}}-1} w_{\alpha_{\mathrm{j}} \mathrm{k}} \mathrm{e}^{-i 2 \pi \mathrm{r} / \Delta \alpha_{\mathrm{j}}} & \text { para } & \mathrm{r}=0,1, \ldots,\left(\Delta \alpha_{\mathrm{j}}-1\right) \\
\overline{\mathrm{X}}_{\alpha_{\mathrm{j}}+\mathrm{r}}=\frac{1}{\Delta \alpha_{\mathrm{j}}} \sum_{\mathrm{k}=0}^{\Delta \alpha_{\mathrm{j}}-1} \bar{w}_{\alpha_{\mathrm{j}}+\mathrm{k}} \mathrm{e}^{i 2 \pi \mathrm{rk} / \Delta \alpha_{\mathrm{j}}} & \text { para } & \mathrm{r}=0,1, \ldots,\left(\Delta \alpha_{\mathrm{j}}-1\right) \\
\mathrm{X}_{\mathrm{N} / 2}=\mathrm{w}_{\mathrm{N} / 2} & &
\end{array}
$$

Feito isto, com o uso de uma IDFT simples, é possível obter um carregamento de comportamento não estacionário, gerado de forma aleatória. Então, em posse dos coeficientes da transformada de Fourier, é possível obter o sinal, através da aplicação da IDFT como em (3.67):

$$
\mathrm{X}_{\mathrm{r}}=\sum_{\mathrm{k}=0}^{\mathrm{N}-1} \mathrm{X}_{\mathrm{k}} \mathrm{e}^{i(2 \pi \mathrm{kr} / \mathrm{N})} \quad \mathrm{para} \quad \mathrm{r}=0,1, \ldots,\left(2^{\mathrm{n}}-1\right)
$$

Todo este processo, a partir da obtenção dos coeficientes das wavelets harmônicas até a geração do carregamento é equivalente a IDHWT. A aplicação da FFT em (5.52), (5.53) e (5.54) é possível com a inclusão de zeros para o processamento e exclusão destes para a resposta. Quando se utiliza pontos em quantidade equivalente a $\mathrm{q}=2$, a aplicação da FFT se torna mais rápida, pois não é necessário truncamentos ou adição de valores nulos no sinal.

A normalização do sinal através da normalização dos coeficientes das wavelets harmônicas como em (5.25) é feita apenas para o domínio da frequiência, sendo a variável normalizada não (5.32) e (5.33), mas a amplitude, que é obtida de forma similar a (5.1): 
$\mathrm{C}^{\mathrm{w}}{ }_{\mathrm{j}}=\sqrt{2 \mathrm{IS}_{\mathrm{j}}} \quad$ para $\quad \mathrm{j}=0,1, \ldots,(\mathrm{n}-1)$

Então, equivalente a (5.25), a normalização é dada por:

$\mathrm{Cn}^{\mathrm{w}}{ }_{\mathrm{j}}=\frac{\mathrm{C}^{\mathrm{w}}{ }_{\mathrm{j}}}{\sum_{\mathrm{j}=0}^{\mathrm{n}-1} \mathrm{C}^{\mathrm{w}}{ }_{\mathrm{j}}}$

Com a substituição pelas amplitudes normalizadas, faz-se o caminho inverso de (5.56):

$\mathrm{ISn}_{\mathrm{j}}=\frac{\left(\mathrm{Cn}^{\mathrm{w}}{ }_{\mathrm{j}}\right)^{2}}{2} \quad$ para $\quad \mathrm{j}=0,1, \ldots,(\mathrm{n}-1)$

Onde (5.58) é usado nas equações (5.45) e (5.46):

$\left|\mathrm{w}_{\alpha_{\mathrm{j}}+\mathrm{k}}\right|=\left|\overline{\mathrm{w}}_{\alpha_{\mathrm{j}} \mathrm{k} \mathrm{k}}\right|=\sqrt{\frac{\mathrm{ISn}_{\mathrm{j}} \overline{\mathrm{f}}_{\alpha_{\mathrm{j}}+\mathrm{k}}^{2}}{2}} \quad$ para $\mathrm{j}=0,1, \ldots,(\mathrm{n}-2)$
$\left|\mathrm{w}_{\mathrm{N} / 2}\right|=\sqrt{\mathrm{ISn}_{\mathrm{n}-1} \overline{\mathrm{f}}_{\mathrm{N} / 2}^{2}}$

Obtidos estes coeficientes, todas as etapas seguintes para a obtenção da ação normalizada são as mesmas. Percebe-se que todas as ações geradas por este método também possuem valor médio nulo, o valor médio é então obtido estatisticamente, assim como o valor de pico, este último, quando a ação gerada for normalizada. 


\subsection{UTILIZAÇÃO DAS AÇÕES PARA VENTO}

Embora seja usual na natureza que o vento tenha um comportamento próximo do estacionário, nada impede, mediante observação da variação, que seja usada a não estacionaridade para casos específicos, com a intenção de obter uma resposta da estrutura mais próxima da real.

Segundo o método do vento sintético, separa-se o sinal da velocidade do vento em duas parcelas, uma flutuante e uma estática, esta ultima sendo chamada de velocidade média, onde geralmente a razão entre a velocidade média e a velocidade máxima de rajada é em torno da metade da velocidade total, outra característica importante reside no fato do sinal do vento ser representado apenas por números reais, ocasionando em simetria para os espectros, reduzindo o trabalho para a determinação dos coeficientes.

De acordo com Carril Jr (2000), a velocidade média e máxima do vento varia em função principalmente da altitude, e de outros fatores como as características do terreno, adotando assim equações obtidas empiricamente. Em posse destas velocidades, as pressões média e máxima também podem ser definidas através de equações empíricas, estas relações entre altitude, velocidade e pressão variam de acordo a norma de cada pais, no entanto, tem-se:

$$
\begin{aligned}
& \mathrm{V}_{\text {est }}=\hat{\mathrm{V}}_{\text {est }}(\mathrm{z}) \\
& \mathrm{V}_{\text {pic }}=\hat{\mathrm{V}}_{\text {pic }}(\mathrm{z}) \\
& p_{\text {est }}=\hat{p}_{\text {est }}\left(\mathrm{V}_{\text {est }}\right) \\
& p_{\text {pic }}=\hat{p}_{\text {pic }}\left(\mathrm{V}_{\text {pic }}\right)
\end{aligned}
$$

Onde $\mathrm{z}, \mathrm{V}$ e $p$ são respectivamente a altitude, velocidade e pressão do vento medida sobre o terreno, os índices est e pic representam a parcela estática e de pico, com a diferença entre estas, determina-se a parte flutuante das pressões do vento:

$$
p_{\mathrm{f}}=p_{\text {pic }}-p_{\text {est }}
$$


Sendo a parte flutuante a representação da maior variação de pressão possível no carregamento. As densidades espectrais de potência utilizadas para estudo de vento geralmente, também possuem valores que dependem da velocidade do vento em determinada altitude, mas algumas densidades espectrais são definidas com uma altitude fixada em 10 metros. Um exemplo disto é o espectro de potência do vento de Davenport:

$$
\frac{\mathrm{W}_{\mathrm{v}}(f)}{\sigma_{\mathrm{v}}{ }^{2}}=\frac{4 \mathrm{v}^{2}}{f\left(1+\mathrm{v}^{2}\right)^{\frac{4}{3}}} \quad \text { para } \quad \mathrm{v}=\frac{1200 f}{\mathrm{~V}}
$$

Em que $f$ é a frequiência das rajadas, $\mathrm{V}$ é a velocidade média do vento a uma altitude de 10 metros e $\sigma_{\mathrm{v}}{ }^{2}$ é o desvio padrão da velocidade do vento, pois o espectro $\mathrm{W}_{\mathrm{v}}(f)$ dividido pelo desvio padrão gera um valor de dimensão pertencente ao inverso da freqüência linear $(1 / f)$. O espectro de potência do vento para um comportamento estacionário adotado neste trabalho é o da norma canadense, resultante de modificações do espectro de Davenport, definido por:

$$
\frac{\mathrm{W}_{\mathrm{v}}(f)}{\sigma_{\mathrm{v}}{ }^{2}}=\frac{4 \mathrm{v}^{2}}{f\left(1+\mathrm{v}^{2}\right)^{\frac{4}{3}}} \quad \text { para } \quad \mathrm{v}=\frac{1220 f}{\mathrm{~V}}
$$

A componente flutuante da pressão do vento pode ser representada, de acordo com Franco (1993), aproximadamente, por um número finito de funções harmônicas convenientemente escolhidas com períodos entre 600 e 0,5 segundos, equivalente a freqüências de 0,0017 e $2 \mathrm{~Hz}$, de modo a abranger pelo menos o primeiro modo de vibração da estrutura. Adota-se então uma quantidade de $n$ harmônicos, os quais um contenha a freqüência $f_{\mathrm{f}}$, coincidente com o primeiro modo de vibração da estrutura. Devido a isto, a partir de (5.1), tem-se:

$$
\mathrm{C}^{\mathrm{F}}{ }_{\mathrm{i}}=\sqrt{2 \int_{f_{\mathrm{i}}^{-}}^{f_{\mathrm{i}}^{+}} \frac{\mathrm{W}_{\mathrm{v}}(f)}{\sigma_{\mathrm{v}}{ }^{2}} \mathrm{~d} f} \quad \text { para } \quad \mathrm{i}=1,2, \ldots, \mathrm{n}
$$


E para os limites de integração, utilizando os valores de (5.13) e (5.14) ou (5.19) e (5.20), chega-se:

$$
\begin{aligned}
f^{\mathrm{F}+}{ }_{\mathrm{i}} & =\frac{\omega^{\mathrm{F}+}{ }_{\mathrm{i}}}{2 \pi} \\
f^{\mathrm{F}-}{ }_{\mathrm{i}} & =\frac{\omega^{\mathrm{F}-}{ }_{\mathrm{i}}}{2 \pi}
\end{aligned}
$$

Percebe-se, neste caso, que o coeficiente do harmônico é adimensional, pois o domínio de integração é a frequiência linear. Quando se pretende gerar um comportamento não estacionário, a variável tempo é necessária, esta é introduzida através da função de tendência, que a partir da relação (5.28), tem-se:

$\mathrm{W}(f, \mathrm{t})=\frac{\mathrm{W}_{\mathrm{v}}(f)}{\sigma_{\mathrm{v}}{ }^{2}} \mathrm{f}(\mathrm{t})^{2}$

Então baseado em (5.32), chega-se:

$\mathrm{IS}_{\mathrm{j}}=\int_{f^{\mathrm{w}-}{ }_{\mathrm{j}}}^{f_{\mathrm{w}+}^{\mathrm{w}}} \frac{\mathrm{W}_{\mathrm{v}}(\mathrm{f})}{\sigma_{\mathrm{v}}{ }^{2}} \mathrm{~d} f \quad$ para $\quad \mathrm{j}=0,1, \ldots,(\mathrm{n}-1)$

E para os limites de integração, utilizando os valores de (5.35) e (5.36) ou (5.37) e (5.38) para o último nível, chega-se:

$$
\begin{aligned}
f^{\mathrm{w}+}{ }_{\mathrm{j}} & =\frac{\omega^{\mathrm{w}+}{ }_{\mathrm{j}}}{2 \pi} \\
f^{\mathrm{w}-}{ }_{\mathrm{j}} & =\frac{\omega^{\mathrm{w}-}{ }_{\mathrm{j}}}{2 \pi}
\end{aligned}
$$

Para a aplicação do carregamento devido ao vento na estrutura, inicialmente divide-se esta última em regiões chamadas módulos, onde cada módulo recebe este carregamento com uma determinada intensidade, correspondendo a uma simulação de rajada. Para definir estas intensidades, em um carregamento estacionário, Franco (1993) definiu a seguinte correlação espacial para pressões de vento:

$\mathrm{Cr}\left(f_{\mathrm{i}}\right)=\mathrm{e}^{-\left(\frac{2 f_{\mathrm{i}} \sqrt{\mathrm{C}_{\mathrm{z}}{ }^{2} \Delta \mathrm{z}^{2}+\mathrm{C}_{\mathrm{y}}{ }^{2} \Delta \mathrm{y}^{2}}}{\mathrm{~V}}\right)}$

$$
\text { para } \quad \mathrm{i}=1,2, \ldots, \mathrm{n}
$$


Onde $\Delta \mathrm{ze} \Delta \mathrm{y}$ são as distâncias na vertical e horizontal do centro de rajada até um determinado ponto da estrutura, e os coeficientes $\mathrm{C}_{\mathrm{z}}$ e $\mathrm{C}_{\mathrm{y}}$ são obtidos experimentalmente, e que segundo Franco (1993), por questão de segurança, recebem os valores, respectivamente, de 7 e 12. Por ultimo, o valor da frequiência linear $f$ dos harmônicos, podendo ser baseada em (5.21) ou (5.22) é obtida pela equação:

$$
f_{i}^{\mathrm{F}}=\frac{\omega_{i}^{\mathrm{F}}}{2 \pi}
$$

Quando uma estrutura tiver uma das dimensões muito maior que a outra, como uma torre ou ponte, a dimensão menor perde importância, então a equação (5.75) pode ser simplificada para:

$$
\begin{aligned}
& \operatorname{Crz}\left(f^{\mathrm{F}}{ }_{\mathrm{i}}\right)=\mathrm{e}^{-\left(\frac{2 f_{\mathrm{i}}^{\mathrm{F}} \mathrm{C}_{z} \Delta z}{\mathrm{~V}}\right)} \\
& \operatorname{Cry}\left(f^{\mathrm{F}}{ }_{\mathrm{i}}\right)=\mathrm{e}^{-\left(\frac{2 f_{\mathrm{i}}^{\mathrm{F}} \mathrm{C}_{\mathrm{y}} \Delta \mathrm{y}}{\mathrm{V}}\right)}
\end{aligned}
$$

A função de correlação espacial para pressões de vento, como definida por Franco (1993), possui um formato similar ao de um triângulo, então para cada frequiência do vento, adota-se uma correlação espacial representada por dois triângulos com decaimento de 1 a 0 , devido a isto, para as equações (5.77) e (5.78), chega-se respectivamente ao comprimento total da altura e da largura da rajada para cada harmônico:

$$
\begin{aligned}
& 2 \Delta \mathrm{z}_{\mathrm{i}}=\frac{2 \mathrm{~V}}{\mathrm{C}_{\mathrm{z}} f^{\mathrm{F}}{ }_{\mathrm{i}}} \\
& 2 \Delta \mathrm{y}_{\mathrm{i}}=\frac{2 \mathrm{~V}}{\mathrm{C}_{\mathrm{y}} f^{\mathrm{F}}{ }_{\mathrm{i}}}
\end{aligned}
$$

Onde $\Delta \mathrm{z}$ e $\Delta \mathrm{y}$ são as distâncias a partir do centro da rajada, devido a isto, cada uma é multiplicada por 2. Para aplicar o conceito de tamanho de rajada, segundo Franco (1993), adota-se, a favor da segurança, o centro de rajada na posição mais desfavorável da estrutura onde a resposta desejada seja máxima, sejam para os deslocamento ou esforços solicitantes. 


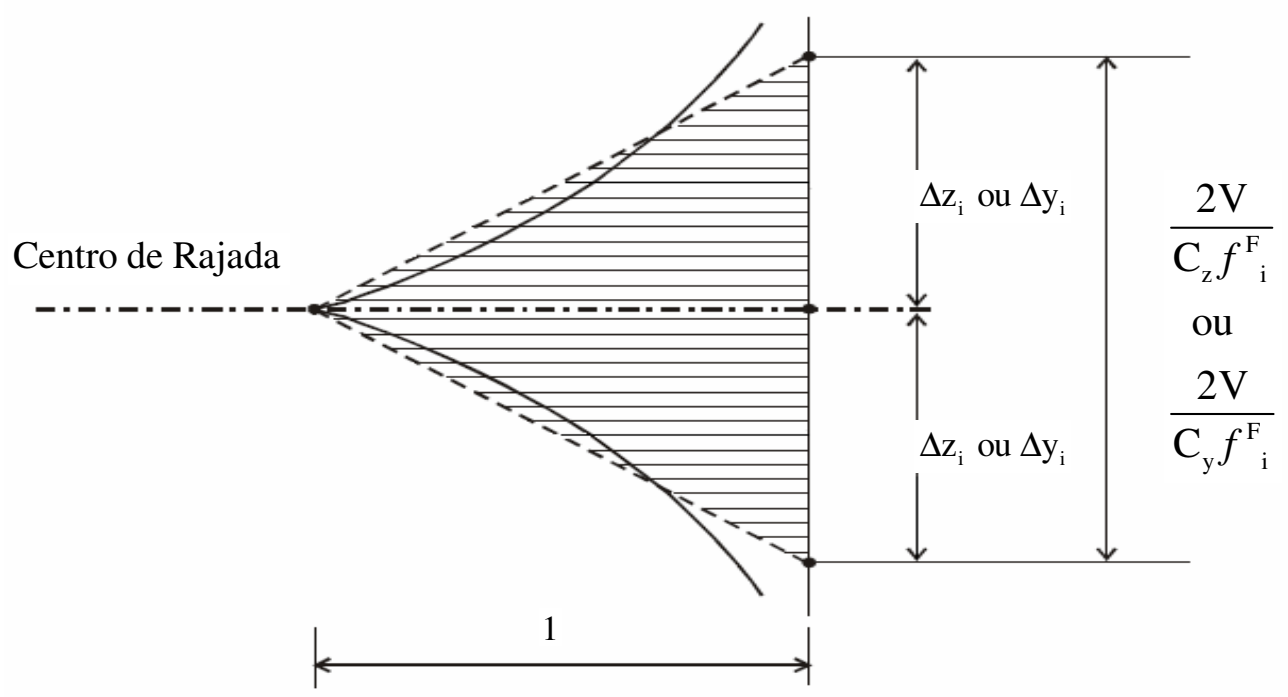

Figura 5.4. Rajada equivalente determinada por Franco (1993).

Depois de estabelecidas as bases destes triângulos por (5.79) e (5.80), e as alturas de valores unitários, pode-se aplicar o valor da correlação da altura e da largura, respectivamente, em cada harmônico:

$\operatorname{Crz}(\mathrm{z})_{\mathrm{i}}=\mid \begin{array}{lll}\frac{1}{\Delta \mathrm{z}_{\mathrm{i}}}(\mathrm{Gcz}-\mathrm{z})+1 & \text { para } & \mathrm{Gcz} \leq \mathrm{z} \leq \mathrm{Gcz}+\Delta \mathrm{z}_{\mathrm{i}} \\ \frac{1}{\Delta \mathrm{z}_{\mathrm{i}}}(\mathrm{z}-\mathrm{Gcz})+1 & \text { para } & \mathrm{Gcz}-\Delta \mathrm{z}_{\mathrm{i}} \leq \mathrm{z} \leq \mathrm{Gcz}\end{array}$

$\operatorname{Cry}(y)_{i}=\mid \begin{array}{lll}\frac{1}{\Delta y_{i}}(\text { Gcy }-y)+1 & \text { para } & \text { Gcy } \leq y \leq \text { Gcy }+\Delta y_{i} \\ \frac{1}{\Delta y_{i}}(y-G c y)+1 & \text { para } & \text { Gcy }-\Delta y_{i} \leq y \leq \text { Gcy }\end{array}$

Sendo Gcz e Gcy a altura e a largura do centro da rajada. Com os valores das correlações espaciais e das pressões flutuantes para cada módulo da estrutura, podem-se encontrar os valores das pressões para cada um deste, pois através da aplicação de (5.65), (5.81) e (5.82) em (5.26), chega-se:

$$
\begin{aligned}
& p(\overline{\mathrm{z}}, \mathrm{t})=p_{\mathrm{f}}(\overline{\mathrm{z}})\left(\sum_{\mathrm{i}=1}^{\mathrm{n}} \operatorname{Crz}(\overline{\mathrm{z}})_{\mathrm{i}} \mathrm{Cn}^{\mathrm{F}}{ }_{\mathrm{i}} \cos \left(\omega^{\mathrm{F}}{ }_{\mathrm{i}} \mathrm{t}-\phi_{\mathrm{i}}\right)\right) \\
& p\left(\mathrm{z}_{\mathrm{Gc}}, \overline{\mathrm{y}}, \mathrm{t}\right)=p_{\mathrm{f}}\left(\mathrm{z}_{\mathrm{Gc}}\right)\left(\sum_{\mathrm{i}=1}^{\mathrm{n}} \operatorname{Cry}(\overline{\mathrm{y}})_{\mathrm{i}} \operatorname{Cn}^{\mathrm{F}}{ }_{\mathrm{i}} \cos \left(\omega^{\mathrm{F}}{ }_{\mathrm{i}} \mathrm{t}-\phi_{\mathrm{i}}\right)\right)
\end{aligned}
$$


Onde $\overline{\mathrm{z}}$ é a coordenada vertical do centro de um modulo de uma estrutura alta, $\bar{y}$ é a coordenada horizontal do centro de um modulo de uma estrutura larga e $\mathrm{z}_{\mathrm{Gc}}$ é a altura do centro de rajada que pode coincidir ou não com $\overline{\mathrm{Z}}$ em (5.83).

Quando for feita a correlação espacial para pressões de vento de um carregamento não estacionário, uma forma de análise é a divisão em intervalos de tempo, para cada intervalo é feita à mesma análise descrita para o comportamento estacionário, analisando quais freqüências pertencem a este, assim como se o centro de rajada permanece no mesmo local.

No entanto, pelo fato das ações devido ao vento terem comportamento próximo do estacionário na maioria dos casos, para a obtenção das pressões do vento para comportamento não estacionário, neste trabalho, são baseadas no modelo estacionário, não sendo considerada, então, a variação do centro de rajada.

Para a modificação do modelo estacionário, é necessária a consideração de uma única freqüência associada um harmônico, este ultimo, no caso das wavelets, a um nível, mas um nível representa um intervalo de freqüências, e devido a isto, é feita a seguinte consideração:

$$
f^{\mathrm{w}}{ }_{\mathrm{j}}=\frac{f_{\mathrm{j}}^{\mathrm{w}-}+f^{\mathrm{w}+}}{2} \quad \text { para } \quad \mathrm{j}=0,1, \ldots,(\mathrm{n}-1)
$$

Sendo, então, possível o uso de todas as relações de (5.79) a (5.82), com a diferença que agora são considerados os níveis e não mais os harmônicos, devido a isto, as freqüências representadas por $f^{\mathrm{F}}{ }_{\mathrm{i}}$ são substituídas por $f^{\mathrm{w}}{ }_{\mathrm{j}}$. Então, a partir da introdução de (5.81) e (5.82) em (5.59) e (5.60), chega-se:

$$
\begin{aligned}
& \left|\mathrm{w}(\overline{\mathrm{z}})_{\alpha_{\mathrm{j}}+\mathrm{k}}\right|=\left|\overline{\mathrm{w}}(\overline{\mathrm{z}})_{\alpha_{\mathrm{j}}+\mathrm{k}}\right|=\sqrt{\frac{\operatorname{Crz}(\overline{\mathrm{z}})_{\mathrm{j}} \operatorname{ISn}_{\mathrm{j}} \overline{\mathrm{f}}_{\alpha_{\mathrm{j}}+\mathrm{k}}^{2}}{2}} \quad \text { para } \quad \mathrm{j}=0,1, \ldots,(\mathrm{n}-2) \\
& \left|\mathrm{w}(\overline{\mathrm{Z}})_{\mathrm{N} / 2}\right|=\sqrt{\operatorname{Crz}(\overline{\mathrm{Z}})_{\mathrm{n}-1} \operatorname{ISn}_{\mathrm{n}-1} \overline{\mathrm{f}}_{\mathrm{N} / 2}^{2}} \\
& \left|\mathrm{w}(\overline{\mathrm{y}})_{\alpha_{\mathrm{j}}+\mathrm{k}}\right|=\left|\overline{\mathrm{w}}(\overline{\mathrm{y}})_{\alpha_{\mathrm{j}}+\mathrm{k}}\right|=\sqrt{\frac{\operatorname{Crz}(\overline{\mathrm{y}})_{\mathrm{j}} \operatorname{ISn}_{\mathrm{j}} \overline{\mathrm{f}}_{\alpha_{\mathrm{j}}+\mathrm{k}}^{2}}{2}} \quad \text { para } \quad \mathrm{j}=0,1, \ldots,(\mathrm{n}-2) \\
& \left|\mathrm{w}(\overline{\mathrm{y}})_{\mathrm{N} / 2}\right|=\sqrt{\operatorname{Crz}(\overline{\mathrm{y}})_{\mathrm{n}-1} \operatorname{ISn}_{\mathrm{n}-1} \overline{\mathrm{f}}_{\mathrm{N} / 2}^{2}}
\end{aligned}
$$


Estes coeficientes das wavelets harmônicas, mostrados em (5.86) a (5.89), são a DHWT para uma ação em uma determinada altura ou largura de um modulo da estrutura. Em posse destes coeficientes, todas as etapas seguintes para a obtenção da ação são praticamente as mesmas, diferindo apenas na introdução do valor da (5.65) em (5.55):

$$
\begin{aligned}
& p\left(\overline{\mathrm{z}}, \mathrm{t}_{\mathrm{r}}\right)=p_{\mathrm{f}}(\overline{\mathrm{z}})\left(\sum_{\mathrm{k}=0}^{\mathrm{N}-1} \mathrm{X}(\overline{\mathrm{z}})_{\mathrm{k}} \mathrm{e}^{i(2 \pi \mathrm{kr} / \mathrm{N})}\right) \quad \text { para } \quad \mathrm{r}=0,1, \ldots,(\mathrm{N}-1) \\
& p\left(\mathrm{z}_{\mathrm{Gc}}, \overline{\mathrm{y}}, \mathrm{t}_{\mathrm{r}}\right)=p_{\mathrm{f}}\left(\mathrm{z}_{\mathrm{Gc}}\right)\left(\sum_{\mathrm{k}=0}^{\mathrm{N}-1} \mathrm{X}(\overline{\mathrm{y}})_{\mathrm{k}} \mathrm{e}^{i(2 \pi \mathrm{kr} / \mathrm{N})}\right) \quad \text { para } \quad \mathrm{r}=0,1, \ldots,(\mathrm{N}-1)
\end{aligned}
$$

Onde (5.90) e (5.91) são respectivamente, as pressões aplicadas para um módulo de uma estrutura alta e um modulo de uma estrutura larga, em posse das pressões calculadas para cada módulo, encontra-se os carregamento atuantes em m módulos através de:

$$
\begin{array}{lrr}
F\left(\overline{\mathrm{z}}_{\mathrm{k}}, \mathrm{t}\right)=\mathrm{Ca}_{\mathrm{k}}\left(p\left(\overline{\mathrm{z}}_{\mathrm{k}}, \mathrm{t}\right)+p_{\text {est }}\left(\overline{\mathrm{z}}_{\mathrm{k}}\right)\right) A_{\mathrm{k}} & \text { para } & \mathrm{k}=0,1, \ldots, \mathrm{m} \\
F\left(\mathrm{z}_{\mathrm{Gc}}, \overline{\mathrm{y}}_{\mathrm{k}}, \mathrm{t}\right)=\mathrm{Ca}_{\mathrm{k}}\left(p\left(\mathrm{z}_{\mathrm{Gc}}, \overline{\mathrm{y}}_{\mathrm{k}}, \mathrm{t}\right)+p_{\text {est }}\left(\mathrm{z}_{\mathrm{Gc}}\right)\right) A_{\mathrm{k}} & \text { para } & \mathrm{k}=0,1, \ldots, \mathrm{m}
\end{array}
$$

Sendo $\mathrm{Ca}_{\mathrm{k}}$ e $A_{\mathrm{k}}$ o coeficiente de arrasto e a área e do módulo k e $F$ a força atuante neste, a qual deve ser dividida e aplicada nos nós da estrutura dentro desta área. A aplicação destas forças na estrutura é feita pelo vetor $\{F\}$ sendo aplicado nos graus de liberdade da estrutura de direções coincidentes com a das pressões devido ao vento. Este vetor na equação do movimento da estrutura tem o posicionamento:

$[M]\{\ddot{u}(\mathrm{t})\}+[C]\{\dot{u}(\mathrm{t})\}+[K]\{u(\mathrm{t})\}=\{F\}$

Com as matrizes $[M],[C]$ e $[K]$ representando, respectivamente a matriz de massa, de amortecimento e de rigidez. $\mathrm{E}$ os vetores $\{u(\mathrm{t})\},\{\dot{u}(\mathrm{t})\},\{\ddot{u}(\mathrm{t})\}$ representando, respectivamente os vetores de deslocamentos, de velocidades e de acelerações dos graus de liberdade da estrutura. 
Seguindo o método proposto por Franco (1993), algumas condições devem ser observadas para a obtenção de melhores resultados na geração de ações desta natureza, como o número de harmônicos em pelo menos 11, sendo que, um dos harmônicos deva coincidir com a frequiência fundamental da estrutura, onde, para o intervalo de tempo entre 600 e 0,5 segundos, um valor em torno do oitavo harmônico é geralmente utilizado para um intervalo de freqüência em torno de $2 \mathrm{~Hz}$.

Franco (1993) ainda analisou que para 11 harmônicos, a contribuição do harmônico ressoante é superestimada por um fator da ordem de 2, pois o valor da integração de um intervalo do espectro de potência é concentrado em uma única freqüência, devido a isto, é recomendado reduzir pela metade o coeficiente ressonante e aumentar em $1 / 4$ os valores dos coeficientes adjacentes.

No entanto, com o aumento da quantidade de harmônicos este efeito é minimizado, podendo esta correção ser desprezada. Para o caso não estacionário, com o uso de DHWT, desenvolvido neste trabalho, o uso do espectro de potência dividido em 11 intervalos não é significativo, pois cada nível pode possuir vários coeficientes, distribuindo assim os valores do intervalo da integral do espectro de potência em várias freqüências. 


\subsection{UTILIZAÇÃO DAS AÇÕES PARA SISMO}

A intensidade dos sismos é fornecida pelas Normas de cada país com zonas sísmicas determinadas sobre o mapa deste país, juntamente com a composição do solo, é obtido um espectro de resposta da estrutura analisada. Esta forma de análise é bastante questionável, e segundo Corbani (2006), é dificil definir um melhor critério para este fator, visto que as forças ao longo do tempo geradas por ações sísmicas são forças inerciais, dadas matematicamente pelo produto das massas da estrutura excitada pelo histórico de um sinal de aceleração do solo (acelerograma).

Assim, as forças envolvidas em sismos dependem, da intensidade das acelerações verticais e horizontais do solo, da quantidade de massas e sua distribuição na estrutura, assim como a dissipação de energia do material que compões a estrutura e o comportamento reológico deste.

Além da questão da intensidade da aceleração, outro problema ocorre em relação a sua variação no tempo, embora em alguns trabalhos esta variação seja representada por uma simulação aleatória de comportamento estacionário, a não estacionaridade do sinal começa a ser considerada como um fator importante para uma representação mais realista da resposta da estrutura.

A semelhança existente entre a metodologia para simular carregamentos devido ao vento e devido a sismos favorece a migração de um caso para outro. No entanto, algumas adaptações devem ser feitas. As densidades espectrais de potência para sismos, geralmente, são construídas pela relação entre os valores das freqüências angulares e das acelerações do solo ao quadrado por freqüência angular.

Segundo Corbani (2006), estes espectros tendem a ter muitos picos e requerem ajustes para fins de projeto. Eles são, em geral, resultantes de médias de vários terremotos normalizados para a mesma aceleração de pico e sofrem um processo de ajuste de curvas. A idealização mais simples de um espectro de potência de sismos é o ruído branco ideal:

$\mathrm{W}_{a c}(\omega)=\mathrm{S}_{0} \quad$ para $\quad 0<\omega<\infty$ 
No entanto, este espectro é pouco realista, pois representa uma potência infinita. Uma forma mais realista é o modelo de ruído branco filtrado da formulação que se segue, conhecido como modelo de Kanai-Tajimi (1960).

$$
\mathrm{W}_{a c}(\omega)=\mathrm{S}_{0} \frac{\mathrm{r}_{\omega}\left(1+4 \xi_{\mathrm{g}}{ }^{2} \mathrm{r}_{\omega}{ }^{2}\right)}{\left(\left(1-\mathrm{r}_{\omega}{ }^{2}\right)^{2}+4 \xi_{\mathrm{g}}{ }^{2} \mathrm{r}_{\omega}{ }^{2}\right)} \quad \text { para } \quad \mathrm{r}_{\omega}=\left(\frac{\omega}{\omega_{\mathrm{g}}}\right)
$$

Nesse modelo, o movimento do solo é baseado na resposta em aceleração absoluta de um sistema massa-amortecedor-mola de um grau de liberdade sujeito a uma aceleração de base de um espectro tipo ruído branco ideal. Onde $\mathrm{S}_{0}, \xi_{\mathrm{g}}$ e $\omega_{\mathrm{g}}$ são respectivamente o espectro tipo ruído branco ideal, a taxa de amortecimento e a freqüência angular fundamental do solo.

Entretanto, a dificuldade de uso deste espectro reside na estimativa da intensidade de $\mathrm{S}_{0}$, pois este parâmetro precisa de registros locais de terremotos anteriores e a análise do perfil geológico do solo. Gouold e Abu-Sitta (1980) minimizam esta dificuldade com algumas variações em (5.96):

$$
\frac{\mathrm{W}_{a c}(\omega)}{\sigma_{a c}{ }^{2}}=\frac{4 \xi_{\mathrm{g}}}{\omega_{\mathrm{f}} \pi\left(1+4 \xi_{\mathrm{g}}{ }^{2}\right)} \frac{\mathrm{r}_{\omega}\left(1+4 \xi_{\mathrm{g}}{ }^{2} \mathrm{r}_{\omega}{ }^{2}\right)}{\left(\left(1-\mathrm{r}_{\omega}{ }^{2}\right)^{2}+4 \xi_{\mathrm{g}}{ }^{2} \mathrm{r}_{\omega}{ }^{2}\right)} \quad \text { para } \quad \mathrm{r}_{\omega}=\left(\frac{\omega}{\omega_{\mathrm{g}}}\right)
$$

Onde $\sigma_{a c}{ }^{2}$ é o desvio padrão da aceleração do solo, pois o espectro $\mathrm{W}_{a c}(\omega)$ dividido pelo desvio padrão gera um valor de dimensão pertencente ao inverso da freqüência angular $(1 / \omega)$. De acordo com Gouold e Abu-Sitta (1980), a taxa de amortecimento do solo em geral, varia entre 0,6 e 0,7 e a freqüência angular fundamental do solo, entre $4 \pi$ e $6 \pi$. Corbani (2006), com base na formulação de (5.97), utiliza o seguinte espectro reduzido:

$$
\mathrm{W}_{a c}(\omega)=R^{2} \frac{4 \xi_{\mathrm{g}}}{\omega \pi\left(1+4 \xi_{\mathrm{g}}{ }^{2}\right)} \frac{\mathrm{r}_{\omega}\left(1+4 \xi_{\mathrm{g}}{ }^{2} \mathrm{r}_{\omega}{ }^{2}\right)}{\left(\left(1-\mathrm{r}_{\omega}{ }^{2}\right)^{2}+4 \xi_{\mathrm{g}}{ }^{2} \mathrm{r}_{\omega}{ }^{2}\right)} \quad \text { para } \quad \mathrm{r}_{\omega}=\left(\frac{\omega}{\omega_{\mathrm{g}}}\right)
$$

Onde:

$$
R=\frac{\ddot{u}_{\max }^{\mathrm{N}}}{\ddot{u}_{\max }^{\mathrm{s}}}
$$


Sendo $R$ uma constante, com dimensões de aceleração, onde $\ddot{u}_{\max }^{\mathrm{N}}$ é aceleração máxima do espectro de resposta da estrutura de alguma norma, e $\ddot{u}_{\max }^{\mathrm{s}}$ representa a aceleração máxima obtida através de um sistema massa-amortecedor-mola para um grau de liberdade, com comportamento linearmente elástico, sob a ação de acelerações obtidas de forma aleatória e vindas de um espectro de potência adimensional como em (5.97).

O acelerograma pode ser representado, de acordo com Gould e Abu-Sitta (1980), aproximadamente, por um número finito de funções harmônicas convenientemente escolhidas com períodos entre 1 a 0,2 segundos, equivalente as freqüências de 1 a $5 \mathrm{~Hz}$, de modo a abranger pelo menos o primeiro modo de vibração da estrutura. Adota-se então uma quantidade de $\mathrm{n}$ harmônicos, os quais um contenha a freqüência angular $\omega_{\mathrm{f}}$, coincidente com o primeiro modo de vibração da estrutura. Devido a isto, a partir de (5.1), tem-se:

$\mathrm{C}^{\mathrm{F}}{ }_{\mathrm{i}}=\sqrt{2 \int_{\omega^{\mathrm{F}-}}^{\omega_{\mathrm{i}}^{\mathrm{F}+}} \frac{\mathrm{W}_{a c}(\omega)}{\sigma_{a c}{ }^{2}} \mathrm{~d} \omega} \quad$ para $\quad \mathrm{i}=1,2, \ldots, \mathrm{n}$

Percebe-se, neste caso, que o coeficiente do harmônico é adimensional, pois o domínio de integração é a freqüência angular. Os intervalos de integração ocorrem de acordo com as relações (5.2) e (5.3). Quando se necessita gerar um comportamento não estacionário, a variável tempo é necessária, esta é introduzida através da função de tendência, que a partir da equação (5.28), tem-se:

$\mathrm{W}(\omega, \mathrm{t})=\frac{\mathrm{W}_{a c}(\omega)}{\sigma_{a c}{ }^{2}} \mathrm{f}(\mathrm{t})^{2}$

Então baseado em (5.32) e (5.33), chega-se:

$$
\begin{aligned}
& \mathrm{IS}_{\mathrm{j}}=\int_{\omega^{\mathrm{w}-}{ }_{\mathrm{j}}}^{\omega_{\mathrm{j}}^{\omega+}} \frac{\mathrm{W}_{a c}(\omega)}{\sigma_{a c}{ }^{2}} \mathrm{~d} \omega \quad \text { para } \mathrm{j}=0,1, \ldots,(\mathrm{n}-2) \\
& \mathrm{IS}_{\mathrm{n}-1}=\int_{\omega^{\mathrm{w}}{ }_{\mathrm{n}-1}-1}^{\omega^{\mathrm{w}+}} \frac{\mathrm{W}_{a c}(\omega)}{\sigma_{a c}{ }^{2}} \mathrm{~d} \omega
\end{aligned}
$$


Onde os intervalos de integração ocorrem de acordo com as relações (5.35) a (5.38). Geralmente na análise de sismos, são aplicadas acelerações horizontais devido ao fato das verticais terem, geralmente, uma menor magnitude. No entanto, dependendo da estrutura e da finalidade da análise, ambas as direções podem ser aplicadas na matriz de massa da estrutura, resultando, em maior variedade de forças inerciais.

A aplicação das ações de sismos na estrutura é feita pelo vetor $\left\{\ddot{u}_{g}(t)\right\}$ que representa a aceleração do sismo no solo, sendo aplicada nos graus de liberdade da estrutura de direções coincidentes com a aceleração do solo. Este vetor, na equação do movimento da estrutura, gera esforços inerciais, devido a isto, tem o posicionamento inicial:

$[M]\left\{\{\ddot{u}(\mathrm{t})\}+\left\{\ddot{u}_{g}(\mathrm{t})\right\}\right\}+[C]\{\dot{u}(\mathrm{t})\}+[K]\{u(\mathrm{t})\}=\{0\}$

Com as matrizes $[M],[C]$ e $[K]$ representando, respectivamente a matriz de massa, de amortecimento e de rigidez. $\mathrm{E}$ os vetores $\{u(\mathrm{t})\},\{\dot{u}(\mathrm{t})\},\{\ddot{u}(\mathrm{t})\}$ representando, respectivamente os vetores de deslocamentos, de velocidades e de acelerações dos graus de liberdade da estrutura, por ultimo, o vetor $\{0\}$ que representa o vetor nulo, pois considera-se neste caso que a única ação sobre a estrutura é o sismo. Para a resolução da equação do movimento, a parcela da aceleração devido ao solo se desloca para a direita: $[M]\{\ddot{u}(\mathrm{t})\}+[C]\{\dot{u}(\mathrm{t})\}+[K]\{u(\mathrm{t})\}=-[M]\left\{\ddot{u}_{g}(\mathrm{t})\right\}$

Para uma boa simulação do acelerograma de um terremoto, é recomendável utilizar pelo menos o número mínimo de funções harmônicas do processo do "Vento Sintético", sugerida por Franco (1993), entretanto, segundo Corbani (2006), a função harmônica com a frequiência ressonante com valor em torno do sétimo harmônico é geralmente utilizado quando houver um número de 11 harmônicos para um intervalo de freqüência em torno de $5 \mathrm{~Hz}$.

Foi observado neste trabalho que para 11 harmônicos, a contribuição do harmônico ressoante é também superestimada, pois o valor da integração de um intervalo do espectro de potência é concentrado em uma única freqüência, sendo a correção sugerida por Franco (1993) também útil para melhores resultados. 
No entanto, igualmente ao caso do vento, com o aumento da quantidade de harmônicos este efeito é minimizado, podendo esta correção ser desprezada. Para o caso não estacionário, com o uso de DHWT, desenvolvido neste trabalho, o uso do espectro de potência dividido em 11 intervalos não é significativo, pois cada nível pode possuir vários coeficientes, distribuindo assim os valores do intervalo da integral do espectro de potência em várias frequiências. 


\section{EXEMPLOS NUMÉRICOS}

\subsection{INTRODUÇÃO}

Neste capítulo, o modelo de geração de ação desenvolvido neste trabalho é aplicado em modelos estruturais para um melhor entendimento de suas vantagens e desvantagens em relação ao modelo mais conhecido, que é baseado no modelo do vento sintético o qual utiliza sua geração através de Série de Fourier.

Mas antes é necessário escolher o modelo estrutural, optou-se por utilizar o shear building pela facilidade do desenvolvimento de seu algoritmo, também foi adotado o modelo elasto-plástico perfeito com formação de rótula plástica para o comportamento do material empregado, pois com um comportamento não-linear as diferenças dos modelos de geração de ações com comportamento estacionário e não estacionário podem ficar mais evidentes.

Nas próximas seções, são abordados de formas sucintas o modelo estrutural, do material e do método de integração implícita adotado, que para este trabalho é o método beta de Newmark, como descrito por Paz e Leigh (2004). Por ultimo são abordados os exemplos numéricos, onde todas as ações geradas assim como as respostas estruturais foram obtidas devido a algoritmos desenvolvidos para este trabalho na plataforma MATLAB. 


\subsection{MODELO DE SHEAR BUILDING}

Para os exemplos mostrados neste trabalho é utilizada a análise dinâmica por método implícito de integração direta passo-a-passo no tempo do modelo de estrutura shear building, para a análise de edifícios com pilares metálicos de múltiplos andares. Na Figura 6.1, observa-se a numeração adotada e as características da estrutura para a formulação deste problema.

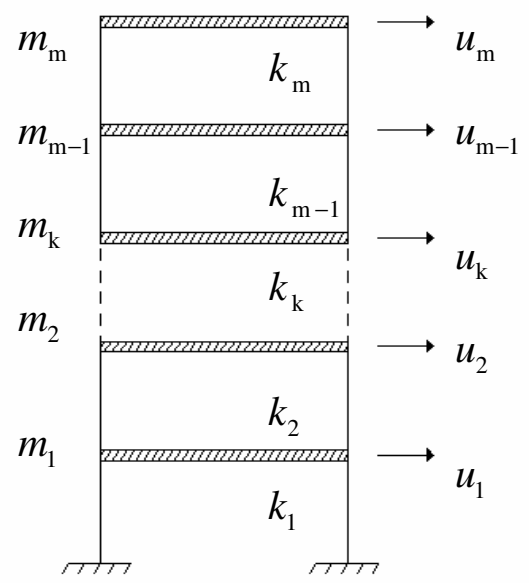

Figura 6.1. Numeração adotada para o modelo de shear building.

Para a matriz de massa é adotado um modelo de lumped mass para definir a matriz de massa, conforme apresentado em Clough (1975). Para este modelo admite-se que a massa total se concentra nos pontos em que os deslocamentos transversais são definidos. Desta forma a matriz $[M]$ será diagonal, na forma:

$$
[M]=\left[\begin{array}{cccccc}
m_{1} & 0 & \cdots & 0 & \cdots & 0 \\
0 & m_{2} & \cdots & 0 & \cdots & 0 \\
\vdots & \vdots & \ddots & \vdots & \ddots & \vdots \\
0 & 0 & \cdots & m_{\mathrm{k}} & \cdots & 0 \\
\vdots & \vdots & \ddots & \vdots & \ddots & \vdots \\
0 & 0 & \cdots & 0 & \cdots & m_{\mathrm{m}}
\end{array}\right]
$$

Para o modelo de rigidez adotado nas colunas é também descrito em Clough (1975) em que se admite uma rigidez alta nas lajes, assim o apoio dos pilres nas lajes é equivalente a um engastamento. No comportamento elástico linear, pode-se dizer que a rigidez total que representa os 4 pilares entre duas lajes, é dada pela equação: 
$k_{\mathrm{k}}=4\left(\frac{12 \mathrm{EI}_{\mathrm{k}}}{\ell_{\mathrm{k}}^{3}}\right)=\frac{48 \mathrm{EI}_{\mathrm{k}}}{\ell_{\mathrm{k}}^{3}}$

Onde E representa o módulo de elasticidade do material, que para o caso do aço, neste trabalho, o valor adotado é $210000 \mathrm{MPa}$. $\mathrm{I}_{\mathrm{k}}$ é o momento de inércia de um perfil metálico das colunas para cada pavimento e, por ultimo, $\ell_{\mathrm{k}}$ indica o comprimento do pilar, determinado pela distância entre a laje inferior e a laje superior também para cada pavimento. Então a matriz de rigidez global tem a seguinte forma:

$$
[K]=\left[\begin{array}{cccccccc}
k_{1}+k_{2} & -k_{2} & \cdots & 0 & 0 & \cdots & 0 & 0 \\
-k_{2} & k_{2}+k_{3} & \cdots & 0 & 0 & \cdots & 0 & 0 \\
\vdots & \vdots & \ddots & \vdots & \vdots & \ddots & \vdots & \vdots \\
0 & 0 & \cdots & k_{\mathrm{k}-1}+k_{\mathrm{k}} & -k_{\mathrm{k}} & \cdots & 0 & 0 \\
0 & 0 & \cdots & -k_{\mathrm{k}} & k_{\mathrm{k}}+k_{\mathrm{k}+1} & \cdots & 0 & 0 \\
\vdots & \vdots & \ddots & \vdots & \vdots & \ddots & \vdots & \vdots \\
0 & 0 & \cdots & 0 & 0 & \cdots & k_{\mathrm{m}-1}+k_{\mathrm{m}} & -k_{\mathrm{m}} \\
0 & 0 & \cdots & 0 & 0 & \cdots & -k_{\mathrm{m}} & k_{\mathrm{m}}
\end{array}\right]
$$

Neste trabalho também é considerado análise com comportamentos elástico linear e elasto-plástico perfeito do aço, este ultimo comportamento mencionado pode ser obsevado:

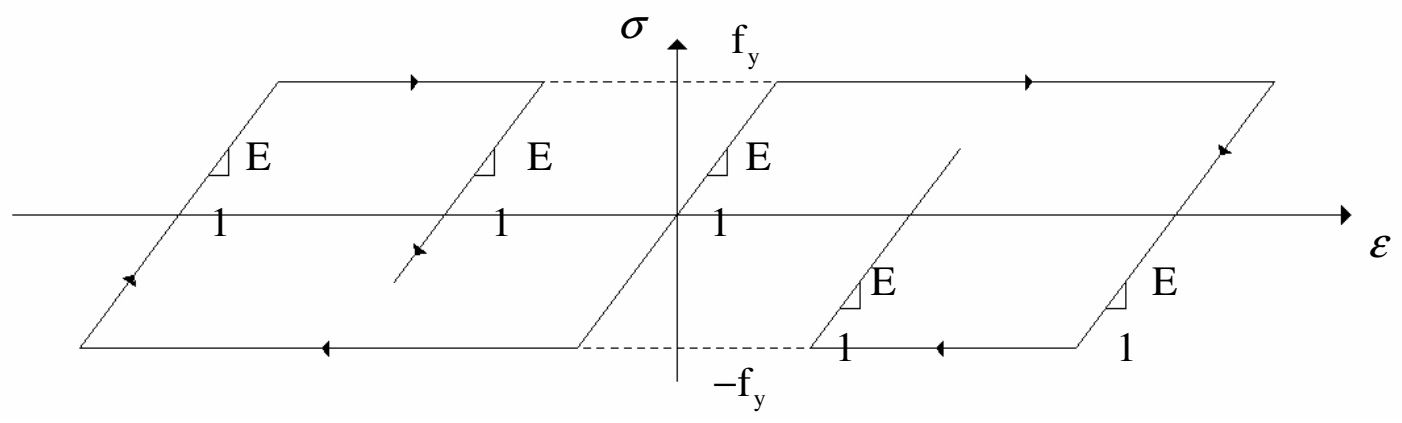

Figura 6.2. Modelo de material elasto-plástico perfeito.

No gráfico tensãoxdeformação ( $\sigma \times \varepsilon$ ) observado na figura 6.2, após atingir a tensão de escoamento $\mathrm{f}_{\mathrm{y}}$ do material, a ocorrência de um decréscimo de solicitações faz retornar ao comportamento elástico, permanecendo, no entanto, uma deformação residual. Neste trabalho, o valor adotado é da tensão de escoamento é de $250 \mathrm{MPa}$. 
Como o modelo de shear building utiliza elementos de barra, possuindo assim graus de liberdade de rotação e esforço de flexão, é aplicado o modelo de rótula plástica, que consiste na anulação da rigidez a rotação em determinado nó, com ele pode-se adicionar rotação sem alteração do momento fletor, pois se considera que a seção não consiga mais absorver energia. $\mathrm{O}$ momento fletor extremo aplicado à rótula é o momento de plastificação total, este para a seção de um pilar em um pavimento k, é obtido por:

$\mathrm{Mpl}_{\mathrm{k}}=\mathrm{f}_{\mathrm{y}} Z_{\mathrm{k}}$

Onde $Z_{\mathrm{k}}$ representa o módulo de resistência plástica. Neste trabalho é utilizado o esforço cortante como um dos critérios de surgimento de rótula plástica, este, possui o valor máximo que pode ser suportado pelos pilares de um pavimento:

$$
\mathrm{Qpl}_{\mathrm{k}}=4\left(\frac{2 \mathrm{Mpl}_{\mathrm{k}}}{\ell_{\mathrm{k}}}\right)=\frac{8 \mathrm{Mpl}_{\mathrm{k}}}{\ell_{\mathrm{k}}} \quad \text { para } \quad \mathrm{k}=1,2, \ldots, \mathrm{m}
$$

Sendo o número 4 a quantidade de pilares em um pavimento, e o número 2 ocorrendo pela composição do momento da extremidade superior e inferior da cada pilar. O outro critério de surgimento de rótula plástica é o sentido da rotação dos pilares de um pavimento, como para as condições de contorno do modelo elástico linear todas as rotações possuem valores nulos para o shear building, a rotação dos pilares de um pavimento $\mathrm{k}$ em um passo r é calculada por:

$$
\begin{array}{lll}
\Delta \rho_{\mathrm{k}, \mathrm{r}}=\frac{u_{\mathrm{k}, \mathrm{r}}}{\ell_{\mathrm{k}}} & \text { para } & \mathrm{k}=1 \\
\Delta \rho_{\mathrm{k}, \mathrm{r}}=\frac{u_{\mathrm{k}, \mathrm{r}}-u_{\mathrm{k}-1, \mathrm{r}}}{\ell_{\mathrm{k}}} & \text { para } & \mathrm{k}=2, \ldots, \mathrm{m}
\end{array}
$$

Em posse desses dois critérios, este modelo, baseado no modelo de Paz e Leigh (2004), impõe as seguintes condições para a formação de rótula plástica nos pilares de um pavimento k:

$$
\begin{array}{lllll}
k_{\mathrm{k}, \mathrm{r}}=0 & \text { se } & \mathrm{Q}_{\mathrm{k}, \mathrm{r}}=\mathrm{Qpl}_{\mathrm{k}} \text { e } \Delta \rho_{\mathrm{k}, \mathrm{r}}>0, & \text { para } & \mathrm{r}=1,2, \ldots, \mathrm{N}+1 \\
k_{\mathrm{k}, \mathrm{r}}=0 & \text { se } & \mathrm{Q}_{\mathrm{k}, \mathrm{r}}=-\mathrm{Qpl}_{\mathrm{k}} \text { e } \Delta \rho_{\mathrm{k}, \mathrm{r}}<0, & \text { para } & \mathrm{r}=1,2, \ldots, \mathrm{N}+1 \\
k_{\mathrm{k}, \mathrm{r}}=\frac{48 \mathrm{EI}_{\mathrm{k}}}{\ell_{\mathrm{k}}{ }^{3}} & \text { se } & -\mathrm{Qpl}_{\mathrm{k}}<\mathrm{Q}_{\mathrm{k}, \mathrm{r}}<\mathrm{Qpl}_{\mathrm{k}}, & \text { para } & \mathrm{r}=1,2, \ldots, \mathrm{N}+1
\end{array}
$$


$\begin{array}{llllll}k_{\mathrm{k}, \mathrm{r}}=\frac{48 \mathrm{EI}_{\mathrm{k}}}{\ell_{\mathrm{k}}{ }^{3}} & \text { se } & \mathrm{Q}_{\mathrm{k}, \mathrm{r}}=\mathrm{Qpl}_{\mathrm{k}} \quad \text { e } \Delta \rho_{\mathrm{k}, \mathrm{r}}<0, & \text { para } & \mathrm{r}=1,2, \ldots, \mathrm{N}+1 \\ k_{\mathrm{k}, \mathrm{r}}=\frac{48 \mathrm{EI}_{\mathrm{k}}}{\ell_{\mathrm{k}}{ }^{3}} & \text { se } & \mathrm{Q}_{\mathrm{k}, \mathrm{r}}=-\mathrm{Qpl}_{\mathrm{k}} \text { e } \Delta \rho_{\mathrm{k}, \mathrm{r}}>0, \quad \text { para } & \mathrm{r}=1,2, \ldots, \mathrm{N}+1\end{array}$

Onde (6.8) e (6.9) impõem que para cada passo $r$, a formação da rótula plástica $\left(k_{\mathrm{k}, \mathrm{r}}=0\right)$ ocorre quando o cortante atinge o valor de plastificação e a rotação de seu pilar possui o mesmo sentido do cortante de plastificação; a condição (6.10) impõe que para valores de cortantes intermediários não há formação de rótula plástica, independente do sentido da rotação de seu pilar; e as condições (6.11) e (6.12) impõem que havendo um desacordo entre os sentidos do cortante de plastificação e da rotação de seu pilar, a rótula plástica é removida, sendo então a rigidez restaurada.

Para a determinação da matriz de amortecimento, é adotado o método proposto por Rayleigh e apresentado por Tedesco, McDouglas e Ross (1999), com a modificação aplicada neste trabalho de alterá-la a cada passo $r$, assim como matriz de rigidez no comportamento plástico.

A estimativa das taxas de amortecimento relativas a certo número de modos escolhidos, em geral as duas menores frequiências de vibração da estrutura é necessária. Estimadas estas taxas de amortecimento, a matriz de amortecimento de Rayleigh modificada a cada passo $r$ pode ser escrita como:

$[C]_{\mathrm{r}}=r_{1}[M]+r_{2}[K]_{\mathrm{r}}$

Onde $r_{1}$ e $r_{2}$ são obtidos através da resolução do sistema:

$\frac{1}{2}\left[\begin{array}{cc}\frac{1}{\omega_{1}} & \omega_{1} \\ \frac{1}{\omega_{2}} & \omega_{2}\end{array}\right]\left[\begin{array}{l}r_{1} \\ r_{2}\end{array}\right]=\left[\begin{array}{l}\xi_{1} \\ \xi_{2}\end{array}\right]$

Sendo $\omega_{1}$ e $\omega_{2}$ as duas primeiras freqüências naturais angulares de vibração da estrutura, com $\xi_{1}$ e $\xi_{2}$ as respectivas taxas de amortecimento estimadas para a estrutura. 
Neste trabalho é utilizado como método de integração direta passo-à-passo no tempo o método Beta de Newmark, que segundo Paz e Leigh (2004), pode ser considerado como uma generalização do método de Newmark com aceleração linear, pois faz as seguintes considerações:

$$
\begin{aligned}
& \{\Delta \dot{u}\}_{\mathrm{r}}=\{\ddot{u}\}_{\mathrm{r}} \Delta t+\frac{1}{2}\{\Delta \ddot{u}\}_{\mathrm{r}} \Delta t \Rightarrow\{\Delta \dot{u}\}_{\mathrm{r}}=\{\ddot{u}\}_{\mathrm{r}} \Delta t+\gamma\{\Delta \ddot{u}\}_{\mathrm{r}} \Delta t \\
& \{\Delta u\}_{\mathrm{r}}=\{\dot{u}\}_{\mathrm{r}} \Delta t+\frac{1}{2}\{\ddot{u}\}_{\mathrm{r}} \Delta t^{2}+\frac{1}{6}\{\Delta \ddot{u}\}_{\mathrm{r}} \Delta t^{2} \Rightarrow\{\Delta u\}_{\mathrm{r}}=\{\dot{u}\}_{\mathrm{r}} \Delta t+\frac{1}{2}\{\ddot{u}\}_{\mathrm{r}} \Delta t^{2}+\beta\{\Delta \ddot{u}\}_{\mathrm{r}} \Delta t^{2}
\end{aligned}
$$

No entanto, Paz e Leigh (2004) recomendam que $\gamma=1 / 2$ para evitar amortecimentos desnecessários no sistema, e que $\beta$ tenha uma variação entre $1 / 6 \leq \beta \leq 1 / 2$, onde para 1/6 é exatamente igual ao método com aceleração linear, e para 1/4 é igual ao método de Newmark com aceleração constante. Para a utilização do método são necessárias as matrizes $[K]_{\mathrm{r}},[M]$ e $[C]_{\mathrm{r}}$ juntamente com os vetores $\{u\}_{\mathrm{r}},\{\dot{u}\}_{\mathrm{r}}$ e $\{F\}_{\mathrm{r}}$ para $\mathrm{r}=1$.

Então, calcula-se a aceleração inicial:

$$
\{\Delta \ddot{u}(\mathrm{t})\}_{\mathrm{r}}=[M]^{-1}\left(\{\Delta F\}_{\mathrm{r}}-[C]_{\mathrm{r}}\{\Delta \dot{u}(\mathrm{t})\}_{\mathrm{r}}-[K]_{\mathrm{r}}\{\Delta u(\mathrm{t})\}_{\mathrm{r}}\right) \text { para } \quad \mathrm{r}=1
$$

São escolhidos os valores de $\beta$ e $\Delta t$, e calculados os incrementos do carregamento:

$$
\{\Delta F\}_{\mathrm{r}}=\{F\}_{\mathrm{r}+1}-\{F\}_{\mathrm{r}} \quad \text { para } \quad \mathrm{r}=1,2, \ldots, \mathrm{N}
$$

Com estes dados, para cada passo r é calculada o incremento de carregamento efetivo:

$$
\{\Delta \bar{F}\}_{\mathrm{r}}=\{\Delta F\}_{\mathrm{r}}+\left(\frac{1}{\beta \Delta t}[M]+\frac{1}{2 \beta}[C]_{\mathrm{r}}\right)\{\dot{u}\}_{\mathrm{r}}+\left(\frac{1}{2 \beta}[M]-\left(1-\frac{1}{4 \beta}\right) \Delta t[C]_{\mathrm{r}}\right)\{\ddot{u}\}_{\mathrm{r}}
$$

E a matriz de rigidez efetiva:

$$
[\bar{K}]_{\mathrm{r}}=[K]_{\mathrm{r}}+\frac{1}{\beta \Delta t^{2}}[M]+\frac{1}{2 \beta \Delta t}[C]_{\mathrm{r}}
$$

Em posse de (6.19) e (6.20), é encontrado o vetor do incremento de deslocamento através de:

$$
[\bar{K}]_{\mathrm{r}}\{\Delta u\}_{\mathrm{r}}=\{\Delta \bar{F}\}_{\mathrm{r}} \Rightarrow\{\Delta u\}_{\mathrm{r}}=[\bar{K}]_{\mathrm{r}}^{-1}\{\Delta \bar{F}\}_{\mathrm{r}}
$$


Com (6.21) é possível achar os vetores do incremento de velocidade e aceleração, através de relações desenvolvidas a partir (6.15) e (6.16):

$$
\begin{aligned}
& \{\Delta \dot{u}\}_{\mathrm{r}}=\frac{1}{2 \beta \Delta t}\{\Delta u\}_{\mathrm{r}}-\frac{1}{2 \beta}\{\dot{u}\}_{\mathrm{r}}+\left(1-\frac{1}{4 \beta}\right) \Delta t\{\ddot{u}\}_{\mathrm{r}} \\
& \{\Delta \ddot{u}\}_{\mathrm{r}}=\frac{1}{\beta \Delta t^{2}}\{\Delta u\}_{\mathrm{r}}-\frac{1}{\beta \Delta t}\{\dot{u}\}_{\mathrm{r}}-\frac{1}{2 \beta}\{\ddot{u}\}_{\mathrm{r}}
\end{aligned}
$$

E finalmente, são gerados os vetores de deslocamento, velocidade e aceleração para o passo seguinte:

$$
\begin{aligned}
& \{u\}_{\mathrm{r}+1}=\{u\}_{\mathrm{r}}+\{\Delta u\}_{\mathrm{r}} \\
& \{\dot{u}\}_{\mathrm{r}+1}=\{\dot{u}\}_{\mathrm{r}}+\{\Delta \dot{u}\}_{\mathrm{r}} \\
& \{\ddot{u}\}_{\mathrm{r}+1}=\{\ddot{u}\}_{\mathrm{r}}+\{\Delta \ddot{u}\}_{\mathrm{r}}
\end{aligned}
$$

Este processo é repetido ao todo $\mathrm{N}$ vezes, sendo a condição de equalização das forças garantida pela equação do movimento para cada passo r:

$$
[M]\{\Delta \ddot{u}(\mathrm{t})\}_{\mathrm{r}}+[C]\{\Delta \dot{u}(\mathrm{t})\}_{\mathrm{r}}+[K]_{\mathrm{r}}\{\Delta u(\mathrm{t})\}_{\mathrm{r}}=\{\Delta F\}_{\mathrm{r}}
$$

Pois através do uso de (6.22) e (6.23) em (6.27), chega-se a (6.21). Quando o shear bulding é analisado com o comportamento elasto-plástico perfeito, a cada incremento de tempo é verificado o cortante dos pilares de cada pavimento:

$$
\begin{array}{lll}
\Delta \mathrm{Q}_{\mathrm{k}, \mathrm{r}}=k_{\mathrm{k}} \Delta u_{\mathrm{k}, \mathrm{r}} & \text { para } & \mathrm{k}=1 \\
\Delta \mathrm{Q}_{\mathrm{k}, \mathrm{r}}=k_{\mathrm{k}}\left(\Delta u_{\mathrm{k}, \mathrm{r}}-\Delta u_{\mathrm{k}-1, \mathrm{r}}\right) & \text { para } & \mathrm{k}=2,3, \ldots, \mathrm{m} \\
\mathrm{Q}_{\mathrm{k}, \mathrm{r}+\mathrm{r}}=\mathrm{Q}_{\mathrm{k}, \mathrm{r}}+\Delta \mathrm{Q}_{\mathrm{k}, \mathrm{r}} & \text { para } & \mathrm{k}=1,2, \ldots, \mathrm{m}
\end{array}
$$

Aonde (6.28) e (6.29) vem do produto da matriz de rigidez local do elemento de barra com o vetor do incremento de deslocamento, e devido à (6.6), (6.7) e (6.30), para cada passo r são analisada as condições (6.8) a (6.12), resultando na montagem da matriz $[K]_{\mathrm{r}}$, e consequentemente, da matriz $[C]_{\mathrm{r}}$. 


\subsection{EXEMPLO 1}

Este primeiro exemplo é na verdade um prólogo para os outros exemplos, pois este não analisa a ação na estrutura e sim as características dos modelos de geração de ações. A partir deste conceito é definida a seguinte função em relação ao tempo:

$f(t)=\cos (7.2 t)^{3}+\cos (15.7 t)^{5}$

Na Figura 6.3 pode ser obtida a visualização de (6.31):

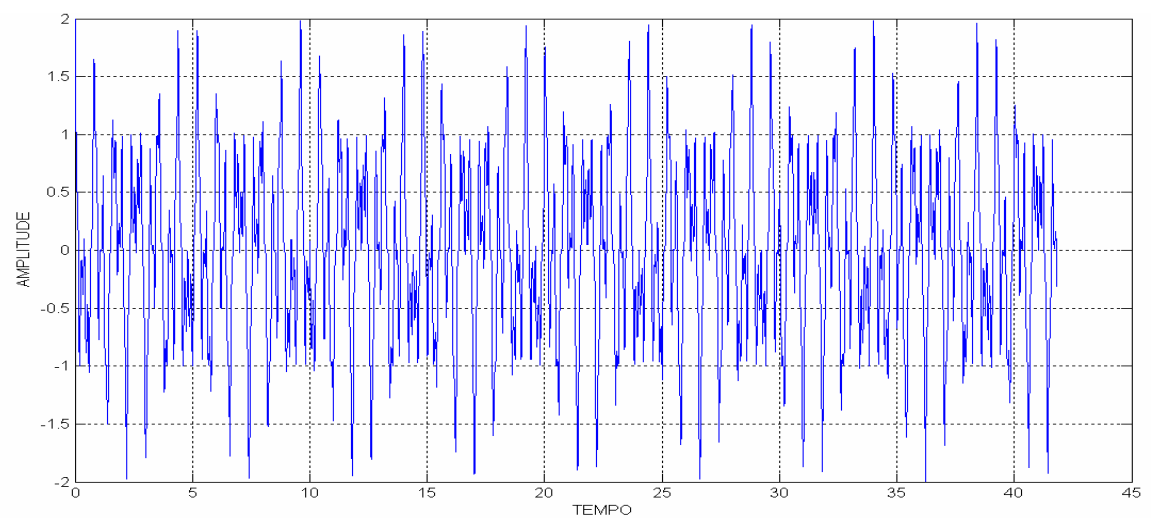

Figura 6.3. Função em relação ao tempo.

Com a obtenção de 2092 pontos com espaçamento de 0.02 entre eles, é então obtido o espectro de potência desta função temporal através do uso de DFT:

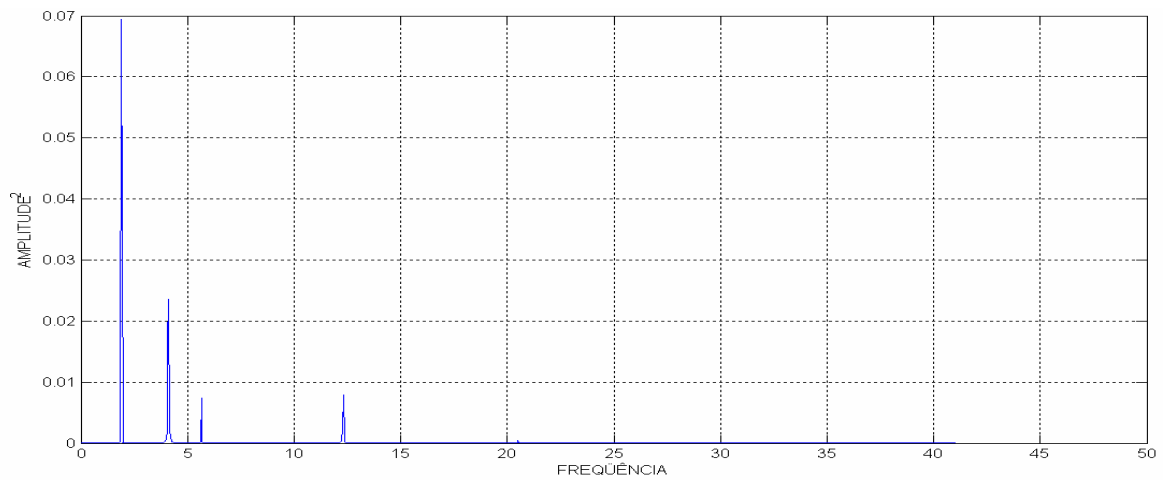

Figura 6.4. Espectro de potência da função temporal.

Em posse destes mesmos dados, é possível também obter o escalograma desta função temporal através do uso de DHWT: 


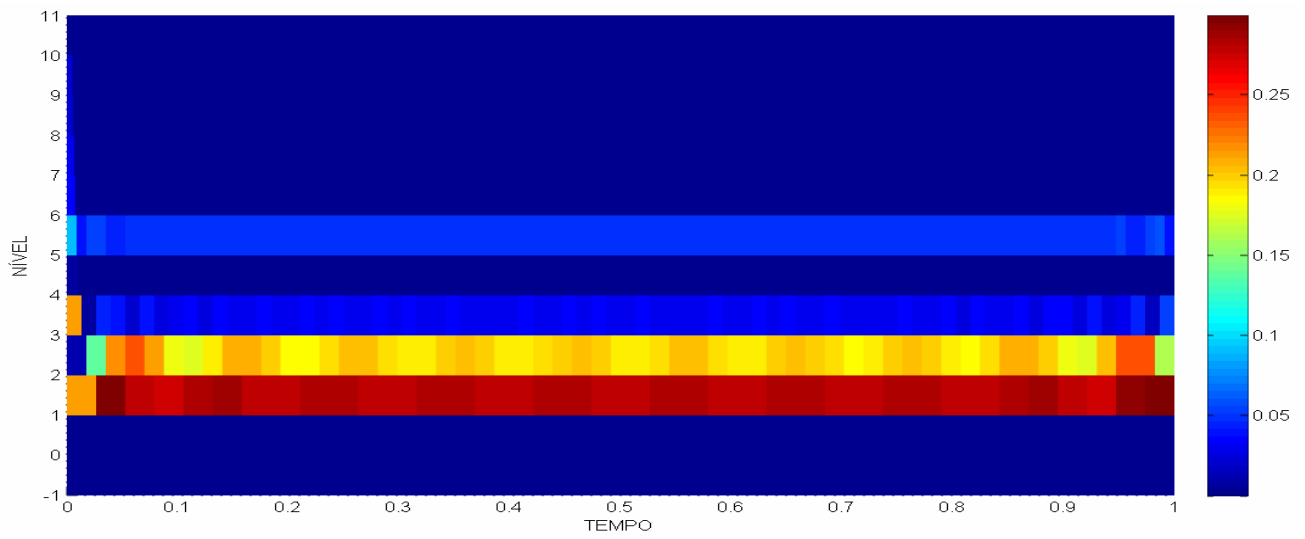

Figura 6.5. Escalograma da função temporal, com p=6 e n=11.

Estas três figuras possuem três domínios diferentes, que são respectivamente o tempo, a frequiência, e ambos no caso do escalograma, no entanto, todos estes possuem o mesmo tipo de imagem que é relacionada à amplitude.

Neste exemplo, em particular, foi dado à amplitude perfeitamente definida no tempo, através da função temporal descrita pela função (6.31), de maneira análoga, para a geração de uma ação de comportamento aproximadamente estacionário como os modelos do vento e do sismo sintético, são usados espectros como os de Davenport e Kanai-Tajimi que estabelecem a potência, sendo esta oriunda da amplitude, definida então, em função da freqüência, resultando em um descontrole tão grande no domínio do tempo que se torna praticamente impossível definir uma tendência em função deste último.

No entanto, quando se utiliza um escalograma, tanto o tempo e a freqüência possuem alguma definição, embora estas não sejam tão exatas, sendo explicado pelo principio da incerteza, estas definições já podem explicitar tendências de ambos os domínios.

Devido a estes fatores, para este método de geração de ação com comportamento não estacionário, são utilizados os espectros como os de Davenport e Kanai-Tajimi, que fornecem informação em função da frequiência, e funções de tendência, que fornecem informação em função do tempo, onde a composição destes fornece um escalograma para a obetenção de determinadas tendências nos dois domínios, sendo a maior influência de um domínio ou de outro, estabelecida pela relação da taxa de variação da janela tempo-frequência, onde algumas foram definidas neste trabalho, no capítulo 4. 


\subsection{EXEMPLO 2}

Neste exemplo, e utilizada uma ação gerada pela pressão do vento. Devido ao comportamento estacionário que o vento possui na maior parte do tempo, é simulado um comportamento localmente estacionário do carregamento gerado por DHWT e sua comparação com o carregamento gerado por Série de Fourier, este aproximadamente estacionário. O modelo estrutural de shear building adotado possui 5 pavimentos, cada um com 4 pilares de aço e lajes de concreto.

Todos os dados são apresentados a seguir:

- Quantidade de pavimentos:

- Quantidade de pontos analisados:

- Valor de $\beta$ utilizado:

- Tensão de escoamento do aço (MPa):

- Módulo de elasticidade do aço (MPa):

- Tipos de perfis métalicos:

- Momento de Inércia dos pilares $\left(\mathrm{m}^{4}\right)$ :

- Módulo de resistência plástica $\left(\mathrm{m}^{3}\right)$ :

- Altura dos pilares por pavimento (m):

Podem-se obter as seguintes características estruturais:

- Massa das lajes (kg):

- Rigidez elástica dos pilares por pavimento $(\mathrm{N} / \mathrm{m})$ :

- Frequiências naturais da estrutura $(\mathrm{rad} / \mathrm{s})$ :

- Taxas de amortecimento da estrutura:

- Cortantes plásticos dos pilares por pavimento $(\mathrm{N})$ : $\mathrm{m}=5$

$\mathrm{N}=16384$

$\beta=1 / 6$

$\mathrm{f}_{\mathrm{y}}=250$

$\mathrm{E}=210000$

CS200x39 pavimento 1

CS150x 25 pavimentos 2 a 5

$\mathrm{I}_{1}=1.267 \times 10^{-5}$

$\mathrm{I}_{2}=\mathrm{I}_{3}=\mathrm{I}_{4}=\mathrm{I}_{5}=4.5 \times 10^{-6}$

$Z_{1}=1.92 \times 10^{-4}$;

$Z_{2}=Z_{3}=Z_{4}=Z_{5}=9.1 \times 10^{-5}$

$\ell_{1}=\ell_{2}=\ell_{3}=\ell_{4}=\ell_{5}=3 \mathrm{~m}$ $m_{1}=m_{2}=m_{3}=m_{4}=m_{4}=20000$

$k_{1}=4730133$;

$k_{2}=k_{3}=k_{4}=k_{5}=1680000$

$\omega_{i}=2.953 ; \omega_{i_{2}}=8.5691$;

$\omega_{\mathrm{i}_{3}}=13.3423 ; \omega_{\mathrm{i}_{4}}=16.7905$;

$\omega_{\mathrm{s}}=19.1421$

$\xi_{1}=0.01 ; \xi_{2}=0.01$

$\mathrm{Qpl}_{1}=128000$;

$\mathrm{Qpl}_{2}=\mathrm{Qpl}_{3}=\mathrm{Qpl}_{4}=\mathrm{Qpl}_{5}=60700$ 
Os dados necessários para a obtenção das intensidades dos carregamentos são:

- Velocidade média do vento $(\mathrm{m} / \mathrm{s})$ :

- Altura do centro de cada módulo (m):

- Altura do centro de rajada (m):

- Pressão estática em cada módulo (Pa):

- Pressão de pico em cada módulo (Pa):

- Coeficiente de arrasto em cada módulo:

- Área que atua a pressão em cada múdulo $\left(\mathrm{m}^{2}\right)$ :

- Alturas das incidências das forças:

$$
\begin{aligned}
& \mathrm{V}=50 \\
& \overline{\mathrm{z}}_{1}=2.25 ; \overline{\mathrm{z}}_{2}=6 ; \overline{\mathrm{z}}_{3}=9 ; \overline{\mathrm{z}}_{4}=12 ; \\
& \overline{\mathrm{z}}_{5}=14.25 \\
& \mathrm{Gc}=12 ; \\
& p_{\text {est } 1}=390 ; p_{\text {est } 2}=514 ; \\
& p_{\text {est } 3}=605 ; p_{\text {est } 4}=678 ; \\
& p_{\text {est5 }}=741 \\
& p_{\text {pic } 1}=1376 ; p_{\text {pic } 2}=1581 ; \\
& p_{\text {pic3 }}=1714 ; p_{\text {pic } 4}=1815 ; \\
& p_{\text {pic5 }}=1898 \\
& \mathrm{Ca}_{1}=\mathrm{Ca}_{2}=\mathrm{Ca}_{3}=0.9 ; \\
& \mathrm{Ca}_{4}=\mathrm{Ca}_{5}=0.9 \\
& A_{1}=27 ; A_{2}=A_{3}=A_{4}=18 ; \\
& A_{5}=9 \\
& \mathrm{z}_{1}=3 ; \mathrm{z}_{2}=6 ; \mathrm{z}_{3}=9 ; \mathrm{z}_{4}=12 ; \\
& \mathrm{z}_{5}=15
\end{aligned}
$$

Onde a Figura 6.6 mostra o posicionamento das forças geradas pelo vento aplicadas na estrutura:

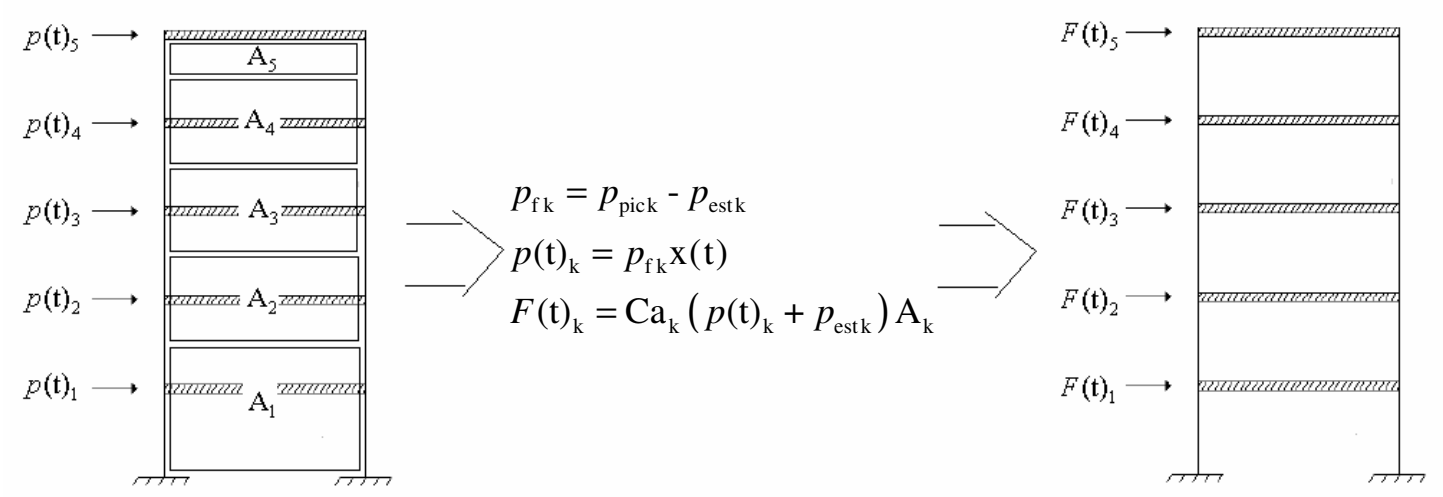

Figura 6.6. Forças devido ao vento na estrutura. 
As características do sinal gerado por DHWT são:

- Tempo do sinal gerado (s):

- Incremento de tempo (s):

- Variação da janela tempo-frequência:

- Fator de variação:

- Quantidade de níveis:

- Nível que possui a freqüência fundamental:

- Menor frequiência do sinal gerado ( $\mathrm{rad} / \mathrm{s})$ :

- Maior freqüência sem repetição do espectro ( $\mathrm{rad} / \mathrm{s})$ :
$\mathrm{T}=600$

$\Delta \mathrm{t}=0.03662$

Geométrica

$\mathrm{q}=2$

$\mathrm{n}=14$

jf $=9$

$\omega^{\mathrm{w}-}=0.007694$

$\omega_{\mathrm{n}-1}^{\mathrm{w}+}=63.0339$

As características do sinal gerado por Série de Fourier são:

- Tempo do sinal gerado (s):

- Incremento de tempo (s):

- Variação do intervalo de frequência:

- Fator de variação:

- Quantidade de harmônicos:

- Nível que possui a frequiência fundamental:

- Menor frequiência do sinal gerado ( $\mathrm{rad} / \mathrm{s})$ :

- Maior freqüência sem repetição do espectro (rad/s):

$$
\begin{aligned}
& \mathrm{T}=600 \\
& \Delta \mathrm{t}=0.03662
\end{aligned}
$$

Geométrica

$\mathrm{q}=1.12175$

$\mathrm{n}=60$

if $=34$

$\omega_{1}^{\mathrm{F}-}=0.007796$

$\omega_{\mathrm{n}}^{\mathrm{F}+}=63.0659$

As forças nos nós da estrutura devido à pressão de vento atuam na direção de menor inércia dos perfis metálicos, onde todos os perfis são considerados de massa desprezível. O espectro de frequiência utilizado é o (5.97) para ação gerada, e a função de tendência, usada para a ação gerada por DHWT de comportamento não estacionário desta vez é uma reta constante, com o intuito de simular um comportamento estacionário. Estes podem ser descritos, respectivamente:

$$
\begin{aligned}
& \frac{\mathrm{W}_{\mathrm{v}}(f)}{\sigma_{\mathrm{v}}{ }^{2}}=\frac{4 \mathrm{v}^{2}}{f\left(1+\mathrm{v}^{2}\right)^{\frac{4}{3}}} \quad \text { para } \quad \mathrm{v}=\frac{1220 f}{\mathrm{~V}} \\
& \mathrm{f}(\mathrm{t})=1
\end{aligned}
$$


Na Figura 6.7(a) e 6.7(b), podem ser vistos (6.32) e (6.33):

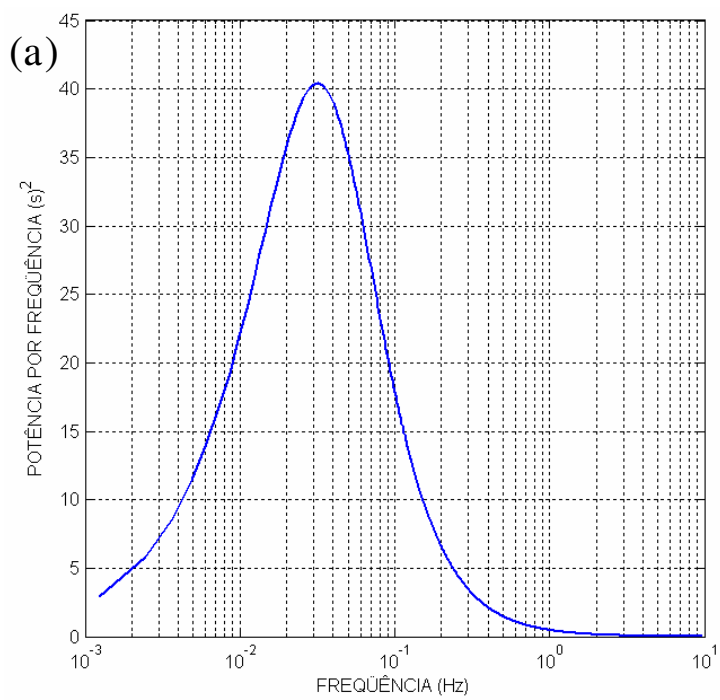

(b)

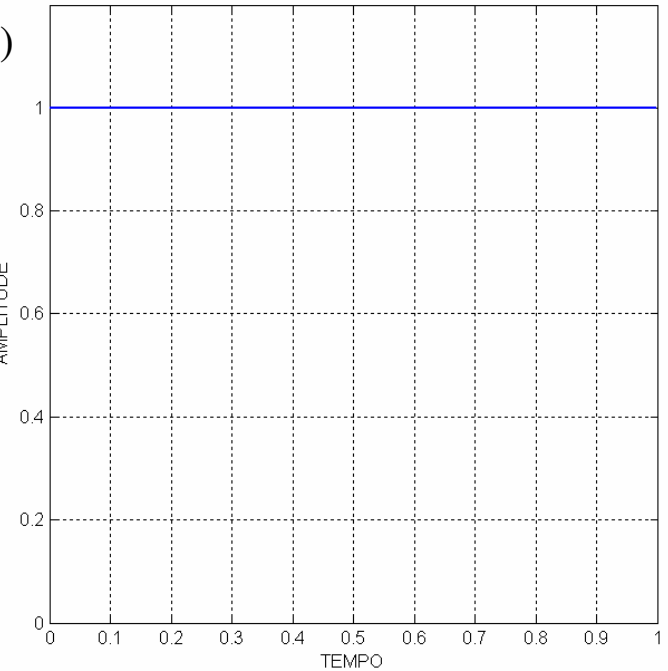

Figura 6.7. São as curvas: (a) Espectro de frequiência; (b) Função de tendência.

Em posse destes dados, os dois tipos de ações são gerados na forma normalizada levando em consideração a correlação espacial devido ao efeito da rajada para cada ação em um nó do shear building.

Para a comparação dos dois modelos, devido ao fato dos sinais das pressões do vento serem gerados com a ajuda de uma função pseudo-aleatória, são analisados os escalogramas, através do uso da transformada da wavelet harmônica; e os espectros de potência, através do uso da transformada de Fourier. Todas estas são oriundas dos sinais, mostrados a seguir:

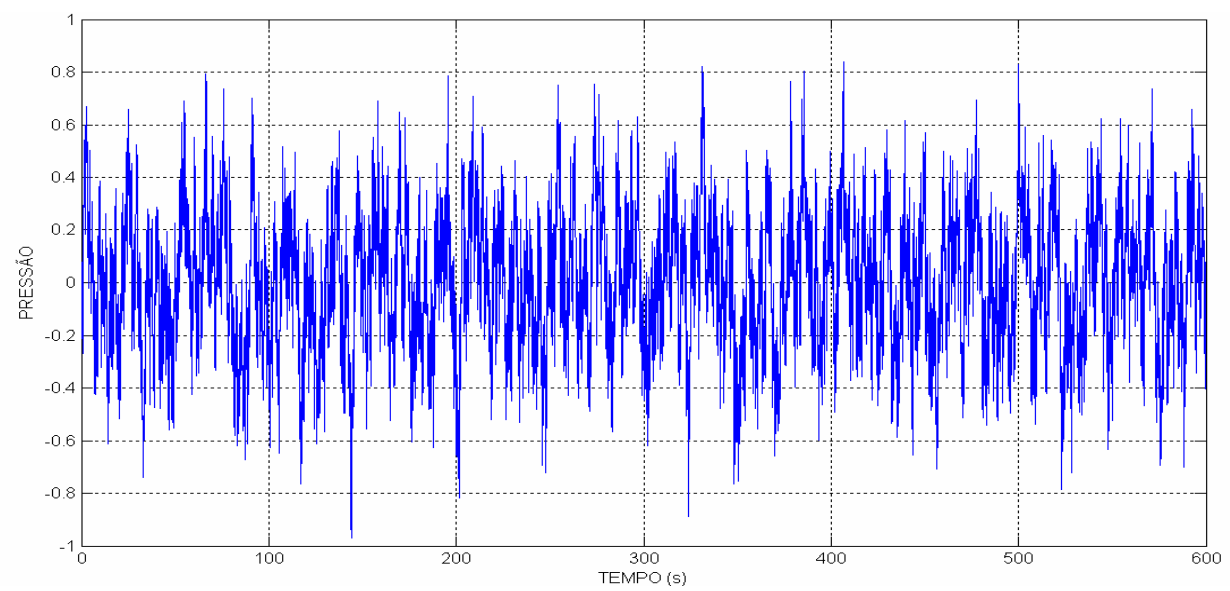

Figura 6.8. Sinal da pressão normalizada do vento atuante no centro de rajada, com comportamento localmente estacionário, gerado a partir de DHWT. 


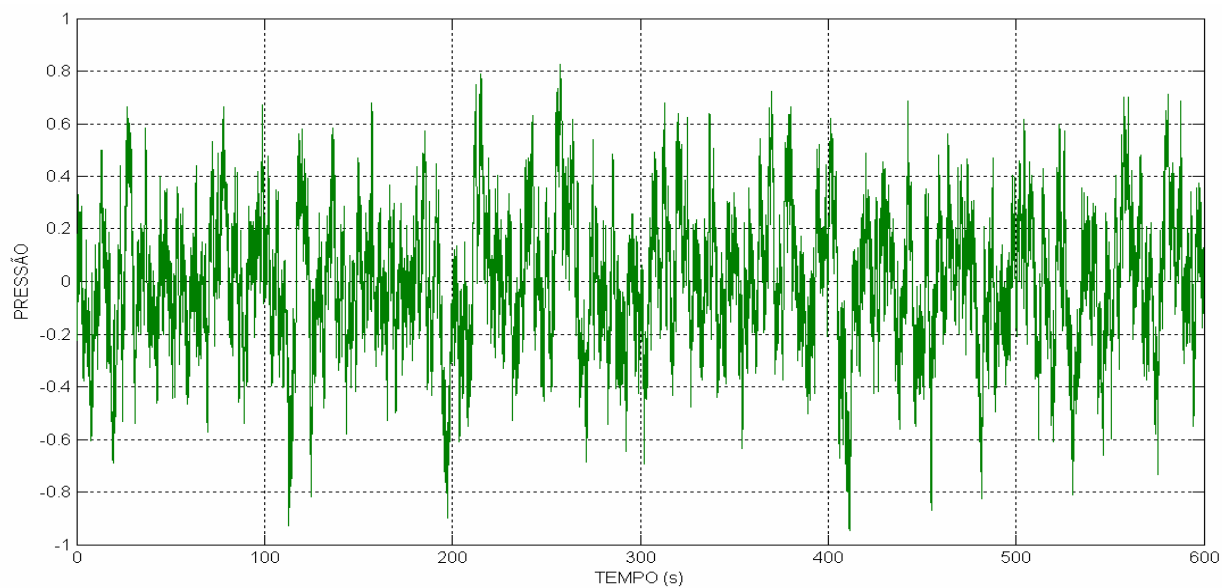

Figura 6.9. Sinal da pressão normalizada do vento atuante no centro de rajada, com comportamento próximo do estacionário, gerado a partir de Série de Fourier.

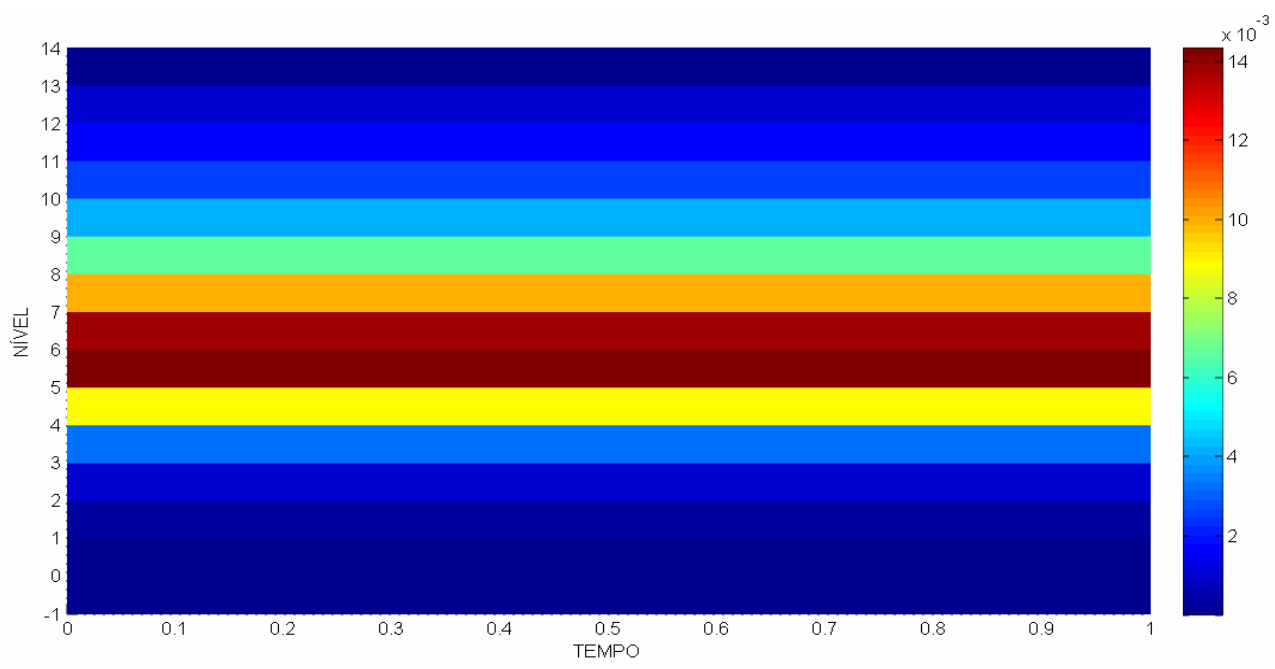

Figura 6.10. Escalograma da pressão normalizada do vento atuante no centro de rajada, com comportamento localmente estacionário, gerado a partir de DHWT.

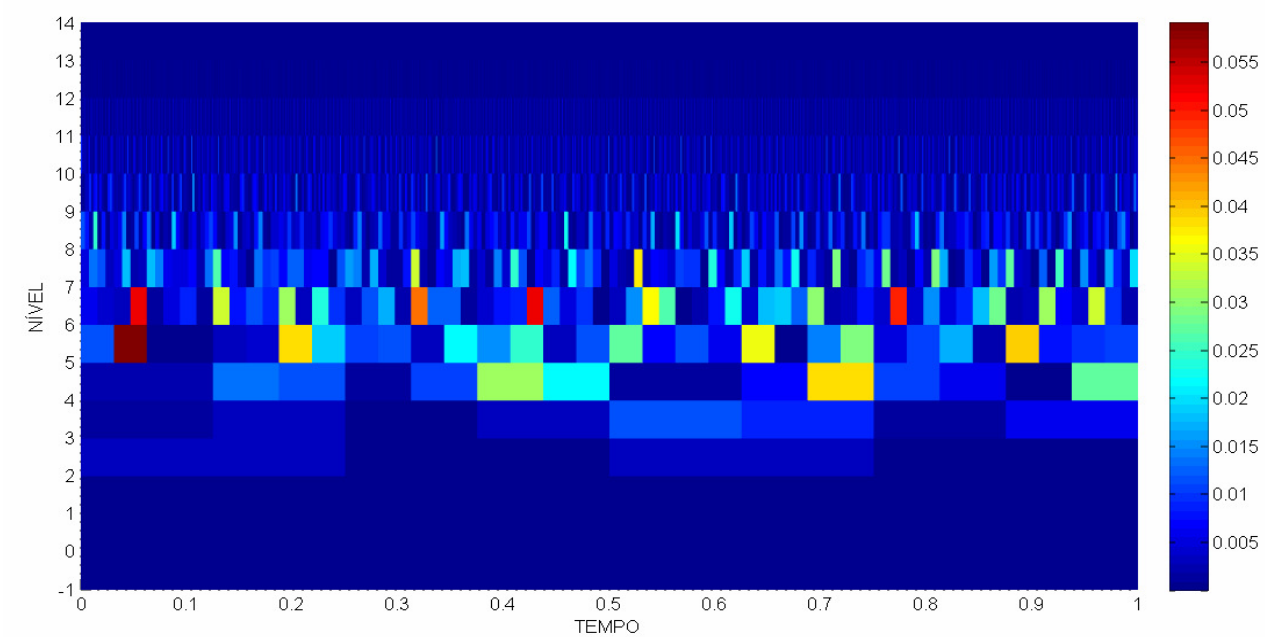

Figura 6.11. Escalograma da pressão normalizada do vento atuante no centro de rajada, com comportamento próximo do estacionário, gerado a partir de Série de Fourier. 


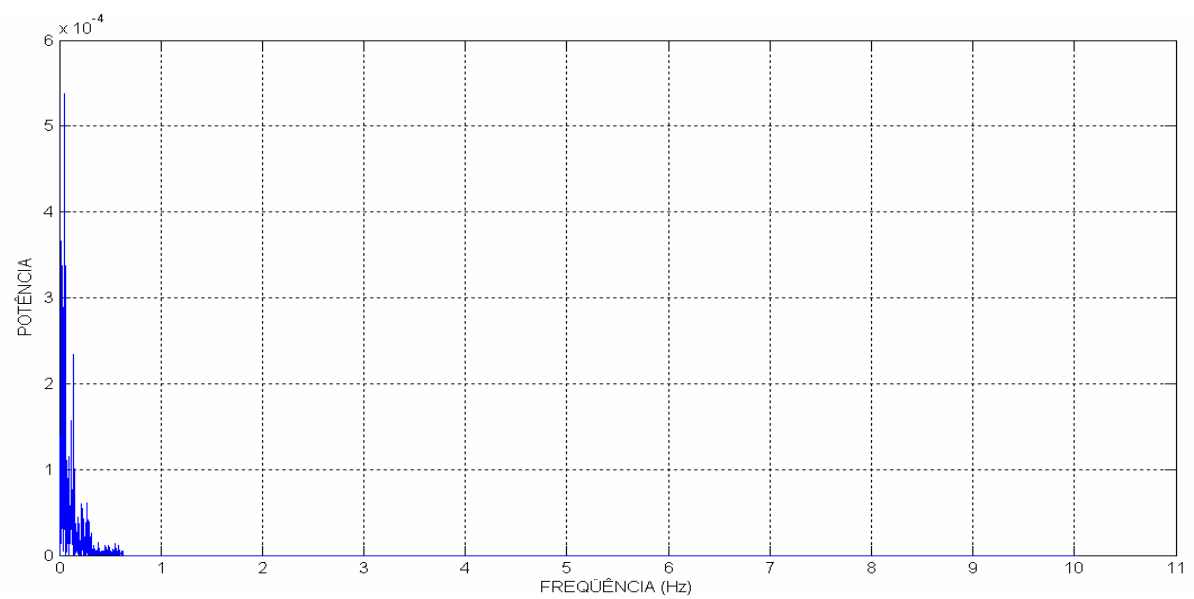

Figura 6.12. Espectro de potência da pressão normalizada do vento atuante no centro de rajada, com comportamento localmente estacionário, gerado a partir de DHWT.

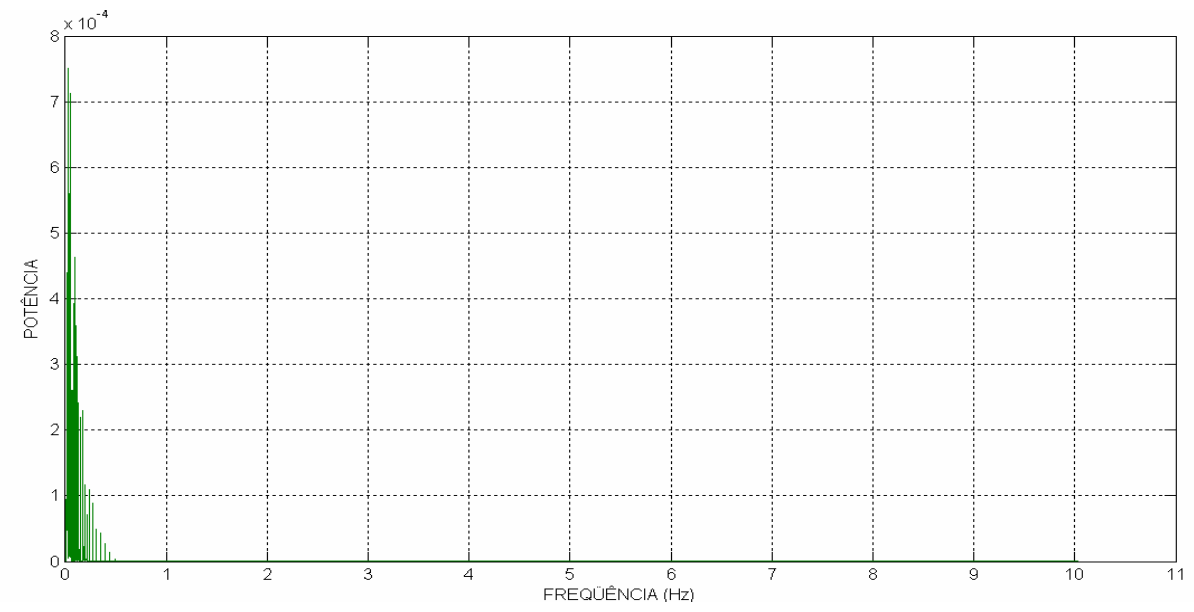

Figura 6.13. Espectro de potência da pressão normalizada do vento atuante no centro de rajada, com comportamento próximo do estacionário, gerado a partir de Série de Fourier.

Agora, convertendo estes sinais em carregamento e os aplicando, ambos pelos processos descritos anteriormente, e realizando a análise dinâmica incremental pelo método de Newmark, podemos obter a resposta dinâmica da estrutura para as vinte séries de ações de cada modelo.

É apresentada na Figura 6.13, as respostas estruturais dos valores extremos dos deslocamentos de $u_{5}$, para um comportamento elástico linear e elasto-plástico perfeito com ações geradas a partir de DHWT, e na Figura 6.14, para um comportamento elástico linear elasto-plástico perfeito com ações geradas a partir de Série de Fourier: 


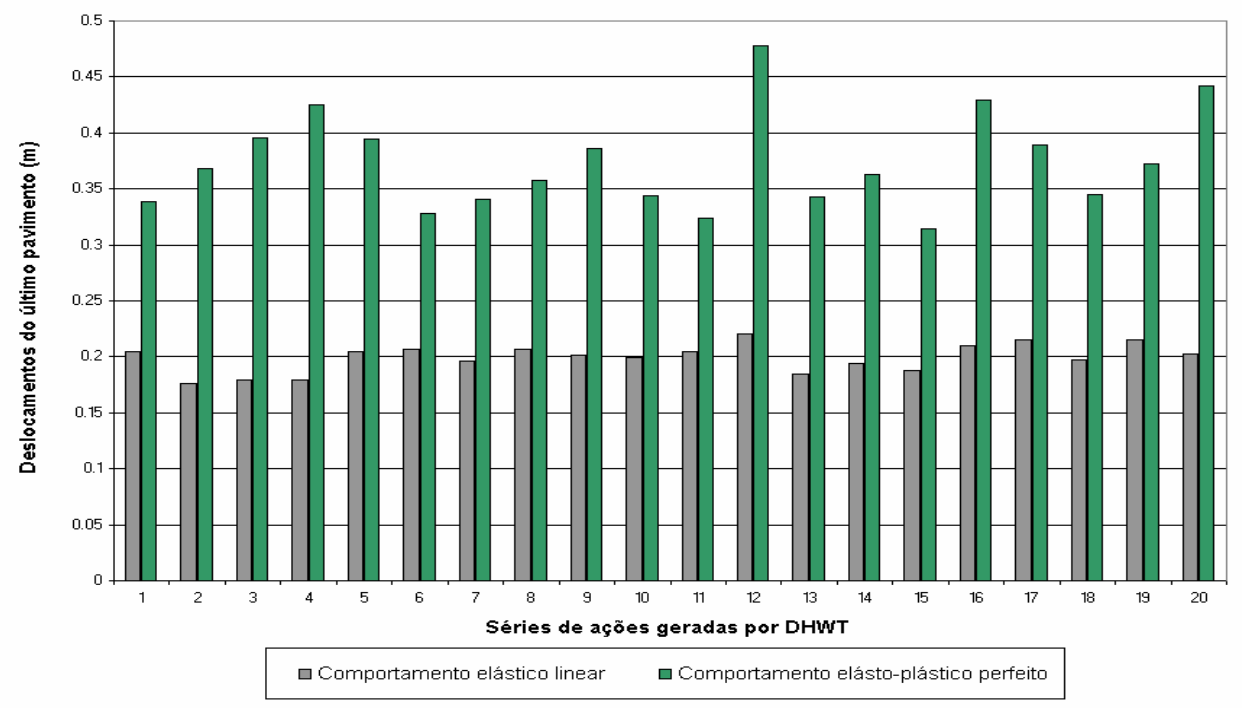

Figura 6.14. Deslocamentos do último pavimento devido a ações geradas por DHWT.

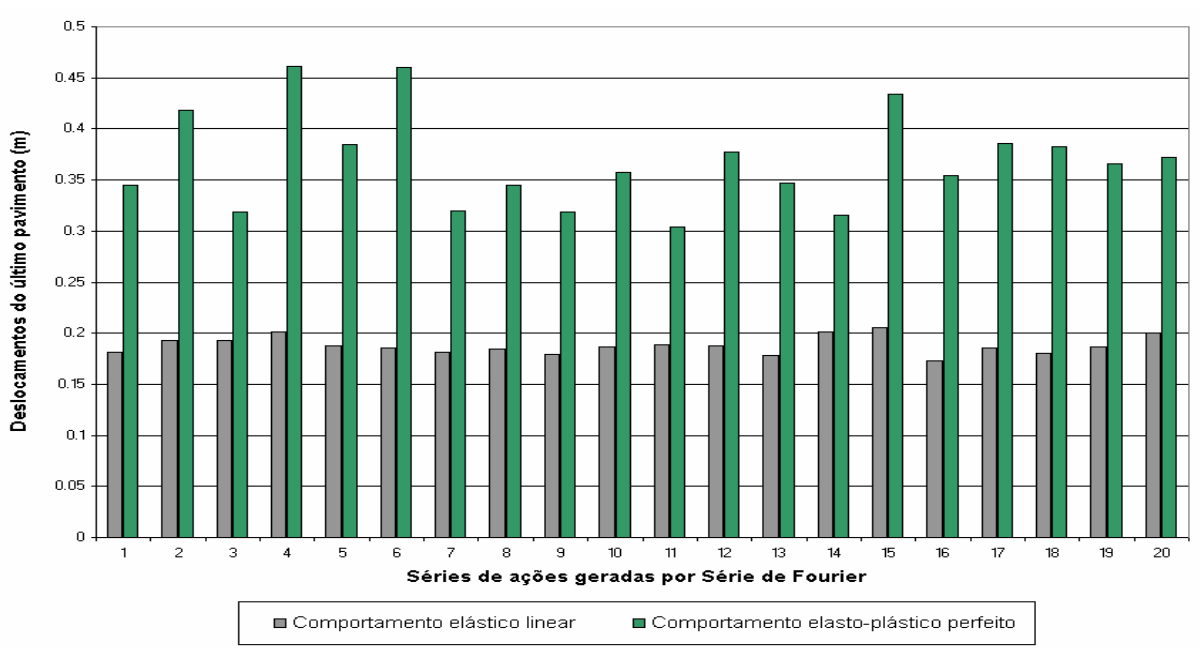

Figura 6.15. Deslocamentos do último pavimento devido a ações geradas por Série de Fourier.

São escolhidos então os deslocamentos de $u_{5}$ que apresentam os valores próximos dos valores característicos das séries de cada modelo, este valor representa $95 \%$ de probabilidade dos valores de outras ações serem menor que ele. 
Tabela 6.1. Dados estatísticos dos deslocamentos sofridos pela estrutura devido às séries dos dois modelos empregados.

\begin{tabular}{|l|r|l|r|}
\hline \multicolumn{5}{|c|}{ Séries de ações geradas a partir de DHWT (m) } \\
\hline \multicolumn{3}{|c|}{ Comportamento elástico linear } & \multicolumn{1}{c|}{ Comportamento elasto-plástico perfeito } \\
\hline $\mathrm{E}[\mathrm{x}]$ & 0.1993 & $\mathrm{E}[\mathrm{x}]$ & 0.3739 \\
\hline$\sigma_{\mathrm{x}}$ & 0.01259 & $\sigma_{\mathrm{x}}$ & 0.04358 \\
\hline $\mathrm{E}[\mathrm{x}]+1.65 \sigma_{\mathrm{x}}$ & 0.2201 & $\mathrm{E}[\mathrm{x}]+1.65 \sigma_{\mathrm{x}}$ & 0.4458 \\
\hline \multicolumn{3}{|c|}{ Séries de ações geradas a partir de Série de Fourier (m) } \\
\hline \multicolumn{3}{|c|}{ Comportamento elástico linear } & Comportamento elasto-plástico perfeito \\
\hline $\mathrm{E}[\mathrm{x}]$ & 0.1881 & $\mathrm{E}[\mathrm{x}]$ & 0.3683 \\
\hline$\sigma_{\mathrm{x}}$ & 0.008689 & $\sigma_{\mathrm{x}}$ & 0.04638 \\
\hline $\mathrm{E}[\mathrm{x}]+1.65 \sigma_{\mathrm{x}}$ & 0.2024 & $\mathrm{E}[\mathrm{x}]+1.65 \sigma_{\mathrm{x}}$ & 0.4448 \\
\hline
\end{tabular}

Como pode ser observado, para o modelo desenvolvido neste trabalho, a série de número 20 possui valores próximos dos valores característicos, e para o modelo gerado por Série de Fourier, a série de número 15 apresenta os valores mais próximos dos valores característicos. Percebe-se que para ambos os modelos de geração de ações, seus valores são muito próximos, isto é esperado, pois, foi utilizada uma função de tendência de valor constante para a representação de um comportamento localmente estacionário. 


\subsection{EXEMPLO 3}

Neste exemplo e utilizada uma ação devido a sismo, baseado no sinal da componente horizontal da aceleração do terremoto de Takoshi-oki, com magnitude 7.9 da escala Richter, ocorrido na cidade de Hachinohe, em 16 de maio de 1968 no Japão. O modelo estrutural de shear building adotado possui 4 pavimentos, cada um com 4 pilares de aço e lajes de concreto.

Todos os dados são apresentados a seguir:

- Quantidade de pavimentos:

- Valor da aceleração máxima $\left(\mathrm{m} / \mathrm{s}^{2}\right)$ :

- Quantidade de pontos analizados:

- Valor de $\beta$ utilizado:

- Taxa de amortecimento do solo:

- Freqüência fundamental do solo ( $\mathrm{rad} / \mathrm{s})$ :

- Tensão de escoamento do aço (MPa):

- Módulo de elasticidade do aço (MPa):

- Tipos de perfis métalicos:

- Momento de Inércia dos pilares $\left(\mathrm{m}^{4}\right)$ :

- $\quad$ Módulo de resistência plástica $\left(\mathrm{m}^{3}\right)$ :

- $\quad$ Altura dos pilares por pavimento (m): $\mathrm{m}=4$

$\ddot{u}_{\text {max }}=2.3$

$\mathrm{N}=4096$

$\beta=1 / 6$

$\xi_{\mathrm{g}}=0.7$

$\omega_{\mathrm{g}}=2.2 \mathrm{rad} / \mathrm{s}$

$\mathrm{f}_{\mathrm{y}}=250$

$\mathrm{E}=210000$

CS200x39 pavimentos 1 e 2

CS150x 25 pavimentos 3 e 4

$\mathrm{I}_{1}=\mathrm{I}_{2}=1.267 \times 10^{-5}$;

$\mathrm{I}_{2}=\mathrm{I}_{3}=4.5 \times 10^{-6}$

$Z_{1}=Z_{2}=9.1 \times 10^{-5}$;

$Z_{1}=Z_{2}=1.92 \times 10^{-5}$

$\ell_{1}=\ell_{2}=\ell_{3}=\ell_{4}=3 \mathrm{~m}$

Podem-se obter as seguintes características estruturais:

- $\quad$ Massa das lajes (kg):

- Rigidez elástica dos pilares por pavimento $(\mathrm{N} / \mathrm{m})$ :

- Frequiências naturais da estrutura ( $\mathrm{rad} / \mathrm{s})$ :

- Taxas de amortecimento da estrutura:

- Cortantes plásticos dos pilares por pavimento $(\mathrm{N})$ : $m_{1}=m_{2}=m_{3}=m_{4}=8000$

$k_{1}=k_{2}=4730133$;

$k_{3}=k_{4}=1680000$

$\omega_{\mathrm{f}}=6.9453 ; \quad \omega_{\mathrm{f}_{2}}=17.7394$;

$\omega_{\mathrm{f}_{3}}=24.9895 ; \omega_{\mathrm{f}_{4}}=40.3287$

$\xi_{1}=0.01 ; \xi_{2}=0.01$

$\mathrm{Qpl}_{1}=\mathrm{Qpl}_{2}=128000$

$\mathrm{Qpl}_{3}=\mathrm{Qpl}_{4}=60700$ 
As características do sinal de referência utilizado são:

- Tempo do sinal original (s):

$\mathrm{T}=36$

- Tempo do sinal modificado:

$\mathrm{T}=40.95$

- Incremento de tempo (s):

$\Delta \mathrm{t}=0.01$

- Menor freqüência do sinal original $(\mathrm{Hz})$ :

0.0278

- Maior frequiência sem repetição do espectro $(\mathrm{Hz}): \quad 50$

- Maior freqüência do sinal original $(\mathrm{Hz})$ : 100

- Menor freqüência do sinal modificado (Hz): 0.0244

- Maior freqüência sem repetição do espectro $(\mathrm{Hz}): \quad 50$

- Maior frequiência do sinal modificado $(\mathrm{Hz})$ : 100

As características do sinal gerado por DHWT são:

- Tempo do sinal gerado (s):

$\mathrm{T}=40.95$

- Incremento de tempo (s):

$\Delta \mathrm{t}=0.01$

- Variação da janela tempo-frequência:

Geométrica

- Fator de variação:

$\mathrm{q}=2$

- Quantidade de níveis:

$\mathrm{n}=12$

- Nível que possui a frequiência fundamental:

jf $=6$

- Menor frequiência do sinal gerado ( $\mathrm{rad} / \mathrm{s})$ :

$\omega^{\mathrm{w}-}=0.1448$

- Maior freqüência sem repetição do espectro $(\mathrm{rad} / \mathrm{s})$ :

$\omega^{\mathrm{w}+}{ }_{\mathrm{n}-1}=296.6173$

As características do sinal gerado por Série de Fourier são:

- Tempo do sinal gerado (s):

$\mathrm{T}=40.95$

- Incremento de tempo (s):

$\Delta \mathrm{t}=0.01$

- Variação do intervalo de frequência:

Geométrica

- Fator de variação:

$\mathrm{q}=1.0894$

- Quantidade de harmônicos:

$\mathrm{n}=60$

- Nível que possui a frequiência fundamental:

if $=19$

- Menor frequiência do sinal gerado ( $\mathrm{rad} / \mathrm{s})$ :

$\omega^{\mathrm{F}-}=0.1565$

- Maior freqüência sem repetição do espectro ( $\mathrm{rad} / \mathrm{s})$ :

$\omega_{\mathrm{n}}^{\mathrm{F}+}=296.5877$ 
A aceleração do solo atua na direção de menor inércia dos perfis metálicos, onde todos os perfis são considerados de massa desprezível. O espectro de freqüência utilizado é o (5.97) para ambas as ações geradas, e a função de tendência, usada para a ação gerada de comportamento não estacionário, é uma composição entre duas funções exponencis. Estes podem ser descritos, respectivamente:

$$
\begin{aligned}
& \frac{\mathrm{W}_{a c}(\omega)}{\sigma_{a c}{ }^{2}}=\frac{4 \xi_{\mathrm{g}}}{\omega_{\mathrm{f}} \pi\left(1+4 \xi_{\mathrm{g}}{ }^{2}\right)} \frac{\mathrm{r}_{\omega}\left(1+4 \xi_{\mathrm{g}}{ }^{2} \mathrm{r}_{\omega}{ }^{2}\right)}{\left(\left(1-\mathrm{r}_{\omega}{ }^{2}\right)^{2}+4 \xi_{\mathrm{g}}{ }^{2} \mathrm{r}_{\omega}{ }^{2}\right)} \quad \text { para } \quad \mathrm{r}_{\omega}=\left(\frac{\omega}{\omega_{\mathrm{g}}}\right) \\
& \mathrm{f}(\mathrm{t})=0.828\left(\mathrm{e}^{-1.3(\mathrm{t}-0.1)}+0.602 \mathrm{e}^{-\frac{16}{3}(\mathrm{t}-0.55)}\right)
\end{aligned}
$$

Na figura 6.16(a) e 6.16(b), podem ser vistos (6.34) e (6.36):
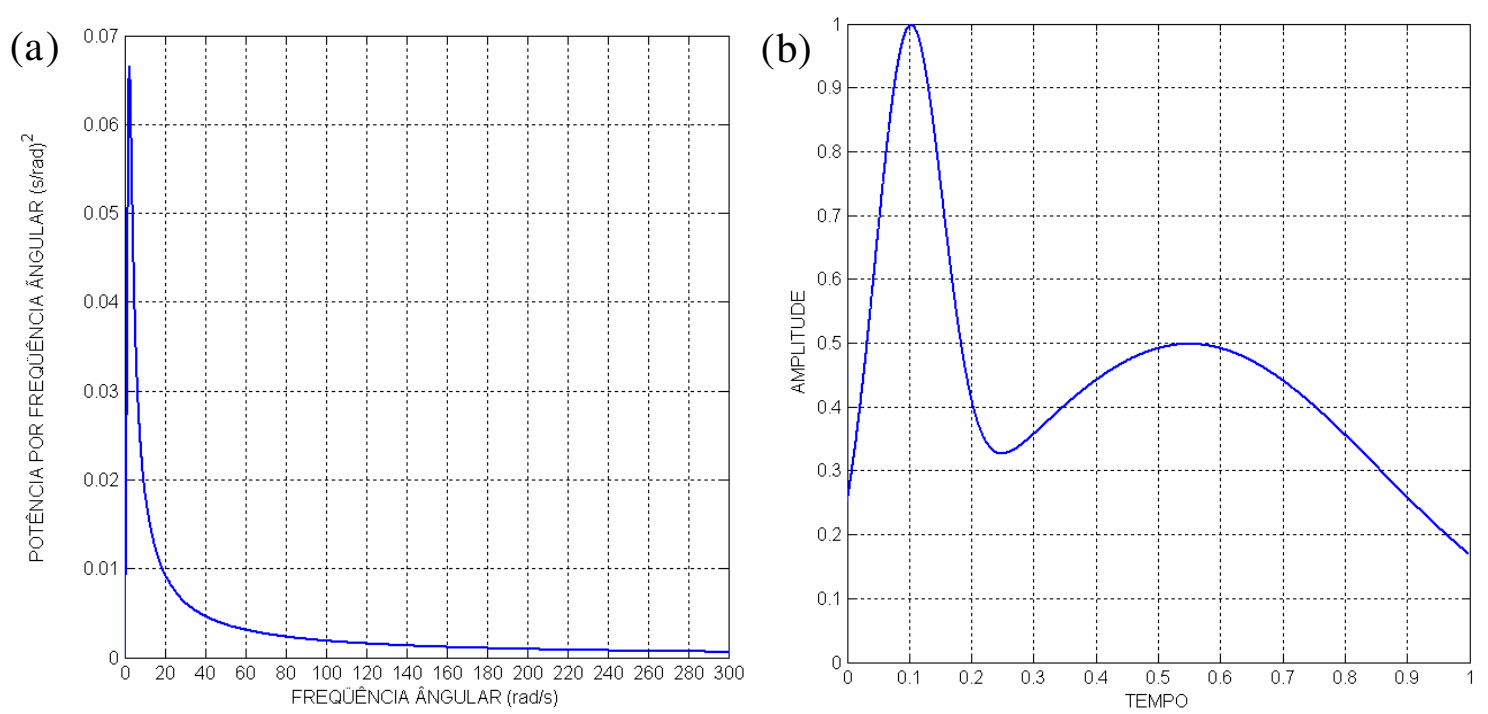

Figura 6.16. São as curvas: (a) Espectro de freqüência; (b) Função de tendência.

Em posse desses dados, os dois tipos de ações são geradas na forma normalizada e então multiplicadas por valores que possuam amplitudes máximas equivalentes a amplitude máxima da ação original, estas duas ações, juntamente com a ação original são mostradas nas Figuras 6.17, 6.18 e 6.19.

Para a comparação dos três modelos, devido ao fato dos acelerogramas serem gerados com a ajuda de uma função pseudo-aleatória, são analisados os escalogramas, através do uso da transformada da wavelet harmônica; e do espectro de potência, através do uso da transformada de Fourier. Todas estas são oriundas dos acelerogramas, mostrados a seguir: 


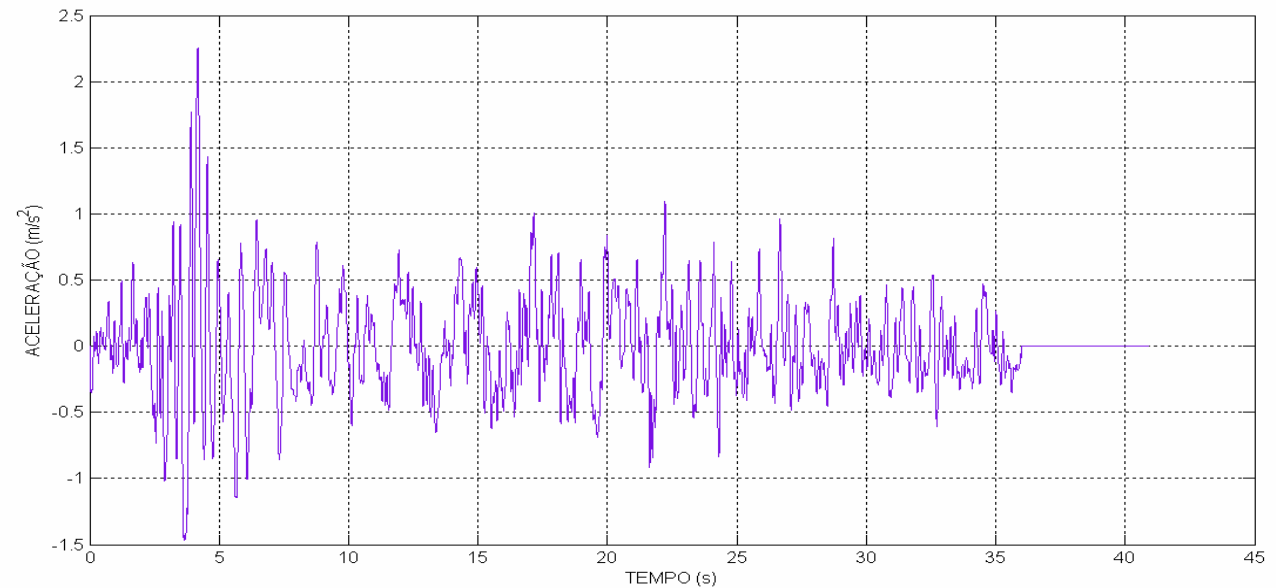

Figura 6.17. Acelerograma do terremoto ocorrido na cidade de Hachinohe, em 1968 no Japão.

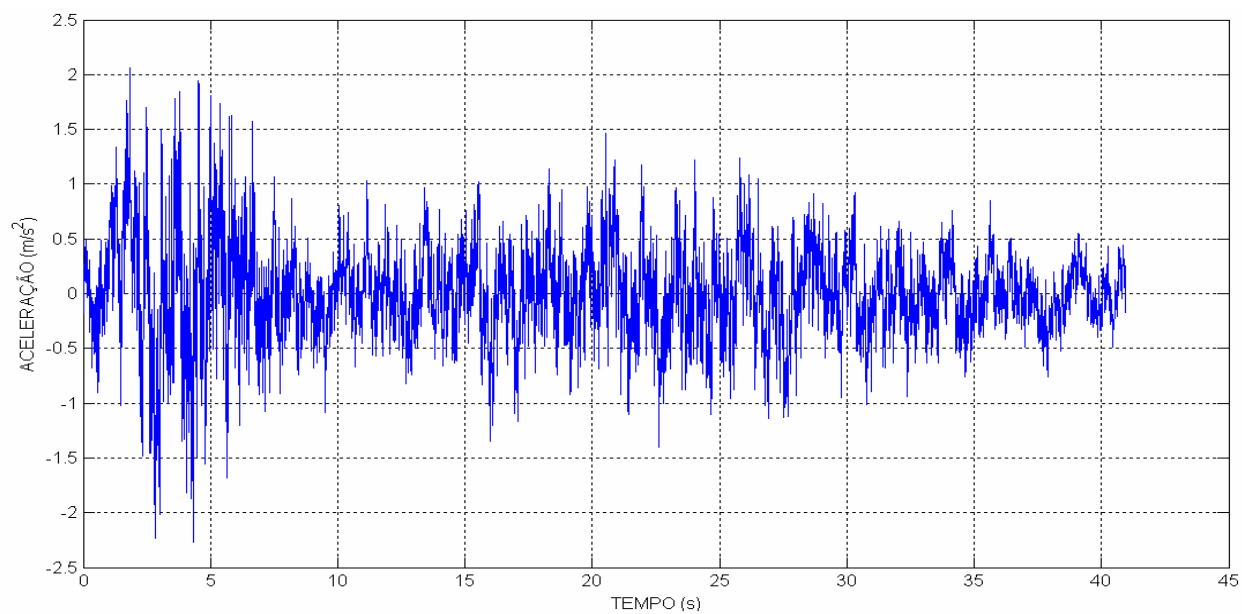

Figura 6.18. Acelerograma com comportamento não estacionário, gerado pelo a partir de DHWT.

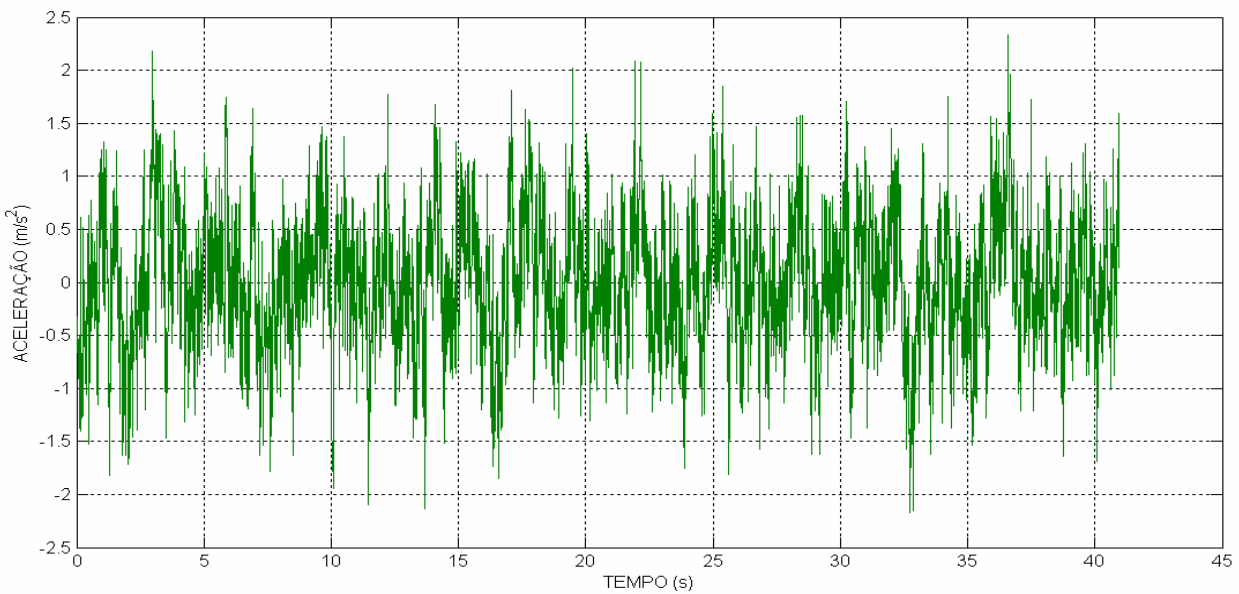

Figura 6.19. Acelerograma com comportamento estacionário, gerado a partir de Série de Fourier. 


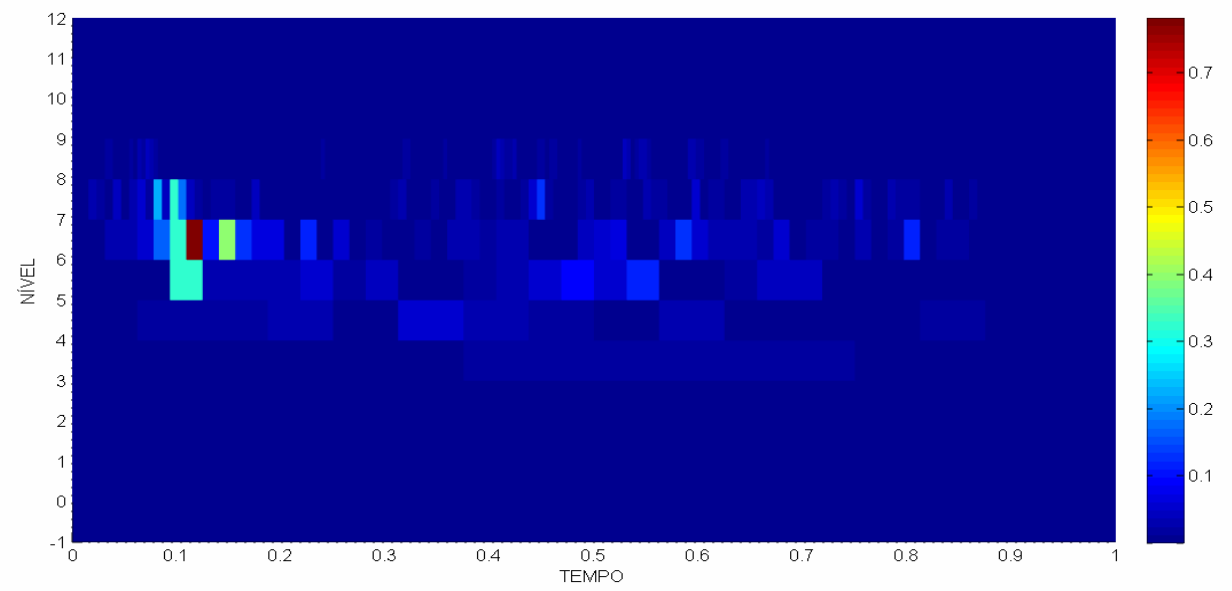

Figura 6.20. Escalograma do terremoto ocorrido na cidade de Hachinohe, em 1968 no Japão, baseado em seu acelerograma.

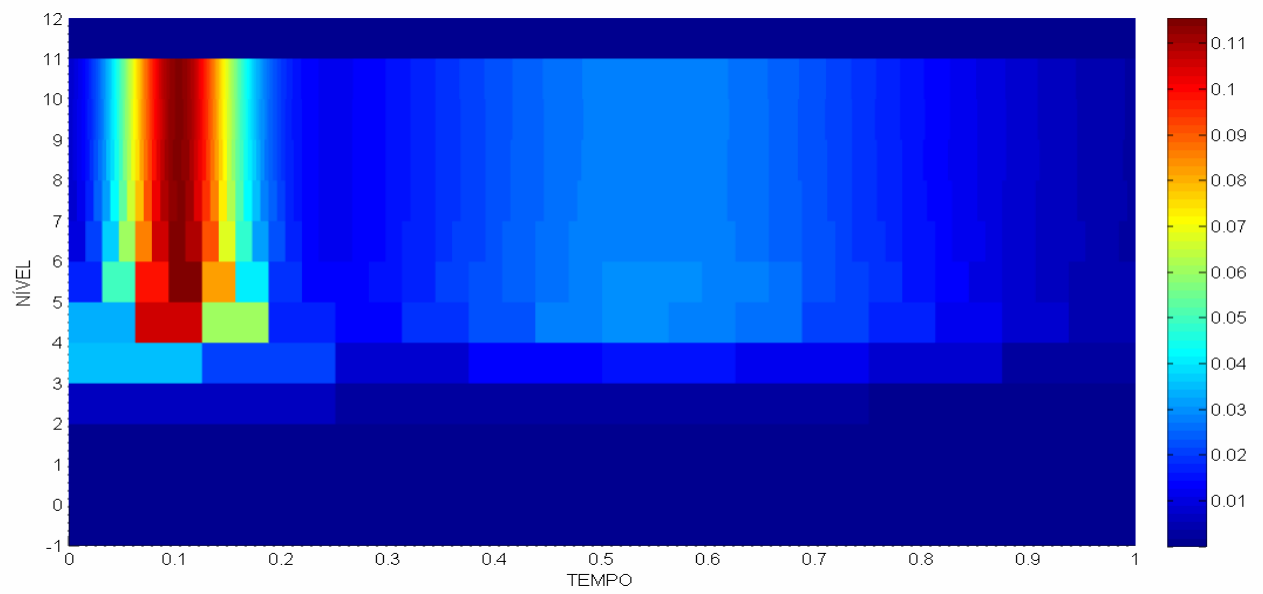

Figura 6.21. Escalograma do acelerograma com comportamento não estacionário, gerado a partir de DHWT.

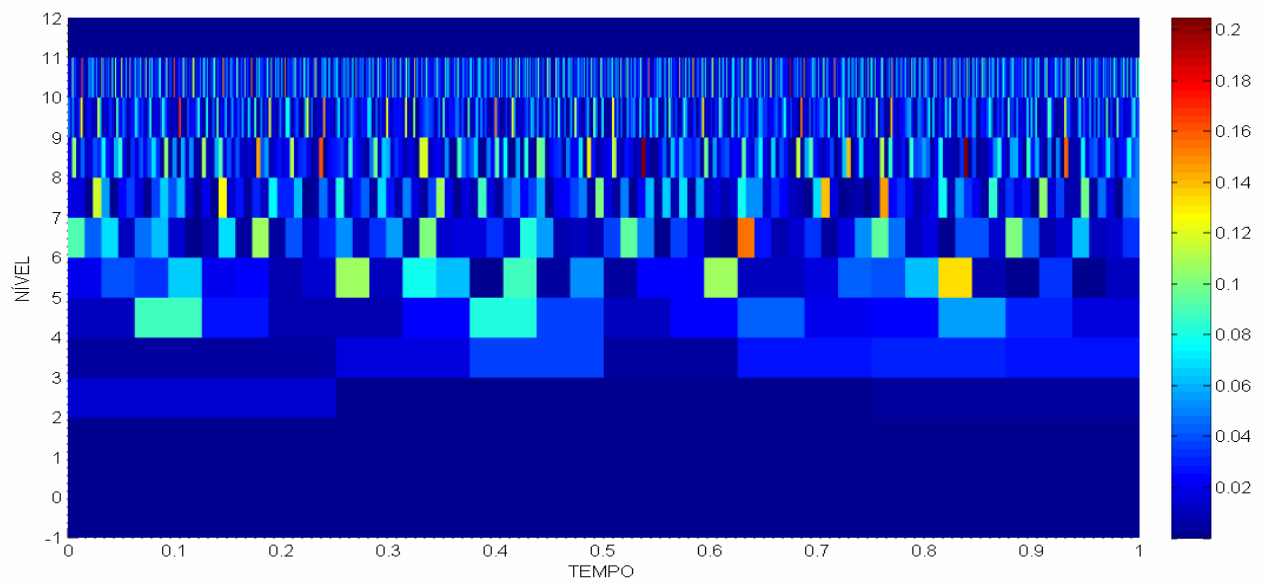

Figura 6.22. Escalograma do acelerograma com comportamento aproximadamente estacionário, gerado a partir de Série de Fourier. 


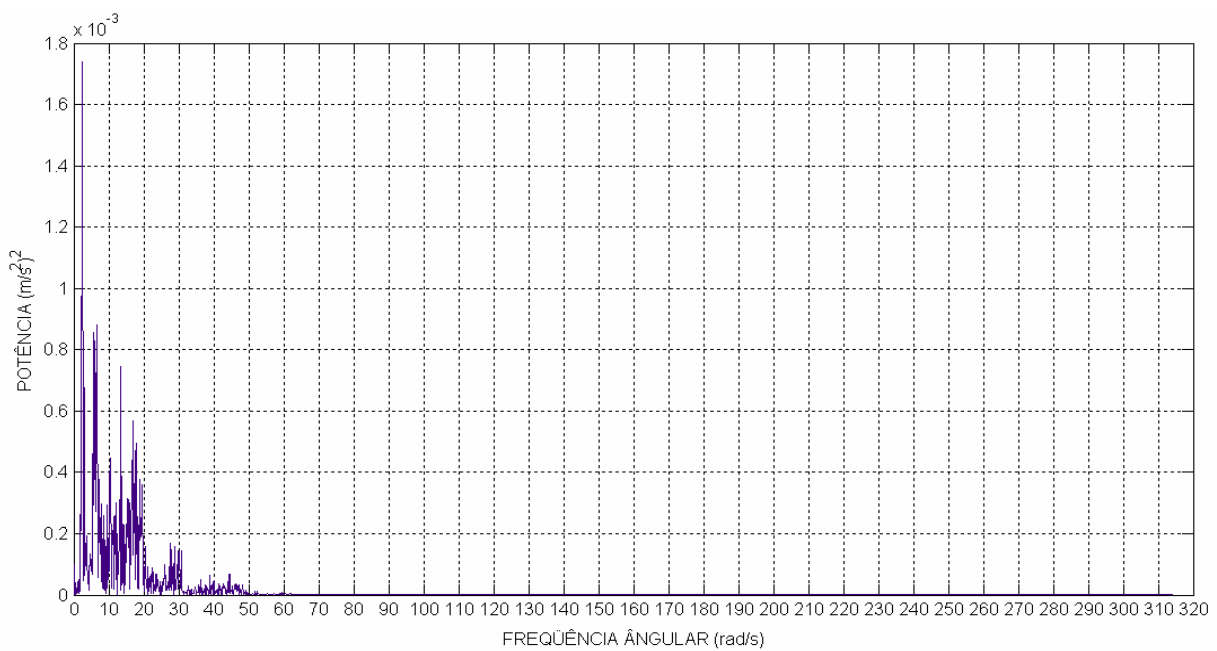

Figura 6.23. Espectro de potência do terremoto ocorrido na cidade de Hachinohe, em 1968 no Japão, baseado em seu acelerograma.

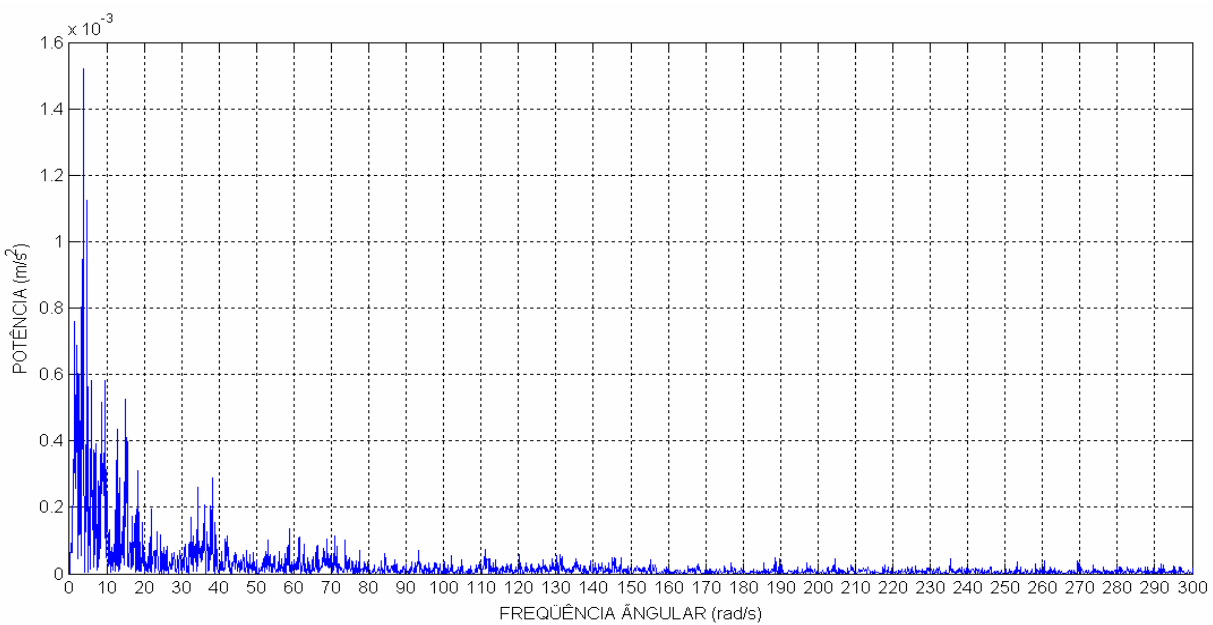

Figura 6.24. Espectro de potência do acelerograma com comportamento não estacionário, gerado a partir de DHWT.

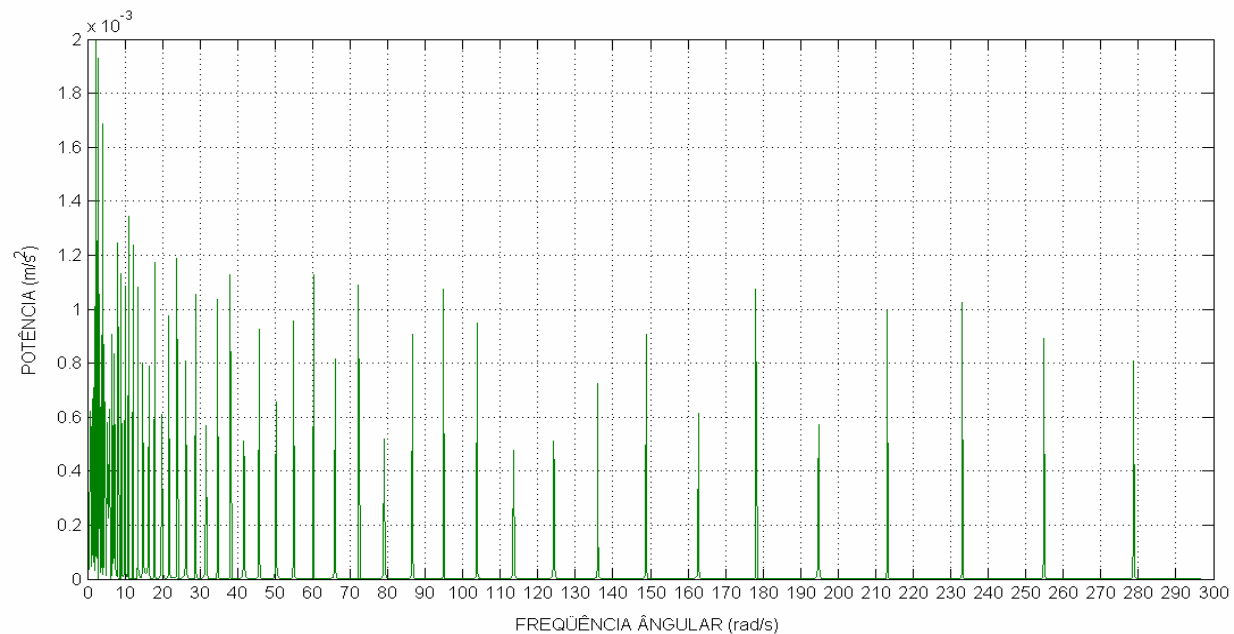

Figura 6.25. Espectro de potência do acelerograma com comportamento aproximadamente estacionário, gerado a partir de Série de Fourier. 
Em posse destes dados, são então analisados os parâmetros estatísticos, como média e desvio padrão, e assim, são calculados seus erros relativos, em relação ao sismo ocorrido em Hachinohe.

Tabela 6.2. Comparação do Escalograma e do Espectro de potência dos dois métodos, com relação ao Escalograma e Espectro de Potência do sismo em Hachinohe.

\begin{tabular}{|c|c|c|c|c|c|c|}
\hline Sinal & $\mathrm{E}[\mathrm{x}]$ & $\begin{array}{c}\text { Erro(E[x]) } \\
\%\end{array}$ & $\sigma_{\mathrm{x}}$ & $\begin{array}{c}\text { Erro }\left(\sigma_{\mathrm{x}}\right) \\
\%\end{array}$ & $\mathrm{E}[\mathrm{x}] \mathrm{E}[\mathrm{y}]$ & $\sigma_{\mathrm{xy}}$ \\
\hline \multicolumn{7}{|c|}{ ESCALOGRAMA } \\
\hline Hachinohe & $4.3388 \mathrm{E}-3$ & & $2.6194 \mathrm{E}-2$ & & & \\
\hline DHWT & $1.4982 \mathrm{E}-2$ & 245.3028 & $3.8803 \mathrm{E}-2$ & 48.1309 & $3.0655 \mathrm{E}-4$ & 0.23765 \\
\hline SF & $5.5794 \mathrm{E}-2$ & 1185.9 & $5.1852 \mathrm{E}-2$ & 97.947 & $2.4438 \mathrm{E}-4$ & $1.6903 \mathrm{E}-3$ \\
\hline \multicolumn{7}{|c|}{ ESPECTRO DE POTÊNCIA } \\
\hline Hachinohe & $1.6835 \mathrm{E}-5$ & \multicolumn{1}{|c|}{$8.4177 \mathrm{E}-5$} & & & \\
\hline DHWT & $4.0235 \mathrm{E}-5$ & 139.01 & $1.2136 \mathrm{E}-4$ & 44.1688 & $4.6257 \mathrm{E}-9$ & 0.3865 \\
\hline SF & $5.33 \mathrm{E}-5$ & 216.61 & $2.6702 \mathrm{E}-4$ & 217.2113 & $7.7929 \mathrm{E}-9$ & 0.3068 \\
\hline
\end{tabular}

Obs:SF-Série de Fourier.

Como pode ser observado, embora o erro de ambos os modelos de ações sejam grandes, o modelo que usa DHWT, possui erros bem menores em comparação ao modelo que usa Série de Fourier, e isto é bastante notório no valor da covariância do escalograma.

Agora, aplicando-se estas ações segundo o processo descrito anteriormente, e realizando a análise dinâmica incremental pelo método de Newmark, podemos obter a resposta dinâmica da estrutura para vinte séries de ações de cada modelo.

É apresentada na Figura 6.26, as respostas estruturais dos valores extremos dos deslocamentos de $u_{4}$, para um comportamento elástico linear e elasto-plástico perfeito com ações geradas a partir de DHWT, e na Figura 6.27, para um comportamento elástico linear e elasto-plástico perfeito com ações geradas a partir de Série de Fourier: 


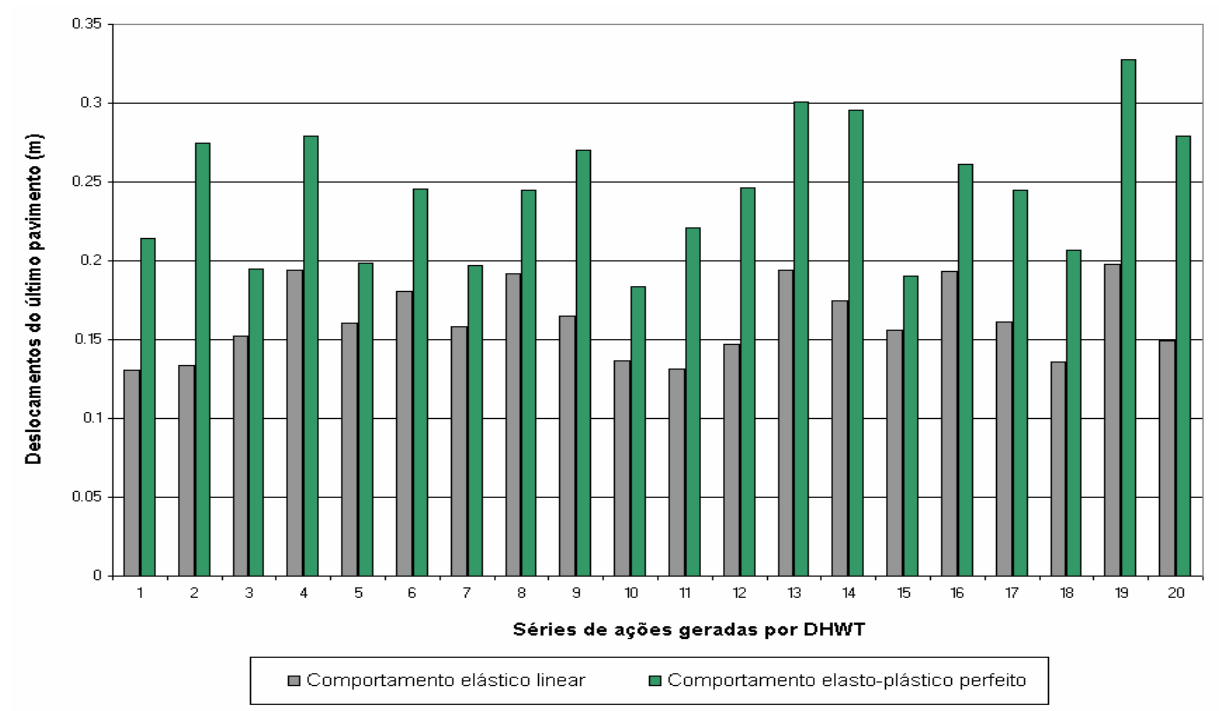

Figura 6.26. Deslocamentos do último pavimento devido a ações geradas por DHWT.

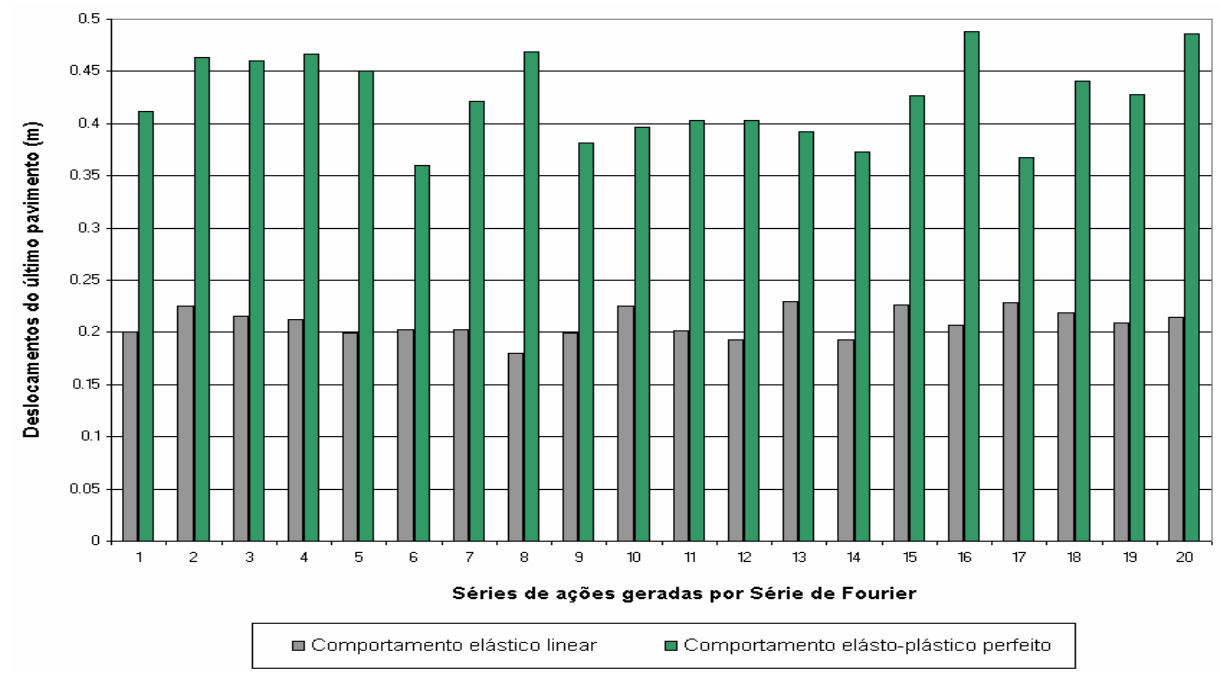

Figura 6.27. Deslocamentos do último pavimento devido a ações geradas por Série de Fourier.

São escolhidos então os deslocamentos de $u_{4}$ que melhor representam os valores médios das séries de cada modelo, para estes são analisados seus parâmetros estatísticos, como média e desvio padrão, e assim, são calculados seus erros relativos, em relação aos deslocamentos de $u_{4}$ ocasionados pelo sismo ocorrido em Hachinohe. 
Tabela 6.3. Dados estatísticos dos deslocamentos sofridos pela estrutura devido às séries dos dois modelos empregados, comparados ao deslocamento devido ao sismo real.

\begin{tabular}{|c|c|c|c|}
\hline \multicolumn{4}{|c|}{ Sismo em Hachinohe $(m)$} \\
\hline \multicolumn{2}{|c|}{ Comportamento elástico linear } & \multicolumn{2}{|c|}{ Comportamento elasto-plástico perfeito } \\
\hline Valor Máximo & 0.15824 & Valor Máximo & 0.17477 \\
\hline \multicolumn{4}{|c|}{ Séries de ações geradas a partir de DHWT (m) } \\
\hline \multicolumn{2}{|c|}{ Comportamento elástico linear } & \multicolumn{2}{|c|}{ Comportamento elasto-plástico perfeito } \\
\hline $\mathrm{E}[\mathrm{x}]$ & 0.1620 & $\mathrm{E}[\mathrm{x}]$ & 0.2437 \\
\hline$\sigma_{\mathrm{x}}$ & 0.02325 & $\sigma_{\mathrm{x}}$ & 0.04187 \\
\hline $\mathrm{E}[\mathrm{x}]+1.65 \sigma_{\mathrm{x}}$ & 0.2004 & $\mathrm{E}[\mathrm{x}]+1.65 \sigma_{\mathrm{x}}$ & 0.3128 \\
\hline $\operatorname{Erro}(\mathrm{E}[\mathrm{x}]) \%$ & 2.3761 & $\operatorname{Erro}(\mathrm{E}[\mathrm{x}]) \%$ & 39.4404 \\
\hline $\operatorname{Erro}\left(\mathrm{E}[\mathrm{x}]+1.65 \sigma_{\mathrm{x}}\right) \%$ & 26.6431 & $\operatorname{Erro}\left(E[x]+1.65 \sigma_{x}\right) \%$ & 78.9781 \\
\hline \multicolumn{4}{|c|}{ Séries de ações geradas a partir de Série de Fourier $(\mathrm{m})$} \\
\hline \multicolumn{2}{|c|}{ Comportamento elástico linear } & \multicolumn{2}{|c|}{ Comportamento elasto-plástico perfeito } \\
\hline $\mathrm{E}[\mathrm{x}]$ & 0.2092 & $\mathrm{E}[\mathrm{x}]$ & 0.4244 \\
\hline$\sigma_{\mathrm{x}}$ & 0.01374 & $\sigma_{\mathrm{x}}$ & 0.03986 \\
\hline $\mathrm{E}[\mathrm{x}]+1.65 \sigma_{\mathrm{x}}$ & 0.2319 & $\mathrm{E}[\mathrm{x}]+1.65 \sigma_{\mathrm{x}}$ & 0.4901 \\
\hline $\operatorname{Erro}(\mathrm{E}[\mathrm{x}]) \%$ & 32.2042 & $\operatorname{Erro}(\mathrm{E}[\mathrm{x}]) \%$ & 142.8334 \\
\hline $\operatorname{Erro}\left(E[x]+1.65 \sigma_{x}\right) \%$ & 46.5495 & $\operatorname{Erro}\left(\mathrm{E}[\mathrm{x}]+1.65 \sigma_{\mathrm{x}}\right) \%$ & 180.4257 \\
\hline
\end{tabular}

Como pode ser observado, para o modelo desenvolvido neste trabalho, a série de número 19 possui valores próximos dos valores característicos, e para o modelo gerado por Série de Fourier, a série de número 20 apresenta os valores mais próximos dos valores característicos. Estas respostam podem ser verificadas pelas Figuras 6.28, 6.29 e 6.30: 


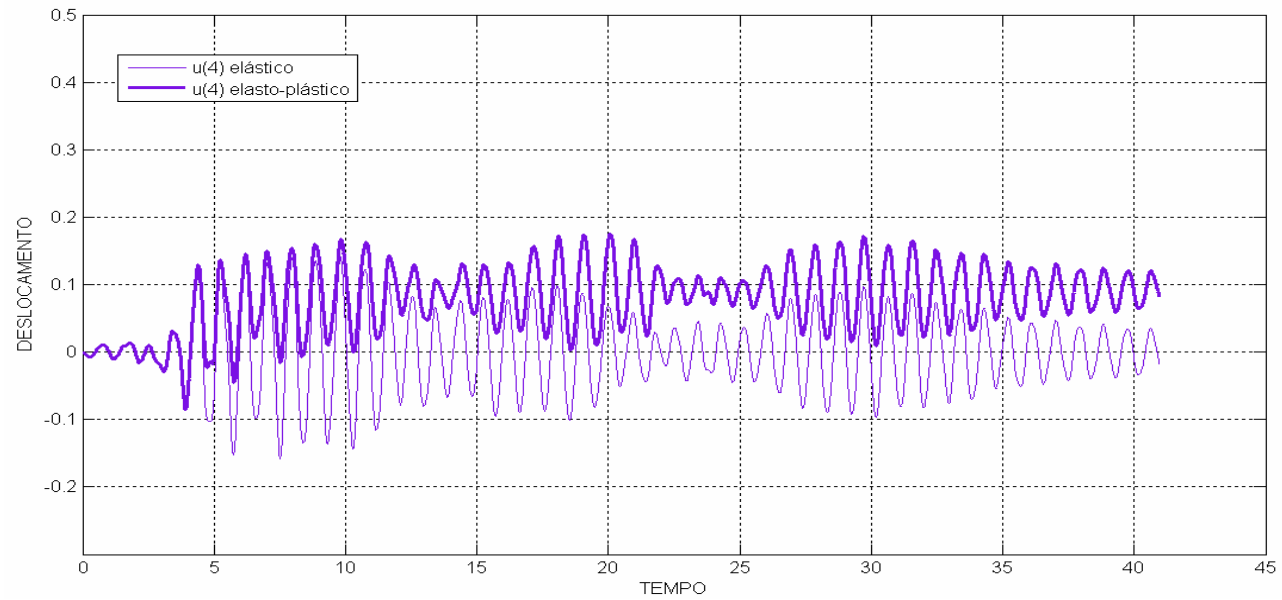

Figura 6.28. Resposta da estrutura devido ao acelerograma de Hachinohe.

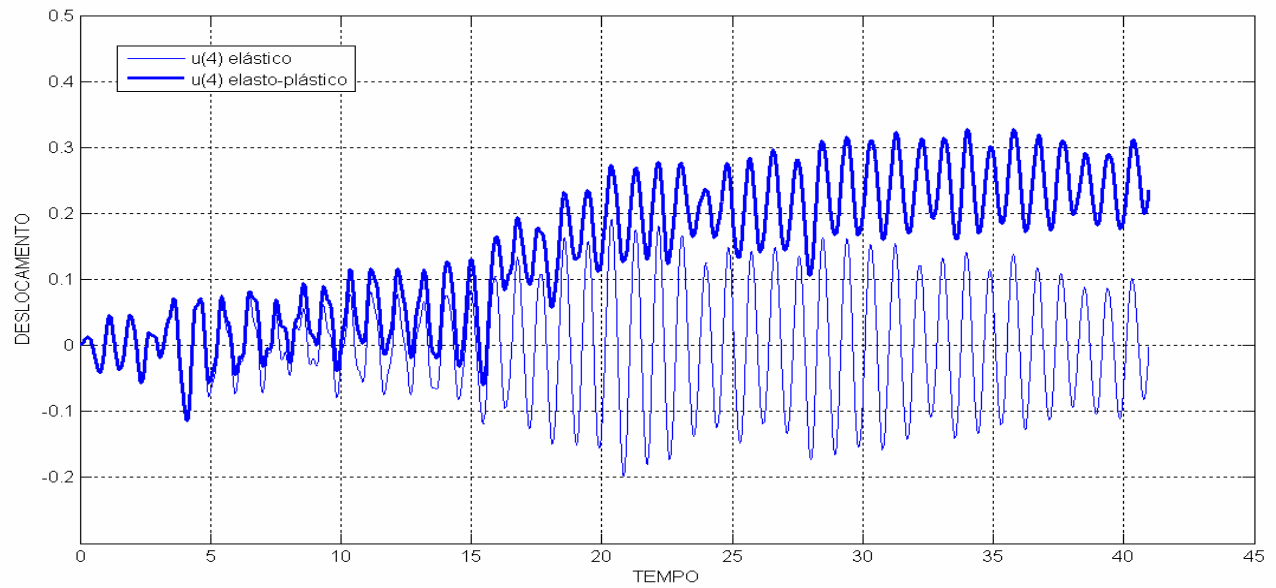

Figura 6.29. Resposta da estrutura devido à série 19, geradas a partir de DHWT.

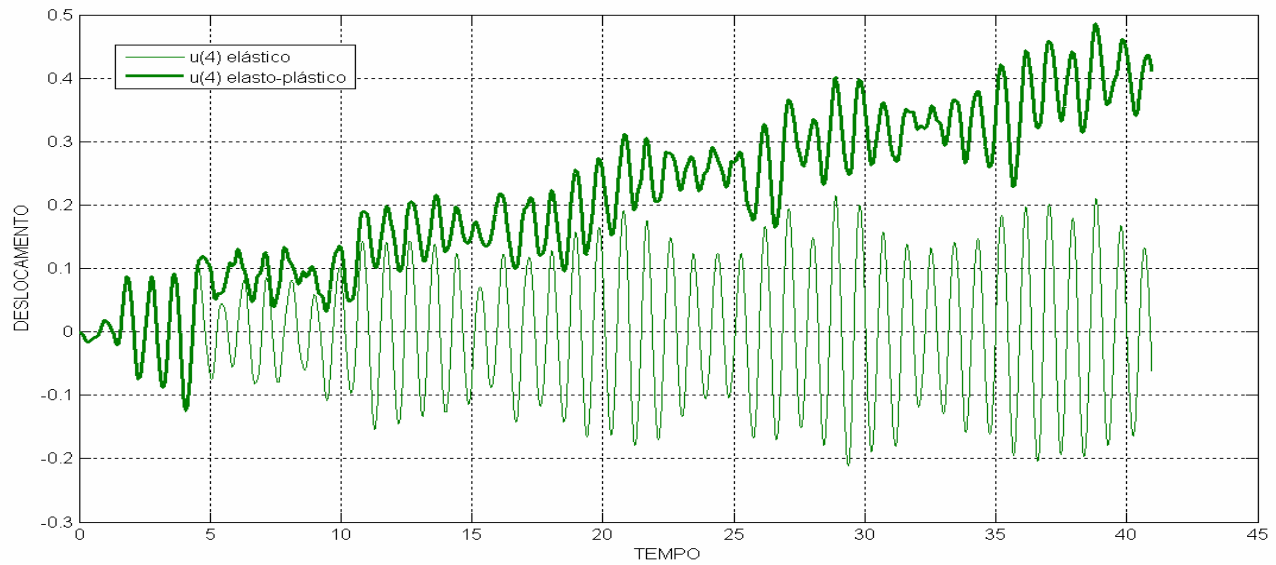

Figura 6.30. Resposta da estrutura devido à série 20, geradas a partir de SF.

Percebe-se neste caso, que para ações geradas por Série de Fourier, as respostas estruturais tendem a ser bem maiores que as respostas estruturais devido ao sismo real, e as respostas às ações geradas a partir de DHWT possuem valores intermediários, podendo ocasionar certa margem de segurança com economia no dimensionamento da estrutura. 


\subsection{EXEMPLO 4}

Neste exemplo e utilizada uma ação devido a sismo, baseado no sinal da componente horizontal da aceleração de um terremoto de magnitude 7.1 da escala Richter, ocorrido na cidade de El-Centro, em 18 de maio de 1940 na Califórnia. O modelo estrutural de shear building adotado possui 4 pavimentos, cada um com 4 pilares de aço e lajes de concreto.

Todos os dados são apresentados a seguir:

- Quantidade de pavimentos:

- Valor da aceleração máxima $\left(\mathrm{m} / \mathrm{s}^{2}\right)$ :

- Quantidade de pontos analizados:

- Valor de $\beta$ utilizado:

- Taxa de amortecimento do solo:

- Freqüência fundamental do solo ( $\mathrm{rad} / \mathrm{s})$ :

- Tensão de escoamento do aço (MPa):

- Módulo de elasticidade do aço (MPa):

- Tipos de perfis métalicos:

- Momento de Inércia dos pilares $\left(\mathrm{m}^{4}\right)$ :

- Módulo de resistência plástica $\left(\mathrm{m}^{3}\right)$ :

- Altura dos pilares por pavimento (m): $\mathrm{m}=4$

$\ddot{u}_{\text {max }}=3.495$

$\mathrm{N}=2752$

$\beta=1 / 6$

$\xi_{\mathrm{g}}=0.4$

$\omega_{\mathrm{g}}=12.56$

$\mathrm{f}_{\mathrm{y}}=250$

$\mathrm{E}=210000$

CS200x39 pavimentos 1 à 4

$\mathrm{I}_{1}=\mathrm{I}_{2}=\mathrm{I}_{3}=\mathrm{I}_{4}=1.267 \times 10^{-5}$

$Z_{1}=Z_{2}=Z_{1}=Z_{2}=1.92 \times 10^{-5}$

$\ell_{1}=\ell_{2}=\ell_{3}=\ell_{4}=3$

Podem-se obter as seguintes características estruturais:

- Massa das lajes (kg):

- Rigidez elástica dos pilares por pavimento $(\mathrm{N} / \mathrm{m})$ :

- Frequiências naturais da estrutura ( $\mathrm{rad} / \mathrm{s})$ :

- Taxas de amortecimento da estrutura:

- Cortantes plásticos dos pilares por pavimento $(\mathrm{N})$ : $m_{1}=m_{2}=25000$;

$m_{3}=m_{4}=15000$

$k_{1}=k_{2}=k_{3}=k_{4}=4730133$;

$\omega_{\mathrm{f}}=5.7129 ; \quad \omega_{\mathrm{f}_{2}}=15.0474$;

$\omega_{\mathrm{f}_{3}}=22.8777 ; \omega_{\mathrm{f}_{4}}=30.338$

$\xi_{1}=0.01 ; \xi_{2}=0.01$

$\mathrm{Qpl}_{1}=\mathrm{Qpl}_{2}=128000$;

$\mathrm{Qpl}_{3}=\mathrm{Qpl}_{4}=128000$ 
As características do sinal de referência utilizado são:

- Tempo do sinal original (s):

$\mathrm{T}=56$

- Tempo do sinal modificado:

$\mathrm{T}=55.02$

- Incremento de tempo (s):

$\Delta \mathrm{t}=0.02$

- Menor freqüência do sinal original (Hz):

0.0278

- Maior freqüência sem repetição do espectro (Hz): 25

- Maior freqüência do sinal original $(\mathrm{Hz})$ : 50

- Menor freqüência do sinal modificado $(\mathrm{Hz})$ :

- Maior freqüência sem repetição do espectro $(\mathrm{Hz})$ : 25

- Maior frequiência do sinal modificado $(\mathrm{Hz})$ :

As características do sinal gerado por HDWT são:

- Tempo do sinal gerado (s):

$\mathrm{T}=55.02$

- Incremento de tempo (s):

$\Delta \mathrm{t}=0.02$

- Variação da janela tempo-frequência:

Aritmética

- Fator de variação:

$\mathrm{p}=25$

- Quantidade de níveis:

$\mathrm{n}=11$

- Nível que possui a freqüência fundamental

jf $=2$

- Menor frequiência do sinal gerado ( $\mathrm{rad} / \mathrm{s})$ :

$\omega^{\mathrm{w}-}=0.1141$

- Maior frequiência sem repetição do espectro (rad/s):

$\omega^{\mathrm{w}+}{ }_{\mathrm{n}-1}=157.1770$

As características do sinal gerado por Série de Fourier são:

- Tempo do sinal gerado (s):

$\mathrm{T}=55.02$

- Incremento de tempo (s):

$\Delta \mathrm{t}=0.02$

- Variação do intervalo de frequência:

Aritmética

- Fator de variação:

$\mathrm{p}=0.1284$

- Quantidade de harmônicos:

$\mathrm{n}=80$

- Nível que possui a frequiência fundamental:

if $=15$

- Menor frequiência do sinal gerado ( $\mathrm{rad} / \mathrm{s})$ :

$\omega^{\mathrm{F}-}=0.3769$

- Maior freqüência sem repetição do espectro ( $\mathrm{rad} / \mathrm{s})$ :

$\omega_{\mathrm{n}}^{\mathrm{F}+}=157.1774$ 
A aceleração do solo atua na direção de menor inércia dos perfis metálicos, onde todos os perfis são considerados de massa desprezível. O espectro de frequência utilizado é o (5.97) para ambas as ações geradas, e a função de tendência, usada para a ação de comportamento não estacionário é uma composição entre um polinômio e uma função exponencial. Estes podem ser descritos, respectivamente:

$$
\begin{aligned}
& \frac{\mathrm{W}_{a c}(\omega)}{\sigma_{a c}{ }^{2}}=\frac{4 \xi_{\mathrm{g}}}{\omega_{\mathrm{f}} \pi\left(1+4 \xi_{\mathrm{g}}{ }^{2}\right)} \frac{\mathrm{r}_{\omega}\left(1+4 \xi_{\mathrm{g}}{ }^{2} \mathrm{r}_{\omega}{ }^{2}\right)}{\left(\left(1-\mathrm{r}_{\omega}{ }^{2}\right)^{2}+4 \xi_{\mathrm{g}}{ }^{2} \mathrm{r}_{\omega}{ }^{2}\right)} \quad \text { para } \quad \mathrm{r}_{\omega}=\left(\frac{\omega}{\omega_{\mathrm{g}}}\right) \\
& \mathrm{f}(\mathrm{t})=\left\{\begin{array}{lll}
400 \mathrm{t}^{2} & \text { para } & 0 \leq \mathrm{t}<0.1 \\
\mathrm{e}^{-3.1(\mathrm{t}-0.1)} & \text { para } & 0.1 \leq \mathrm{t}<1
\end{array}\right.
\end{aligned}
$$

Na figura 6.31(a) e 6.31(b), podem ser vistos (6.36) e (6.37):
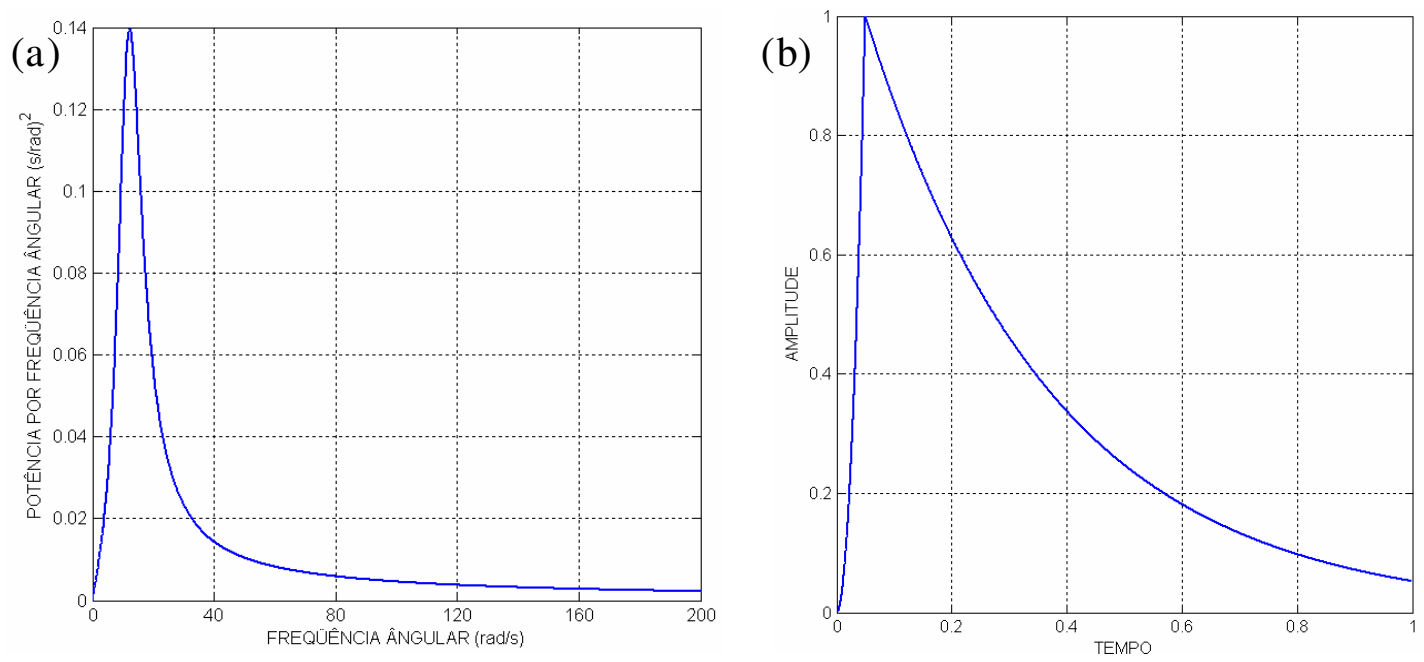

Figura 6.31. São as curvas: (a) Espectro de frequiência; (b) Função de tendência.

Em posse destes dados, os dois tipos de ações são geradas na forma normalizada e então multiplicadas por valores que possuam amplitudes máximas equivalentes a amplitude máxima da ação original, estas duas ações, juntamente com a ação original são mostradas nas Figuras 6.32, 6.33 e 6.34 .

Para a comparação dos três modelos, devido ao fato dos acelerogramas serem gerados com a ajuda de uma função pseudo-aleatória, são analisados os escalogramas, através do uso da transformada da wavelet harmônica; e do espectro de potência, através do uso da transformada de Fourier. Todas estas são oriundas dos acelerogramas, mostrados a seguir: 


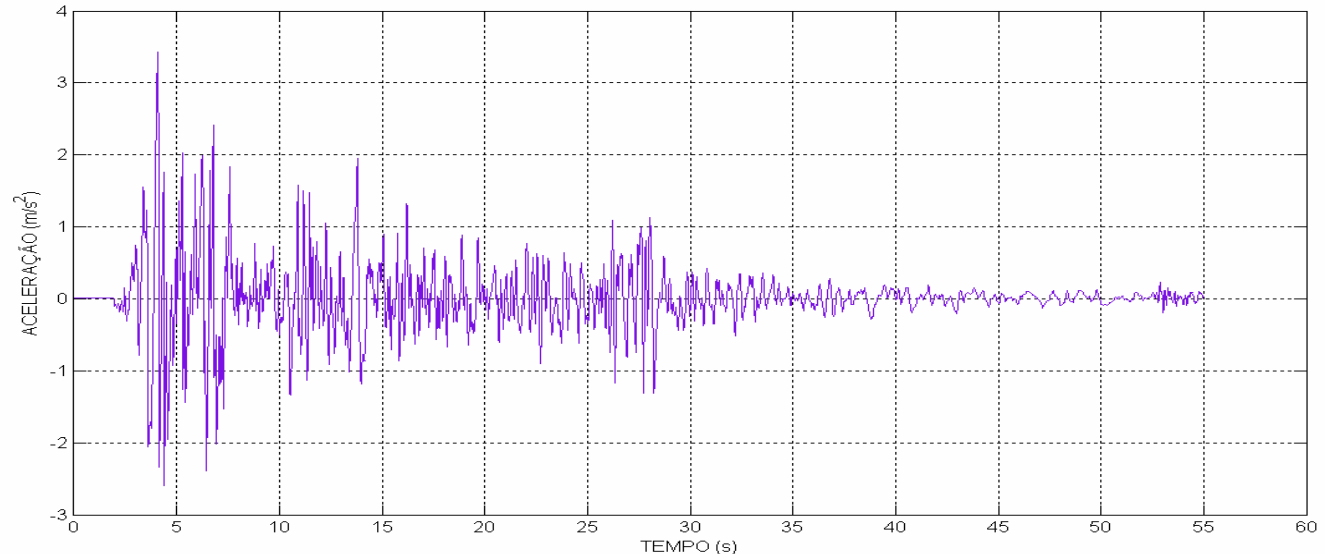

Figura 6.32. Acelerograma do terremoto ocorrido na cidade de El-centro, em 1940 na Califórnia.

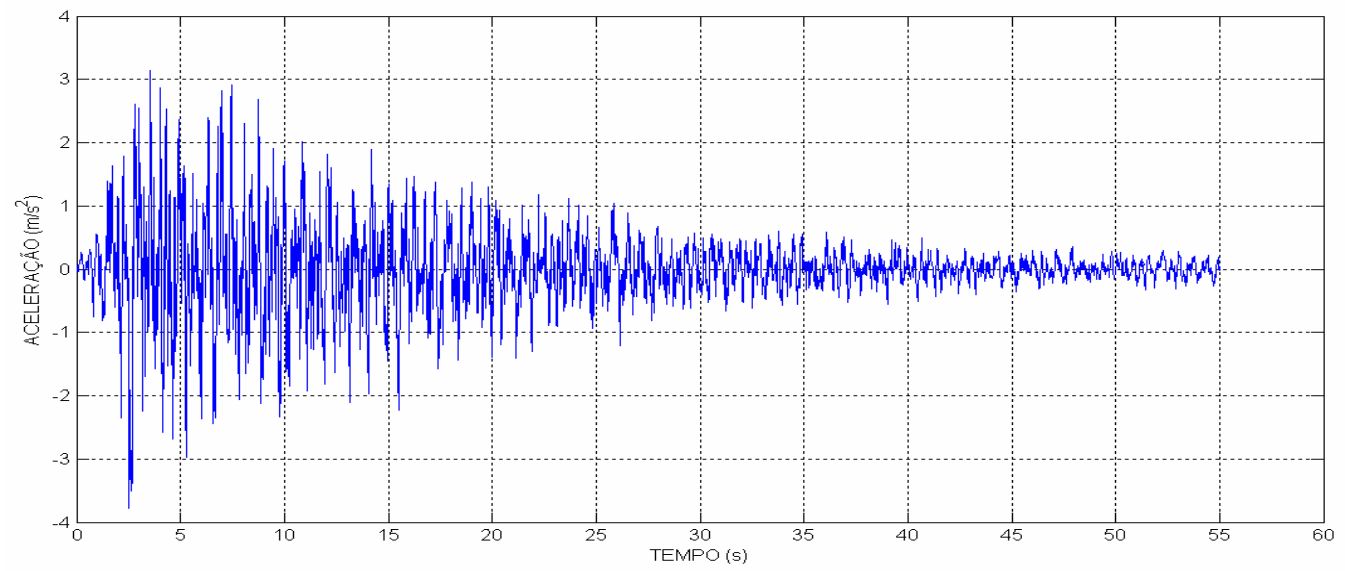

Figura 6.33. Acelerograma com comportamento não estacionário, gerado pelo a partir de DHWT.

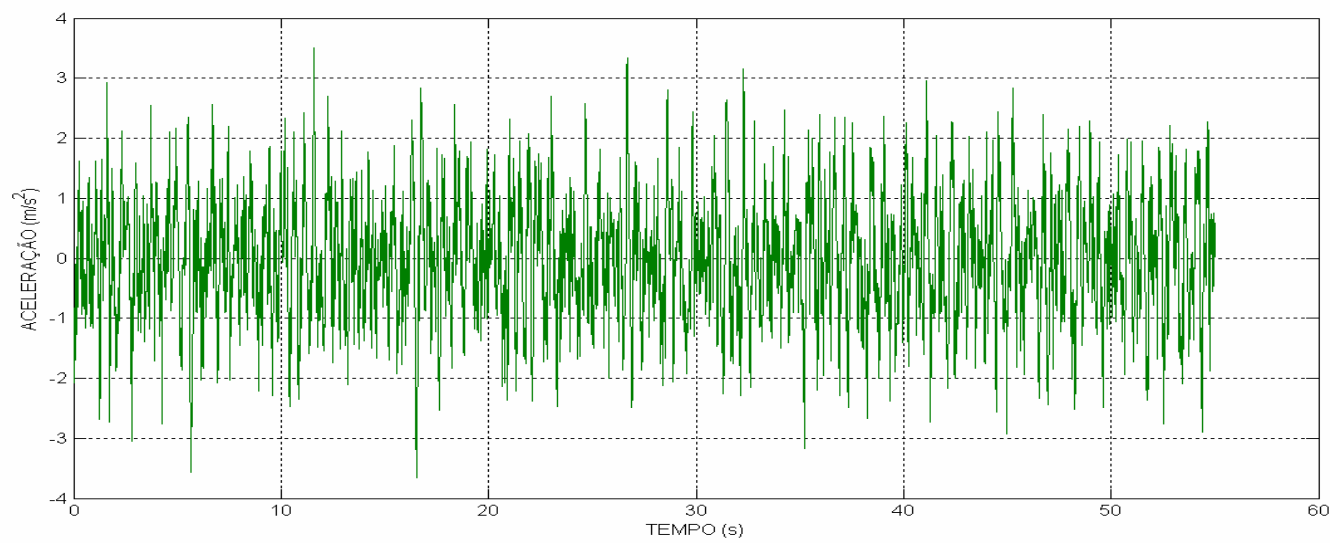

Figura 6.34. Acelerograma com comportamento estacionário, gerado a partir de Série de Fourier. 


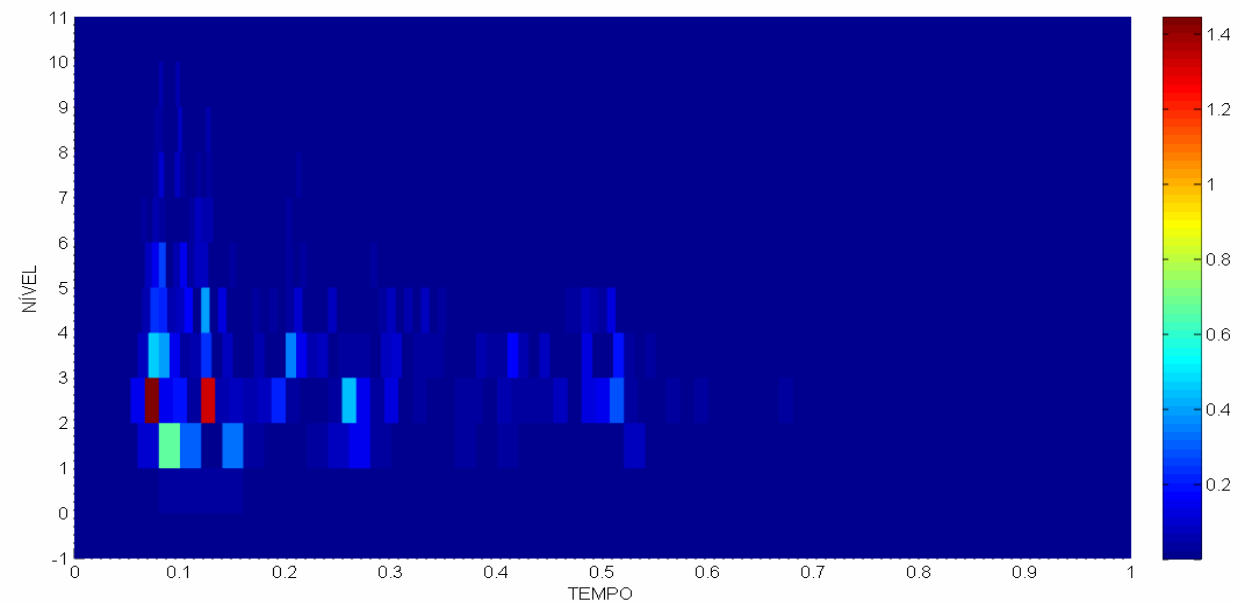

Figura 6.35. Escalograma do terremoto ocorrido na cidade de El-centro, em 1940 na Califórnia, baseado em seu acelerograma.

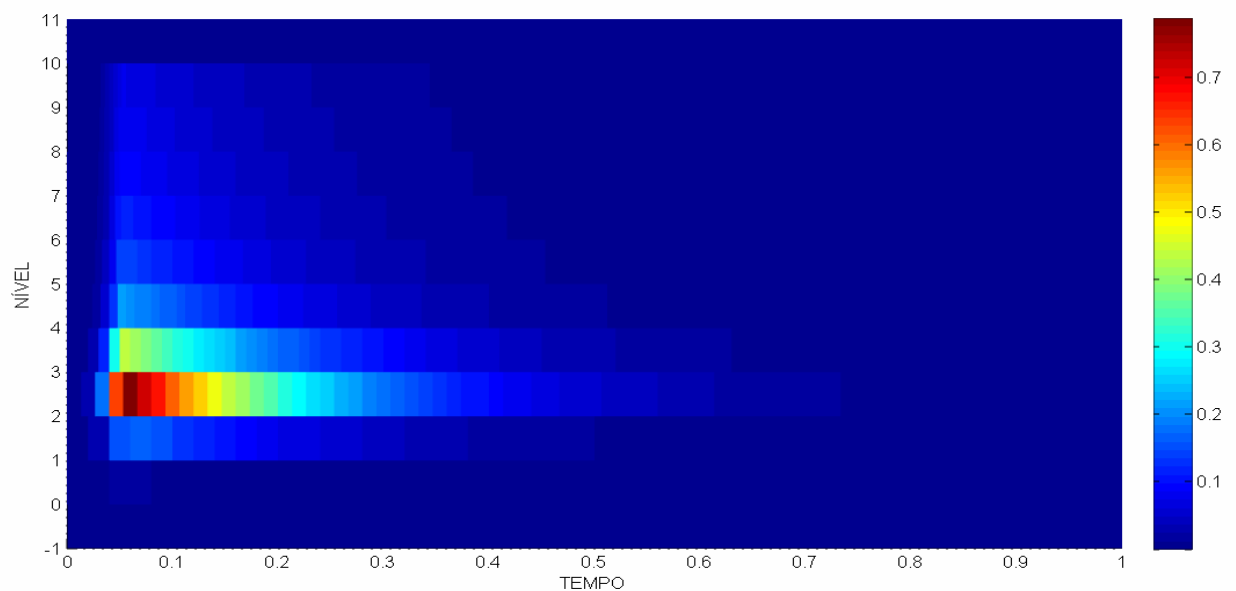

Figura 6.36. Escalograma do acelerograma com comportamento não estacionário, gerado a partir de DHWT.

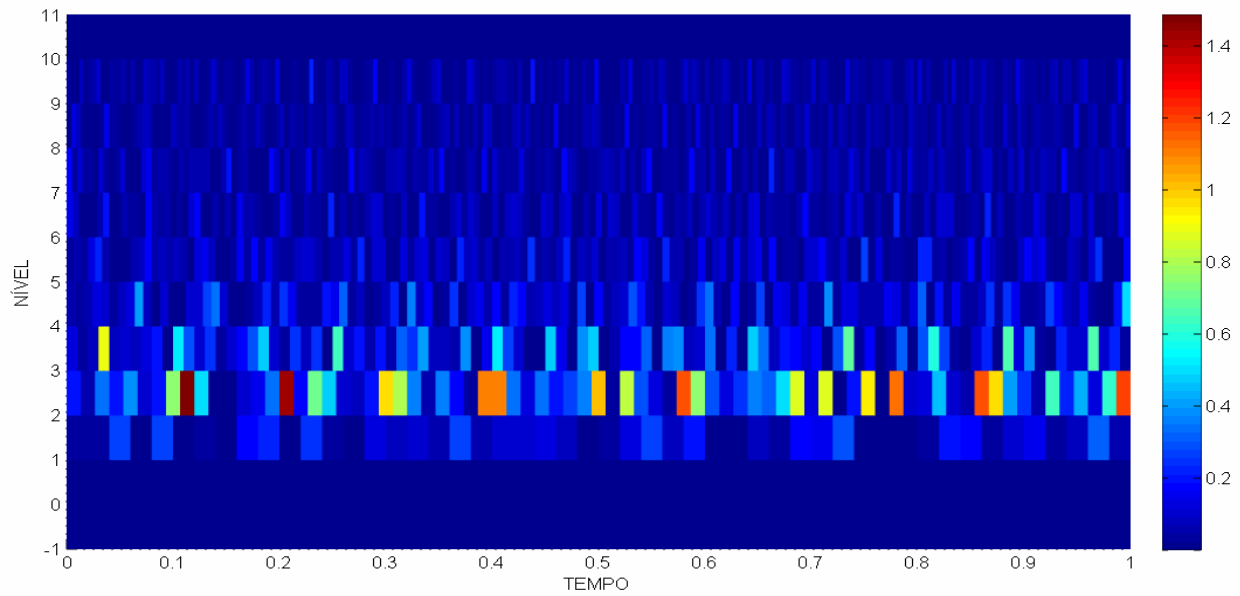

Figura 6.37. Escalograma do acelerograma com comportamento aproximadamente estacionário, gerado a partir de Série de Fourier. 


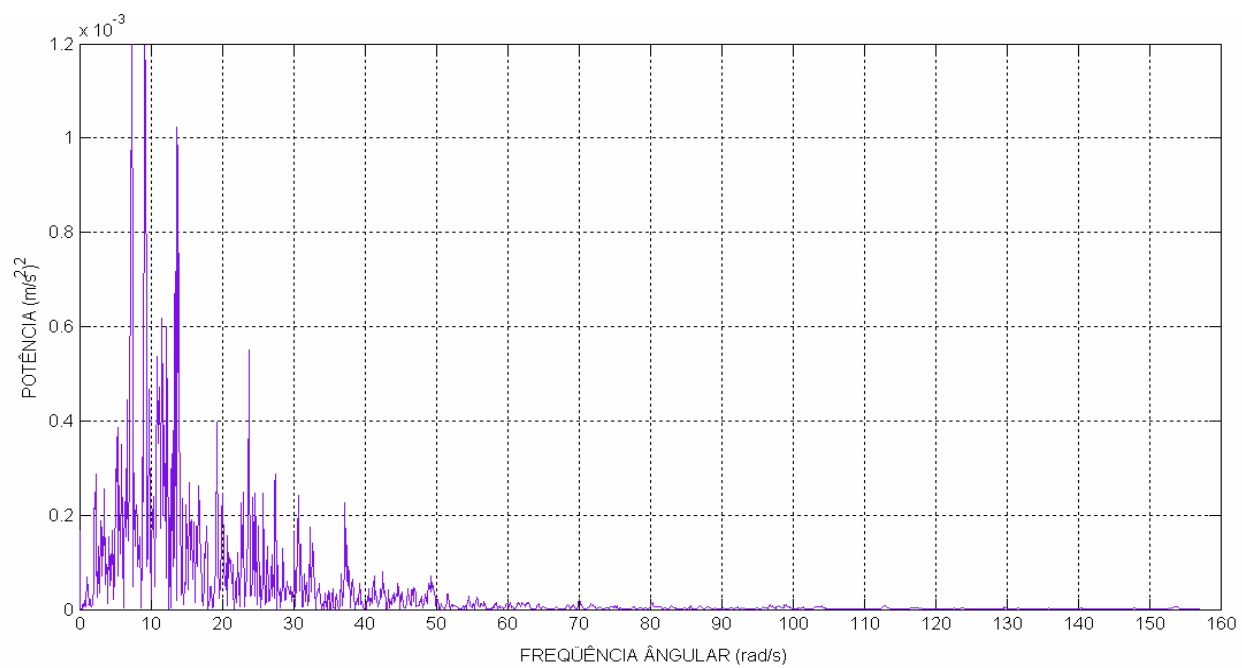

Figura 6.38. Espectro de potência do terremoto ocorrido na cidade de El-centro, em 1940 na Califórnia, baseado em seu acelerograma.

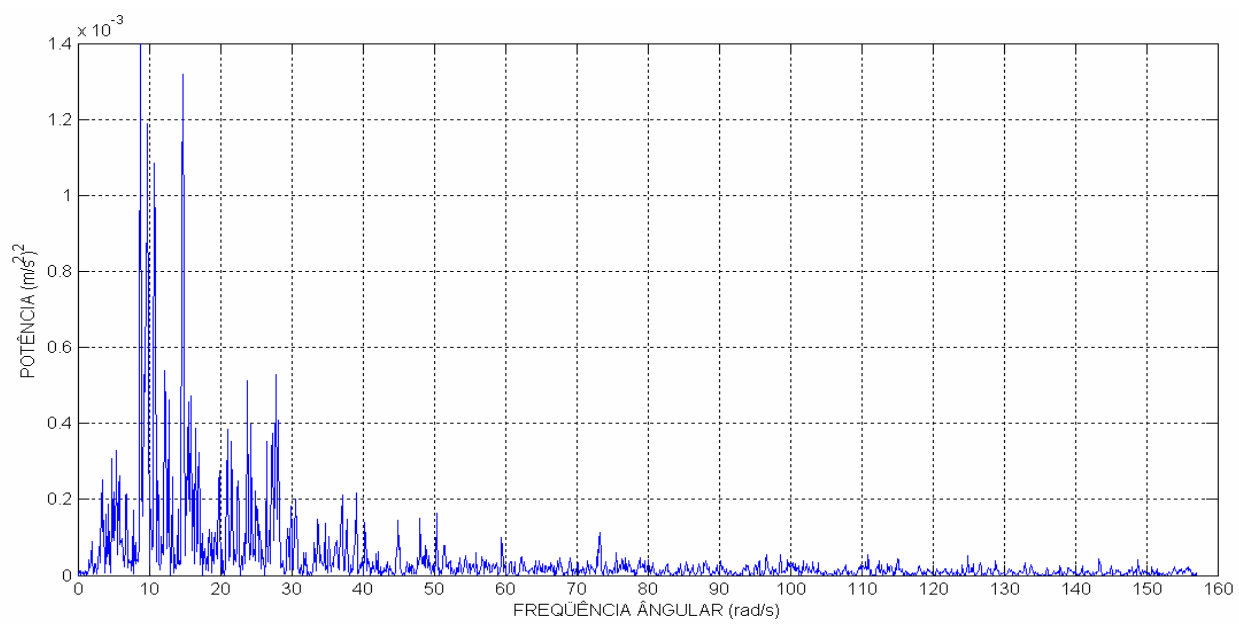

Figura 6.39. Espectro de potência do acelerograma com comportamento não estacionário, gerado a partir de DHWT.

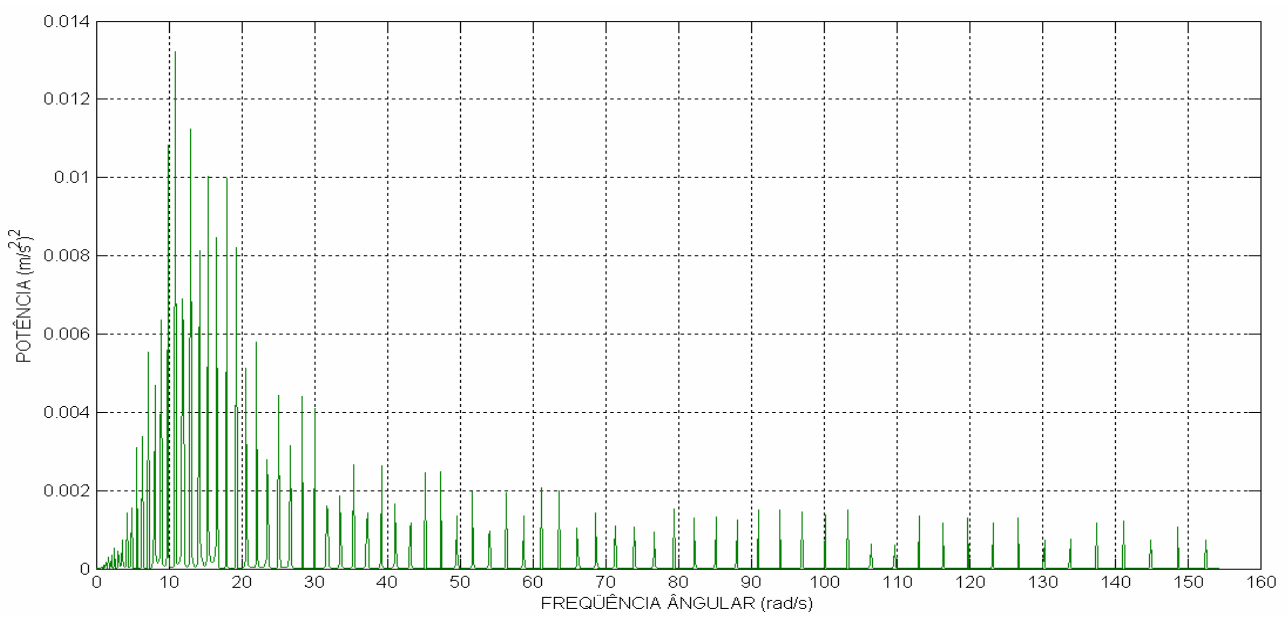

Figura 6.40. Espectro de potência do acelerograma com comportamento estacionário, gerado a partir de Série de Fourier. 
São então analisados os parâmetros estatísticos, como média e desvio padrão, e assim, são calculados seus erros relativos, em relação ao sismo ocorrido em El-centro.

Tabela 6.4. Comparação do Escalograma e do Espectro de potência dos dois métodos, com relação ao Escalograma e Espectro de Potência do sismo em El-centro.

\begin{tabular}{|c|c|c|c|c|c|c|}
\hline Sinal & $\mathrm{E}[\mathrm{x}]$ & $\begin{array}{c}\text { Erro(E[x]) } \\
\%\end{array}$ & $\sigma_{\mathrm{x}}$ & $\begin{array}{c}\text { Erro }\left(\sigma_{\mathrm{x}}\right) \\
\%\end{array}$ & $\mathrm{E}[\mathrm{x}] \mathrm{E}[\mathrm{y}]$ & $\sigma_{\mathrm{xy}}$ \\
\hline \multicolumn{7}{|c|}{ ESCALOGRAMA } \\
\hline El-centro & $1.6731 \mathrm{E}-2$ & & $7.4815 \mathrm{E}-2$ & & & \\
\hline DHWT & $1.8217 \mathrm{E}-2$ & 8.8879 & $1.1212 \mathrm{E}-1$ & 49.8629 & $3.7449 \mathrm{E}-3$ & 0.4100 \\
\hline SF & $2.1646 \mathrm{E}-2$ & 29.3766 & $1.3238 \mathrm{E}-1$ & 76.9390 & $1.9141 \mathrm{E}-3$ & 0.1567 \\
\hline \multicolumn{7}{|c|}{ ESPECTRO DE POTÊNCIA } \\
\hline El-centro & $3.6407 \mathrm{E}-5$ & & $1.0494 \mathrm{E}-4$ & & & \\
\hline DHWT & $4.9240 \mathrm{E}-5$ & 35.2804 & $1.5408 \mathrm{E}-3$ & 46.8221 & $7.4231 \mathrm{E}-8$ & 0.448 \\
\hline SF & $5.4377 \mathrm{E}-5$ & 49.3616 & $1.2632 \mathrm{E}-3$ & 14.8044 & $3.7625 \mathrm{E}-8$ & 0.2689 \\
\hline
\end{tabular}

Como pode ser observado, nas análises de covariância, o erro é quase duas vezes menor se comparado ao método que usa Série de Fourier, mostrando que embora a geração de ações com o uso de HDWT seja mais complexa, esta possui um bom potencial para gerar carregamentos mais próximos da realidade.

Agora, aplicando-se estas ações segundo o processo descrito anteriormente e realizando a análise dinâmica incremental pelo método de Newmark, podemos obter a resposta dinâmica da estrutura para vinte séries de ações de cada modelo.

É apresentada na Figura 6.41, as respostas estruturais dos valores extremos dos deslocamentos de $u_{4}$, para um comportamento elástico linear e elasto-plástico perfeito com ações geradas a partir de DHWT, e na Figura 6.42, para um comportamento elástico linear e elasto-plástico perfeito com ações geradas a partir de Série de Fourier: 


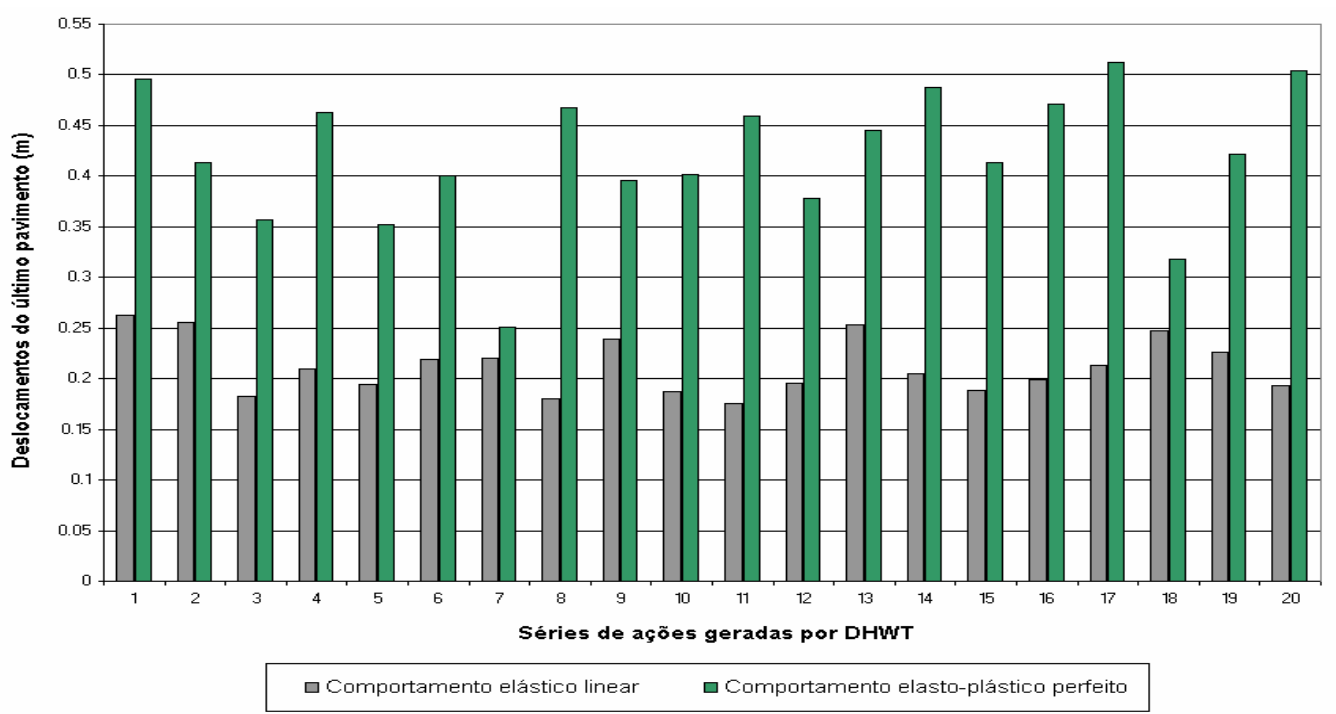

Figura 6.41. Deslocamentos do último pavimento devido a ações geradas por DHWT.

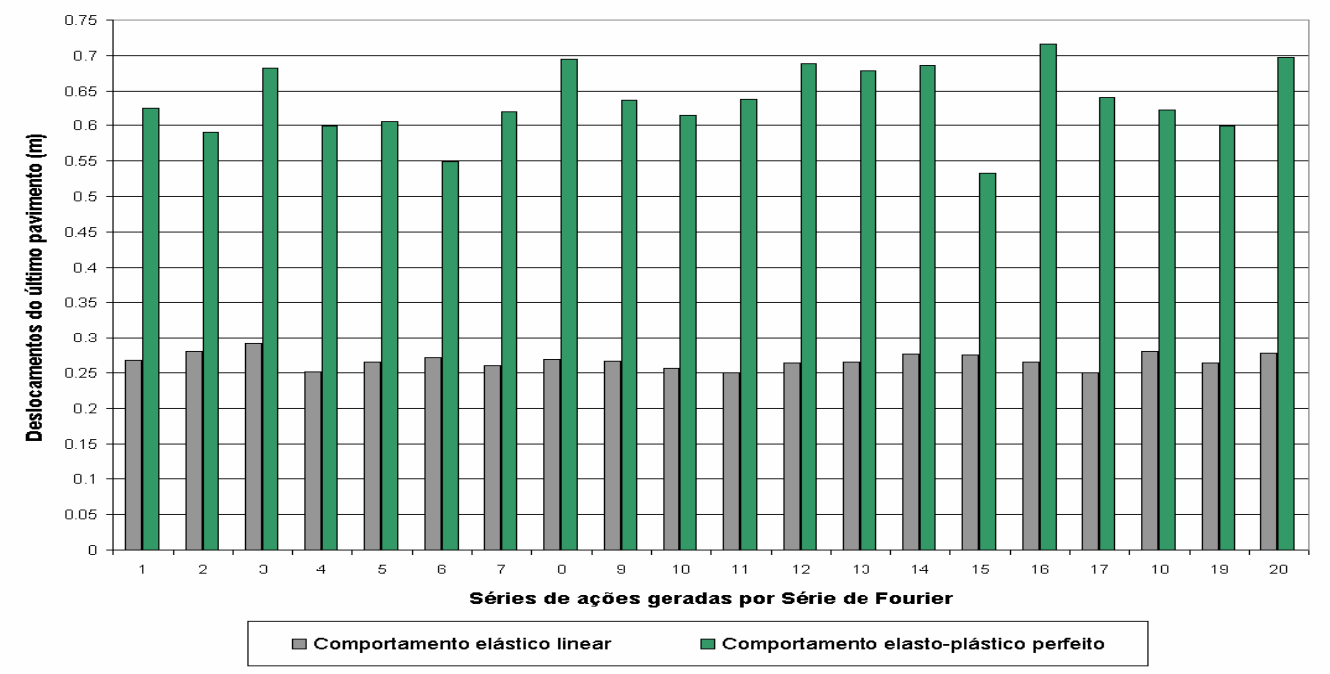

Figura 6.42. Deslocamentos do último pavimento devido a ações geradas por Série de Fourier.

São escolhidos então os deslocamentos de $u_{4}$ que melhor representam os valores médios das séries de cada modelo, para estes são analisados seus parâmetros estatísticos, como média e desvio padrão, e assim, são calculados seus erros relativos, em relação aos deslocamentos de $u_{4}$ ocasionados pelo sismo ocorrido em El-centro. 
Tabela 6.5. Dados estatísticos dos deslocamentos sofridos pela estrutura devido às séries dos dois modelos empregados, comparados ao deslocamento devido ao sismo real.

\begin{tabular}{|c|c|c|c|}
\hline \multicolumn{4}{|c|}{ Sismo em El-centro } \\
\hline \multicolumn{2}{|c|}{ Comportamento elástico linear } & \multicolumn{2}{|c|}{ Comportamento elasto-plástico perfeito } \\
\hline Valor Máximo & 0.21185 & Valor Máximo & 0.32398 \\
\hline \multicolumn{4}{|c|}{ Séries de ações geradas a partir de DHWT (m) } \\
\hline \multicolumn{2}{|c|}{ Comportamento elástico linear } & \multicolumn{2}{|c|}{ Comportamento elasto-plástico perfeito } \\
\hline $\mathrm{E}[\mathrm{x}]$ & 0.2123 & $\mathrm{E}[\mathrm{x}]$ & 0.4203 \\
\hline$\sigma_{\mathrm{x}}$ & 0.02721 & $\sigma_{\mathrm{x}}$ & 0.06712 \\
\hline $\mathrm{E}[\mathrm{x}]+1.65 \sigma_{\mathrm{x}}$ & 0.2572 & $\mathrm{E}[\mathrm{x}]+1.65 \sigma_{\mathrm{x}}$ & 0.5311 \\
\hline $\operatorname{Erro}(\mathrm{E}[\mathrm{x}]) \%$ & 0.2181 & $\operatorname{Erro}(\mathrm{E}[\mathrm{x}]) \%$ & 29.7452 \\
\hline $\operatorname{Erro}\left(E[x]+1.65 \sigma_{x}\right) \%$ & 21.4111 & $\operatorname{Erro}\left(E[x]+1.65 \sigma_{x}\right) \%$ & 63.9277 \\
\hline \multicolumn{4}{|c|}{ Séries de ações geradas a partir de Série de Fourier $(\mathrm{m})$} \\
\hline \multicolumn{2}{|c|}{ Comportamento elástico linear } & \multicolumn{2}{|c|}{ Comportamento elasto-plástico perfeito } \\
\hline $\mathrm{E}[\mathrm{x}]$ & 0.2674 & $\mathrm{E}[\mathrm{x}]$ & 0.6357 \\
\hline$\sigma_{\mathrm{x}}$ & 0.01090 & $\sigma_{\mathrm{x}}$ & 0.05021 \\
\hline $\mathrm{E}[\mathrm{x}]+1.65 \sigma_{\mathrm{x}}$ & 0.2854 & $\mathrm{E}[\mathrm{x}]+1.65 \sigma_{\mathrm{x}}$ & 0.7186 \\
\hline $\operatorname{Erro}(\mathrm{E}[\mathrm{x}]) \%$ & 26.2252 & $\operatorname{Erro}(\mathrm{E}[\mathrm{x}]) \%$ & 96.2177 \\
\hline $\operatorname{Erro}\left(\mathrm{E}[\mathrm{x}]+1.65 \sigma_{\mathrm{x}}\right) \%$ & 34.7167 & $\operatorname{Erro}\left(\mathrm{E}[\mathrm{x}]+1.65 \sigma_{\mathrm{x}}\right) \%$ & 121.7889 \\
\hline
\end{tabular}

Como pode ser observado, para o modelo desenvolvido neste trabalho, a série de número 1 possui valores próximos dos valores característicos, e para o modelo gerado por Série de Fourier, a série de número 20 apresenta os valores mais próximos dos valores característicos. Estas respostam podem ser verificadas pelas Figuras 6.43, 6.44 e 6.45: 


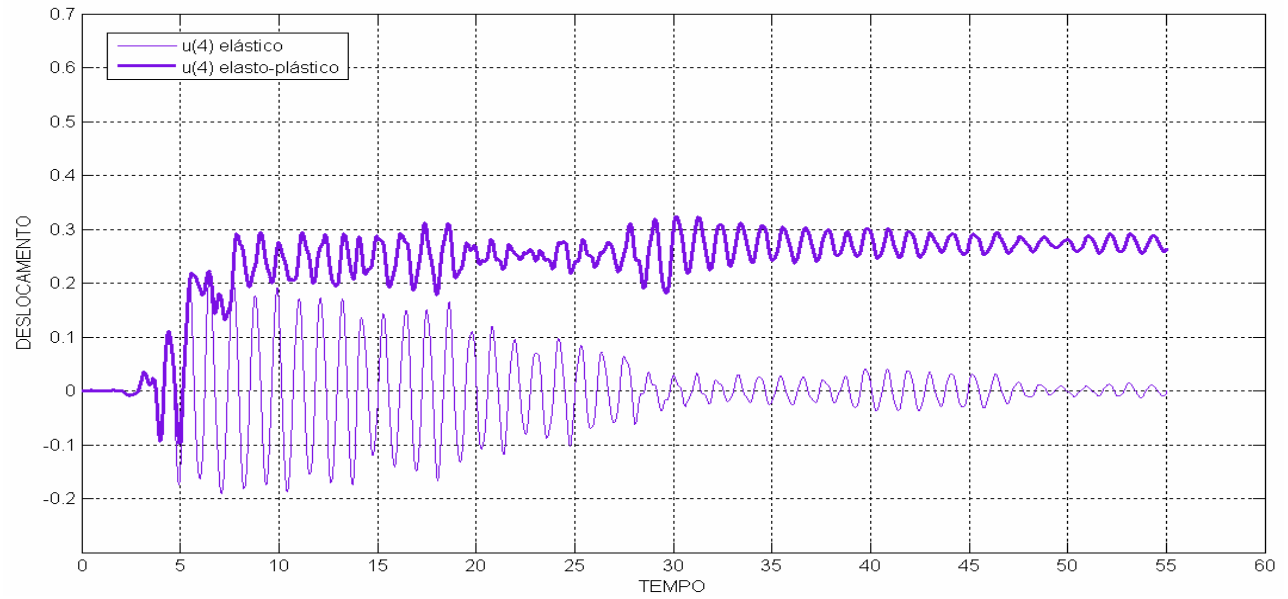

Figura 6.43. Resposta da estrutura devido ao acelerograma de El-Centro.

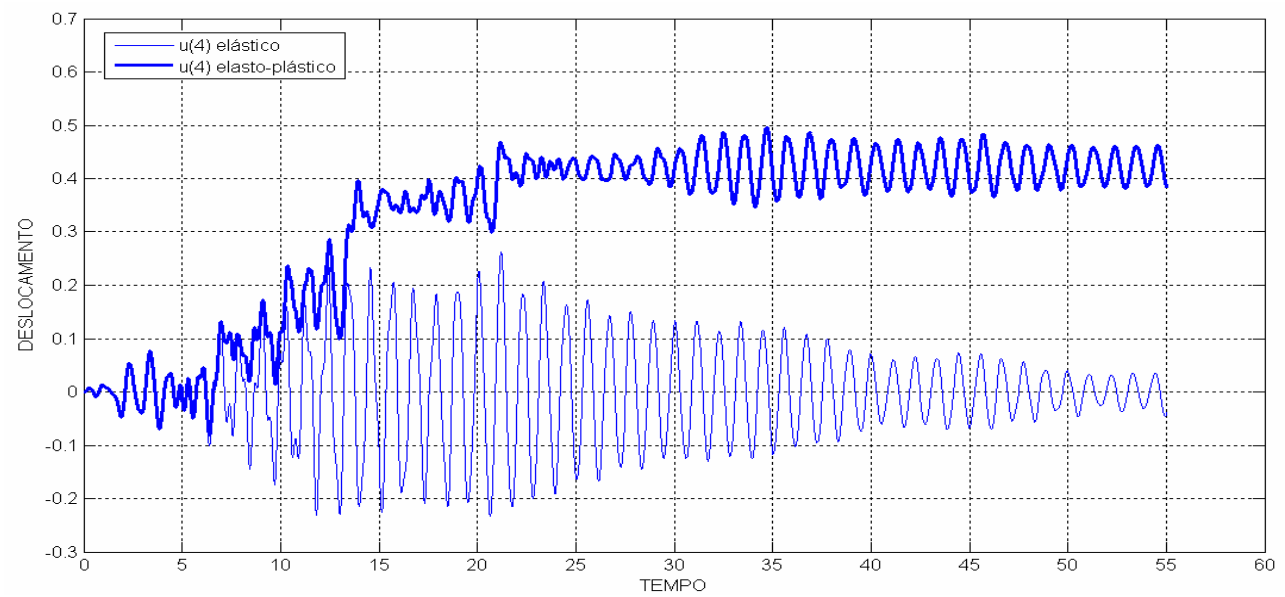

Figura 6.44. Resposta da estrutura devido à série 1, gerada a partir de DHWT.

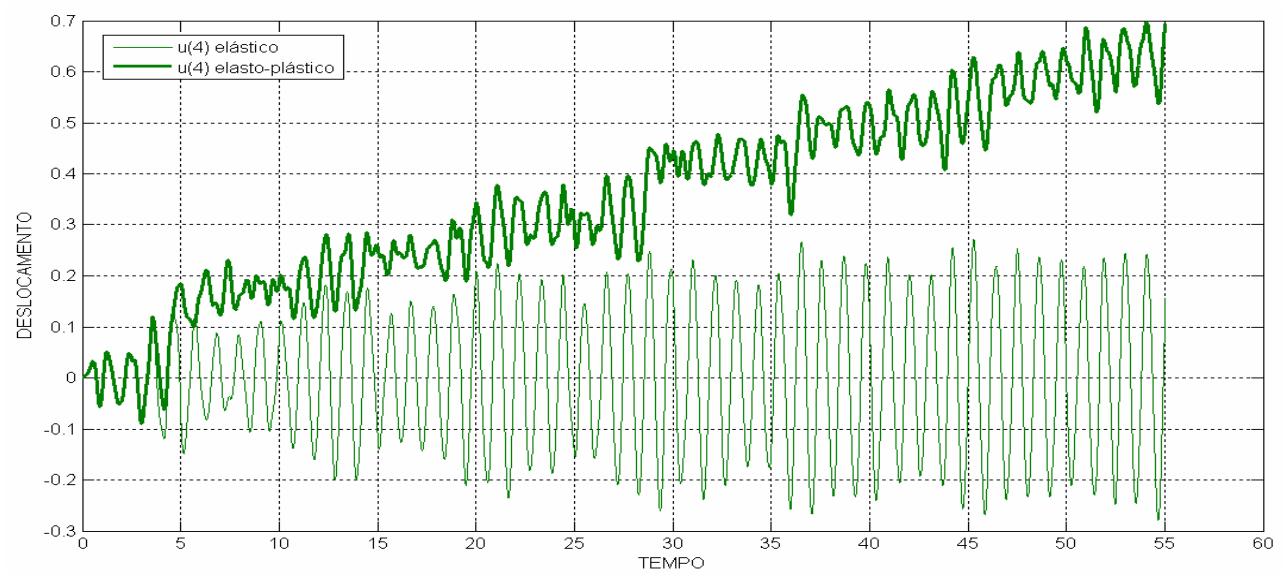

Figura 6.45. Resposta da estrutura devido à série 20, gerada a partir de SF.

Percebe-se igualmente ao exemplo anterior, que para ações geradas por Série de Fourier, as respostas estruturais tendem a ser bem maiores que as respostas estruturais devido ao sismo real, e as respostas às ações geradas a partir de DHWT possuem valores intermediários, podendo ocasionar certa margem de segurança com economia no dimensionamento da estrutura. 


\section{CONSIDERAÇÕES FINAIS}

\subsection{CONCLUSÃO}

Este trabalho teve o intuito de fundamentar a metodologia da geração de ações de comportamento não estacionário com o uso da transformada da wavelet harmônica, assim como a aplicação deste tipo de carregamento na estrutura, principalmente no que diz respeito a sismos e a correlação espacial das pressões do vento.

Embora este trabalho tenha mostrado bons resultados, pela geração de ações mais coerentes com a realidade, gerando deslocamentos mais próximos dos reais, é necessário lembrar que o estudo de geração de carregamentos com comportamento não estacionário para estruturas, embora viável teoricamente, e com grande potencial de utilização, até então foi pouco comparado com dados obtidos experimentalmente, ou em estruturas com outros tipos de comportamento de materiais além do elástico linear e elasto-plastico.

Embora seja muito provável quando se aplica uma ação não estacionária que, os deslocamentos sejam menores, pois as amplitudes extremas se concentram em um intervalo de tempo menor, esta diferença pode ser tão pequena em alguns casos, que não seja necessário um modelo mais complexo como este, pois o método de vento ou sismo sintético que possuem comportamento próximo do estacionário por utilizarem séries de Fourier se mostram com melhoria de precisão, embora que ainda inferior, quando este possui uma quantidade de harmônicos acima de 60 .

Na opnião deste autor, a maior vantagem de se utilizar métodos de geração de ações com comportamento não estacionário, é o controle, embora relativo, da relação entre o tempo e a frequiência, pois pode-se utilizar um escalograma com taxas de variações escolhidas para a janela tempo-freqüência, onde beneficie mais a freqüência ou o tempo dependendo da necessidade de sua análise, isto torna este tipo de geração de ações tão amplo que esta pode até simular um comportamento localmente estacionário, como mostrado em um dos exemplos do capítulo anterior. 


\subsection{SUGESTÕES PARA TRABALHOS FUTUROS}

O método de geração de ações que utiliza trasnformadas discretas de wavelets harmônicas pode e deve estar sujeito a ajustes, principalmente quanto à ponderação da frequiência de ressonância, pois a energia se distribui em uma faixa extensa de freqüências, diferentemente do modelo estacionário, a qual se concentra em poucas freqüências, podendo ser então desnecessária a consideração mostrada neste modelo que coloca a freqüência de ressonância no meio de um determinado nível.

Para trabalhos futuros, o uso de outros tipos de wavelets para a geração de carregamentos aleatórios, pode ser bastante interessante, desde que se consiga definir o elemento de variação pseudo-aleatória, que no caso das harmônicas, e das demais wavelets complexas, está na relação entre as parcelas reais e imaginárias das funções que as define.

Outras modificões na variação da janela tempo-freqüência também podem ser aplicadas, assim como o uso de várias funções de tendência para diferentes intervalos de frequiência, as quais seriam obtidas através de análises estatísticas de carregamentos filtrados para estas faixas de freqüência, gerando ações ainda mais próximas da realidade, e como citadas anteriormente, podem também ser estudadas as respostas destes carregamentos sintéticos em outros materiais ou modelos estruturais.

No geral, para trabalhos futuros, um melhor controle das janelas tempo-freqüência e análises estatísticas de espectros de potência no domínio da frequiência e do tempo seriam úteis para um maior desenvolvimento desta linha de pesquisa. 


\section{REFERÊNCIAS BIBLIOGRÁFICAS}

Aly, M. A., Vibration Control in Structures Due to Earthquake Effect Using MR

Damper. Alexandria: Tese (Doutorado) - Depatarment of Mechanical Enginenring, Alexandria University, 2005. 96p;

Barbosa, A.C.B.; Blitzkow, D. Ondeletas: Histórico e aplicações. São Paulo: Instituto de Astronomia, Geofísica e Ciências Atmosféricas da Universidade de São Paulo; Escola Politécnica da Universidade de São Paulo, 2008. 39p.

BLESSMANN, J. Introdução ao estudo das ações dinâmicas do vento. Porto Alegre: Editora da UFRGS, 1998. 282p.

BRASIL R.M.L.R.F. Dinâmica de Estruturas. São Paulo: Curso de Atualização, Departamento de Estruturas e Fundações, Escola Politécnica, Universidade de São Paulo, 2003. 94p.

BUCHHOLDT, H. A. An introduction to cable roof structures. London: 2 ed. Thomas Telford, 1999. 285p.

CAMPANHA, J.R. Sistemas Complexos e Aplicações. Rio Claro: Tese (Livre Docência)-Departamento de física, Instituto de Geociências e Ciências Exatas, Universidade Estadual Paulista, 2004.102p.

CARRIL JÚNIOR, C.F. Análise numérica e experimental do efeito do vento em torres metálicas treliçadas para telecomunicações. São Paulo: Tese (Doutorado) Escola Politécnica, Universidade de São Paulo, 2000. 143p.

CHAPMAN, S.J. Programação em MATLAB para engenheiros. São Paulo: Pioneira Thomson Learning, 2003. 477p.

CHIANN, C. Análise de ondeletas em séries temporais. São Paulo:Tese (Doutorado)Instituto de Matemática e Estatística, Universidade de São Paulo, 1997. 117p. 
CHIERICE, R.A.F. O uso da análise de Fourier, de wavelets e dos expoentes de lyapunov no estudo de um sistema dinâmico não-ideal com atrito seco e excitação externa. Rio Claro: Dissertação (Mestrado)-Instituto de Geociências e Ciências Exatas, Universidade Estadual Paulista, 2007. 92p.

CLOUGH,R.;PENZIEN, J. Dynamic of structures. New York: McGraw-Hill, 1975. $634 \mathrm{p}$.

CORBANI, S. Análise Dinâmica Elasto-Plástica de Estruturas Metálicas Sujeitas a Excitação Aleatória de Sismos. São Paulo: Dissertação (Mestrado), Departamento de Engenharia de Estruturas e Fundações, Escola Politécnica, Universidade de São Paulo, 2006. 64p.

FRANCO, M. Direct along-wind dynamic analysis of tall structures. São Paulo: Epusp, Boletim Técnico da Escola Politécnica da USP. Departamento de Engenharia de Estruturas e Fundações, BT/PEF/9303. 1993. 22p

Liu B. Adaptive harmonic wavelet transform with applications in vibration analysis. Singapore: Temasek Laboratories and Center of Wavelets, National University of Singapure, 2001. 64p

GOULD,P.L.;ABU-SITTA, S.H. Dynamic response of structures to wind and earth quakes loading. London: Pentech Press, 1980. 175p.

LAZANHA, E. C. Análise dinâmica elasto-plastica de estruturas metálicas sob excitação aleatória de vento. São Paulo, Dissertação (Mestrado) - Escola Politécnica, Universidade de São Paulo, 2003.142p

M. J. A. BOLZAN. Transformada em Ondeleta: Uma necessidade, Revista Brasileira de Ensino de Física, v. 28, n. 4, 2006. p. 563 - 567. 
MORETtin, P.A. Ondas e Ondeletas - da Análise de Fourier à Análise de

Ondeletas. São Paulo: Vol. 1, EDUSP, Instituto de Matemática e Estatística, Universidade de São Paulo, 1999. 267p.

NEWLAND, D.E. Harmônic Wavelet Analysis. Cambridge: Department of Engineering, University of Cambridge, 1993. p. 203-225.

NEWLAND, D.E. Random Vibrations, Spectral e Wavelet Analysis. Cambridge: Computer \& Structures, Vol. 1, Longman Publishers, 1993.478p.

NEWLAND, D.E. Harmônic and musical wavelets. Cambridge: Department of Engineering, University of Cambridge, 1994. p. 605-620.

NIGRO P.S.B. E BRASIL R.M.L.R.F. Geração de Carregamento Dinâmico de Estruturas Baseado em Transformada de Wavelet Harmônica, Departamento de Estruturas e Fundações, Escola Politécnica, Universidade de São Paulo, 2008. 16p.

PAZ,M.;LEIGH,W. Structural Dynamics Theory e Computation.Louisville;Orlando: speed Scientific School, University of Louisville; University of Central Florida, 5ed. Springer, 2004. 820p.

SPANOS; TEZCAN E TRATSKAS. Stochastic Processes Evolutionary Spectrum Estimation via Harmonic Wavelets, Comput. Methods Appl. Mech. Eng. Vol. 194, No. 1, 2005. pp. 1367-1383.

TEDESCO J. W.; MCDOUGAL W.G. E ROSS C.A. Structural Dynamics Theory and Aplication, Menlo Park, California, Addison Wesley Longman, Inc, Auburn University, 1999,816p

KRÉE, P.;SOIZE, C. Mathematics of random phenomena-random vibrations of mechanical structures. Dordrecht: Reidel Publishing Company, 1986. 438p.

WALKER, JAMES. S. Fast Fourier Transforms. Boca Raton: 2ed. CRC Press, 1996. $447 \mathrm{p}$. 